\title{
Prediction of life -threatening events in infants using heart rate variability measurements
}

Xueyan Xu

West Virginia University

Follow this and additional works at: https://researchrepository.wvu.edu/etd

\section{Recommended Citation}

$\mathrm{Xu}$, Xueyan, "Prediction of life -threatening events in infants using heart rate variability measurements" (2002). Graduate Theses, Dissertations, and Problem Reports. 2432.

https://researchrepository.wvu.edu/etd/2432

This Dissertation is protected by copyright and/or related rights. It has been brought to you by the The Research Repository @ WVU with permission from the rights-holder(s). You are free to use this Dissertation in any way that is permitted by the copyright and related rights legislation that applies to your use. For other uses you must obtain permission from the rights-holder(s) directly, unless additional rights are indicated by a Creative Commons license in the record and/ or on the work itself. This Dissertation has been accepted for inclusion in WVU Graduate Theses, Dissertations, and Problem Reports collection by an authorized administrator of The Research Repository @ WVU.

For more information, please contact researchrepository@mail.wvu.edu. 


\title{
Prediction of Life-Threatening Events in Infants Using Heart Rate Variability Measurements
}

\author{
Xueyan Xu \\ Dissertation submitted to the \\ College of Engineering and Mineral Resources \\ at West Virginia University \\ in partial fulfillment of the requirements \\ for the degree of
}

Doctor of Philosophy

in

Electrical Engineering

Stephanie Caswell Schuckers, Ph.D., Chair

Eugene V. Cilento, Ph.D.

Wils L. Cooley, Ph.D.

Mark A. Jerabek, Ph.D.

Michael E. Schuckers, Ph.D.

Lane Department of Computer Science and Electrical Engineering

Morgantown, West Virginia

2002

Keywords: Heart Rate Variability, Apparent Life-Threatening Events, Sudden Infant Death Syndrome, RR interval, Artifact, and Classification.

Copyright 2002 Xueyan Xu 


\section{ABSTRACT \\ Prediction of Life-Threatening Events in Infants Using Heart Rate Variability Measurements}

\section{Xueyan Xu}

Sudden infant death syndrome (SIDS) is the sudden death of an infant under 1 year old without any apparent sign ahead of time. It is the leading cause of death of infants between the age of one month and one year in the developed countries. After thirty years substantial worldwide research, it is still impossible to predict or prevent SIDS. With the long-term goal to prevent SIDS, this study explores prediction of life-threatening events using heart rate variability (HRV). HRV is an indirect measure of the autonomic nervous system input to the cardiovascular system. The hypothesis is that infants who are at high risk for life-threatening events and SIDS have a lack of nervous system control. Although statistically significant differences in HRV have been found between infants at high risk for SIDS and controls in previous studies, few studies go one step further to classify the infant group or put effort toward prediction of future events for a specific infant.

We investigate whether HRV can differentiate infants at risk for future apparent life-threatening events (FALTE) using the dataset collected by the Collaborative Home Infant Monitoring Evaluation (CHIME) study group. To achieve this, we developed and validated an artifact rejection routine, used statistical methods to study differences between normal and FALTE infant groups, and explored the relationship between HRV parameters. Lastly, the major work of our study is to design a model to classify infants using HRV, including logistic regression, decision tree, and neural networks. Promising results (67\% sensitivity and $100 \%$ specificity) are achieved for the test dataset by varying the model parameters and the threshold. Up until now, there is little information available for determining the likelihood of future life-threatening events for a newborn. If prediction at this accuracy level can be achieved through the measurement of HRV, infants at risk for life-threatening events can be identified and assisted by offering corresponding therapy. 


\section{ACKNOWLEDGEMENTS}

I would like to express my deepest gratitude and appreciation to my advisor, Dr. Stephanie Schuckers, for her undying patience, confidence, encouragement, and valuable suggestions throughout this research. Thank you for giving tirelessly of your time and expertise, your smart ideas, and guiding hand in helping me achieve my goal. Also, thanks for leading me into this wonderful biomedical world, in which I would love to develop my future career. You are my advisor in every way.

I would like to thank my committee members: Dr. Eugene Cilento, Dr. Wils Cooley, Dr. Mark Jerabek, and Dr. Michael Schuckers, for the benefit of their collective wisdom, helpful comments, and generously sharing their time and talents to guide me through the doctoral program.

I would like to give thanks to my colleagues in Biomedical Signal Analysis Laboratory, Pisut Raphisak, Reza Derakhshani, Amit Diggikar, and Limin Wang, for their assistance, encouragement and tolerance.

Last, but not least, special thanks to my family. I would like to thank my husband and my son, for their love, support, and patience. Many thanks to my parents-in-law, for their personal sacrifices and assistance during the hardest time of my Ph.D. study. No words can express my thanks to my parents and brother, for their endless love and support. Special thanks to my father, who taught me to value education, always encourages me to pursue higher goals, and has been a constant source of encouragement. 


\section{TABLE OF CONTENTS}

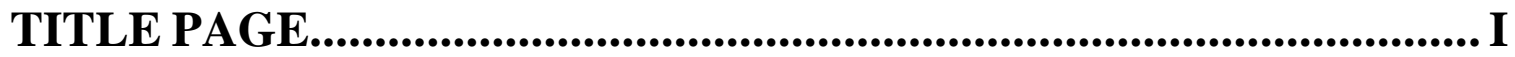

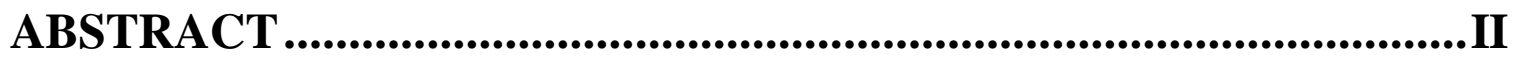

ACKNOWLEDGEMENTS................................................................... III

LIST OF FIGURES ............................................................ VIII

LIST OF TABLES .............................................................. VII

\section{CHAPTER}

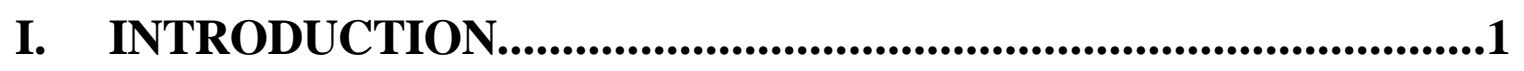

1.1 Sudden Infant Death Syndrome …………………................................... 2

1.2 Relationship between SIDS and Apnea/Bradycardia .......................................... 4

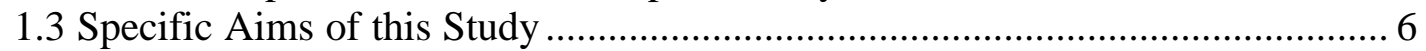

\section{HEART RATE VARIABILITY...........................................11}

2.1 The Heart and the Nervous System ............................................................... 12

2.2 Heart Rate and Heart Rate Variability

Noninvasive Methods of Measuring Autonomic Tone ...................................... 16

2.3 Heart Rate Variability as a Predictor of SIDS and Apnea/Bradycardia ............ 19

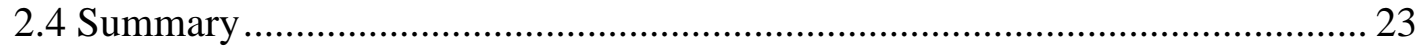

\section{MEASUREMENT OF HEART RATE VARIABILITY...............25}

3.1 Time - Domain (Descriptive Statistics) …………………………………….... 28

3.2 Frequency - Domain........................................................................................ 31

3.3 Time - Frequency - Domain .............................................................................. 34

3.3.1 Concept of wavelet transform ................................................................. 35

3.3.2 Time-frequency measurements of heart rate variability ............................ 37

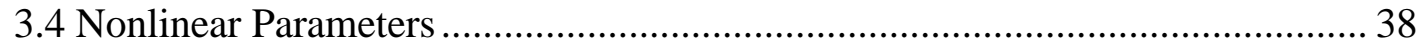

3.4.1 Approximate Entropy (ApEn) …………………………………........ 39

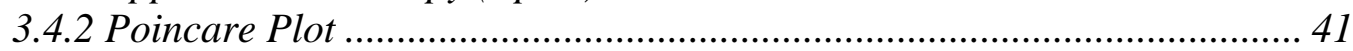

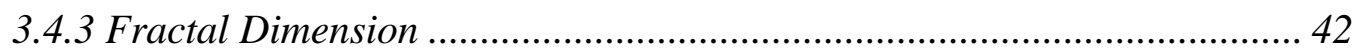

3.5 Considerations in HRV Measurements.............................................................. 43

3.5.1 Selection of appropriate analytical epochs............................................. 43

3.5.2 Overlapped or non-overlapped segments................................................ 45 
3.5.3 Effect of sleep state on heart rate variability.......................................... 46

3.6 Results of HRV Calculation for the Infant Dataset ....................................... 47

3.6.1 Statistical summary of infant HRV ................................................... 49

3.6.2 Differences in HRV across sleep states ................................................. 52

3.6.3 Differences in HRV between two infant groups...................................... 53

3.6.4 Changes of HRV with post conceptional age in infants........................... 55

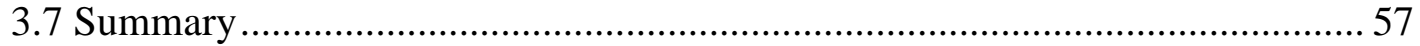

\section{ARTIFACTS IN THE HEART PERIOD DATA........................59}

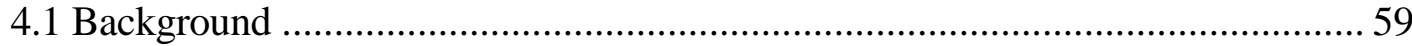

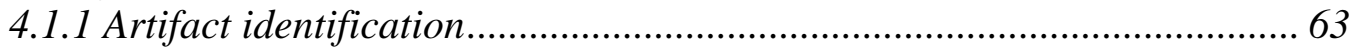

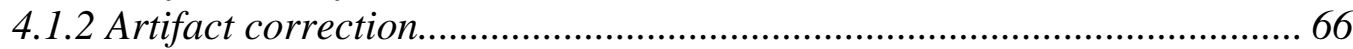

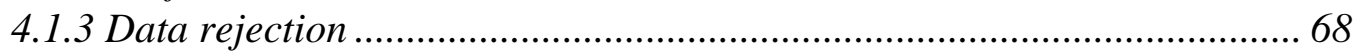

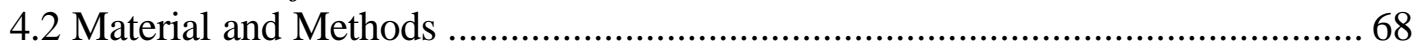

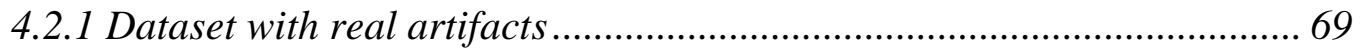

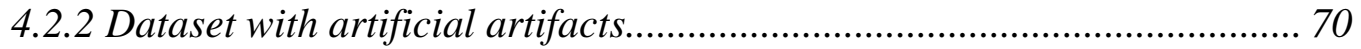

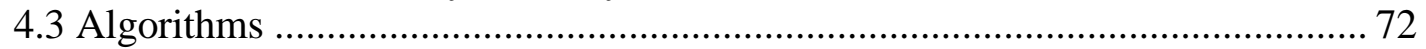

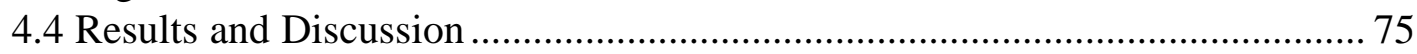

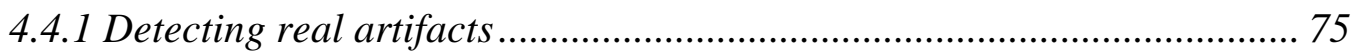

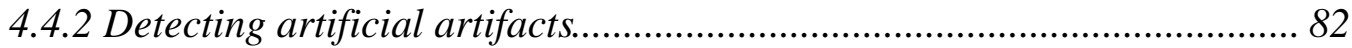

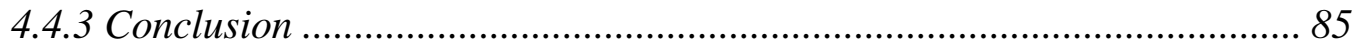

4.5 Evaluating HRV Parameters in the Presence of Noise ...................................... 86

4.5.1 Effect of artifacts on different HRV parameters ..................................... 87

4.5.2 Effect of specificity on HRV changes ..................................................... 88

4.5.3 Effect of type 4 \& 5 real artifacts on HRV changes............................... 89

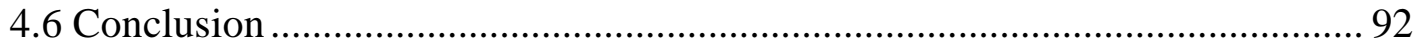

\section{RELATIONSHIP OF HEART RATE VARIABILITY}

MEASURES..................................................................94

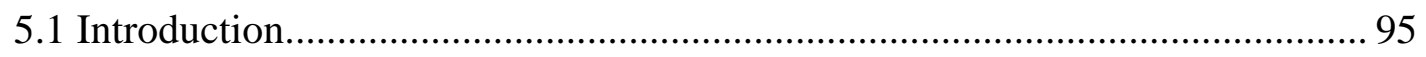

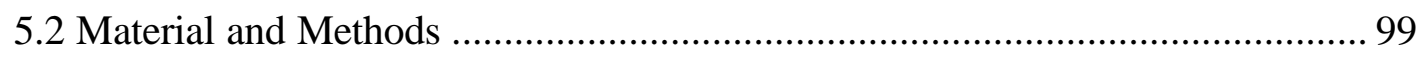

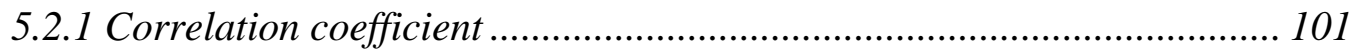

5.2.2 Correlation from individuals ............................................................ 102

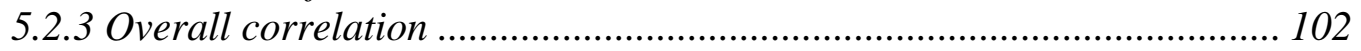

5.2.4 Overall correlation with infant group taken into consideration............. 103

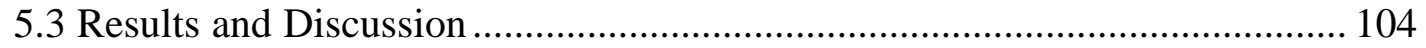

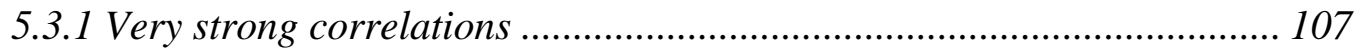

5.3.2 A highly correlated group ................................................................. 108

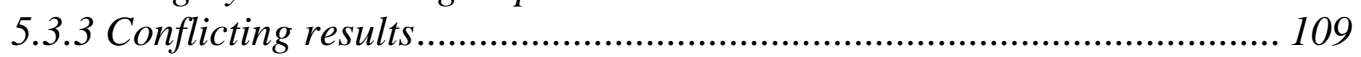

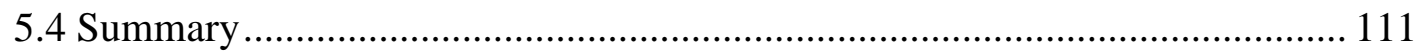




\section{PREDICTION OF SUBSEQUENT LIFE-THREATENING EVENTS IN INFANTS USING HEART RATE VARIABILITY113}

6.1 Basic Concept of Classification Models …………………………………....... 114

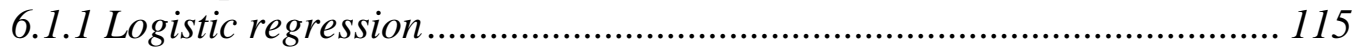

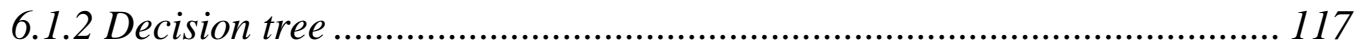

6.1.3 Artificial neural network.................................................................... 120

6.1.4 Comparison of the three models ........................................................ 127

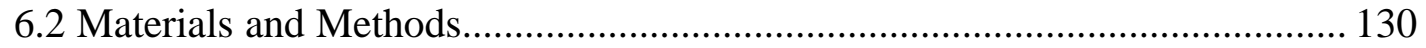

6.2.1 Event classification ……………….................................................. 130

6.2.2 Training and testing datasets.......................................................... 132

6.3 Classification of Infants using SAS Enterprise Miner..................................... 134

6.3.1 Insight into the input data.................................................................. 135

6.3.2 Logistic regression ............................................................................ 137

6.3.3 Decision tree ……………………...................................................... 139

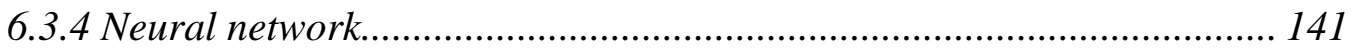

6.3.5 Comparison of three models ................................................................. 142

6.3.6 Classification of infants ..................................................................... 144

6.4 Classification of Infant Group Using a Neural Network Toolbox in Matlab .. 146

6.4.1 Materials and methods................................................................... 147

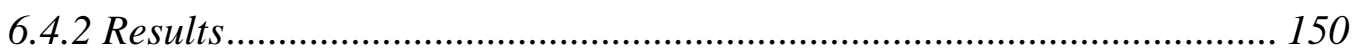

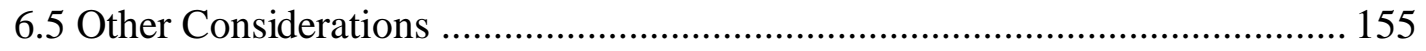

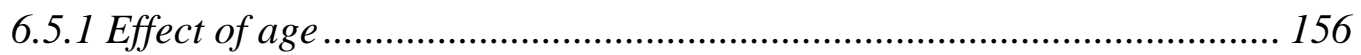

6.5.2 Utilization of age distribution information ............................................. 159

6.5.3 Age matching of the infants in event and control groups ........................ 160

6.5.4 Re-randomize the training and testing datasets..................................... 164

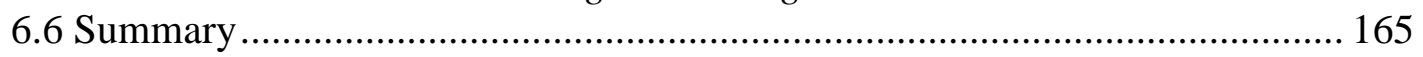

\section{CONCLUSION AND FUTURE WORK..................................167}

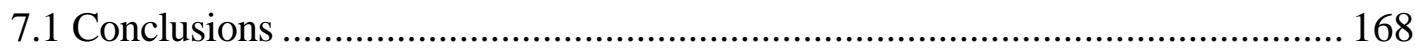

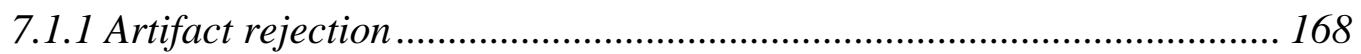

7.1.2 Correlation of HRV measurements...................................................... 169

7.1.3 Prediction of life-threatening events................................................. 170

7.2 Limitations of the Study and Future Work ...................................................... 171

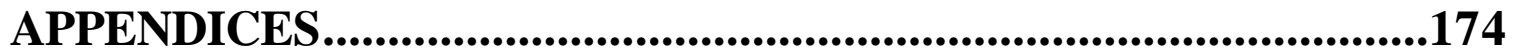




\section{LIST OF FIGURES}

Figure 2.1 Structure of the heart …………………….............................................. 12

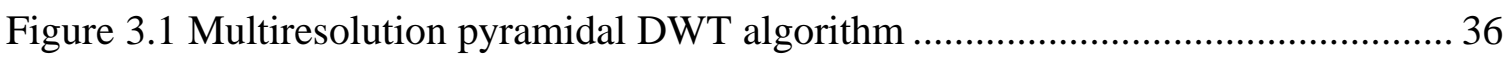

Figure 3.2 Poincare plot of 5-min RR intervals of infant 30166 .................................... 42

Figure 3.3 RR intervals in three sleep states for one infant ......................................... 47

Figure 3.4 Example of four HRV parameters ............................................................. 48

Figure 3.5 Sleep state differenced in HRV of the 122 infants ........................................ 50

Figure 3.6 Changes of HRV with post conceptional age ……….................................... 55

Figure 3.7 Distribution of PCA for event and control infants ......................................... 57

Figure 4.1 Effect of one missing beat on the power spectral density ...............................62 62

Figure 4.2 Six types of artifact in our data................................................................. 70

Figure 4.3 Sensitiveness of the algorithm to its own failure.......................................... 73

Figure 4.4 Sensitivity, specificity and accuracy of the six algorithms ............................ 80

Figure 4.5 ROC curve which compares various algorithms ............................................ 81

Figure 4.6 Changes of sensitivity \& specificity of various algorithms............................ 84

Figure 4.7 Effect of two scattered missing beats on HRV parameters ............................ 88

Figure 4.8 Effect of false positives of the artifact detection algorithm on HRV .............. 89

Figure 4.9 Effect of type $4 \& 5$ artifacts and algorithm $\mathrm{f}$ on HRV ................................... 91

Figure 5.1 Median of correlation coefficients for QS ............................................... 106

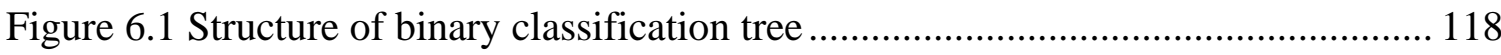

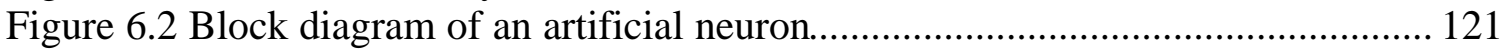

Figure 6.3 Multilayer feedforward neural network...................................................... 122

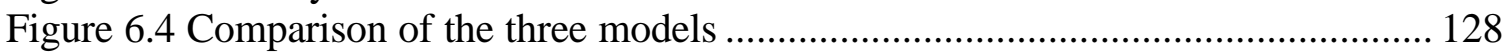

Figure 6.5 Distributions of Median, SDNN, and ApEn............................................... 136

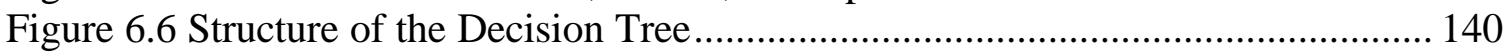

Figure 6.7 Diagram for the comparison of three classification models .......................... 142

Figure 6.8 ROC curve of the models in classifying all 5-min segments ........................ 143

Figure 6.9 ROC curve of three classification models for infant classification............... 145

Figure 6.10 ROC curve of neural network model for reduced input set......................... 154

Figure 6.11 ROC curve of neural network model with age as an input .......................... 157

Figure 6.12 ROC curve of the models in classifying all 5-min segments ...................... 158

Figure 6.13 ROC curve of the models in classifying all infants ................................... 158

Figure 6.14 ROC curve of neural network model for infants less than 45.6 weeks ....... 160

Figure 6.15 ROC curve of neural network model for age matched control and event

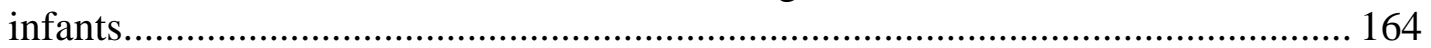

Figure 6.16 ROC curve of neural network model for re-randomized training and testing datasets 


\section{LIST OF TABLES}

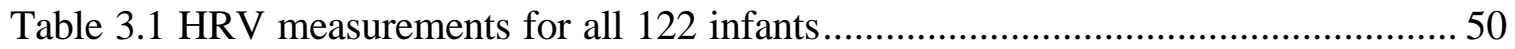

Table 3.2 HRV measurements for the two groups of infant ...................................... 51

Table 3.3 Correlation coefficients between PCA and HRV parameters.......................... 56

Table 4.1 Truth table for the definition of sensitivity, specificity, and accuracy ............ 75

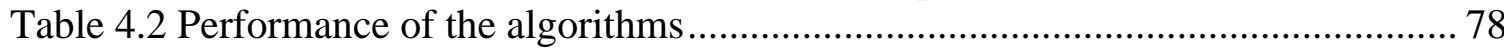

Table 4.3 Sensitivity, specificity and accuracy of the six algorithms ............................ 79

Table 5.1 Median of the correlation coefficients of the all infant in QS ...................... 105

Table 5.2 Median of the correlation coefficients of the all infants in AWK ................. 105

Table 6.1 Statistical summaries of the input interval variables ................................... 135

Table 6.2 Estimates of the Logistic Regression model............................................. 138

Table 6.3 Results of the regression model in classifying 5-min segments .................... 138

Table 6.4 Results of the tree model in classifying 5-min segments.............................. 139

Table 6.5 Results of the neural network model in classifying 5-min segments ............. 142

Table 6.6 Misclassification rate in detecting 5-min segments ..................................... 143

Table 6.7 Results for classifying all 5-min segments of the infants ............................. 151

Table 6.8 Results for classifying all of the infants............................................... 152

Table 6.9 Age and group information of event infants with matched controls .............. 162

Table 6.10 HRV measurement for the 36 age matched event and control infants ......... 163 


\section{CHAPTER I}

\section{INTRODUCTION}

The motivation for this dissertation is a disease called sudden infant death syndrome (SIDS), which is the sudden death of an infant under one year old without any apparent warning signs. It is the leading cause of death of infants between the age of one month and one year in developed countries. Although there is substantial worldwide research done in this area, the mechanism of SIDS remains unknown. There are still no proven risk factors to identify or predict the infant vulnerable to SIDS. Furthermore, the relationship between apparent life-threatening events (ALTE) and SIDS requires further study [WIL89, VER93]. Previous and future efforts will continue to be put into this area to stop such tragedies. Research is still focused on investigating the mechanism of the disease, examining the differences in physiological signals between healthy and SIDS infants, and finding the relationship between SIDS and apparent life-threatening events.

In this study, the differences in heart rate variability (HRV) between healthy infants and infants with future apparent life-threatening events (FALTE) are examined. Lower heart rate variability is associated with less control of the autonomic nervous system on heart and has been observed in SIDS and ALTE infants. Classification methods are investigated for their ability to predict future life-threatening events using heart rate variability. 


\subsection{Sudden Infant Death Syndrome}

Sudden Infant Death Syndrome (SIDS) is defined as the unexpected sudden death of an infant that cannot be explained by prior medical history or by postmortem examination [BER70, WIL89]. It was introduced as a specific disease entity into the International Classification of Diseases in 1972 [POE94]. Despite more than 20 years of extensive worldwide research, SIDS is still the leading cause of death of infants between the age of one month and one year in the developed countries. Benefit from the Back to Sleep Campaign started in 1992, where the SIDS death rate decreased from 1.2 to 0.8 per 1000 births from 1991 to 1995 . However, there are still about 3000 infants who die of SIDS every year in the United States [JOB01].

Results of previous studies indicate some characteristics and risk factors of SIDS. For instance, the peak incidence for SIDS is 24 months of age, siblings of SIDS are at greater risk of SIDS than nonsiblings, virtually all SIDS deaths occur during sleep, and it is highly recommended to avoid the prone sleeping position [HOF88, WIL89, PET80, PET86]. Potential risk factors for SIDS commonly include low birth weight, intrauterine growth retardation, premature birth, parental smoking, over-heating, not breastfeeding, young maternal age, and birth order - a greater risk for infant born third or higher in the birth order [BER72, HOF88, KRA89, NAE76]. In addition, although it is widely accepted that the ultimate cause of death in SIDS is attributed to failure in autonomic cardiorespiratory control, the specific circumstances leading to this fatal cardiorespiratory event remains undetermined. In other words, despite hundreds of studies on SIDS in recent years, no clear risk factor yet exists for identification of vulnerable infants. 
Clinicians and researchers have put much effort into finding the mechanism failure of SIDS. Vertes and Perry indicated that abnormally elevated fetal hemoglobin level was found in SIDS infants. They explained in their theory that high hemoglobin level causes chronic hypoxia, which will depress respiration, especially during quiet sleep. This depressed respiration will produce more extreme hypoxia, resulting in further respiratory depression, and ultimately causing the infant's death due to the cessation of respiration [VER93]. Mrowka et al. suggested that infants suffering from bronchopulmonary dysplasia (BPD) are more at risk of dying from SIDS [MRO96]. They examine whether and how newborns suffering from BPD differ in heart rate control from normal infants. While, Baker and McGinty studied the death of hypoxic kittens. They experimentally induced hypoxia in kittens and found that hypoxia reduced REM sleep, and increased the risk of cardiorespiratory failure and death. From their study, they suggested that REM sleep might protect against cardiorespiratory abnormalities in infants, and reduced REM sleep might associated with high risk of sudden infant death [BAK77]. However, Cornwell et al. found an abrupt increase in REM (rapid eye movement) sleep in the high risk SIDS group, when they studied the difference of REM and QS (quiet sleep) durations in high risk SIDS and control infants [COR96].

Several important advances in SIDS research have been made in the past few years. It is well known that prone sleeping position substantially increases the risk for SIDS, as well as exposure to smoking. Among these findings, the most theoretically important finding is that there are a variety of cardiac, breathing and sleep-state differences between SIDS victims and healthy infants as early as the first week of life [HAR98]. With the help of improved physiological signal monitoring and recording 
systems, it is feasible to obtain physiological signals during death or near-death events of the infants. Kelly et al. reported that they recorded signals of heart rate and breathing immediately before and during death in three infants, when they observed a decrease in heart rate before the cessations of breathing in all cases [KEL91]. Therefore, a common focus for research into the mechanism of SIDS is to study the difference in cardiorespiratory signals between normal infants and aborted-SIDS infants.

Researchers and clinicians are increasingly involved in the diagnostic evaluation of the risk of SIDS for healthy newborn, preterm infants or infants having exhibited apparent life-threatening events (ALTE), and infants of drug-addicted mothers. They found no evidence that some specific infant groups are at higher risk of sudden death, even though ALTE, preterm, and SIDS siblings are currently defined as risk groups. Presently, computer based polysomnographic recordings are used to assess the risk of SIDS [BAR94]. More research in this and other types of diagnostic or preventative monitoring are needed to its potential benefits.

\subsection{Relationship between SIDS and Apnea/Bradycardia}

The relationship between sudden infant death syndrome (SIDS) and apparent lifethreatening events (ALTE), including apnea and bradycardia, is still not clear. Bradycardia is a significant slowing of the heart rate and apnea is a cessation of breathing for an extended period. Because our study focuses on ALTE, this is an important consideration. Several studies have looked at this relationship. There is a suggestion that prolonged apnea and bradycardia are markers for the vulnerable infant to SIDS [KEL79,

KEL80, KEL82, KEL91, MEN94], while other studies are unclear [RAM01, JOB01]. 
Since access to data from infants who have died of SIDS is limited, many researchers choose to compare the differences in heart rate variability between normal infants and infants with life-threatening events. Since many previous studies link ALTE with changes in $\mathrm{HRV}$, it provides the basis for the hypothesis that it is possible to predict which infants will go on to have apparent life-threatening events. Analyzing the physiological signals of ALTE infant could be an effective method for SIDS study.

In the early 1970s, Steinschneider reported that among five infants who died from SIDS, two of them had prolonged sleep apnea [STE72]. Since then, there is a tendency to believe that there is a close relationship between apnea and SIDS. However, the results of apnea studies in infants at risk of SIDS are not consistent and even conflict. Kelly et al observed both prolonged sleep apnea and excessive amounts of periodic breathing in some of the 'near-miss' SIDS infants [KEL79], and also increased number of brief apneic pauses and more periodic breathing during QS in SIDS siblings [KEL80, KEL82]. Kejariwal et al. also found that prolonged apneas and changes in heart rate are associated with SIDS [KEL86]. But, results from the studies done by Schechtman, and Hoppenbrouwers et al showed significantly fewer apneic pauses in both QS and REM for SIDS victims [SCH91, HOP80]. Southall concluded from their results that there is no difference in the number of apneic pauses or periodic breathing between SIDS siblings and normal infants [SOU87].

In two SIDS studies [KEL91, MEN94], which reported a total of nine deaths, the researchers were able to obtain a record of the physiological signals during death or neardeath events. They all found decreased heart rate before the cessation of respiration. From their findings in these physiological data, the authors concluded that bradycardia in 
these infants seemed to be the result of prolonged severe hypoxemia, or obstructive apnea, or both. They believed that it is important to detect bradycardia in order to prevent sudden death in infants. Kelly et al. also found SIDS victims had more frequent episodes of bradycardia than normal infants [KEL86].

There is another critical problem that affects SIDS study. Several SIDS cases in previous studies were not found to be SIDS, but the result of infants murdered by their parents [FIR97]. The physiological data from those infants should not be expected show any characteristics of SIDS.

Despite the disagreement in how apnea or bradycardia are correlated with SIDS, most researchers would agree that infants who suffer from apparent life-threatening events, including apnea and bradycardia, are an important group to study. Finding the differences in the cardiorespiratory signals between normal infant and infants at risk for apparent life-threatening events might be helpful in the prevention of SIDS. Heart rate and heart rate variability are two of the commonly used cardiorespiratory signals for studies in this area.

\subsection{Specific Aims of this Study}

Previous studies have indicated that most SIDS victims suffer from cardiorespiratory failure, which reflects immaturity of autonomic nervous system (ANS) control. Studying physiological signals of infants could provide insight into detecting who is at risk for SIDS. Respiratory behavior, duration of rapid eye movement (REM) / quiet (QS) sleep, QT interval, and heart rate/heart rate variability have all been used to study the difference between potential SIDS victims and control infants. Among these 
cardiorespiratory signals, heart rate and heart rate variability (HRV) are the most commonly used variables. HRV has been shown to be a noninvasive and inexpensive way to assess the autonomic nervous system. Increased heart rate and decreased heart rate variability have been observed in SIDS victims [MAT92, SCH89, LEI80].

While it is unclear whether there is a relationship between SIDS and infants who have life-threatening events [RAM01, JOB01], there is a desire to gain a better understanding of the nature of ALTE, their frequency and severity, and the ability of home monitors to detect events and provide an alarm. The Collaborative Home Infant Monitoring Evaluation (CHIME) study group sought to study these issues through the collection of a massive clinical database [HOP96, NEU01, RAM01]. The database included physiological signals recorded in polysomnographic (PSG) studies and from a specially designed home monitor. Infants were classified into four different groups upon entrance into the study, healthy, preterm, SIDS siblings, and ALTE (infants with apparent life-threatening events). These infants were then studied for several months using a home monitor which recorded life-threatening events including bradycardia and apnea. Therefore, a classification can be generated regarding which infants had apparent lifethreatening events [RAM01].

Given the wealth of information linking SIDS/ALTE and heart rate variability (HRV), we desire to investigate whether heart rate variability is an effective predictor for future life-threatening events. Many methods have been used previously to study the difference in HRV between SIDS/aborted SIDS/ALTE infants and the control. Most of the studies focused on finding whether there is significant difference between those two kinds of infants in HRV. Statistically significant differences were found in most of these 
studies. However, there are few studies which attempt to classify or predict which infants are normal or at risk. In the study for this dissertation, heart rate variability is the physiological signal that we have focused on. Besides statistical analysis of HRV for healthy and ALTE infants, we go one step further to determine whether it is possible to classify infants with regard to apparent life-threatening events (ALTE) using HRV parameters. The following gives the specific aims and hypotheses of our research.

Specific Aim 1: Development and Comparison of Automatic Artifact Detection Algorithms, and Investigation of the Effect of Artifacts on HRV Measurements

- The differences between normal $R R$ and artifactual $R R$ will allow us to detect artifacts in RR time series automatically to an accepted accuracy level. The effect of artifacts on HRV measurements varies. Some noise sensitive HRV parameters should be avoided under noisy condition.

\section{Specific Aim 2: Determination of the Correlation between HRV Measurements}

- Many of the numerous HRV parameters which have been developed are correlated and this information can assist in infant classification.

Specific Aim 3: Prediction of Subsequent Life -Threatening Events in Infants Using Classification Trees, Neural Networks, and Logistic Regression with HRV as Inputs

- Heart rate variability in normal healthy infants is higher than for those infants who will go on to have serious life threatening events, such as apnea or bradycardia, and these differences will allow us to classify infants through sophisticated pattern recognition techniques.

We investigate whether $\mathrm{HRV}$ can differentiate infants at risk for future apparent life-threatening events (FALTE) using the CHIME dataset. To achieve this, we first 
developed and validated an artifact rejection routine. Second, statistical methods are used to determine if there are significant differences in HRV between normal and FALTE infant groups. We next studied the relationship between HRV parameters and all the affected factors, which might provide a clearer understanding of the parameters, and help determine how to use HRV as a classifier in the infant group prediction problem. Lastly, the major work of our study is to design a model to classify infants using HRV. Although statistically significant differences in HRV have been found between infants at high risk for SIDS and controls, few studies go one step further to classify the infant group or put effort toward prediction of future events for a specific infant. We work toward this direction using various prediction models including logistic regression, decision tree, and neural networks.

Chapter 2 of this dissertation describes the concept of heart rate variability, and previous work including differentiation of SIDS/ALTE infants from the control. Chapter 3 is an overview of the common HRV measurements, including time domain, frequency domain, time-frequency domain, and nonlinear methods, as well as considerations in HRV calculation. In addition, the CHIME dataset, on which this study is based, and the statistical analysis of the resultant HRV calculated for the CHIME infants are summarized in Chapter 3. Based on the above three specific aims, three different studies are performed. Chapter 4 is the artifact study, which provides an automatic algorithm to clean the RR interval data for further analysis. Chapter 5 evaluates the relationship between HRV parameters, which facilitates in the understanding of $\mathrm{HRV}$ and the selection of the most appropriate parameter for a specific application. Next, the possibility of using HRV to classify event versus control infants is investigated in 
Chapter 6 . These three chapters describe three independent studies, each having their own background introduction, methods, results and conclusion. However, these studies are also tightly linked toward the final goal for this dissertation, prediction of life-threatening event in infants using HRV. Artifact removal is necessary to make the raw RR interval data and derived HRV measurements more reliable. The correlation study assists in obtaining insight into the HRV parameters, and could be used to refine the classification model. Lastly, Chapter 7 summarizes the results, addresses the limitations of the infant classification study, and describes the future work.

In addition, some detailed results are shown in appendix. Appendix A is the users' guide for the integrated heart rate variability analysis tools, which were developed in MATLAB. Appendix B and C are results for the artifact study, and appendix D and E includes results from the correlation and classification studies. 


\section{CHAPTER II}

\section{HEART RATE VARIABILITY}

Heart rate and heart rate variability is one of the commonly used cardiorespiratory signals in studies of infants. The cyclical changes in sinus rate over time are termed Heart Rate Variability (HRV) [ORI92]. It is widely accepted hat cyclical variations of heart rate, arterial blood pressure and peripheral vascular resistance are under the control of the autonomic nervous system (ANS). Heart rate variability carries much information about the status of ANS, about the general health of the heart, and susceptibility to various cardiac diseases, including sudden cardiac death (SCD) and sudden infant death syndrome (SIDS). It can be considered a noninvasive and inexpensive way to analyze the autonomic nervous system.

Heart rate varies due to physical or mental stress, exercise, respiration, metabolic changes, and hormonal influences. Normal aging and some heart diseases also result in a decreased heart rate variability, which reflects the reduction of autonomic control of the heart. Heart rate and heart rate variability are determined by a complex interaction of the sympathetic and parasympathetic divisions of the autonomic nervous system. In an intact, unblocked individual, the heart rate at any time represents the net effect of the parasympathetic (vagal) nerves which slow it and the sympathetic nerves which accelerate it [MAL95]. Therefore, a brief introduction of the structure of heart, its electrical activity, and the nervous system is necessary. 


\subsection{The Heart and the Nervous System}

The heart is a muscular organ of the human body which transports oxygen and nutrient supply to the tissues and removes byproducts of metabolism. There are four pumping chambers in the heart (Figure 2.1). The left atrium pumps newly oxygenated blood which is returned from the lungs to the left ventricle. The left ventricle then pumps the oxygenated blood through the aortic valve to the body to deliver oxygen and nutrients. The right atrium receives de-oxygenated blood from the body and pumps it into the right ventricle. The right ventricle fulfills the function of pumping blood through the pulmonary valve into the lungs for oxygenation [LEV90].

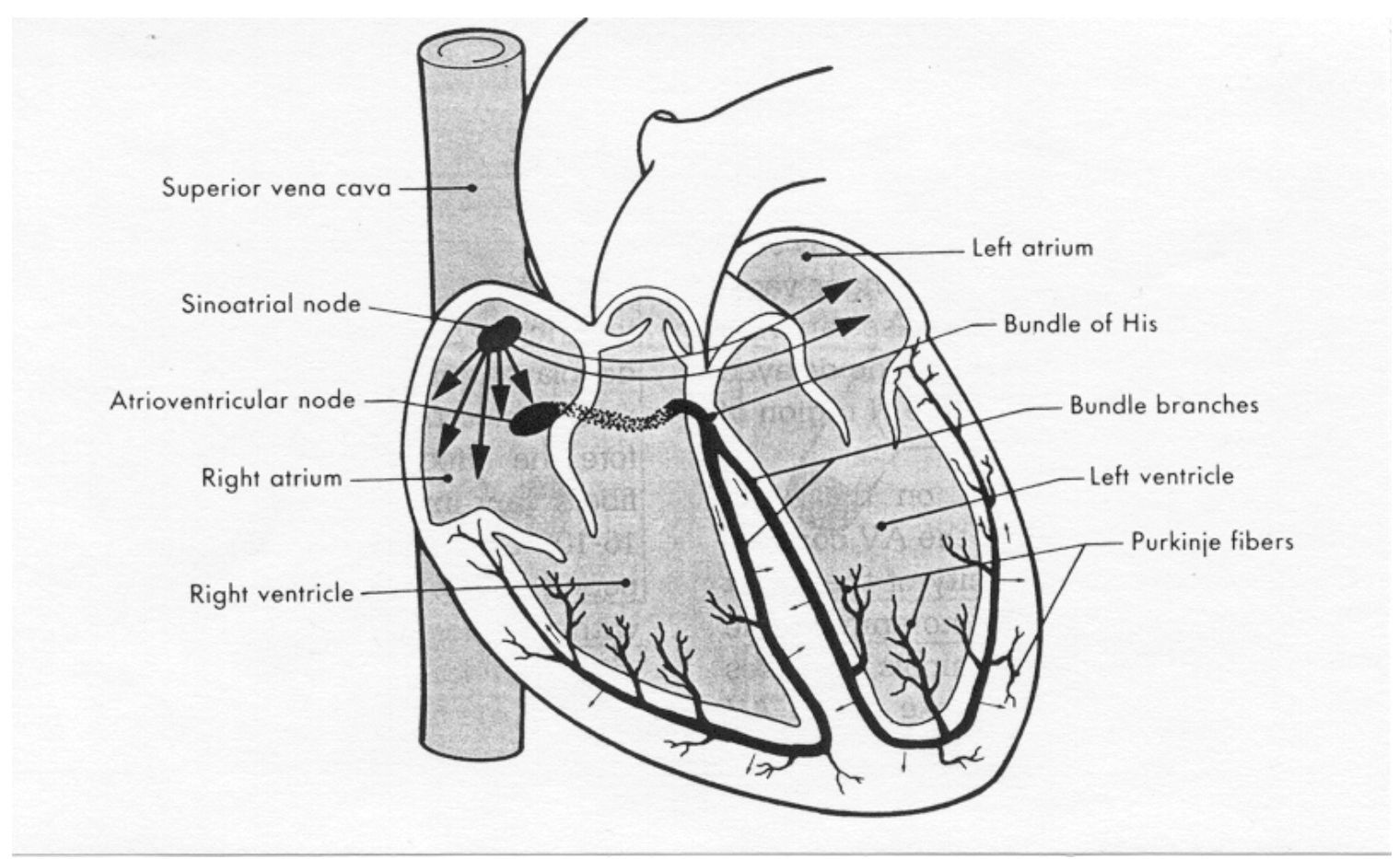

Figure 2.1 Structure of the heart

The heart achieves its pumping action through cardiac muscle contractions, which is driven by the electrical stimulation in the heart. The sinoatrial (SA) node, located in the 
right atrial wall near the opening of the superior vena cava, is the intrinsic pacemaker of the heart. It automatically generates an electrical depolarization, which is conducted throughout the atria and causes a contraction of the atrial muscle. The atrioventricular $(\mathrm{AV})$ node, located at the base of the right atrium near the septum, above the junction of the atria and ventricles, is the only electrical transmission link between atria and ventricles. It provides a delay of the depolarization from the atria, which allows the atria contract completely, and pump blood to the ventricle. Then, the depolarization is spread quickly through the His-Purkinje fibers in the ventricle, ensuring that the ventricle depolarizes and contracts consistently and pumps blood to the body or lung.

Heart rate is regulated by the autonomic control of the nervous system on the heart. The nervous system is organized into the central nervous system (CNS), consisting of the brain and spinal cord, and the peripheral nervous system (PNS), consisting of nerve fibers that carry information between the CNS and other parts of the body or periphery [SHE94]. The PNS is further subdivided into afferent and efferent divisions. The afferent division carries information to the CNS, informing it of the external environment and providing status reports on internal activities being regulated by the nervous system. Instructions about the desired effect are ransmitted from CNS via the efferent division to the organs. The efferent nervous system is divided into the somatic nervous system and autonomic nervous system. Somatic nervous system consists of fibers of motor neurons that supply the skeletal muscles. Autonomic nervous system innervates smooth muscle, cardiac muscle and glands, and it is further subdivided into the sympathetic nervous system and the parasympathetic nervous system, both of which innervate most of the organs handled by the autonomic system. 
Heart rate is determined primarily by autonomic influences on the sinoatrial (SA) node, which is normally the pacemaker of the heart because it has the fastest spontaneous rate of depolarization to an action potential threshold. Action potentials are the method by which the electrical stimulation of each muscle cell is spread through the heart. When the SA node reaches threshold, an action potential is initiated that spreads throughout the heart, inducing the heart to contract. The heart is innervated by both sympathetic and parasympathetic divisions of the autonomic nervous system, which modify the rate of contraction, even though nervous stimulation is not required to initiate contraction. The parasympathetic nerve to the heart, the vagal nerve, primarily supplies the atrium, especially the SA and AV nodes. There are no significant parasympathetic innervations of the ventricles. The cardiac sympathetic nerves also supply the atria, including the SA and AV nodes, and richly innervate the ventricles as well. Parasympathetic and sympathetic stimulation have the different effects on the heart.

The parasympathetic nervous system's influence on the SA node is to decrease the heart rate. Because parasympathetic stimulation decreases the rate of depolarization to threshold, the SA node reaches threshold and fires less frequently. The effect of parasympathetic stimulation of the AV node is to decrease the node's excitability, prolonging transmission of impulses to the ventricles even longer than the usual AV nodal delay. The effect on atrial contractile cells is to shorten the action potential. As a result, atrial contraction is weakened. These actions are appropriate considering that the parasympathetic system controls heart action in quiet, relaxed situations, when the body is not demanding an enhanced cardiac output. 
In contrast, when there is a need for greater blood flow, the sympathetic nervous system, which controls heart action in emergency or exercise situations, speeds up the heart rate through its effect on the pacemaker tissue. The main effect on sympathetic stimulation of the SA node is to increase its rate of depolarization so that threshold is reached more rapidly. Sympathetic stimulation of the AV node reduces the AV nodal delay by increasing conduction velocity. It similarly speeds up the spread of the action potential throughout the specialized conduction pathway. In the atrial and ventricular contractile cells, both of which have an abundance of sympathetic nerve endings, sympathetic stimulation increases contractile strength so that the heart beats more forcefully and squeezes out more blood. The overall effect of sympathetic stimulation on the heart, therefore, is to improve its effectiveness as a pump by increasing the heart rate, decreasing the delay between atrial and ventricular contraction, decreasing conduction time throughout the heart, and increasing the force of contraction.

Parasympathetic and sympathetic effects on heart rate are antagonistic. At any moment, the heart rate will be determined largely by the existing balance between the inhibitory effects of the parasympathetic nerve and the stimulatory effects of the cardiac sympathetic nerves. Under resting conditions, parasympathetic discharge is dominant. Heart rate is increased by simultaneously increasing sympathetic and decreasing parasympathetic activity. The relative level of activity in these two autonomic branches to the heart, in turn, is primarily coordinated by the cardiovascular control center located in the brain stem, in the medulla [SHE94]. 


\subsection{Heart Rate and Heart Rate Variability}

\section{--- Noninvasive Methods of Measuring Autonomic Tone}

As described above, heart rate and heart rate variability are the result of a complex interaction of the sympathetic and parasympathetic divisions of the autonomic nervous system. They have been used as indices of autonomic integrity in the fetus, infants, and adults in many studies. The first documented observation of HRV is often credited to Hales (1733), who observed a respiratory pattern in the blood pressure and pulse of a horse [BER97, ORI92, SPI93, PIC99]. Most of the early studies focused on respiratory sinus arrhythmia (RSA). Afterwards, research on HRV moved in two directions. One of them is toward understanding the physiological mechanisms controlling heart rate rhythms. The other is to investigate specific relationships between HRV and clinical status [BER97]. The clinical importance of HRV was acknowledged in the late 1980s, when many studies confirmed that HRV was a strong and independent predictor of mortality after an acute myocardial infarction [TASK96]. However, researchers have different understandings of how heart rate (HR) or heart rate variability (HRV) reflects the activity of the sympathetic and parasympathetic nervous system.

To test the hypothesis that HRV is highly correlated with mortality in patients after acute myocardial infarction, Kleiger et al. analyzed heart rate data of 808 patients who survived after an acute myocardial infarction. They defined HRV as the standard deviation of all normal RR intervals in a 24-hour ECG recording made $11 \pm 3$ days after an acute myocardial infarction. They found the relative risk of mortality in the group with less than 50ms HRV was 5.3 times higher than the group with more than $100 \mathrm{~ms}$ HRV. 
They explained that decreased HRV is correlated with increased sympathetic or decreased vagal tone and may cause ventricular fibrillation [KLE87].

It is also hypothesized that there is a relationship between autonomic activity and vulnerability of the heart to ventricular arrhythmias. HRV, which was used to evaluate the influence of the autonomic nervous system, also has been proposed as a tool for identifying persons at high risk of sudden cardiac death (SCD). In one study, Myers et al. performed frequency analysis of HRV and found that two frequency components (C: 0.15-0.35 Hz, D: $0.35-0.50 \mathrm{~Hz}$ ) are able to separate the SCD group and non-SCD group [MYE86].

HRV is also used in the clinical study of chronic congestive heart (CHF) disease. In [SAU88], power spectral analysis of HRV was used to evaluate autonomic control in CHF patients and differentiated CHF patients from control subjects. They found HRV spectral power distinctly decreased at all examined frequencies $(0.01 \mathrm{~Hz}$ to $1.0 \mathrm{~Hz})$ in $\mathrm{CHF}$ patients. Their finding suggested that there is diminished vagal, but relatively preserved sympathetic influence on HR in CHF patients.

Landes et al. examined the maturation of the central nervous system of infants by studying the changes in HR and HRV across conceptional ages and sleep-wake states. Traditional statistical analysis (mean, variance, and range) and two/three dimension plot (histogram and Poincare plot) were used in the analysis. They define a method to compute the amount of scatter or dispersion of the 2-D Poincare plot, in order to do the comparison quantitatively. The results showed that mean HR was very dependent on sleep state, and HRV increased with age (the maturation of the central nervous system) for all sleep-wake states. They also showed that HRV analysis methods are effective in 
characterizing the HRV features that reflect different sleep states and maturational levels in infants [LAN96].

One of the important studies in the current literature on HRV analysis is the sympathovagal balance. The sympathovagal balance can be defined from the classical Rosenblueth-Simeone model, which describes the effect of a combination of sympathetic and vagal influences on the sinus node [ROS34]. According to the Rosenblueth-Simeone model, the actual HR is proportional to the sympathovagal balance. For an individual person, $H R=m n \cdot H R_{0}$, where $H_{0}$ is the intrinsic heart rate, which is a person-dependent constant, $\mathrm{m}$ is the sympathetic factor and $\mathrm{n}$ is the vagal fictor. This multiplicative relation represents the antagonism of sympathovagal interaction adequately. The value of $\mathrm{m} \cdot \mathrm{n}$ may be defined as sympathovagal balance. $m \cdot n>1$ means sympathetic predominance, while $m \cdot n<1$ means vagal predominance. Since the person-dependent constant $\mathrm{HR}_{0}$ cannot be determined noninvasively, Bootsma et al., assumed that normalized low frequency power of $\mathrm{HR}$ can be used as a noninvasive alternative. They studied the individual LF - HR relation during incremental head-up tilt in health young male to demonstrate the above assumption. From the result showed a linear relation between LF and HR. This confirms that heart rate variability can be used as a noninvasive method to assess the sympathovagal balance [BOO94]. Pichot et al. applied both Fourier transform and wavelet transform to sequences of RR interval of six adults, who were injected with increased doses of atropine and propranolol, to study the sympathovagal balance. They found wavelet transform is a better way to quantify HRV, and then to assess the dynamics of the autonomous nervous system activity. It can detect the abrupt changes in autonomous tone precisely [PIC99]. 
Heart rate variability has been used as a powerful tool to evaluate the function of the autonomic nervous system, and to quantify sympathovagal balance. Furthermore, it can be used to predict sudden cardiac death, mortality after myocardial infarction, chronic congestive heart disease, and the maturation of the central nervous system of infants. It is also proposed as a potential predictor for SIDS and apnea/bradycardia.

\subsection{Heart Rate Variability as a Predictor of SIDS and Apnea/Bradycardia}

Infant heart rate and electrocardiogram (ECG) data have been used for many years in the study of Sudden Infant Death Syndrome (SIDS), based on the hypothesis that there is a primary failure in autonomic cardiorespiratory control for SIDS infants. Since the function of the autonomic nervous system is thought to be related to SIDS, finding a method to assess autonomic function in infants has become important. Many studies indicate that heart rate and heart rate variability are good indexes to assess the maturation and functionality of the autonomic nervous system in infants. These methods play an important role in the study of this area.

In order to determine whether there is any abnormality in the autonomic nervous system of infants, many researchers choose to compare the differences of heart rate and heart rate variability between normal infants and infants with life-threatening events or siblings of SIDS, which are considered to be the infants at risk for SIDS. Because SIDS presumably occurs during sleep and heart rate is influenced by the sleep state, studies of HR and HRV often distinguish sleep states first, and then measure and analyze the HR/HRV within different sleep states [LEI80, MRO96, MRO97, SCH89, SCH92, KLU88, PIN93, HAR78]. Harper et al. even indicate that during the first 6 months of life, 
sleep state related changes in HR/HRV exceed those due to the development of the autonomic nervous system [HAR78]. However, some papers do not consider sleep states [ANT90]. Usually, sleep-waking states are classified as quiet sleep (QS), rapid eye movement sleep (REM), awake (AWK), and indeterminate for each 30-sec or 1-min block.

In [LEI80], 5,000 to $10,000 \mathrm{RR}$ intervals recorded in REM and QS were used to study HRV in both aborted SIDS and normal infants. Median and interquartile range (IQR) were calculated using all of the artifact-free RR intervals. They found that at each age, and in both sleep states, Median(RR) and IQR(RR) are 5-10\% and 10-40\% smaller, respectively, for aborted SIDS infants than for normal infants. Their results showed that aborted SIDS infants have significantly shorter RR intervals and decreased overall and beat-to-beat variability in both REM and QS. Normalization did not change the significance of these differences. It is already known that heart rate and heart rate variability represent the net effect of sympathetic and parasympathetic activity. Faster heart rate in the aborted SIDS infants might be due to an increase in sympathetic activity, a decrease in parasympathetic activity, or a combination of both. They considered the result from previous research which found shorter QT intervals in aborted SIDS infants. Since parasympathetic activity has little effect on ventricular repolarization, which is indicated by the QT interval, they concluded that the faster heart rate in aborted SIDS infants may be the result of only an increase in sympathetic activity. Decreased overall HRV in the aborted SIDS infants maybe due to reduced fluctuations in sympathetic activity. On the contrary, Harper and coworkers attributed the increase in heart rate to a 
decreased vagal activity, since short-term variability is primarily under the control of vagal system [HAR78].

Mrowka et al. used low frequency (LF) power to high frequency (HF) power ratio (LF/HF) to characterize the sympathovagal balance, in a study of the HRV in normal infants and infants with broncho-pulmonary dysplasia (BPD). BPD infants were thought to be more at risk of SIDS. They defined 0.02 to $0.2 \mathrm{~Hz}$ as LF band, and 0.2 to $1.5 \mathrm{~Hz}$ as HF band. Their result showed significant difference between these two groups of infants for LF/HF ratio in both REM and QS [MRO96].

The 24-hour ECG was used to study the HRV difference between SIDS and normal infants in [SPI87]. They only used the RR intervals when breathing was relatively regular in amplitude and rate and not interrupted by sighs or apneic pauses. 120-sec duration episode was chosen for the frequency analysis on both $R R$ and $\Delta R R$. Through visual comparison of the spectrum, they did not find any clear difference between SIDS and normal infants. When they divided the spectrum into 25 frequency bands, significant difference was found in the frequency band at 6-8 beats per cycle.

Many papers indicate that aborted SIDS infants exhibit lower overall HRV during waking than normal infants. A study in [SCH89] attempts to determine which type or types of heart rate variation are reduced in SIDS infants by spectral analysis of HRV. The heart rate variation in three frequency bands was examined: respiratory sinus arrhythmia (RSA) (period $0.9 \sim 3 \mathrm{~s})$, mid-frequency $(4.0 \sim 7.5 \mathrm{~s})$, and low-frequency $(12 \sim 30 \mathrm{~s})$. They found that HRV in these three bands were all diminished in SIDS infants under one month of age during AWK and REM, but they did not find any heart rate-independent reduction in any type of $\mathrm{HRV}$ in QS. The hypothesis they made is that this sleep-state- 
dependence in HRV could indicate an abnormality of autonomic control mechanisms during AWK and REM in the SIDS infant.

Pincus et al. evaluated the heart rate dynamics of normal infants by approximate entropy (ApEn), and subsequently examined the capability of using ApEn to detect abnormal heart rate dynamics in aborted-SIDS infants. Their study is based on the hypothesis that the heart rate in aborted-SIDS infants is more regular (lower ApEn) but more unstable than normal infants. Since autonomic nervous system activity (which regulates heart rate dynamics) varies with sleep state, all their comparisons were performed during different sleep states. From the resultant ApEn values, they concluded that low ApEn values were associated with aborted-SIDS infants, because they found 5 of 14 aborted-SIDS infants had at least one ApEn value below the minimum ApEn value of 45 tested normal infant in QS. The results also showed fifty percent of aborted-SIDS infants had greater ApEn instability across quiet sleep, and REM is more regular than QS [PIN93].

In [ANT90], only RR intervals at regular breathing period were used in the analysis of HRV, and they did not take into consideration the sleep state. They found no significant differences between the SIDS infant and normal infants in terms of average heart rate, overall HRV and beat-to-beat HRV. Power spectral analysis of HRV also did not show any significant difference, although the HRV spectral densities of the SIDS group appeared lower than the normal infant for all frequencies up to $0.425 \mathrm{~Hz}$. Therefore, they felt that analysis of HRV did not differentiate SIDS infants from normal infants.

Many methods have been used to study the difference between SIDS/aborted SIDS/ALTE infants and the control. Most of the studies focused on finding whether there 
is statistical difference between those two kinds of infants in HRV. The methods used include the paired Wilcoxon test [MRO96], Welch test [LEI80], two-way analysis of variance [ANT90, KLU88], and one-way analysis of variance [SCH89]. Significant differences were found in almost all of the studies, even though conflicting results also exist [ANT90, VAL88].

Few studies have been done to classify or predict the infant group. The research in this dissertation attempts to classify infants based on heart rate variability and other parameters. One such study used artificial intelligence (AI) techniques in research of SIDS [VAL88], because AI is good for investigating complex medical problems with large sets of clinical and physiological data. Several HRV parameters, such as coefficient of variation, coefficient of variation of $\Delta \mathrm{RR}, \mathrm{RSA}$, respiratory rate and average values of HR were used as inputs to a rule-based expert system generator. The results showed that even though the trend of increased HR and decreased HRV in the SIDS infants was statistically non-significant, an expert system, which is based only on cardiorespiratory data, might be helpful in defining decision rules to identify SIDS cases.

\subsection{Summary}

Analyzing HRV is an effective approach to study the difference in cardiac signals between SIDS risk infants and control infants. It might be used as an independent predictor of SIDS. The difficulty with this research is that HRV is a complex and often ambiguous variable. It has numerous definitions as well as quantification methods. In most of the previous studies, researchers have chosen different HRV measures and used them on their own dataset. Different or even conflicting results of their studies might be 
because of using different standards for HRV calculation. Variations in methods include different durations of epochs, whether re-sampling is used, and whether sleep state was taken into account. Another possible reason for conflicting results is that the significance and meaning of many HRV measures are too complex to be fully understood, so it is possible for incorrect conclusions and for excessive or unfounded extrapolations.

In order to reach a more dependable result in this study, the same standard for all the affected factors are chosen and used based on previous studies. A study of the relationship between these HRV parameters might provide some insight into the slight difference between these HRV measurements, which might help solve the conflicting results in previous studies. 


\section{CHAPTER III}

\section{MEASUREMENT OF HEART RATE VARIABILITY}

Analyzing long-term recordings of physiological signals has been used in several biomedical research areas, especially in the study of sleep disorders and cardiorespiratory abnormalities. Such long-term recordings can be up to 24 hours. In this study, we use the long-term recordings (around 8 hours) of RR interval data to investigate the difference in cardiac signals in infants. As a member of the Collaborative Home Infant Monitoring Evaluation (CHIME) study group, we have access to a polysomnographic (PSG) signal database, which contains approximately 8-hours of PSG data from 529 infants. The PSG data were recorded on a Healthdyne ALICE3 system, which contains electroencephalogram (EEG), electromyogram (EMG), electrocardiogram (ECG), RR interval, actimeter (ACT), and left and right electrooculograms (EOG), and on the CHIME home monitor which contains electrocardiogram (ECG), RR intervals, respiration through inductance plethysmography, pulse oximetry, accelerometer [NEU01, CRO97, NEU00]. For our study, we use the continuous 8-hour RR intervals from the CHIME monitor sampled at $1000 \mathrm{~Hz}$.

In addition, there are also sleep state files for each infant that classify every 30second into four sleep-waking states, quiet sleep (QS), rapid eye movement sleep (REM), awake (AWK), and indeterminate state. This sleep state classification was performed by trained technicians in the CHIME study group based on EEG, EOG, respiratory regularity, and body movements of the infants and smoothed using a special algorithm [CRO97, KUL00]. Since heart rate and heart rate variability (HRV) is highly dependent 
on sleep state, all the analysis in this study is done in different sleep states (QS, REM, and AWK). We will ignore the indeterminate sleep state when considering the analysis, because these periods are often too noisy to be used due to the movement of the infant. Since the indeterminate periods are often short and infrequent, little information is lost by excluding them.

Infants were classified into four different groups upon entrance into the study: healthy, preterm, SIDS siblings, and ALTE (infants with apparent life-threatening events). After a PSG was performed, these infants were outfitted with a specially designed home monitor which recorded their physiological signals as well as lifethreatening events including bradycardia and apnea whenever the monitor was used [NEU01]. Based on the recorded events of each infant which were overread by physicians, a classification was made regarding which infants went on to have future apparent life-threatening events (FALTE) [NEU01, RAM01, HOP96, CRO98]. These data will allow us to study the ability to predict the infant who will go on to have a lifethreatening event through the use of the PSG and derived HRV parameters.

The number of infants that can be included in this study is limited by several restrictions. Although there are 1079 infants enrolled into the CHIME study, the polysomnogram (PSG) study was only performed on 529 of them. Sleep state classification files are available for 380 of these infants. Furthermore, there are also some infants whose data are too noisy to be included. Since we use the RR interval data from the PSG and did all further analysis in different sleep states, we require that the infant must have at least five 5-min segments in each of the three sleep states. Therefore, the number of infants that can be used in our study is limited to 270 . In addition, the RR 
interval files are saved in two types of data formats. We only have the information needed from one data format to extract the RR intervals, which eliminates 131 infants from our study. Lastly, we removed another 17 infants, because they did not use the home monitors for at least 200 hours. Monitor use is important in order to provide enough information to classify infants regarding apparent life-threatening events. Satisfying all the above restrictions, the total number of infants that are included in this study is 122. The RR interval is calculated for these 122 infants, and the infant classification study is based on the data of these 122 infants.

In order to achieve a reliable analysis of the RR interval data, the first step in our research is to locate and correct artifacts in the RR interval data automatically, which is described in chapter 4. The next step is to calculate the heart rate variability (HRV) for the entire eight-hour period using all 5-minute epochs in one continuous sleep state. In most previous studies of $\mathrm{HRV}$, different methods were used as the measure of HRV. In the study for this dissertation, the majority of the most popular methods to measure HRV is used and compared. Lastly, we will evaluate the data in terms of statistical differences between normal infants and infants who went on to have events and to investigate ways to predict those infants.

Many types of parameters have been used to measure heart rate variability, including time domain, frequency domain, time-frequency domain, and nonlinear methods. 


\subsection{Time - Domain (descriptive statistics)}

Time domain analysis, which is the statistical analysis of the fluctuations in RR intervals, is the most commonly used method in the study of HRV. Generally speaking, time domain measures are simple and inexpensive to compute. However, simple descriptive statistics only provide global estimates of HRV. They can not differentiate frequency components and attribute different components to different physiological activities precisely.

In the analysis of $\mathrm{HRV}$, there are two different kinds of variance that emphasize short-term (fast) and long-term (slow) variability. Short-term (beat-to-beat) HRV is the variability calculated based on $\Delta \mathrm{RR}$ (absolute difference between successive $\mathrm{RR}$ intervals). Such measurements reflect short-term HRV measured over a much longer period of time. It is assumed to be associated with parasympathetic (vagal) activity. Long-term (overall) $\mathrm{HRV}$ is the variability measured based on the RR and is assumed to

reflect both sympathetic and vagal influences. There is more disagreement on interpreting long-term variability. It has not been demonstrated whether they are related mainly to sympathetic influences, or to the sympathetic and vagal balance [POR92, MAL95, IZA91]. The parameters used to measure these two kinds of HRV are listed as follows.

- Overall HRV parameters (variability based on RR):

- SDNN: Standard deviation of normal-to-normal RR intervals over a period of time is a broad-band measure, containing both high and low frequency components of RR interval fluctuations [BER97]. In many studies, SDNN is calculated over a 24-hour period and thus it measures all sources of heart rate 
variability, short-term high frequency variations as well as the lowest frequency components seen in the 24-hour period, such as circadian rhythms.

$$
S D N N=\sqrt{\frac{1}{N-1} \sum_{i=1}^{N}\left(R R_{i}-R R_{\text {avg }}\right)^{2}}
$$

- IQRNN: Interquartile range of normal-to-normal RR intervals over a period of time is the difference between the upper and lower 25 percentile of the RR interval time series [LEI80, HAD80].

- NIQRNN: Normalized interquartile range of normal-to-normal RR intervals, is defined as:

$$
N I Q R N N=\frac{\operatorname{IQR}(R R)}{\operatorname{Median}(R R)}
$$

- CV: Coefficient of variation of RR [ANT90],

$$
\begin{gathered}
C V=100 \times \frac{R M S M}{\operatorname{Mean}(R R)} \\
R M S M=\sqrt{\frac{1}{N} \sum_{i=1}^{N}\left(R R_{i}-R R_{\text {avg }}\right)^{2}}
\end{gathered}
$$

RMSM stands for: root mean square difference from the mean

- SDNNIDX: The mean of the standard deviation of successive 5-min RR intervals over a long period of time measures the variability of cycles shorter than 5 minutes [MYE86].

- SDANN: Standard deviation of the means of successive 5-min RR intervals over a long period of time is a longer-term variability, due to cycles longer than 5 minutes [MYE86, BER97]. 
Both SDNNIDX and SDANN are calculated for separate sleep states over the eight hours period in this study.

- Beat-to-beat HRV parameters (variability based on $\Delta \mathrm{RR}$ ):

- SDSD: Standard deviation of successive differences between normal-to-normal RR intervals is defined as:

$$
S D S D=\sqrt{\frac{1}{N-1} \sum_{i=1}^{N}\left(\Delta R R_{i}-\Delta R R_{a v g}\right)^{2}}
$$

○ IQRSD: Interquartile range of successive differences between normal-to-normal RR intervals is the difference between the upper and lower 25 percentile of the $\Delta \mathrm{RR}[\mathrm{LEI} 80, \mathrm{HAD} 80]$.

○ NIQRSD: Normalized interquartile range of successive differences between normal-to-normal RR intervals, is defined as:

$$
N I Q R S D=\frac{\operatorname{IQR}(\Delta R R)}{\operatorname{Median}(R R)}
$$

- RMSSD: Root mean square of successive differences between normal-to-normal RR intervals [ANT90, BIG89, BER97], is defined as

$$
R M S S D=\sqrt{\frac{1}{N-1} \sum_{i=1}^{N-1}\left(R R_{i}-R R_{i+1}\right)^{2}}
$$

○ CVS: Coefficient of variation of $\Delta \mathrm{RR}$ is defined as:

$$
C V S=100 \times \frac{R M S S D}{\operatorname{Mean}(R R)}
$$

$\circ$ pNN50 is defined as the percent of absolute differences between successive normal RR intervals that are greater than $50 \mathrm{~ms}$ [BIG89].

- Counts is defined as the absolute number of differences between successive normal RR intervals that are greater than $50 \mathrm{~ms}$. 


\subsection{Frequency - Domain}

Compared with time domain measures, frequency domain measurements of HRV have the advantage of better separation of parasympathetic and sympathetic influences. Frequency domain methods decompose the total variation of the RR interval time series into many different frequency components, which can be attributed to different physiologic effects. There are three major frequency bands for HRV in adults. The definition of these three frequency bands is not exactly the same throughout the literature. The following are the most commonly used definitions: (1) the high frequency band (about $0.15-0.4 \mathrm{~Hz}$, in normal free-breathing adult), is associated with respiratory sinus arrhythmia (RSA), and has been attributed to parasympathetic (vagal) activity. Its peak frequency varies with respiration. (2) The mid-frequency band (about $0.05-0.15 \mathrm{~Hz}$ ), centered at about $0.1 \mathrm{~Hz}$, is thought to be associated with oscillations of the baroreflex system; (3) the low frequency band $(0.02-0.05 \mathrm{~Hz})$, is attributed to the influences of the vasomotor and rennin-angiotensin control system [MYE86, ORI92, COS94, LKE91, BIG92]. The mid-frequency and low frequency bands are assumed to be under the influence of both parasympathetic and sympathetic activity, but predominantly by the latter. Most investigators combine the mid-frequency and low frequency band into one low frequency component, and distinguish only HF and LF bands.

High frequency power (HF), low frequency power (LF), total power, and LF/HF ratio are the most commonly used parameters in SIDS research. Studies indicate that the physiological origin of the HF components is better understood than that of the LF components [ORI92]. Most researchers agree that vagal activity is the major contributor to the HF component. More controversy was shown in the interpretation of the LF 
component. Some studies suggest that LF is a quantitative marker of sympathetic modulations, while others view LF as reflecting both sympathetic activity and vagal activity [ROT90, YER98]. Consequently, the LF/HF ratio is considered by some investigators to reflect the balance between sympathetic and vagal or to reflect the sympathetic modulation [TASK96]. In the frequency analysis of HRV for infants, the LF band is defined as $0.02-0.2 \mathrm{~Hz}$ or $0.02-0.15 \mathrm{~Hz}$, while the $\mathrm{HF}$ band was $0.2-1.5 \mathrm{~Hz}$ or $0.15-1.5 \mathrm{~Hz}$ in [MRO96] and [MAZ98], respectively.

The most commonly used frequency domain parameters which are used in this thesis are listed:

- Total power is defined as the area under the power spectral curve [ORI92, BIG89, BIG92], or the sum of HF and LF [KLE91]. The latter definition is used in calculating the total power for this study.

O HF: Absolute high frequency power is the area under the power spectral curve related to high frequency band $(0.2-1.5 \mathrm{~Hz}$ for infants). The unit for absolute power is second ${ }^{2}$.

O LF: Absolute low frequency power is the area under the power spectral curve related to low frequency band $(0.02-0.2 \mathrm{~Hz}$ for infants $)$.

○ NHF: Normalized HF is defined as

$$
\frac{H F}{H F+L F}
$$

NLF: Normalized LF is defined as

$$
\frac{L F}{H F+L F}
$$

○ $\mathrm{LF} / \mathrm{HF}$ is defined as the ratio of LF over HF. 
The power spectrum distribution is often assessed by fast Fourier transform (FFT) techniques or autoregressive (AR) modeling. The spectrum curve from AR model is smoother than that from FFT. The results from these two methods are comparable, and we choose to use FFT.

In order to achieve meaningful spectral analysis of $\mathrm{HRV}$, it is necessary to define the data preprocessing procedure. The following aspects must be considered:

- Re-sampling the RR interval time series

Since the RR interval time series signal is sampled unevenly (according to the duration of each beat), the data must be evenly re-sampled. Selecting the optimal sampling frequency is an important step. To avoid aliasing, the required sampling frequency must be at least twice the highest resolvable frequency, and a sampling frequency of 4 times the target frequency is more appropriate. So, for the HRV studies of humans, a sampling rate of $4 \mathrm{~Hz}$ is sufficient to capture the $\mathrm{HF}$ components. That sampling frequency is also appropriate for infants, which have high respiratory rates and adults during exercise [BER97]. We use $4 \mathrm{~Hz}$ sampling frequency to re-sample the RR interval time series in this research.

- Stationarity of the RR interval time series

This concern is not just limited to frequency domain analyses of HRV. Any estimation that attempts to characterize the specific periodicities over time may be distorted by slow linear or irregular trends, and might lead to misinterpretations of the results. Spectral analysis requires that the data series is at least weakly stationary. Actually, in the study of HRV, this is a difficult issue because the actual heart period data are commonly nonstationary. Several methods have been suggested to deal with this 
problem: (a) choosing shorter analytical epochs (2-5min), because nonstationarity increases with the length of the sample epoch; (b) removing slow nonstationary trends before analysis; (c) applying bandpass filters to isolate the frequency component of the interest [BER97]. Otherwise, techniques designed for evaluating nonstationary signals, such as wavelets, should be used. We decided to use the above suggestions (a) and (b) to solve the nonstationarity problem in our data, choosing a proper epoch length and detrending the data. Five minutes will be used for all the HRV analysis, and is further described later. For each 5-min segment RR interval, subtracting the mean of the RR interval in the time domain was used as detrending before spectral analysis.

\subsection{Time - Frequency - Domain}

Power spectral density (PSD) estimation allows quantitative analysis of the frequency components of the RR interval time series, and it is an important method to understand tonic cardiovascular activities. The power spectrum is often assessed by FFT and AR modeling as described above. However, these traditional methods have their own limitation when used in the analysis of heart rate variability. They assume that the signal is at least quasi-stationarity, which is not always true in physiological signals. This obscures the accuracy of PSD estimation of nonstationary signals. Furthermore, traditional spectral analysis methods have an excellent frequency resolution, but their time resolution is insufficient. The wavelet transform, or time-frequency domain methods, is a better alternative. 


\subsubsection{Concept of wavelet transform}

Wavelets have challenged the traditional Fourier transforms for analyzing and processing signals. Instead of relying on fixed sinusoidal functions in Fourier transform, wavelets use a 'mother' wavelet with various shapes. It is a mathematical function that breaks down the signals into a number of scales related to frequency components and analyzes each scale with a certain resolution. This breaking down process is called a wavelet transform. It offers a flexible multiresolution signal representation which is useful in many applications.

In mathematical terms, the continuous wavelet transform (CWT) decomposes a signal $\mathrm{f}(\mathrm{t})$ by performing scalar product with a family of $\Psi(\mathrm{a}, \mathrm{b})$ functions, which are built by dilation and translocation of the mother wavelet $\Psi$. The transform is defined as [ALD96]:

$$
\begin{gathered}
\mathrm{W}_{\mathrm{f}}(\mathrm{a}, \mathrm{b})=\langle\mathrm{f}(\mathrm{t}) \Psi(\mathrm{a}, \mathrm{b})\rangle=\int_{-\infty}^{+\infty} \mathrm{f}(\mathrm{t}) \Psi_{\mathrm{a}, \mathrm{b}}(\mathrm{t}) \mathrm{dt} \\
\text { where } \Psi_{\mathrm{a}, \mathrm{b}}(\mathrm{t})=\frac{1}{\sqrt{a}} \Psi\left(\frac{t-b}{a}\right) \quad \mathrm{a} \neq 0
\end{gathered}
$$

The resultant $\mathrm{W}_{\mathrm{f}}(\mathrm{a}, \mathrm{b})$ are called wavelet coefficients, which represent the correlation between the signal and the chosen wavelet at different scales. The amplitude of the coefficients therefore tends to be maximum at those scales and locations where the signal most resembles the chosen wavelet. The wavelet transform is a tool for timefrequency signal analysis since each wavelet function $\Psi(\mathrm{a}, \mathrm{b})$ is predominantly localized in a certain region of the time-frequency plane. The wavelet function $\Psi(a, b)$ depends on two parameters, scale a and translocation parameter b, which vary continuously over the real numbers. For smaller values of scale a, the wavelet is contracted in the time domain 
and the wavelet transform gives information about the detail of the signal, which is also called high frequency component. For larger values of a, the wavelet expands to give a global view of the signal, which is the low frequency component of the signal.

To reduce the redundancy of the continuous wavelet transform, the discrete wavelet transform (DWT) has been introduced. It can be understood as a CWT sampled on a discrete plane. The most popularly used algorithm in HRV study is the orthogonal multiresolution pyramidal DWT algorithm [MAL892], because of its non-redundancy and computational efficiency. The principle of the dyadic pyramid algorithm is depicted in figure 3.1. This multiresolution pyramidal DWT algorithm is used to analyze the RR interval data for this study.

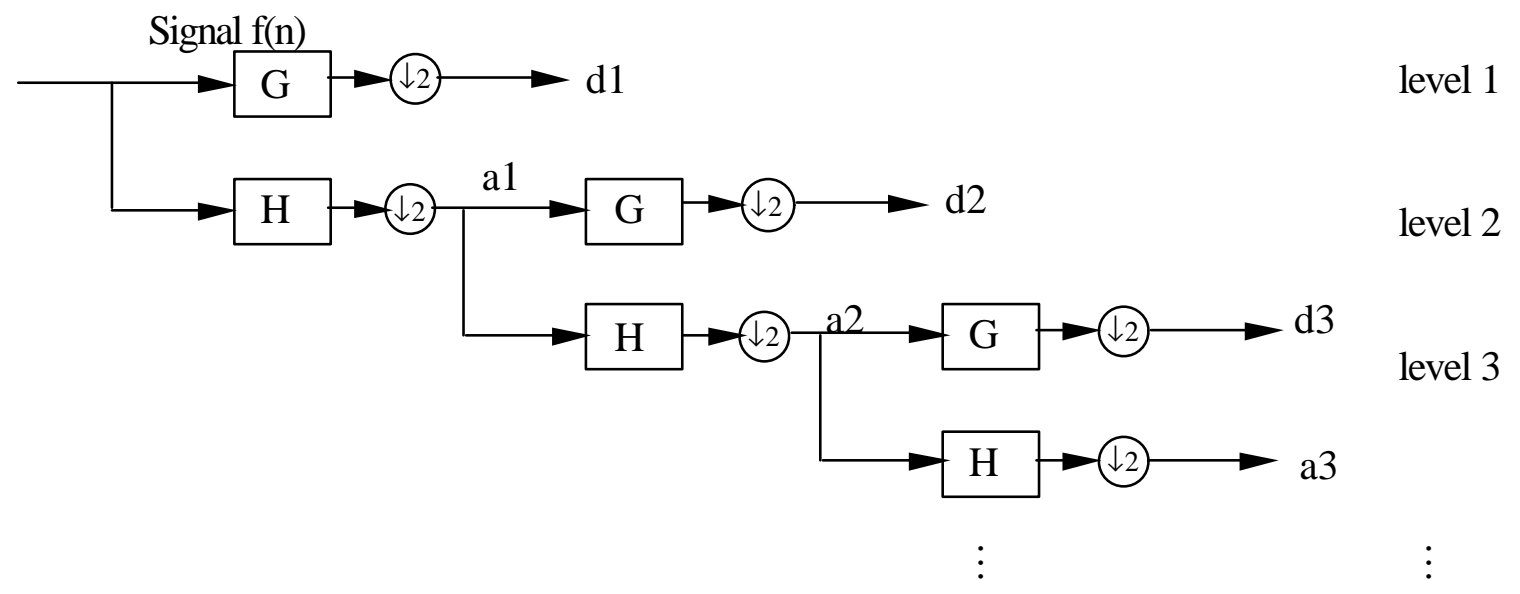

Figure 3.1 Multiresolution pyramidal DWT algorithm

One other important issue is to find out the relationship between wavelet scale (level) and the Fourier frequency, so as to make the results fom both frequency and timefrequency analysis of HRV comparable. The frequency interpretation was given in [MED97] as following: for a given signal sampled at Fs $\mathrm{Hz}$, the wavelet decomposition at 
scale $\mathrm{j}$ acts as a bandpass filter between $\mathrm{Fs} 2^{-\mathrm{j}-1} \mathrm{~Hz}$ and $\mathrm{Fs} 2^{-\mathrm{j}} \mathrm{Hz}$. Previously, this equation has been used to interpret HRV with wavelet in adults [SHI99]. In this dissertation, we used a similar method to define wavelet coefficients that are consistent with frequency definition for infants.

\subsubsection{Time-frequency measurements of heart rate variability}

Like frequency domain measures of HRV, similar parameters are defined here to quantify different frequency components of HRV, which are calculated from the wavelet transform [MED97, SHI99].

O HFW: The high frequency power from the wavelet transform is the sum of the wavelet power coefficients at level 2, 3 and 4 across the entire 5 minutes segment.

○ LFW: The low frequency power from the wavelet transform is the sum of the wavelet power coefficients at level 5, 6 and 7 across the entire 5 minutes segment.

- LFW/HFW is $\mathrm{LF} / \mathrm{HF}$ ratio from the wavelet transform.

Consequently, the above definition of LFW and HFW correspond to the frequency component of 0.1875 to $1.5 \mathrm{~Hz}$ for $\mathrm{HF}$, and 0.0234 to $0.1875 \mathrm{~Hz}$ for $\mathrm{LF}$, appropriate for infants. The time-frequency analysis of HRV using wavelet transform also requires the RR interval to be evenly sampled. According to the relationship between wavelet scale and Fourier frequency and frequency band definition of HF and LF for infants, six Hertz sampling frequency is a proper sampling frequency.

Choosing an appropriate mother wavelet is another important issue in wavelet application. Daubechies 4 wavelet is the most commonly used mother wavelet for the studies of heart rate variability [SHI99, PIC99, BAK95], and was demonstrated as a 
proper mother wavelet for RR interval signal. We chose Daubechies 4 as mother wavelet in our study.

Compared with traditional frequency analysis methods (FFT or AR modeling), the wavelet transform has many advantages. (1) It has no prerequisite over the stationarity of the analyzed signal. (2) The time-frequency localization property of the wavelet transform makes it more suitable for the analysis of nonstationary signals, such as the RR interval. It provides the status of heart rate variability at any time, and can capture any sudden changes in autonomic tone. (2) It exhibits good frequency resolution as well as time resolution. (3) It offers a flexible multiresolution signal representation and variable scale. Long windows can be used to analyze low frequency components, while short windows can be used for high frequency components. (4) It provides different shapes of mother wavelets to fit the analyzed signal better than the fixed sinusoidal shape of the Fourier transform [PIC99].

The most important characteristic of wavelet transform is the multiscale representation and analysis of signals, and time-frequency localization. These properties make it especially suitable for the analysis of nonstationary signals. Most biomedical signals are not stationary and typically have highly complex time-frequency characteristics. Any appropriate analysis method should therefore exhibit good frequency resolution along with fine time resolution.

\subsection{Nonlinear Parameters}

Nonlinear characteristics are certainly involved in HRV. Some investigators emphasize the importance of using nonlinear techniques to quantify HRV. It has been 
suggested that through nonlinear analysis of $\mathrm{HRV}$, it might be possible to obtain precious information for physiological interpretation of $\mathrm{HRV}$ and to predict patients for high risk of sudden cardiac death. However, these nonlinear methods have not been used systematically to investigate large patient populations. At present, they are just potential tools for HRV assessment [TASK96].

\subsubsection{Approximate Entropy (ApEn)}

Approximate Entropy quantifies the regularity in time series data. It has been used in the analysis of the RR interval, and provides information about both beat-to-beat and long-term changes in heart rate.

The definition of ApEn is as follows. For a given time series $\mathrm{x}(1), \mathrm{x}(2) \ldots \ldots$ $\mathrm{x}(\mathrm{N})$, and two fixed parameters $\mathrm{m}$ (positive integer) and $\mathrm{r}$ (positive real), vector sequences $\mathbf{x}(1)$ through $\mathbf{x}(\mathrm{N}-\mathrm{m}+1)$ are formed, where $\mathbf{x}(\mathrm{i})=[\mathrm{x}(1), \ldots . . \mathrm{x}(\mathrm{I}+\mathrm{m}-1)]$, and $\mathrm{m}$ is the length of compared runs. A distance $\mathrm{d}[\mathbf{x}(\mathrm{i}), \mathbf{x}(\mathrm{j})]$ is defined as the maximum difference in their respective scalar components. $C_{i}^{m}$ measures the regularity of patterns similar to a given pattern at compared window length $\mathrm{m}$ and threshold $\mathrm{r}$. Approximate entropy is defined by the following equations.

$$
\begin{gathered}
C_{i}^{m}(r)=\frac{\text { number_of_t }(j) \_t h a t_{-} d[\mathbf{x}(i), \mathbf{x}(j)] \leq r}{N-m+1} \\
\phi^{m}(r)=\frac{1}{N-m+1} \sum_{i=1}^{N-m+1} \ln \left(C_{i}^{m}(r)\right) \\
\text { Then } \quad A p E n(m, r)=\lim _{N \rightarrow \infty} \phi^{m+1}(r)-\phi^{m}(r)
\end{gathered}
$$


ApEn measures the regularity of the signal; increased ApEn value stands for a more irregular signal. To calculate ApEn in $\mathrm{RR}$ interval data, it is recommended to choose 2 and 0.15 times standard deviation of the signal, respectively, for the two fixed parameters window length $\mathrm{m}$ and threshold $\mathrm{r}$. There are some other issues which should be considered for ApEn calculation.

- Re-sampling the RR interval time series

Calculation of the ApEn does not require the RR interval time series to be evenly sampled. However, the number of data points will affect the result of ApEn. It is more meaningful to compare the ApEn calculated from the same number of RR interval data points. Also, it is recommended to calculate ApEn of RR interval time series, using approximately 1000 data points in each segment to gain good results. We decided to resample the RR interval data at $4 \mathrm{~Hz}$ using cubic spline interpolation. Consequently, 5minute segments will result in 1200 sampled RR interval points.

\section{- Detrending}

Whether detrending is necessary while calculating ApEn is also an important consideration. Yeragani et al. performed ApEn and fractal dimension analysis on both detrended and non-detrended RR interval data, and found no significant difference in the result. They explained that linear detrending affects mainly the LF component of HRV. Since the values of ApEn and fractal dimension are correlated significantly with $\mathrm{HF}$ power, the ApEn and fractal dimension values were not affected by the detrending procedure [YER98]. We use the non-detrended RR interval to calculate ApEn for our project. 


\subsubsection{Poincare Plot}

The Poincare plot is a graphical approach that plots the value of a given RR interval (on $\mathrm{x}$ axis) against the subsequent $\mathrm{RR}$ interval (on $\mathrm{y}$ axis). The overall shape of the distribution is used to characterize the dynamics of the time series [BER97]. Figure 3.2 is the Poincare plot of RR intervals from 5 minutes of infant 30166.

The following equations are used to quantify the 2-D Poincare plot [LAN96]:

$$
\begin{gathered}
\sigma_{x}{ }^{2}=\frac{\sum_{i=1}^{N} \sum_{j=1}^{N}\left(x_{i}-\bar{x}\right)^{2} P\left(x_{i}, y_{j}\right)}{\sum_{i=1}^{N} \sum_{j=1}^{N} P\left(x_{i}, y_{j}\right)} \\
\sigma_{y}{ }^{2}=\frac{\sum_{i=1}^{N} \sum_{j=1}^{N}\left(y_{i}-\bar{y}\right)^{2} P\left(x_{i}, y_{j}\right)}{\sum_{i=1}^{N} \sum_{j=1}^{N} P\left(x_{i}, y_{j}\right)} \\
\sigma=\sqrt{\sigma_{x}{ }^{2}+\sigma_{y}{ }^{2}}
\end{gathered}
$$

where $\mathrm{N}$ is the total length of the RR intervals and $\mathrm{P}(\mathrm{x}, \mathrm{y})$ is the two-dimensional histogram of the RR interval. $\sigma_{x}{ }^{2}$ and $\sigma_{y}{ }^{2}$ represent the amount of scatter or dispersion along $\mathrm{x}$ and $\mathrm{y}$ axis respectively. 

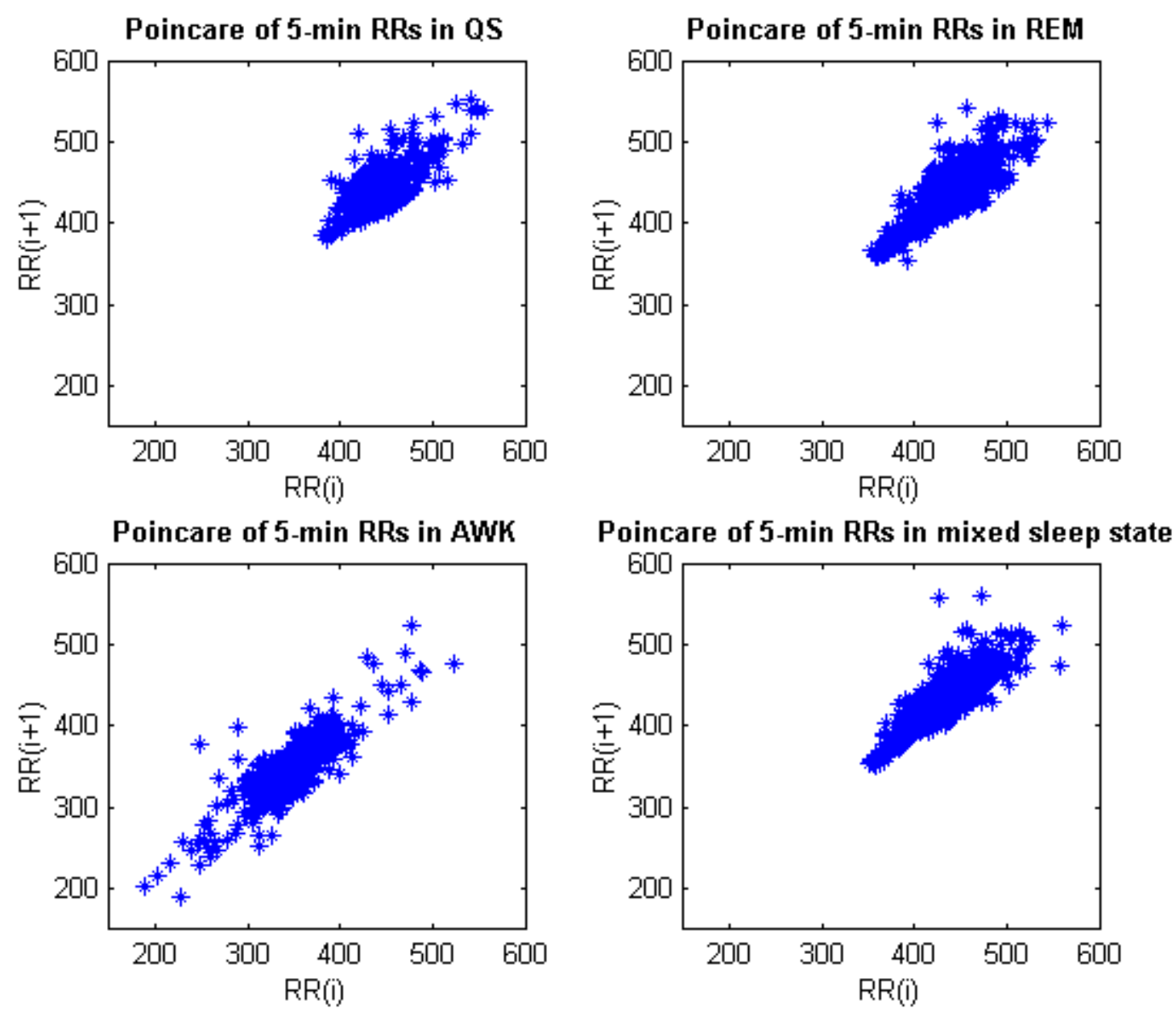

Figure 3.2 Poincare plot of 5-min RR intervals of infant 30166

\subsubsection{Fractal Dimension}

Fractal dimension (FD) is a parameter that measures the space-filling propensity and complexity of the time series. The definition of fractal dimension is given in [KAT88]. Katz defined the fractal dimension of a planar curve as:

$$
D=\frac{\log (L)}{\log (d)}
$$

where $\mathrm{L}$ is the total length of the curve, which can be calculated as the sum of the distances between successive points, and $\mathrm{d}$ is the diameter of the curve, which is defined 
as the longest distance between the first point and any other points. As recommended by Katz, the formula for FD computation becomes:

$$
D=\frac{\log \left(\frac{L}{a}\right)}{\log \left(\frac{d}{a}\right)}=\frac{\log (n)}{\log (n)+\log \left(\frac{d}{L}\right)}
$$

where $\mathrm{a}$ is the average distance between successive points, and $\mathrm{n}=\mathrm{L} / \mathrm{a}$, which equals the total number of points minus one.

Before calculating the fractal dimension, a natural logarithmic transformation was taken on the RR interval time series as described in [YER98].

\subsection{Considerations in HRV Measurements}

As described in chapter 2, different standards for HRV calculation might result in different or even conflicting result in HRV analysis. We used the same standard for the HRV calculation of all infants. Determination of the standard for HRV calculation is also an important step to ensure more comparable results. This section discusses the factors that will affect HRV analysis, and define the standard used for our study based on previous research.

\subsubsection{Selection of appropriate analytical epochs}

HRV in both time and frequency domain are greatly influenced by the length of epochs being analyzed. It tends to increase with the length of the epochs analyzed. It is not meaningful to compare HRV measures, especially overall HRV, obtained from recordings of different duration. Comparison must be done for a fixed length of data. It is 
necessary to define criteria for choosing the duration. Shorter epochs are recommended, because RR intervals over longer periods of time are more likely to be nonstationary, and HRV calculated from them are influenced by slower trends. Moreover, the slower heart rate activity is not clearly vagal and reflects interactions with the sympathetic nervous system. Its removal reduces the non-vagal influences on these HRV measures. Shorter epochs function as filtering the slow heart rate activity, and make the HRV measures more focused on the vagal influences [IZA91].

Stationarity of the data is an important issue to be considered when choosing the length of epochs, especially for the spectral analysis of HRV. Most biomedical signals are not stationary. Physical activity and posture changes can be the cause of nonstationarity. Since many signal analysis techniques require stationarity from the signal, there is no ideal solution to this problem. Choosing shorter segments may somewhat solve this problem, because during short duration, the subject's posture, activity and respiratory frequency can be considered a constant. The use of short segments and a Hanning window in the time domain (which further shortens the 'effective' length of a segment) also tend to minimize the effects of nonstationarity [MYE86]. However, the epochs cannot be too short, because biological rhythms generally vary from cycle to cycle. Recordings should be sufficiently long to evaluate the slow variance of interest. The recording duration should be at least 10 times the wavelength of the lower frequency component that was investigated. For the HRV analysis, a recording of approximately one minute is needed to address the HF component, and approximately two minutes is needed to assess LF component. Five minutes segments are highly recommended for clinical studies of HF and LF variability. 
Analyzing the HRV power spectrum over a long periods of time by computing the power spectral density of consecutive 5-min segments is also highly recommended. There are some studies using the entire 24-hour data for the spectral analysis of HRV. Indeed, the RR interval data cannot be considered stationary during the 24-hour period. Thus, the spectrum obtained from shorter segments (5-min) averaged over the entire 24-hour period is more meaningful, and provides averages of the modulations attributable to the $\mathrm{HF}$ and LF components [TASK96].

A variety of epochs were used in the study of HRV, such as 30-sec [IZA91], 60sec [ROS99, HAR76, SCH98], 120-sec [SPI87, ANT90, MYE86, LIA96], and 100-sec [STE88]. Stevens et al. supports 100-second segments, because it is sufficiently long to get a reliable estimate of parameters, while it is also sufficiently short to allow short-term pattern changes to become apparent. Some researchers use the whole length of the data, 24-hour recording [LEI80, PIK99]. A 5-minute segment is the most popular [ROT90, BIG92, EDL94, BER97, KLE91, TASK96]. We decided to use 5-minute segments for the HRV analysis in our project on the entire 8 hour PSG, as the best balance between stationarity issues and enough data to be representative.

\subsubsection{Overlapped or non-overlapped segments}

Most of the analyses of HRV have been performed for nonoverlapping consecutive segments of the RR interval time series. However overlapped segments were also used in some studies [MYE86, COS94]. Myers et al used overlapped segments of RR interval data in the frequency analysis of HRV. Up to 20 segments of 2 -min epochs of RR intervals were selected in each hour, beginning on the hour. Succeeding segments 
began at the midpoint of the previous segment. Twenty consecutive segments were used if the data in these segments was acceptable. Most of the time they used the first 21 minutes of data each hour. Noisy segments or segments with premature beats were excluded. In such an instance, the segment began three beats after the last premature or noisy beat. When doing frequency analysis, each 2-min epoch of RR intervals were evenly sampled to 1024 points, the mean of the RR interval was subtracted, and a Hanning window was applied in the time domain. Lastly, an FFT was performed to estimate the power spectrum [MYE86]. We use non-overlapped segments for this research. All of the above HRV parameters will be calculated for every 5-min consecutive non-overlapped segments in the same sleep state from the entire 8-hour polysomnographic (PSG) data.

\subsubsection{Effect of sleep state on heart rate variability}

Heart rate and heart rate variability are sleep state dependent variables. It is assumed that the awake state has the highest heart rate and heart rate variability, and quiet sleep has the lowest value of heart rate and variability. The value for REM is in between. Landes et al. found that heart rate is the slowest and contains the highest frequencies in quiet sleep [LAN96]. Figure 3.3 plots he RR intervals of three sleep states for one infant. The difference is apparent. Sleep state differences are also shown in the frequency analysis of HRV. Medigue et al. found that the high frequency component of HRV (HF) dominates during QS, while low frequency component of HRV (LF) dominates in REM [MED97]. Therefore, in our study we perform all the HRV analysis and group comparisons in separate sleep states. 


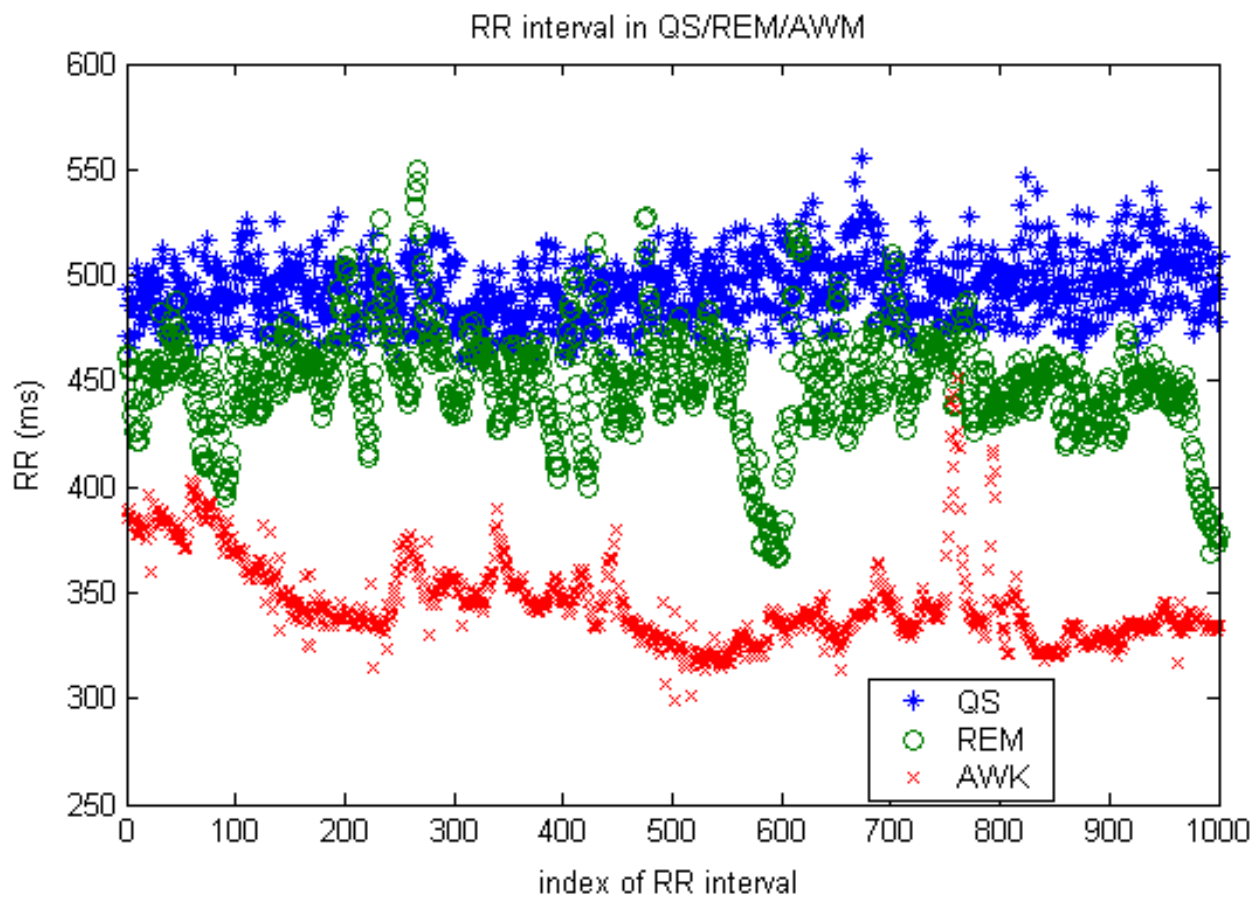

Figure 3.3 RR intervals in three sleep states for one infant

\subsection{Results of HRV Calculation for the Infant Dataset}

In the study for this dissertation, we included the most popular HRV parameters as described above, and formed a fixed standard in HRV calculation and analysis for all infants. There are $24 \mathrm{HRV}$ parameters studied: Mean, Median, SDNN, IQRNN, NIQRNN, CV, SDSD, IQRSD, NIQRSD, RMSSD, CVS, pNN50, HF, LF, NHF, NLF, total power, LF/HF, HFW, LFW, LFW/HFW, ApEn, Poincare, and Fractal Dimension. For the 8-hour RR interval, the HRV parameters are calculated for non-overlapped consecutive 5-min segments with sleep state as a consideration. Resampling was only performed for frequency, time-frequency measurements and approximate entropy. Figure 3.4 shows four HRV parameters versus time for a healthy infant (20138) as an example of the resultant HRV measurements. Each point in the plot indicates the HRV value for a 
5-minute segment. For each sleep state, a vector of HRV values is obtained per infant. All 5-min segments, rather than an averaged value, will be used in further analysis. The average HRV per sleep state per infant is used in most of the previous studies, which might hide the variance. Using the continuous long-term HRV instead of the average values over a long period of time should help capture the difference between infants.

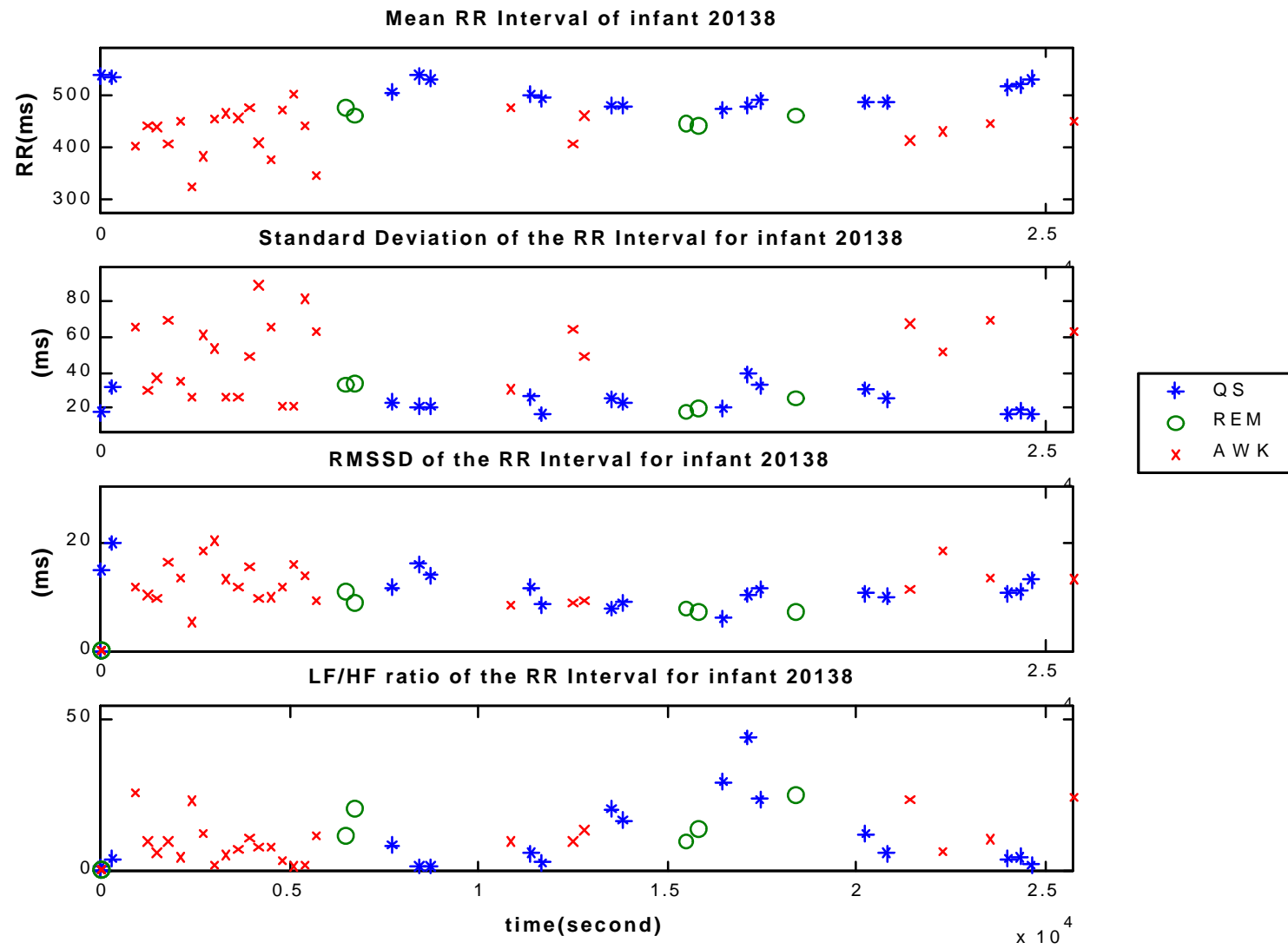

Figure 3.4 Example of four HRV parameters (Mean, Standard Deviation, RMSSD, and $\mathrm{LF} / \mathrm{HF}$ ratio) over an 8-hour period

Each point is the results for a 5-minute interval

Similar results were obtained for the 12 time-domain, 6 frequency-domain, 3 time-frequency-domain, and 3 nonlinear measurements of heart rate variability. These resultant HRV values will be used for studying the relationship between different HRV 
parameters, evaluating the difference between different infant groups statistically, and classifying infants with regard to life-threatening events.

\subsubsection{Statistical summary of infant HRV}

The above HRV parameters are calculated for 122 infants from our CHIME infant dataset. Twenty of them are categorized as event infants. A more detailed explanation is given in Chapter 6. In order to get a general understanding of our infant HRV characteristics, we calculate the average value for HRVs in each sleep state for each infant, and then obtain the mean and standard deviation across infants. Table 3.1 shows the results of the HRV measurement for all of the 122 infants. Figure 3.5 visually represents the changes across sleep state in four selected HRV parameters.

Since the infants in our study are categorized into two groups, healthy infants and infants with future apparent life-threatening events (FALTE), according to the recorded event information on the home monitor, the same statistical summary is also done on these two groups of infants separately. There are 102 infants in the control group, and there are 20 infants in the event group. Table 3.2 shows the results. Group difference is observed in some of the parameters. 
Table 3.1 HRV measurements for all 122 infants

\begin{tabular}{|c|ccc|ccc|ccc|}
\hline HRV & \multicolumn{3}{|c|}{ QS } & \multicolumn{3}{|c|}{ REM } & \multicolumn{3}{c|}{ AWK } \\
\hline mean & 443.87 & \pm & 47.80 & 432.18 & \pm & 40.46 & 374.19 & \pm & 30.41 \\
median & 442.74 & \pm & 47.57 & 432.23 & \pm & 40.37 & 370.79 & \pm & 30.81 \\
SDNN & 17.57 & \pm & 7.34 & 30.13 & \pm & 9.40 & 31.07 & \pm & 7.33 \\
IQRNN & 20.37 & \pm & 9.24 & 36.45 & \pm & 13.22 & 39.96 & \pm & 10.55 \\
NIQRNN & 0.04 & \pm & 0.02 & 0.08 & \pm & 0.03 & 0.11 & \pm & 0.02 \\
CV & 3.86 & \pm & 1.30 & 6.88 & \pm & 1.72 & 8.27 & \pm & 1.72 \\
SDSD & 6.87 & \pm & 3.76 & 8.65 & \pm & 3.58 & 8.23 & \pm & 2.55 \\
IQRSD & 8.41 & \pm & 4.74 & 9.02 & \pm & 4.17 & 7.36 & \pm & 3.21 \\
NIQRSD & 0.02 & \pm & 0.01 & 0.02 & \pm & 0.01 & 0.02 & \pm & 0.01 \\
RMSSD & 10.98 & \pm & 6.16 & 12.29 & \pm & 5.21 & 10.92 & \pm & 3.59 \\
CVS & 2.37 & \pm & 1.11 & 2.78 & \pm & 0.97 & 2.90 & \pm & 0.86 \\
pNN50 & 0.01 & \pm & 0.02 & 0.01 & \pm & 0.02 & 0.01 & \pm & 0.01 \\
\hline HF & 22617.09 & \pm & 28151.86 & 26113.82 & \pm & 23546.72 & 18427.41 & \pm & 13248.59 \\
LF & 61393.19 & \pm & 60804.38 & 195410.96 & \pm 141590.59 & 139239.38 & \pm & 159122.40 \\
NHF & 30.60 & \pm & 13.44 & 12.73 & \pm & 5.25 & 18.69 & \pm & 7.94 \\
NLF & 69.40 & \pm & 13.44 & 87.27 & \pm & 5.25 & 81.31 & \pm & 7.94 \\
totalpower & 84010.27 & \pm & 82203.79 & 221524.78 & \pm & 160680.85 & 157666.79 & \pm & 162667.76 \\
LF/HF & 4.82 & \pm & 4.19 & 10.40 & \pm & 5.53 & 10.78 & \pm & 11.33 \\
HFW & 148446.49 & \pm & 179988.51 & 171324.80 & \pm & 147880.79 & 123371.05 & \pm & 87429.83 \\
LFW & 367706.73 & \pm & 356780.40 & 1012244.90 & \pm & 724728.42 & 710078.95 & \pm & 445480.84 \\
LFW/HFW & 4.13 & \pm & 3.01 & 7.66 & \pm & 3.39 & 7.33 & \pm & 3.68 \\
\hline ApEn & 1.08 & \pm & 0.14 & 0.83 & \pm & 0.14 & 0.74 & \pm & 0.15 \\
Poincare & 16.14 & \pm & 7.58 & 26.38 & \pm & 10.23 & 30.45 & \pm & 8.21 \\
FD & 1.15 & \pm & 0.06 & 1.17 & \pm & 0.05 & 1.18 & \pm & 0.05 \\
\hline
\end{tabular}

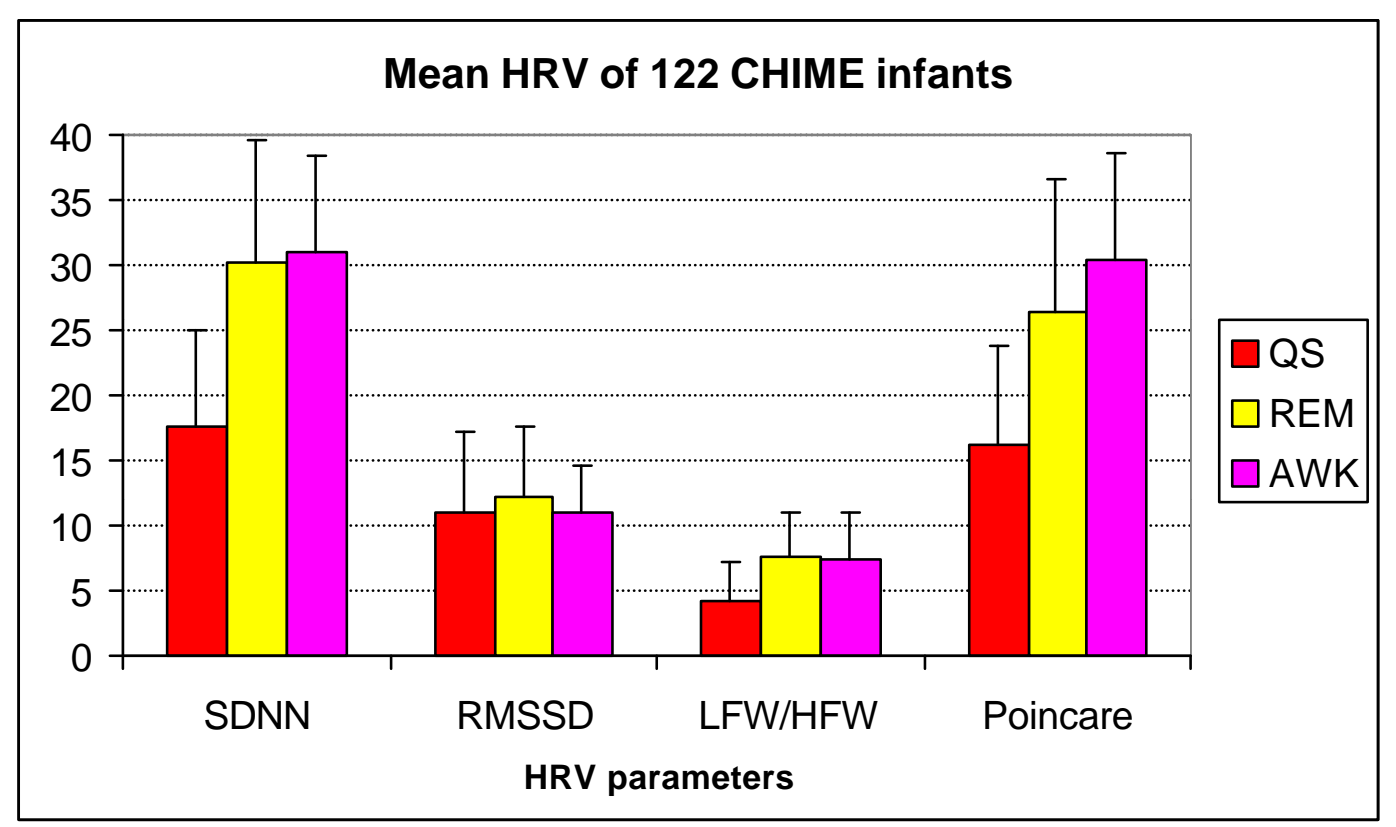

Figure 3.5 Sleep state differenced in HRV of the 122 infants 
Table 3.2 HRV measurements for the two groups of infant

\begin{tabular}{|c|ccc|ccc|}
\hline HRV & \multicolumn{3}{|c|}{ Control Infants } & \multicolumn{3}{|c|}{ Event Infants } \\
\hline mean & 447.69 & \pm & 47.32 & 424.40 & \pm & 46.60 \\
median & 446.67 & \pm & 47.12 & 422.73 & \pm & 45.85 \\
SDNN & 17.68 & \pm & 7.41 & 17.01 & \pm & 7.12 \\
IQRNN & 20.65 & \pm & 9.14 & 18.96 & \pm & 9.85 \\
NIQRNN & 0.04 & \pm & 0.02 & 0.04 & \pm & 0.02 \\
CV & 3.85 & \pm & 1.30 & 3.89 & \pm & 1.32 \\
SDSD & 6.93 & \pm & 3.74 & 6.54 & \pm & 3.95 \\
IQRSD & 8.57 & \pm & 4.71 & 7.60 & \pm & 4.91 \\
NIQRSD & 0.02 & \pm & 0.01 & 0.02 & \pm & 0.01 \\
RMSSD & 11.16 & \pm & 6.15 & 10.04 & \pm & 6.31 \\
CVS & 2.40 & \pm & 1.10 & 2.24 & \pm & 1.17 \\
pNN50 & 0.01 & \pm & 0.02 & 0.01 & \pm & 0.02 \\
\hline HF & 23617.8 & \pm & 29354.4 & 17513.4 & \pm & 20828.8 \\
LF & 61832.1 & \pm & 63086.0 & 59154.9 & \pm & 48814.0 \\
NHF & 31.7 & \pm & 13.4 & 25.0 & \pm & 12.4 \\
NLF & 68.3 & \pm & 13.4 & 75.0 & \pm & 12.4 \\
totalpower & 85449.9 & \pm & 85732.4 & 76668.2 & \pm & 62411.9 \\
LF/HF & 4.7 & \pm & 4.4 & 5.6 & \pm & 2.9 \\
HFW & 154733.6 & \pm & 187821.1 & 116382.3 & \pm & 132258.1 \\
LFW & 369225.7 & \pm & 369197.5 & 359960.3 & \pm & 293252.5 \\
LFW/HFW & 4.0 & \pm & 3.1 & 4.7 & \pm & 2.5 \\
\hline ApEn & 1.08 & \pm & 0.15 & 1.06 & \pm & 0.14 \\
Poincare & 16.34 & \pm & 7.52 & 15.10 & \pm & 8.00 \\
FD & 1.16 & \pm & 0.06 & 1.15 & \pm & 0.06 \\
\hline
\end{tabular}

(a) Quiet Sleep

\begin{tabular}{|c|ccc|ccc|}
\hline HRV & \multicolumn{3}{|c|}{ Control Infants } & \multicolumn{3}{c|}{ Event Infants } \\
\hline mean & 436.09 & \pm & 39.33 & 412.22 & \pm & 41.23 \\
median & 436.31 & \pm & 39.29 & 411.45 & \pm & 40.40 \\
SDNN & 30.21 & \pm & 9.57 & 29.73 & \pm & 8.70 \\
IQRNN & 36.76 & \pm & 13.44 & 34.92 & \pm & 12.27 \\
NIQRNN & 0.08 & \pm & 0.03 & 0.08 & \pm & 0.03 \\
CV & 6.83 & \pm & 1.74 & 7.12 & \pm & 1.65 \\
SDSD & 8.64 & \pm & 3.61 & 8.73 & \pm & 3.51 \\
IQRSD & 9.18 & \pm & 4.20 & 8.24 & \pm & 4.04 \\
NIQRSD & 0.02 & \pm & 0.01 & 0.02 & \pm & 0.01 \\
RMSSD & 12.38 & \pm & 5.26 & 11.83 & \pm & 5.04 \\
CVS & 2.77 & \pm & 0.98 & 2.79 & \pm & 0.95 \\
pNN50 & 0.01 & \pm & 0.02 & 0.01 & \pm & 0.01 \\
\hline HF & 26771.1 & \pm & 24537.5 & 22761.5 & \pm & 17783.2 \\
LF & 199729.4 & \pm & 146752.5 & 173386.7 & \pm & 112162.3 \\
NHF & 12.7 & \pm & 5.0 & 12.9 & \pm & 6.5 \\
NLF & 87.3 & \pm & 5.0 & 87.1 & \pm & 6.5 \\
totalpower & 226500.6 & \pm & 166983.2 & 196148.3 & \pm & 124052.9 \\
LF/HF & 10.4 & \pm & 5.7 & 10.6 & \pm & 5.0 \\
HFW & 173922.8 & \pm & 153369.2 & 158075.0 & \pm & 118362.3 \\
LFW & 1023443.5 & \pm & 748898.8 & 955132.0 & \pm & 599524.7 \\
LFW/HFW & 7.6 & \pm & 3.4 & 7.8 & \pm & 3.2 \\
\hline ApEn & 0.84 & \pm & 0.14 & 0.78 & \pm & 0.14 \\
Poincare & 26.56 & \pm & 10.43 & 25.45 & \pm & 9.32 \\
FD & 1.17 & \pm & 0.05 & 1.17 & \pm & 0.05 \\
\hline
\end{tabular}

(b) Rapid Eye Movement Sleep 
Table 3.2 HRV measurements for the two groups of infant (Cont.)

\begin{tabular}{|c|ccc|ccc|}
\hline HRV & \multicolumn{3}{|c|}{ Control Infants } & \multicolumn{3}{|c|}{ Event Infants } \\
\hline mean & 377.08 & \pm & 28.18 & 359.45 & \pm & 37.36 \\
median & 373.76 & \pm & 28.54 & 355.63 & \pm & 37.76 \\
SDNN & 31.28 & \pm & 7.46 & 30.04 & \pm & 6.71 \\
IQRNN & 40.68 & \pm & 10.56 & 36.24 & \pm & 9.98 \\
NIQRNN & 0.11 & \pm & 0.03 & 0.10 & \pm & 0.02 \\
CV & 8.27 & \pm & 1.77 & 8.29 & \pm & 1.43 \\
SDSD & 8.10 & \pm & 2.34 & 8.88 & \pm & 3.43 \\
IQRSD & 7.45 & \pm & 3.09 & 6.91 & \pm & 3.84 \\
NIQRSD & 0.02 & \pm & 0.01 & 0.02 & \pm & 0.01 \\
RMSSD & 10.85 & \pm & 3.37 & 11.33 & \pm & 4.63 \\
CVS & 2.86 & \pm & 0.81 & 3.11 & \pm & 1.08 \\
pNN50 & 0.01 & \pm & 0.01 & 0.01 & \pm & 0.01 \\
\hline HF & 18393.9 & \pm & 13323.3 & 18598.2 & \pm & 13197.6 \\
LF & 143514.8 & \pm & 171998.0 & 117434.9 & \pm & 58230.9 \\
NHF & 18.4 & \pm & 7.7 & 20.1 & \pm & 9.0 \\
NLF & 81.6 & \pm & 7.7 & 79.9 & \pm & 9.0 \\
totalpower & 161908.7 & \pm & 175670.0 & 136033.1 & \pm & 62284.4 \\
LF/HF & 11.1 & \pm & 12.2 & 9.2 & \pm & 4.8 \\
HFW & 122060.0 & \pm & 88032.3 & 130057.4 & \pm & 86186.0 \\
LFW & 708004.7 & \pm & 453700.8 & 720657.7 & \pm & 411663.6 \\
LFW/HFW & 7.3 & \pm & 3.7 & 7.3 & \pm & 3.8 \\
\hline ApEn & 0.75 & \pm & 0.15 & 0.73 & \pm & 0.18 \\
Poincare & 30.93 & \pm & 8.31 & 28.02 & \pm & 7.36 \\
FD & 1.18 & \pm & 0.05 & 1.18 & \pm & 0.06 \\
\hline
\end{tabular}

(c) Awake

From the above results, there are differences across sleep state and between the two groups in some HRV parameters. However, it is unclear whether these differences are statistically significant. A t-test across infants is applied on the average HRV in each sleep state for each individual infant to evaluate whether there are significant differences in a specific HRV parameter between these two groups, and across sleep states.

\subsubsection{Differences in HRV across sleep states}

A two sample ttest is applied to the mean HRV parameters for three sleep states from the 122 infants to examine the sleep state differences. 
The null hypothesis for this $\mathrm{t}$ test is that the mean HRV from either of the two sleep states are equal to each other. To test the null hypothesis, we calculate the following values: $\bar{x}_{1}, \bar{x}_{2}$ (the means of the two samples), $s_{1}^{2}, s_{2}^{2}$ (the variances of the two samples), $n_{1}, n_{2}$ (the sample sizes of the two samples), $\mathrm{k}$ (the degrees of freedom), and the t value.

$$
t=\frac{\bar{x}_{1}-\bar{x}_{2}}{\sqrt{\frac{s_{1}^{2}}{n_{1}}+\frac{s_{2}^{2}}{n_{2}}}} \text { and } k=\frac{\left(\frac{s_{1}^{2}}{n_{1}}+\frac{s_{2}^{2}}{n_{2}}\right)^{2}}{\frac{\left(s_{1}^{2} / n_{1}\right)^{2}}{n_{1}-1}+\frac{\left(s_{2}^{2} / n_{2}\right)^{2}}{n_{2}-1}}
$$

The degrees of freedom is calculated using the variance of the specific HRV parameters. It varies according to the HRV parameters compared, ranges from 157 to 242. The critical value of the $\mathrm{t}$ distribution at $95 \%$ confidence interval is 1.645 . According to this critical value, statistically significant differences are found in most of the HRV parameters across sleep states. However, there is no difference in SDNN, SDSD, NIQRSD, CVS, SDNNIDX, LF/HF, LFW/HFW, and FD between AWK and REM, also no difference in NIQRSD, RMSSD, pNN50, HF, and HFW between QS and AWK, and no difference in IQRSD, pNN50, SDANN, HF, and HFW between QS and REM. We can conclude that the sleep state differences in most of the HRV parameters are statistically significant.

\subsubsection{Differences in HRV between two infant groups}

Before using heart rate variability to classify infants, we first try to determine if there are significant statistical differences in HRV between FALTE infants and the controls. The same t-test is first applied on the mean HRV parameters of individual 
infants from the two infant groups, the FALTE infant group and the control. The test is performed with the sleep state taken into account. The results are consistent across sleep states. The t-test is performed on $28 \mathrm{HRV}$ parameters. To reach the overall significant level at 0.05 , the significant level for each $\mathrm{t}$-test is 0.0018 . The critical value of the $\mathrm{t}$ distribution at 0.001 is 3.090 . Therefore, at $95 \%$ confidence interval, statistically significant differences are only found in the duration of REM time, and the ratio of REM to the other sleep states between these two groups of infants.

Besides the information of whether there are significant differences between the two groups, the t-value also indicates the mean of which group is larger. A positive tvalue shows the first mean is larger than the second. The HRV for the event infants is expected to be smaller than that of the control infants. The results from our ttest confirm it. However, the duration of REM time for the healthy infants is shorter than that of the event infants. This is in conflict with the results from one previous study, which suggested that the reduced REM sleep might be associated with a high risk of sudden infant death [BAK77].

Since few significant differences were found between the event and control group, it might be difficult to classify infants. Since these results are different from previous studies, which did find significant differences in HRV, we explore the characteristics of our dataset further. Besides the small size of the dataset, the age difference of the two groups is explored in the next section and in Chapter 6. 


\subsubsection{Changes of HRV with post conceptional age in infants}

The heart rate and heart rate variability measurements in this study are performed on the individual infants at different post conceptional ages (PCA). This additional information allows us to investigate whether there is a trend in HRV with the PCA.

Heart rate variability should increase with the maturity of the autonomic nervous system in infants [HAD80, SCH98]. In this section of the dissertation, we study the changes in all of the HRV parameters with PCA. The mean HRV across all 5-min in each sleep state is calculated for 120 infants, because the PCA of two of infants is not available. The PCA of the infants ranges from 34.3 to 62.9 weeks. An example result in Figure 3.6 shows how IQRNN changes with the PCA. The first plot is the average HRV of individual infants versus PCA. To generate the second bar chart, we categorize the 120 infants into 6 age groups at 5-year intervals, the mean HRV across the infants in each group is calculated and used in the bar chart.
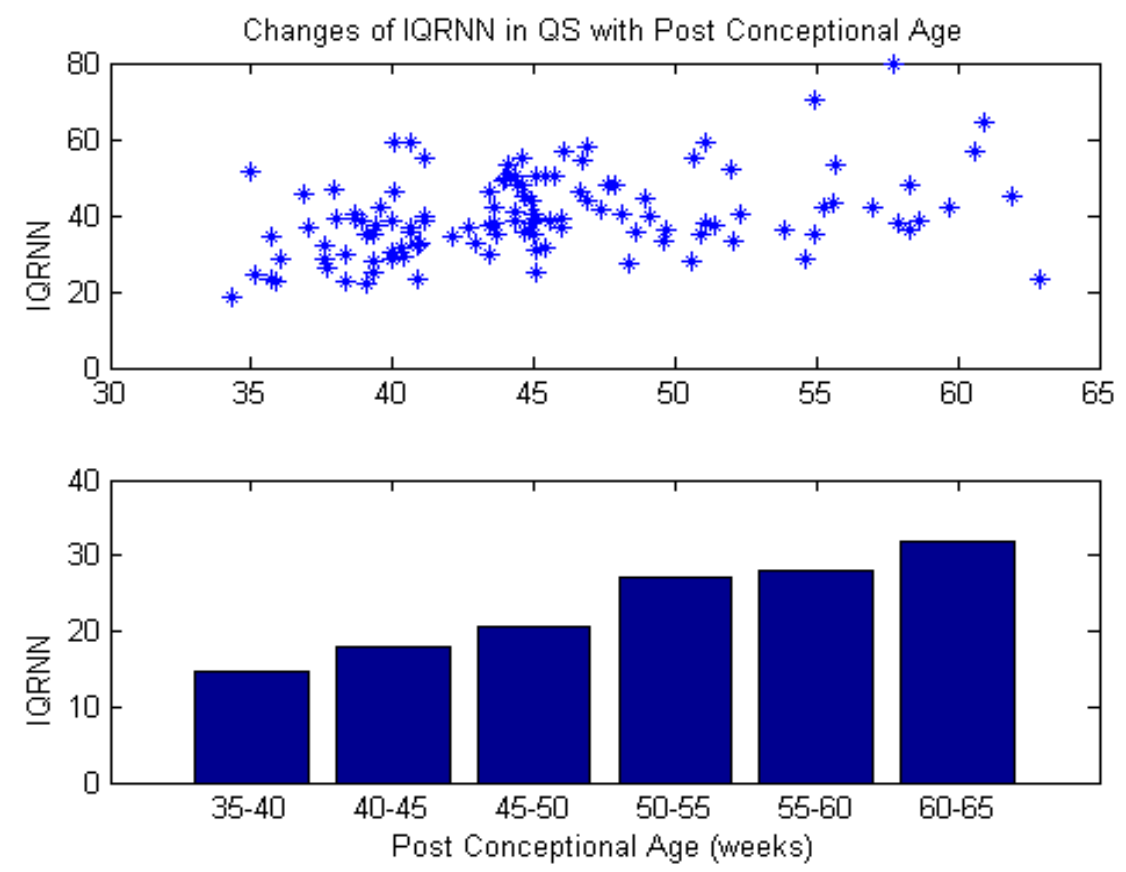

Figure 3.6 Changes of HRV with post conceptional age 
The trend in the interquartile range of RR intervals with PCA is obvious. The HRV increases with increase in PCA. Similar results are reached in most of the other HRV parameters. LF/HF ratio, NHF, NLF, ApEn, FD, REMtime, and REMratio are exceptions, where there is no increasing trend. The changes in HRV in the three sleep states are consistent.

Correlation coefficients between PCA and HRV parameter are also calculated using the average value for each infant per sleep state. The results showed that there are some correlation between the PCA and most of the HRV parameters, except NHF, NLF, $\mathrm{LF} / \mathrm{HF}$, LWF/HFW, and ApEn (Table 3.3). REMratio and REM time is somewhat negatively correlated $(\mathrm{r}=-0.41)$. Slight differences among the three sleep states are also observed.

Table 3.3 Correlation coefficients between PCA and HRV parameters

\begin{tabular}{|c|ccc}
\hline & QS & REM & AWK \\
\hline Mean & 0.7164 & 0.6811 & 0.5053 \\
Median & 0.7171 & 0.6737 & 0.5035 \\
SDNN & 0.5177 & 0.3563 & 0.2902 \\
IQRNN & 0.5400 & 0.3158 & 0.3551 \\
NIQRNN & 0.4492 & 0.1675 & 0.2121 \\
CV & 0.4125 & 0.1902 & 0.1399 \\
SDSD & 0.5381 & 0.4495 & 0.1162 \\
IQRSD & 0.5564 & 0.4878 & 0.2799 \\
NIQRSD & 0.4981 & 0.4098 & 0.1869 \\
RMSSD & 0.5636 & 0.4842 & 0.1980 \\
CVS & 0.5099 & 0.3971 & 0.0705 \\
pNN50 & 0.3885 & 0.4138 & 0.0886 \\
SDNNIDX & 0.5177 & 0.3563 & 0.2902 \\
LF & 0.5365 & 0.5228 & 0.2569 \\
NHF & 0.4275 & 0.3510 & -0.0906 \\
NLF & -0.4275 & -0.3510 & 0.0906 \\
total power & 0.4995 & 0.4114 & 0.0818 \\
LF/HF & -0.2876 & -0.2334 & -0.0759 \\
HFW & 0.5359 & 0.4946 & 0.2651 \\
LFW & 0.4176 & 0.3661 & 0.2091 \\
LFW/HFW & -0.3230 & -0.2429 & -0.0482 \\
ApEn & -0.1659 & 0.1157 & 0.0547 \\
Poincare & 0.5618 & 0.3198 & 0.3389 \\
FD & 0.4744 & 0.3610 & 0.0985 \\
REMtime & -0.4139 & -0.4139 & -0.4139 \\
REMratio & -0.4107 & -0.4107 & -0.4107 \\
\hline
\end{tabular}

These obvious changes in heart rate variability with PCA suggest that it might make it difficult to classify event and control infants, because the change in HRV due to PCA masks the differences between groups. The effect of PCA on the infant 
classification is explored in Chapter 6. Figure 3.7 shows the distribution of PCA among event and control infants. Unfortunately, the distributions of the two groups of infants are not similar. The PCA of the event infants are younger than those of the control infants. The age influence on the HRV will definitely affect construction of the classification model.
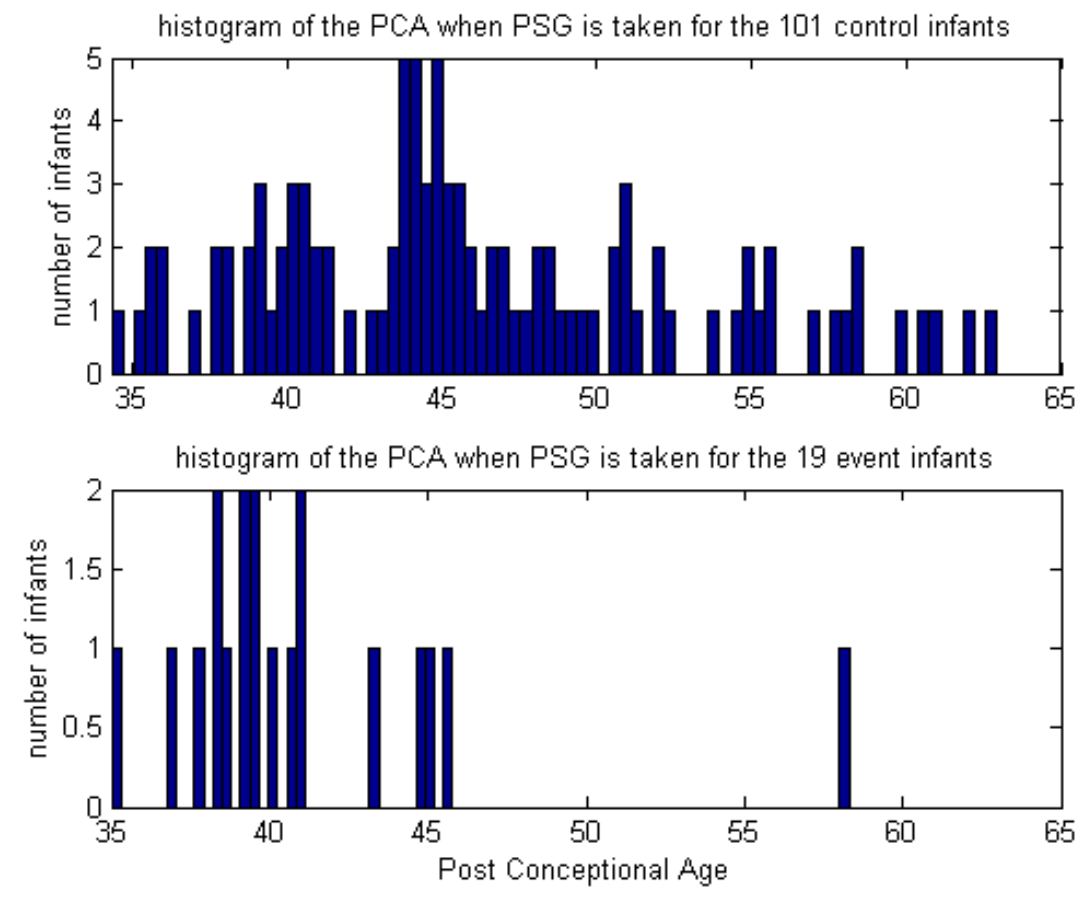

Figure 3.7 Distribution of post conceptional age for event and control infants

\subsection{Summary}

Heart rate variability is a complex and often ambiguous variable. Lack of standardized quantification procedures for $\mathrm{HRV}$ is the most significant barrier for further diagnostic and clinical use of it. Different or even conflicting results of their studies might be because of using different HRV measurements and standards for HRV 
calculation. Such problems include: lack of rules for artifact rejection and correction, choosing re-sampling frequency, choosing length of segment for HRV evaluation, considering sleep states, stationarity of data within a time period as well as standardized methods to interpret different HRV measures. Determination of the affected factors for HRV measures, and further study into exposing the relationship between these measures is important for proper use of HRV. Chapter 3 gives an overview of the methods performed in the thesis to address these issues. Chapter 4 addresses removal of artifact and Chapter 5 is additional work exploring HRV parameters and their relationship. 


\section{CHAPTER IV}

\section{ARTIFACTS IN THE HEART PERIOD DATA}

A major consideration before analyzing heart period data or RR intervals is the removal of artifacts. Continuous electrocardiography (ECG) signals, especially long-term ECG, contain both environmental and biological noise. Heart rate or RR intervals calculated from the ECG inevitably contain artifacts, which may introduce considerable bias in heart rate variability (HRV) measurements and affect the proper use of HRV in clinical diagnosis or research. This chapter discusses previous work and work performed for this dissertation of artifact detection and removal procedures and the effect of artifact on HRV. The performance of six artifact detection algorithms, including our newly developed algorithm, were assessed using the infant $R R$ interval data with real and artificial artifacts. Furthermore, the effect of artifacts on the HRV measurements was evaluated, particularly artifact which is difficult to detect.

\subsection{Background}

Artifacts in the RR interval will seriously distort HRV measures, especially frequency domain measurements. No matter how good the data acquisition system is, it is impossible to avoid artifacts. Some means of artifact detection and removal are generally necessary. There are two types of artifact detection methods that have been used in previous studies, visual inspection of both the ECG and RR interval signal and automatic artifact detection and correction algorithms. Visual examination is time consuming, even 
impractical for large datasets. In addition, the decision is dependent on the skill of the detector and may vary from person to person. Lastly, it is not an easy task to choose a proper criterion and quantitatively evaluate the performance of automatic detection algorithms. A combination of both automatic and manual approaches might be optimal. One study even indicated that manual inspection is necessary, because automatic algorithms are known to have potential errors [TASK96]. However, with the fast improvement of computer technology, it is now feasible to acquire, store and analyze large amounts of physiological data. In addition, the applications of heart rate and heart rate variability are rapidly increasing in clinical studies. An efficient automatic artifact removal routine is definitely needed. Furthermore, quantitatively investigating the impact of artifact on the HRV measures provides another way of evaluating the artifact removal procedure.

In RR interval data, the types of artifacts include missing beats, extra beats, misplaced triggers, environmental noise which makes the QRS complex difficult or even impossible detect properly, and physiologically abnormal beats (like premature ventricular contractions, sinus block etc.). The most critical aspect of this processing is to detect these artifacts accurately, because undetected artifacts will distort the measurement far more than even the crudest artifact correction method [BER90]. If the artifacts can be detected, several approaches can be used to correct them, such as measuring the actual RR interval from the ECG, interpolating from surrounding beats, or splitting a spuriously long beat into two (or more) equivalent RR intervals. As long as the incidence of the artifact is not too large, these approaches can be expected to introduce 
ignorable distortion in the $\mathrm{RR}$ interval time series and heart rate variability measures [CHE81, BER90, MAL95].

Undetected missing beats may cause far more serious biases. Even a single missing beat within a 2-min recording epoch can lead to errors in the HRV estimation [BER97, BER98]. For frequency domain analysis of HRV, more accurate detection of artifact is demanding. Especially when the value of HRV is small, because most of the energy in the power spectrum may be produced by few artifactual beats [BIG95]. Furthermore, the cost of a missed artifact, especially a missing beat, is often considerably greater than that of a false alarm. Figure 4.1 shows how one missing beat in 5-min segment of RR interval data affects the power spectral density of the RR interval and the frequency measurements of HRV using our infant data. 

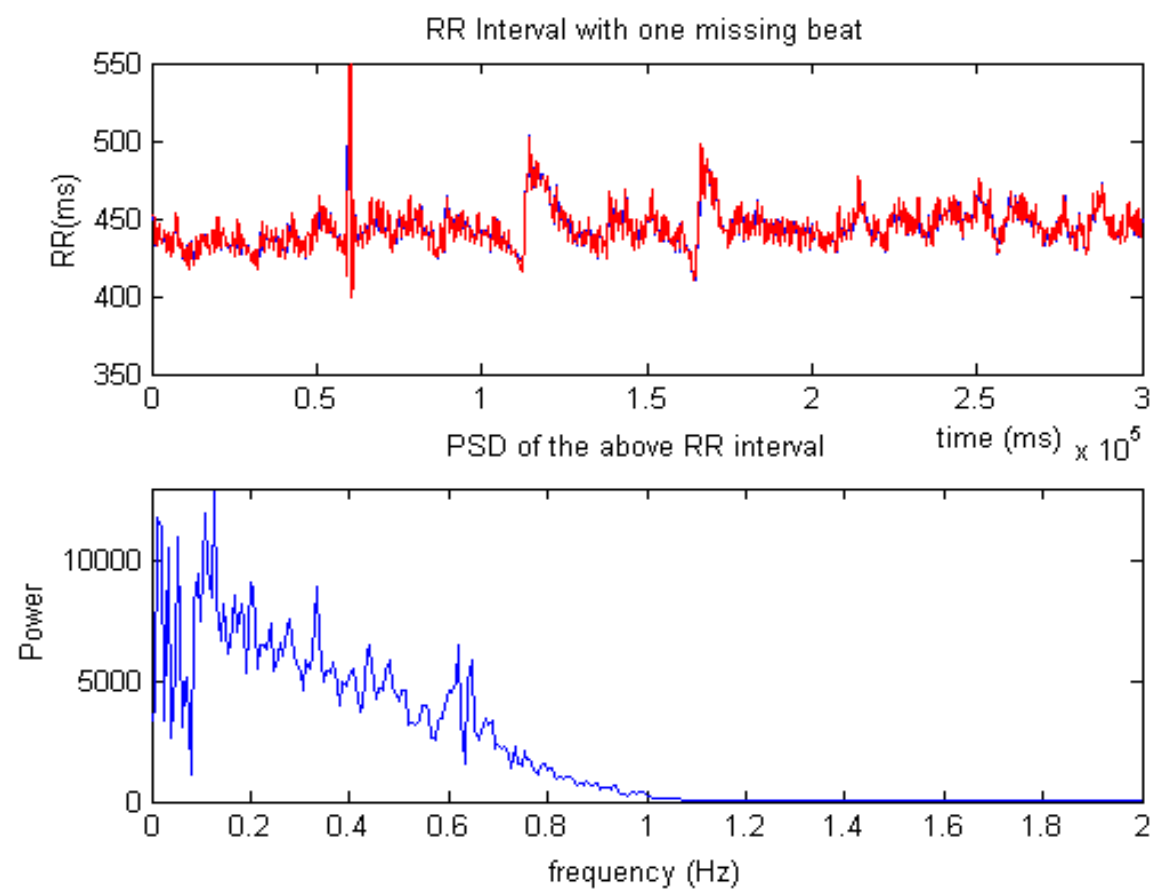

LF: 2.08E+05, NLF: 33.0373, HF: 4.22E+05, NHF: 66.9627, LF/HF ratio: 0.4934

(a) Signal with only one missing beat
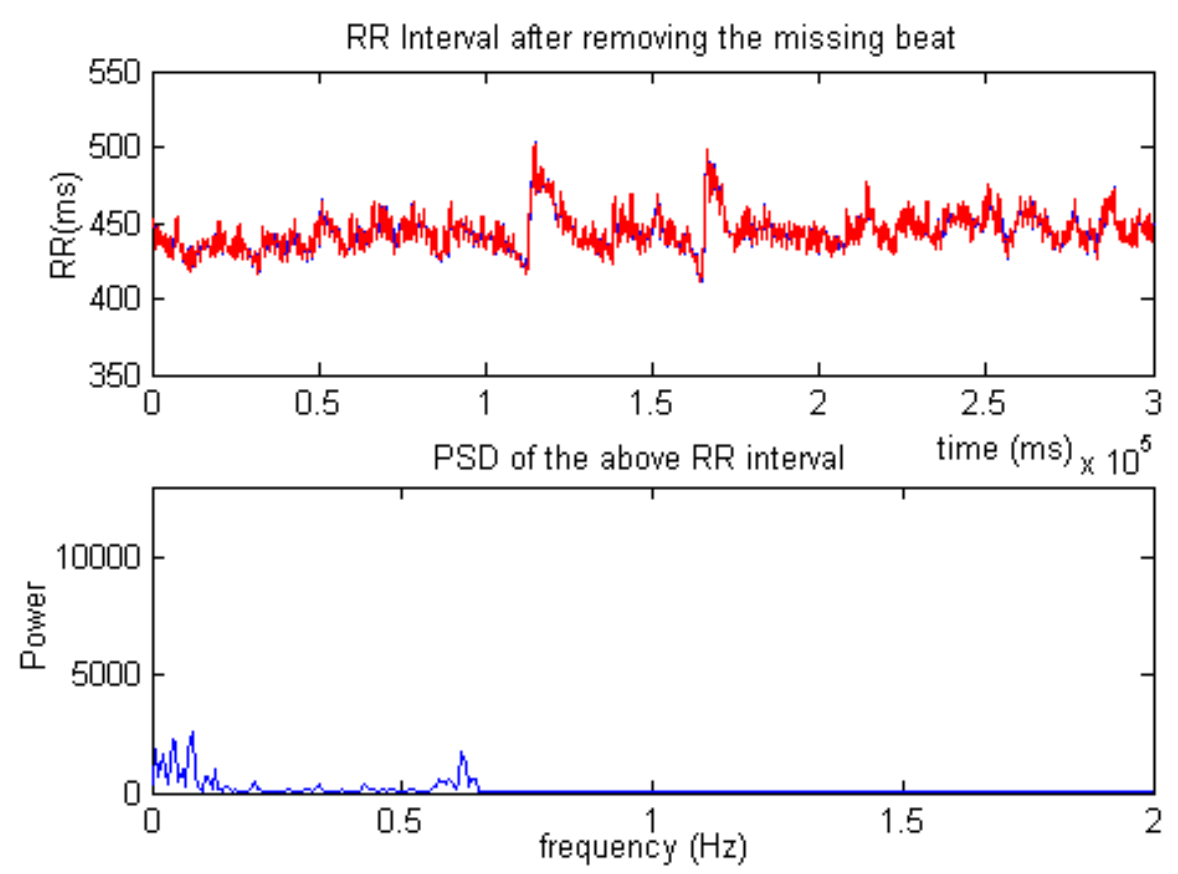

LF: 1.83E+04, NLF: 56.10, HF: 1.43E+04, NHF: 43.90, LF/HF ratio: 1.2777

(b) Missing beat removed by interpolation

LF is the low frequency power, NLF is the normalized LF, HF is the high frequency power, NHF is the normalized high $\mathrm{HF}$, and $\mathrm{LF} / \mathrm{HF}$ ratio is the ratio of $\mathrm{LF}$ to $\mathrm{HF}$

Figure 4.1 Effect of one missing beat on the power spectral density 


\subsubsection{Artifact identification}

There are many different criteria for artifact identification. The following are the most popular methods.

- Criteria based on the RR interval

In many methods, a fixed boundary of normal RR intervals (the maximum and minimum value of normal RR interval) is set according to the subject studied [HAD80, SAP91, LEI80]. Any RR intervals out of this range are considered artifacts. In another study, $\pm 25 \%$ of a five beat moving average was used to set the upper and lower limits in [LIA96]. In [LEI80], artifact RR intervals were eliminated from the data by automatically screening for intervals outside of the range and were verified by visually examining the original ECG. Fewer than $0.1 \%$ of RR intervals were rejected in their study.

In CHIME, the median of $25 \mathrm{RRs}$ surrounding each interval is used to set the range for normal $R R$ interval. If the difference between the current $R R$ interval and the median is greater than $30 \%$ of the median, this RR interval will be considered an artifact. If it is out of this range, it will be replaced by the median.

Cao et al. integrated three artifact detectors, limit-based, deviation-based and correlation-based detectors, to develop a parametric artifact detector called CVDetector for detecting artifacts in heart rate (HR) and mean blood-pressure (BP) data. The limitbased detector is to set an acceptable range for normal HR and BP. If wide limits are used for limit-base detector, it may have a high sensitivity, but lack of specificity. Reduced limits will resulted in better specificity, but decreased sensitivity. The deviation-based detector was added to improve the accuracy of the limit-based detector. It monitors variance of the data. Correlation-based detector was introduced in their study because 
cardiovascular parameters, such as $\mathrm{HR}$ and $\mathrm{BP}$, are correlated. A HR artifact will be claimed when its value passed a chosen threshold and a corresponding BP artifact was detected. HR and BP data from 10 preterm infants were used to test the CVDetector. They achieved $94.8 \% \pm 7.6 \%$ sensitivity and $90.6 \% \pm 6.9 \%$ specificity for HR [CAO99].

- Criteria based on $\Delta \mathrm{RR}$

Many criteria for artifact identification in RR interval data are defined based on the beat-to-beat difference, because beat-to-beat difference is less contaminated by linear trends, and yields the lowest overall variance [BER90, CHE81, KLE87, MAL89, SAP91].

In [BER90], two parameters were used to form the criterion index: Maximum Expected Difference (MED) for non-artifactual beats and Minimal Artifact Difference $(\mathrm{MAD})$, which are defined as: $\mathrm{MED}=3.32 * \mathrm{IQR}(\Delta \mathrm{RR}) / 2$ and $\mathrm{MAD}=(\operatorname{Median}(\mathrm{RR})-$ 2.9* $\mathrm{IQR}(\Delta \mathrm{RR}) / 2) / 3$, where $\mathrm{IQR}$ indicates for interquartile range. The criterion difference score was then defined as: (MAD+MED)/2. They demonstrated that this is a highly effective and conservative index, which identified every artifact in their test set, and yielded a low false alarm rate. They also designed and implemented a false alarm detection algorithm to improve specificity of the artifact detection.

Cheung compares the beat-to-beat differences with the previous RR interval, if the increase is greater then $32.46 \%$ or the decrease is more than $24.51 \%$, it will be marked as artifact [CHE81]. Similarly, Kleiger et al. used a $20 \%$ criterion for both increasing and decreasing differences when compared with the previous accepted RR interval [KLE87, KLE90]. 
Eight methods were studied in [SAP91]. Five of them were based on only RR intervals. They compared each RR with an epoch mean or moving mean with fixed or changing threshold. One method used a 51 order moving polynomial to fit the RR series, and defined artifact as the RRs which deviate by more than a threshold from the polynomial. Three methods are $\Delta \mathrm{RR}$ based, where the absolute $\Delta \mathrm{RR}$ is compared with the last accepted RR, or with both the last accepted RR and the updated mean. A fixed threshold, such as $20 \%$ or $30 \%$, was used to detect artifacts. Their results showed that short sudden changes can be detected successfully by most of the methods, but longer periods of noise contaminated with slow RR changes are difficult to detect.

Five algorithms were introduced in [MAL89], which compared difference of the target RR with previous, succeeding, last accepted RR interval, and /or the mean of all accepted RR intervals to see whether the difference is outside a threshold. Different thresholds (from 0.02 to 0.80 at a interval of 0.02 ) were tested for all five algorithms. They found that the algorithms using neighboring RR intervals fail when an abnormality or artifact is repeated over a long period of time. Using the last accepted RR interval can overcome this problem, but it is very sensitive to its own failures. Once it accepts an artifactual RR interval as good beat, it will detect a large series of real RR intervals as artifact by comparison with the artifact. They found that the algorithms, which used the last accepted and the mean of all the accepted RR intervals, works the best with the threshold at 0.08 and 0.1 .

These are the most commonly used methods to define an automatic routine for artifact detection in RR intervals. However, most of these methods still require manual inspection to verify and modify the results. In addition, these algorithms have difficulties 
with some aspects of our data, and did not achieve satisfactory detection accuracy in our infant data. Furthermore, due to the massiveness of our dataset, visual inspection is impossible. Thus, this provided the motivation to develop and study a new automatic algorithm based on previously developed techniques to improve the performance of artifact detection.

\subsubsection{Artifact correction}

Artifact in RR series cannot simply be deleted because this would disturb the continuity of the time series that is necessary for analysis of rhythmical variations. Therefore, after artifact is detected, the next step is to derive an accurate expected value for the artifactual RR intervals. Because RR intervals are often subject to phasic trends, respiratory sinus arrhythmia (RSA), or event-related heart rate responses, the averaged RR interval over a long epoch can not provide a good estimation of an individual beat within that epoch. The immediately surrounding beats may therefore offer the most viable expected value for a target beat. There is more than one way to correct the artifactual RR interval.

- The first method is to eliminate the artifact RR and keep all the other good RR intervals to preserve the timing relationships of the RR interval time series. This will not change the continuity of the time series. For each 5-min RR interval signal of infant, there are approximate $700 \mathrm{RR}$ intervals in it. Removal of several intervals will not affect our analyses much, especially in frequency domain, where resampling will be done to the RR intervals, and serves as interpolation for the removed RR. 
- The second is to replace the artifact using the median of the RRs surrounding the artifact.

- A third method is to use interpolation of the surrounding good beats [HAR76, BER97, HAR76, ROT90, STE88, BIG92, BIG89]. Two common methods are linear and cubic spline interpolation. We choose cubic spline interpolation to correct the artifacts in our data.

○ Linear Interpolation Method [MAL95]

$$
y(n)=y(n-1)+(y(n+1)-y(n-1)) * \frac{x(n)-x(n-1)}{x(n+1)-x(n-1)}
$$

Where $y(n)$ is the artifact $R R, y(n-1)$ is the previous accepted $R R, y(n+1)$ is the first accepted RR after the artifact, $x$ is the $x$-axis of these RRs, which is the time they occurred.

- MATLAB cubic spline interpolation method, also using the previous accepted RR interval and the first accepted RR interval after the artifact [PRE92, BOO78].

$$
\begin{gathered}
y(n)=A \cdot y(n-1)+B \cdot y(n+1)+C \cdot y^{\prime \prime}(n-1)+D \cdot y^{\prime \prime}(n+1) \\
\text { where } A=\frac{x(n+1)-x(n)}{x(n+1)-x(n-1)}, B=\frac{x(n)-x(n-1)}{x(n+1)-x(n-1)}, \\
C=\frac{1}{6}\left(A^{3}-A\right)(x(n+1)-x(n-1))^{2}, \text { and } D=\frac{1}{6}\left(B^{3}-B\right)(x(n+1)-x(n-1))^{2}
\end{gathered}
$$

Compared with linear interpolation, the cubic spline interpolation uses a formula that is smooth in the first derivative, and continuous in the second derivative. It is a more practical interpolation method. 


\subsubsection{Data rejection}

After detecting artifacts and replacing the bad beats by interpolation, an objective criterion is still needed for rejecting a data series from further analysis if there are too many interpolated values. Schechtman et al. rejected all 1-min epochs with more than 30 artifacts, or several artifacts that could not be corrected by combining intervals [SCH92]. Rottman et al. required the artifact rate in a 5-min segment to be less than 20\%; otherwise, the segment will be excluded in further HRV analysis [ROT90]. Less than $10 \%$ artifact in 512 samples' segment was accepted in [HUI94]. The criterion defined for our data is if the artifact to total beat ratio is greater than $10 \%$ in a 5 -min segment, this segment will be ignored.

\subsection{Material and Methods}

From visual inspection of the ECG and RR interval data in our dataset, most of our data is of high quality. However, there are times when the data contains missing beats, extra beats, abnormal beats, and noise contaminated periods. In addition, the amount of data which needs to be processed in our project is very large. Developing and implementing a suitable automatic algorithm to detect and remove artifacts for our data is an important part of our work. Several artifact identification algorithms, which have been used in the previous studies, have been evaluated for detection of artifacts in our data, but most have problems in our data. The most serious problem is that they sometimes detect as many good beats as bad beats, because some algorithms are very sensitive to their own failure. 
We developed an efficient, purely automatic algorithm to detect artifact in RR interval by combining previously developed criteria. We are interested in comparing our newly developed algorithm with previous artifact detection algorithms. All the algorithms are described in 4.3. Sensitivity, specificity and accuracy is used to quantify the performance of the algorithms. Both real artifacts and artificial artifacts in the infant RR interval data are used in the evaluation of the performance of these artifact detection algorithms.

\subsubsection{Dataset with real artifacts}

To form the dataset with real artifacts, 10 infants were randomly chosen from a large sample of 388 infants from the Collaborative Home Infant Monitoring Evaluation (CHIME) study. Of the 10 infants, there are two each from preterm, sudden infant death syndrome (SIDS) siblings and apparent life-threatening event (ALTE) groups and four from the normal infant group. Thirty minutes of simultaneous ECG and RR interval data of these 10 infants were used to test the performance of the algorithms. For each infant, 15 minutes were chosen randomly and 15 minutes were visually selected from noisy segments. There are many real artifacts in these infant RR interval data. The ECG was used to visually scan and classify good and bad beats for each segment. The artifacts are categorized into 6 types: (1) missing beats, (2) extra beats, (3) misplaced triggers, (4) noise where the QRS is discernable, (5) noise where the QRS is undetectable, and (6) abnormal beats (including block, premature beats, and other arrhythmias). Figure 4.2 shows the typical types of artifacts in our real data. Sensitivity of the algorithms to each kind of artifact, as well as the overall sensitivity, specificity, and accuracy were 
calculated. Comparison was done between the result of each automatic algorithm and the artifact classified manually from the ECG.

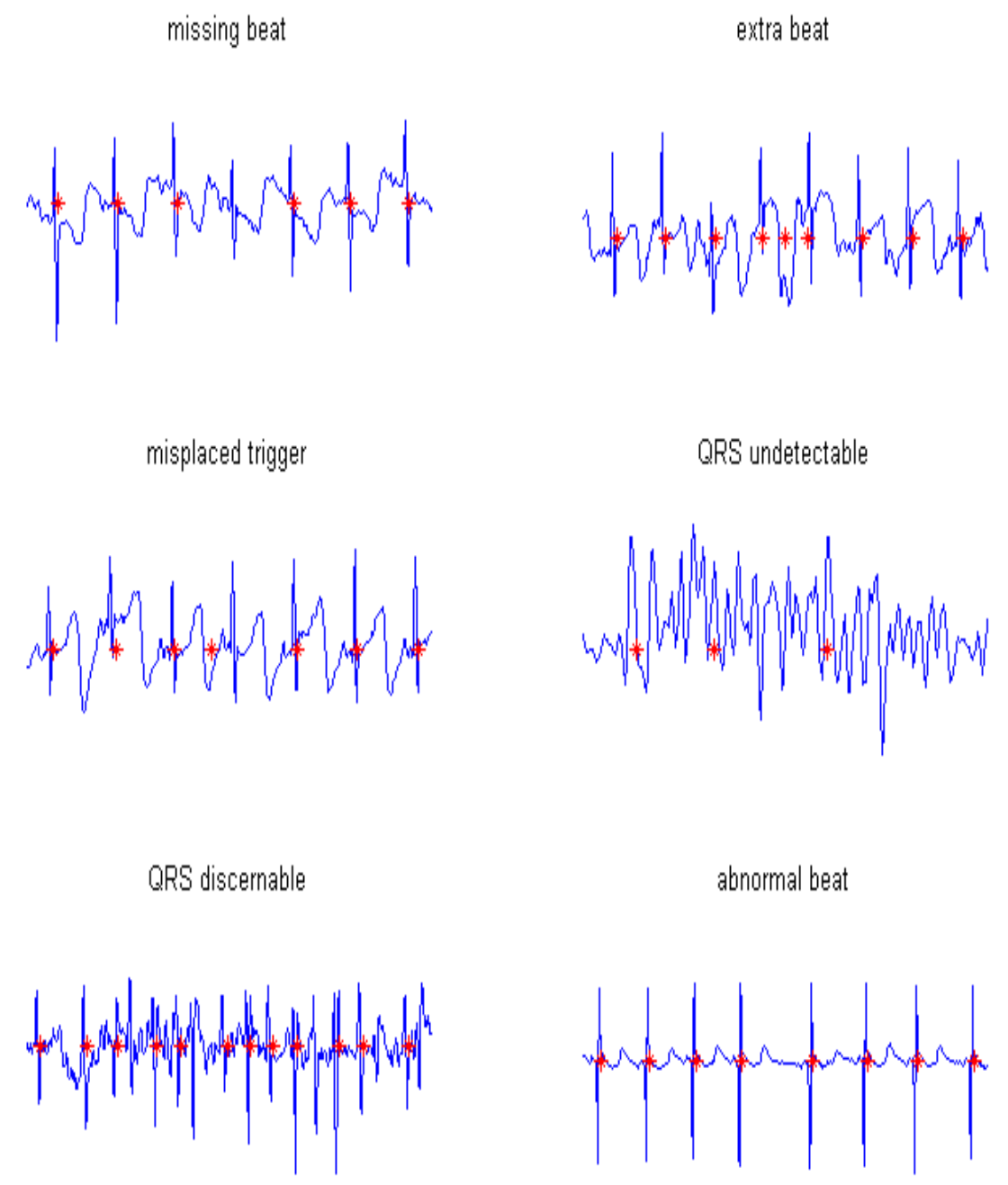

Figure 4.2 Six types of artifact in our data

\subsubsection{Dataset with artificial artifacts}

When using the dataset with real artifacts to evaluate the algorithms, the performance of the algorithms relies somewhat on the manual classifier's personal decision of whether a RR interval is a good beat or an artifact. The inevitable mistakes 
and other unknown or unexpected factors might affect the evaluation. Missing beats and extra beats are the major artifacts in RR interval data, and tend to affect the HRV measures the most. Therefore, in this study the performance of the artifact detection algorithms is tested on a dataset of artificially generated missing beats and extra beats added to clean data.

To construct a dataset with artificial artifacts, the same 15-minute randomly chosen RR intervals from ten infants are used. Most of these data contain few real artifacts. The segments of data are cleaned by interpolation first. Then, a controlled number and type of artificial artifacts are randomly added to the clean RR intervals to test the artifact detection algorithms. Although the removal of the real artifacts of each segment also depends on the personal decision of whether it is a "real" artifact, this will not affect the evaluation of the algorithms, because this is just a way of getting clean real RR interval data. The added artificial artifacts are what is used to assess the algorithms. The performance of the algorithms depends on whether they detect the artificial artifacts correctly or incorrectly.

Missing beats and extra beats are the types of artifacts with which we are most concerned. Scattered artifacts and artifacts in a row are treated differently, since it might have different influences on artifact detection algorithms and the HRV measurements. Thus, there are four kinds of artificial noise that is generated and added to the real data: scattered missing beats, scattered extra beats, missing beats in a row, and extra beats in a row. The procedures to generate them are also different for different types of artifact. To create scattered artifacts, we (1) generate a random number to give the position of the artifacts; (2) find the RR interval at that position, add the next RR to it and delete the next 
RR to form a missing beat, or break the RR equally into two RRs to get two extra beats; and (3) repeat this procedure to generate as many scattered artifacts as needed. To generate $\mathrm{N}$ artifacts in a row, we (1) generate a random number to give the position of the artifacts; (2) start from the RR at that position, add the next two RRs to form a missing beat, or break the next RRs equally into two RRs to generate two extra beats; and (3) repeat for the next $\mathrm{RR}$ interval to form $\mathrm{N}$ or $2 \mathrm{~N}$ artifacts in a row. From the above procedure, the generation of an extra beat actually breaks one good RR into two extra beats. The number of extra beats is always double the number of artifacts you choose to generate. This must be taken into account when comparing the performance of the algorithms with the number of artifacts in the RR interval.

\subsection{Algorithms}

The following six algorithms were evaluated in this study. The newly developed algorithm is algorithm $\mathrm{f}$.

Algorithm a Each RR interval is compared with the last accepted RR interval. If the difference is outside of $\pm 20 \%$ of the last accepted RR, it is detected as artifact. This is the most commonly used method to detect artifact for RR interval data [KLE87, KLE90]. It is acceptable for most of the data. However, it might exclude a sinus interval in which there are sudden large changes in sinus rate, and as discussed above, this method is very sensitive to its own failures. Once it accepts an artifactual RR interval, it will omit a large series of real RR intervals because artifact is used for the comparison [MAL98]. Our results confirm this (see Figure 4.3). The red circles indicate the bad beats detected by the algorithms. 

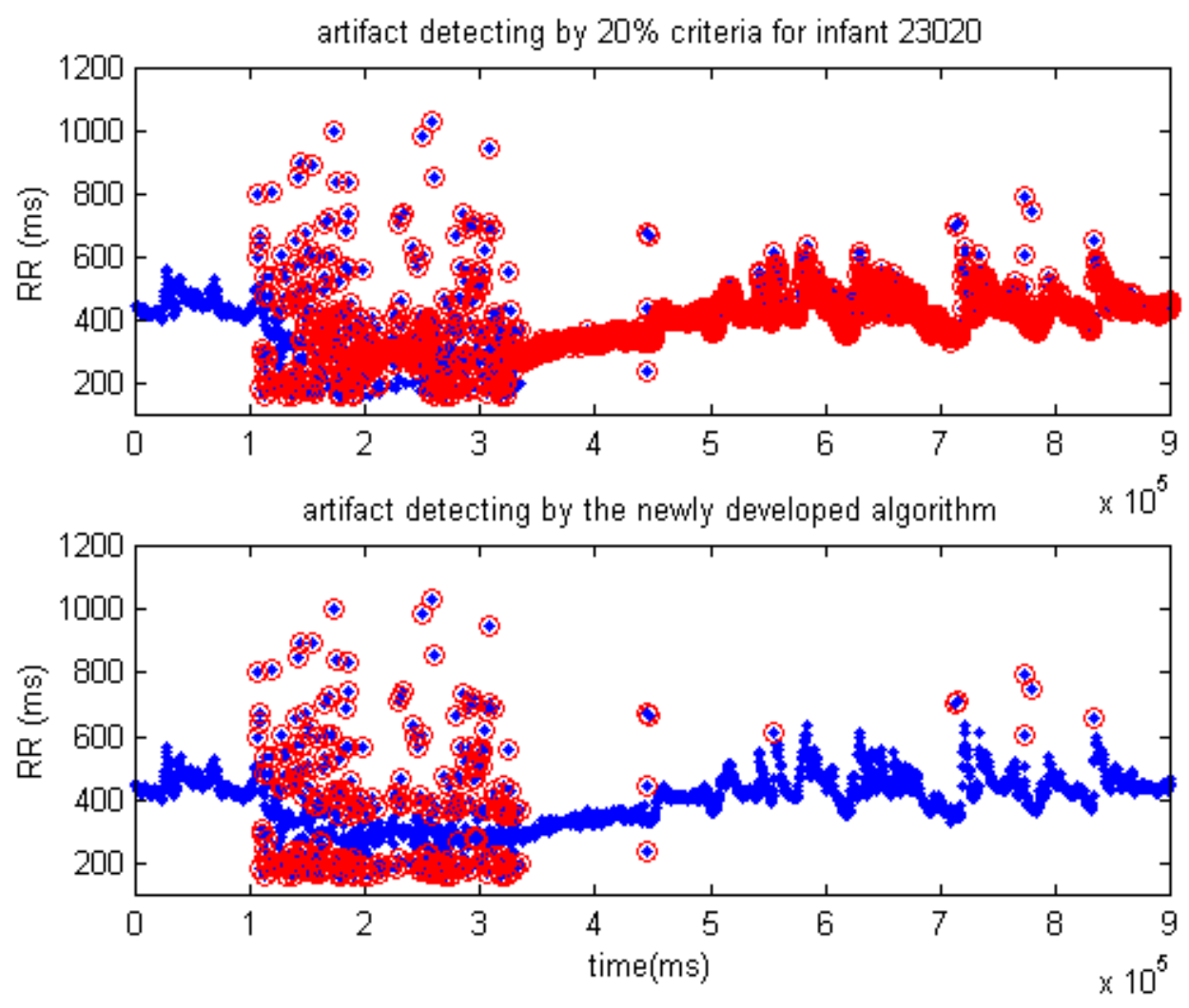

Figure 4.3 Sensitiveness of the algorithm to its own failure

The top graph is the results of artifact detection by Algorithm a for 15 minutes. The blue dots are RR intervals and the red circles indicate beats detected as artifact. The bottom graph is the newly developed Algorithm $\mathrm{f}$ which also uses a medium filter.

Algorithm b This algorithm is modified based on Algorithm a. It also uses the $20 \%$ criteria. Each RR interval is compared with the last two accepted RR intervals. If both differences are outside of $\pm 20 \%$, the $\mathrm{RR}$ interval is detected as artifact. The algorithm is based on the characteristic that the RR interval cannot change abruptly, but should follow the trend of the surrounding beats. By comparing with two accepted beats, it hopes to reduce the possibility of accepting an artifactual RR interval.

Algorithm c This algorithm uses a combination of two existing criteria. One criterion index is defined based on $\Delta \mathrm{RR}$ by calculating Maximum Expected Difference 
(MED) for non-artifactual beats and Minimal Artifact Difference (MAD). MED is defined as $3.32 \times \mathrm{IQR}(\Delta \mathrm{RR}) / 2$, while $\mathrm{MAD}$ is (median $(\mathrm{RR})-2.9 \times \mathrm{IQR}(\Delta \mathrm{RR}) / 2) / 3$. The criterion index is then defined as (MAD+MED)/2 [BER90]. The other criterion is to set the upper and lower limit for each $\mathrm{RR}$ interval using 5 beat moving mean $\pm 25 \%$ [LIA96]. Only if the RR is detected as a bad beat by both criteria, it will be detected as an artifact.

Algorithm d The median of 25 RRs surrounding each RR interval, the 12 before and 12 after, is used to set the range. If the difference between the current RR interval and the median is greater than $\pm 30 \%$ of the median, this RR is considered as an artifact. This is the method chosen by CHIME.

Algorithm e This is another algorithm modified based on Algorithm a. It attempts to solve the problem of detecting a large series of real RR intervals as artifacts. Every RR interval is compared with the mean of all the accepted RR intervals as well as the last accepted RR. If both differences are greater than $\pm 20 \%$, it will be treated as artifact [MAL89].

Algorithm f Since each RR should typically follow the trend of the surrounding RR intervals, this newly developed algorithm is based on Algorithm d and Algorithm e. Instead of using the mean of all accepted RR intervals to do the comparison, it compares each RR with the median of 25 surrounding $R R$ intervals and the last accepted RR interval. Only if both differences are outside $20 \%$ is it detected as artifact.

It should be noted that when implementing the algorithms which calculate a threshold based on single beats ( $a, b, e, f)$, the algorithm uses only "accepted" beats, that is, only beats which have been checked and are not classified as artifact. 


\subsection{Results and Discussion}

Sensitivity, specificity, and accuracy are used as a measure of the performance to compare the above algorithms. Sensitivity is the percentage of artifactual beats detected correctly. Specificity is the percentage of good beats detected correctly. The accuracy is the percentage of correct classification over the total number of beats.

Table 4.1 Truth table for the definition of sensitivity, specificity, and accuracy

\begin{tabular}{|c|c|c|}
\hline Algorithm Truth & Good Beat & Bad Beat \\
\hline Good Beat & TN & FN \\
\hline Bad Beat & FP & TP \\
\hline
\end{tabular}

$$
\begin{gathered}
\text { Sensitivity }=\frac{T P}{F N+T P} \quad \text { Specificity }=\frac{T N}{T N+F P} \\
\text { Accuracy }=\frac{T N+T P}{F N+T P+T N+F P}
\end{gathered}
$$

where TN stands for true negative, FN false negative, TP true positive, and FP false positive.

\subsubsection{Detecting real artifacts}

Tables 4.2, 4.3, and Figure 4.4 show the results of detecting artifacts on the entire real artifact dataset. We calculated the sensitivity of the algorithms to each type of artifact, as well as the overall sensitivity. The test dataset were divided into two groups, randomly chosen 15-min data, and visually selected 15-min noisy data. Table 4.2 provides the raw information regarding the number of good and bad beats and gives 
results of each of the algorithms. Table 4.3 gives sensitivity and specificity values for each of the algorithms and also includes results for each type of artifact. Figure 4.4 gives a graphical representation of sensitivity and specificity for each algorithm. From the result, we found that all algorithms work quite well on the randomly chosen data, which contain fewer artifacts. Some of the algorithms also work well on the noisy data.

Since the number of bad beats is not very large, missing just several bad beats results in decreased sensitivity. A major culprit for decreased sensitivity is type 4 and type 5 artifacts, noise where the QRS is discernable or undetectable, respectively. Type 4 and 5 artifacts are very difficult to detect, because the trigger (or beat detector value) is still placed somewhere even if the QRS is undetectable, and the RR interval calculated from these triggers is often very similar to real RR interval in value. However, these two types of artifact will have a small effect on HRV measurements since the RR interval values are near the real RR interval. This is explored in section 4.5.

The effect of artifact on HRV measurements is different depending on the type of artifact. Missing beats have the greatest effect over any other type of artifact. Furthermore missing beats and extra beats are the most commonly encountered artifact in heart period data. In evaluating the algorithms, the sensitivity to these two types of artifact is critical. A good algorithm should not miss many missing beats and extra beats. All algorithms except algorithm c, achieve very high sensitivity to missing beats and extra beats. Considering missing and extra beats only, $100 \%$ sensitivity for the random dataset is reached, and sensitivity ranges from 0.9103 to 1.0 and from 0.9286 to 1.0 for the noisy and the entire testing dataset, respectively (excluding algorithm c). Among them, the newly developed algorithm $\mathrm{f}$ is the best. 
Detecting many good beats as bad beats is also unacceptable. All the algorithms achieved very high specificity, i.e. the algorithms do not miss many good beats. In choosing an algorithm, there is a tradeoff between sensitivity and specificity. Figure 4.5 gives a graphical representation of sensitivity and specificity for each algorithm, in order to compare the algorithms. The graph includes the real artifact dataset both for missing beats and overall. In terms of specificity, algorithms $\mathrm{c}$ and $\mathrm{d}$ are better than $\mathrm{f}$, with the values 0.9939 and 0.9947 , respectively, versus 0.9917 for the entire dataset. However, the sensitivity of $\mathrm{c}$ and $\mathrm{d}$ is much lower than algorithm $\mathrm{f}, 0.5850$ and 06230 versus 0.7346 . Algorithm $\mathrm{f}$ provides the best balance of sensitivity and specificity.

Accuracy is another parameter which can be used in the evaluation of the performance of each algorithm. The accuracy of algorithm c, d, and $\mathrm{f}$ is much better than other three algorithms. Algorithm $d$ is the best, with accuracy of 0.9861. Algorithm $\mathrm{f}$ ranks second with an accuracy of 0.9857 .

Considering above factors, we conclude that the newly developed algorithm (f) is better than other algorithms for detecting real artifacts in this infant dataset. It works almost perfectly for detecting missing beats and extra beats $(100 \%$ and $97.44 \%$ sensitivity, respectively) even on the noisy data. For the entire testing dataset, it achieved 73.46\% overall sensitivity, $99.17 \%$ specificity and $98.57 \%$ accuracy, which provided the best overall performance. 


\begin{tabular}{|c|c|c|c|c|c|c|c|c|}
\hline $\begin{array}{c}\text { Random } \\
\text { Data }\end{array}$ & $\begin{array}{l}\text { Number of } \\
\text { beats }\end{array}$ & Algorithms & $\mathbf{a}$ & b & c & d & $\mathbf{e}$ & $\mathbf{f}$ \\
\hline Good Beats & 19428 & $\begin{array}{l}\text { Number of beats that } \\
\text { correctly detected }\end{array}$ & 19252 & 19289 & 19374 & 19372 & 19276 & 19339 \\
\hline Good Beats & & $\begin{array}{l}\text { Number of beats that } \\
\text { incorrectly detected }\end{array}$ & 176 & 139 & 54 & 56 & 152 & 89 \\
\hline Bad Beats & 79 & $\begin{array}{l}\text { Number of beats that } \\
\text { correctly detected }\end{array}$ & 75 & 74 & 68 & 65 & 71 & 74 \\
\hline Bad Beats & & $\begin{array}{l}\text { Number of beats that } \\
\text { incorrectly detected }\end{array}$ & 4 & 5 & 11 & 14 & 8 & 5 \\
\hline $\begin{array}{l}\text { Noisy } \\
\text { Data }\end{array}$ & $\begin{array}{l}\text { Number of } \\
\text { beats }\end{array}$ & Algorithms & $\mathbf{a}$ & b & c & d & $\mathbf{e}$ & $\mathbf{f}$ \\
\hline Good Beats & 19631 & $\begin{array}{l}\text { Number of beats that } \\
\text { correctly detected }\end{array}$ & 17324 & 17841 & 19448 & 19479 & 19154 & 19394 \\
\hline Good Beats & & $\begin{array}{l}\text { Number of beats that } \\
\text { incorrectly detected }\end{array}$ & 2307 & 1790 & 183 & 152 & 477 & 237 \\
\hline Bad Beats & 844 & $\begin{array}{l}\text { Number of beats that } \\
\text { correctly detected }\end{array}$ & 630 & 599 & 472 & 510 & 579 & 604 \\
\hline Bad Beats & & $\begin{array}{l}\text { Number of beats that } \\
\text { incorrectly detected }\end{array}$ & 214 & 245 & 372 & 334 & 265 & 240 \\
\hline
\end{tabular}

\begin{tabular}{|c|c|c|c|c|c|c|c|c|}
\hline $\begin{array}{c}\text { All } \\
\text { Data }\end{array}$ & $\begin{array}{c}\text { Number of } \\
\text { beats }\end{array}$ & Algorithms & a & b & c & d & e & f \\
\hline Good Beats & 39059 & $\begin{array}{c}\text { Number of beats that } \\
\text { correctly detected }\end{array}$ & 36576 & 37130 & 38822 & 38851 & 38430 & 38733 \\
\hline Good Beats & 923 & $\begin{array}{c}\text { Number of beats that } \\
\text { incorrectly detected }\end{array}$ & 2483 & 1929 & 237 & 208 & 629 & 326 \\
\hline Bad Beats & $\begin{array}{c}\text { Number of beats that } \\
\text { correctly detected }\end{array}$ & 705 & 673 & 540 & 575 & 650 & 678 \\
\hline Bad Beats & $\begin{array}{c}\text { Number of beats that } \\
\text { incorrectly detected }\end{array}$ & 218 & 250 & 383 & 348 & 273 & 245
\end{tabular}

Table 4.2 Performance of the algorithms for random data, noisy data and all testing dataset 
Sensitivity, specificity and accuracy of the algorithms on random data

\begin{tabular}{|c|c|c|c|c|c|c|}
\hline & Algorithm a & Algorithm b & Algorithm c & Algorithm d & Algorithm e & Algorithm $f$ \\
\hline SENS1 & 1.0000 & 1.0000 & 1.0000 & 1.0000 & 1.0000 & 1.0000 \\
\hline SENS2 & 1.0000 & 1.0000 & 0.6500 & 1.0000 & 1.0000 & 1.0000 \\
\hline SENS3 & 0.9200 & 0.9000 & 0.9200 & 0.7200 & 0.8400 & 0.9000 \\
\hline SENS4 & 1.0000 & 1.0000 & 1.0000 & 1.0000 & 1.0000 & 1.0000 \\
\hline SENS5 & 1.0000 & 1.0000 & 1.0000 & 1.0000 & 1.0000 & 1.0000 \\
\hline SENS6 & 1.0000 & 1.0000 & 1.0000 & 1.0000 & 1.0000 & 1.0000 \\
\hline SENS & 0.9494 & 0.9367 & 0.8608 & 0.8228 & 0.8987 & 0.9367 \\
\hline SPEC & 0.9909 & 0.9928 & 0.9972 & 0.9971 & 0.9922 & 0.9954 \\
\hline Accuracy & 0.9908 & 0.9926 & 0.9967 & 0.9964 & 0.9918 & 0.9952 \\
\hline
\end{tabular}

Sensitivity, specificity and accuracy of the algorithms on noisy data

\begin{tabular}{|c|c|c|c|c|c|c|}
\hline & Algorithm a & Algorithm b & Algorithm c & Algorithm d & Algorithm e & Algorithm f \\
\hline SENS1 & 0.9722 & 0.9861 & 0.9444 & 0.9861 & 0.9861 & 1.0000 \\
\hline SENS2 & 0.9103 & 0.9744 & 0.6026 & 0.9359 & 0.9744 & 0.9744 \\
\hline SENS3 & 0.7327 & 0.7512 & 0.6774 & 0.6313 & 0.6083 & 0.7788 \\
\hline SENS4 & 0.6429 & 0.6429 & 0.3571 & 0.4286 & 0.7143 & 0.5714 \\
\hline SENS5 & 0.6933 & 0.6048 & 0.4428 & 0.4816 & 0.6263 & 0.6026 \\
\hline SENS6 & 1.0000 & 1.0000 & 1.0000 & 1.0000 & 1.0000 & 1.0000 \\
\hline SENS & 0.7464 & 0.7097 & 0.5592 & 0.6043 & 0.6860 & 0.7156 \\
\hline SPEC & 0.8825 & 0.9088 & 0.9907 & 0.9923 & 0.9757 & 0.9879 \\
\hline Accuracy & 0.8769 & 0.9006 & 0.9729 & 0.9763 & 0.9638 & 0.9767 \\
\hline
\end{tabular}

Sensitivity, specificity and accuracy of the algorithms on all test dataset

\begin{tabular}{|c|c|c|c|c|c|c|}
\hline & Algorithm a & Algorithm b & Algorithm c & Algorithm d & Algorithm e & Algorithm $\mathrm{f}$ \\
\hline SENS1 & 0.9753 & 0.9877 & 0.9506 & 0.9877 & 0.9877 & 1.0000 \\
\hline SENS2 & 0.9286 & 0.9796 & 0.6122 & 0.9490 & 0.9796 & 0.9796 \\
\hline SENS3 & 0.7678 & 0.7790 & 0.7228 & 0.6479 & 0.6517 & 0.8015 \\
\hline SENS4 & 0.6429 & 0.6429 & 0.3571 & 0.4286 & 0.7143 & 0.5714 \\
\hline SENS5 & 0.6933 & 0.6048 & 0.4428 & 0.4816 & 0.6263 & 0.6026 \\
\hline SENS6 & 1.0000 & 1.0000 & 1.0000 & 1.0000 & 1.0000 & 1.0000 \\
\hline SENS & 0.7638 & 0.7291 & 0.5850 & 0.6230 & 0.7042 & 0.7346 \\
\hline SPEC & 0.9364 & 0.9506 & 0.9939 & 0.9947 & 0.9839 & 0.9917 \\
\hline Accuracy & 0.9324 & 0.9455 & 0.9845 & 0.9861 & 0.9774 & 0.9857 \\
\hline
\end{tabular}

SENS1-6: sensitivity to artifact types 1-6; SENS: sensitivity to all kinds of artifacts; SPEC: specificity

Table 4.3 Sensitivity, specificity and accuracy of the six algorithms for each type of artifact and combined artifact 


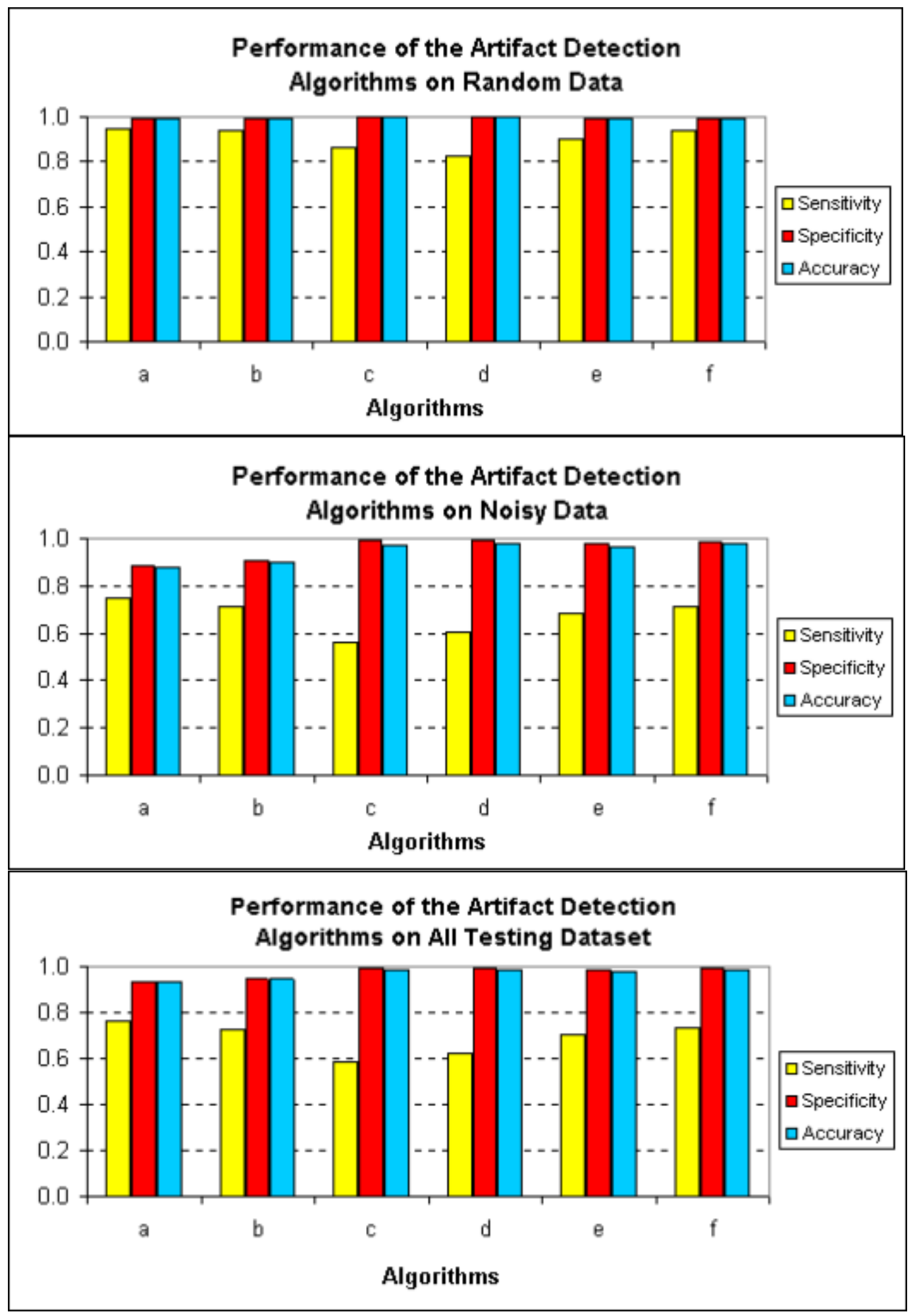

Figure 4.4 Sensitivity, specificity and accuracy of the six algorithms 


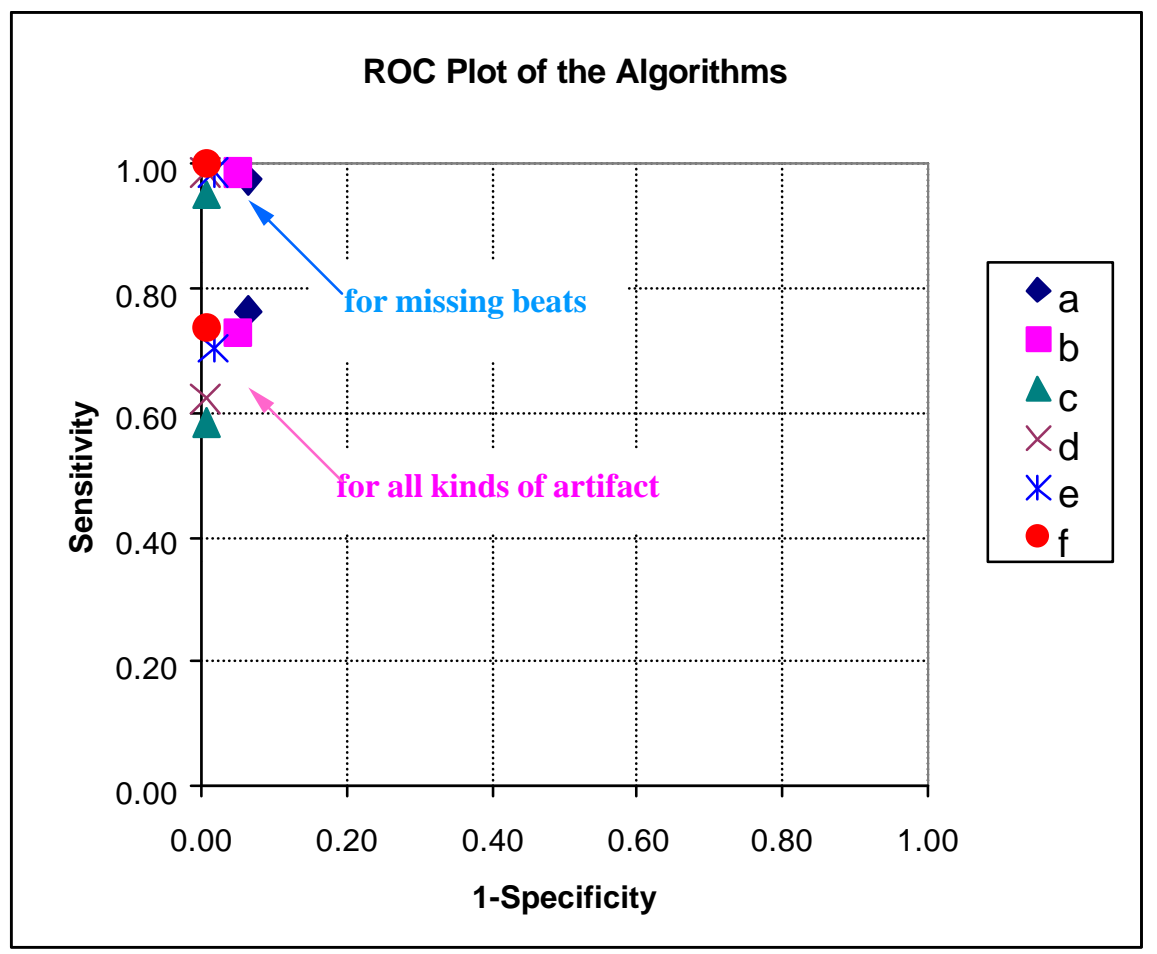

Figure 4.5 Relative operating characteristic curve which compares various algorithms

From the above results, the newly developed algorithm works better than any other algorithms if sensitivity, specificity, and accuracy are considered together to evaluate the algorithms. However, from these values, it is difficult to tell whether there is significant difference between these algorithms for artifact detection. A chi-square test is used to compare whether there is a significant difference between the results of these algorithms.

The results from the chi-square test shows that there are significant differences between these six algorithms in detecting good and bad beats for random, noisy, and the entire testing datasets $(\mathrm{p}<0.001)$. When comparing any two algorithms, all of the 
combinations for noisy data and overall results are significantly different $(\mathrm{p}<0.005)$, except comparisons between algorithm $\mathrm{c}$ and $\mathrm{d}$. When dealing with random data, which contains fewer artifacts, the performances of some algorithms are similar, where there is no significant difference in the results between algorithms $b$ and e, or between $c$ and $d$. No matter which dataset is considered, the difference between our newly developed algorithm $\mathrm{f}$ and any other algorithms is significant $(\mathrm{p}<0.005)$.

\subsubsection{Detecting artificial artifacts}

To evaluate the algorithm's ability to detect artificial artifacts, up to 20 artificial missing beats and extra beats are added to the real RR interval data on 10 infants. Sensitivity and specificity are calculated for each algorithm. Figure 4.6 and Appendix B show the results of detecting artificial artifacts. Consistent with the results of real artifacts, all algorithms, except algorithm c, achieve $100 \%$ sensitivity in detecting artificial scattered missing beats and extra beats. Algorithm c has the sensitivity of $100 \%$ for detecting scattered missing beats, but only $50 \%$ sensitivity is reached for scattered extra beats detection.

Figure 4.6 (a) and (b) show the changes of overall sensitivity with the number of missing beats in a row, and scattered missing beats. For missing beats and extra beats in a row, all algorithms achieve $100 \%$ sensitivity except algorithm c, algorithm $\mathrm{d}$ and the newly developed algorithm f. Algorithms d and f achieve 100\% sensitivity until there are more than 13 artifacts in a row. At this point, since the algorithms use the median of the surrounding $25 \mathrm{RR}$ intervals to set the threshold, a "bad beat" becomes the median causing mistakes. A solution would be to off-center the median window, so that at least 
13 intervals are ones which have been checked (whether they are artifact or not) and corrected. This solution will need to be tested. Note that this situation was not encountered in the real artifact dataset. Algorithm c, which uses a 5-beat moving mean and $\Delta$ RR-based criterion index to set the threshold, has a problem when detecting artifacts in a row and results in decreasing sensitivity with the number of artifacts in a row. Both the 5-beat moving mean and the other criterion based on $\Delta \mathrm{RR}$ may use artifact as part of the calculation. All other algorithms used only good or "accepted" beats for threshold calculation, except algorithms based on the median, which is robust to artifact. This is also the reason for $50 \%$ sensitivity in detecting scattered extra beats, because an extra trigger always resulted in two artifacts in a row.

In terms of specificity, there is not much difference among the algorithms and he types of artifact. A representative example of the results for specificity is shown in Figure 4.6 (c). The overall specificity of the six algorithms ranges from 0.9902 to 0.9974 . The newly developed algorithm f ranks third among the algorithms. This is consistent with the real artifact dataset, where specificity for algorithm $\mathrm{f}$ is third after algorithm $\mathrm{c}$ and $\mathrm{d}$. However, algorithm $\mathrm{f}$ has better sensitivity than algorithms $\mathrm{c}$ and $\mathrm{d}$ on the real artifact dataset and has better sensitivity than algorithm $\mathrm{c}$ on atificial artifact dataset. In addition, the specificity changes little with the number of artifacts for all the algorithms and is more dependent on the original RR interval data of the infants.

A chi-square test was applied to the results of detecting six artifacts added to the RR intervals. There is a significant difference between all pairs of the six algorithms $(\mathrm{p}<0.05)$, except between algorithms a and $\mathrm{b}$, between $\mathrm{c}$ and $\mathrm{d}$, and between e and $\mathrm{f}$. 


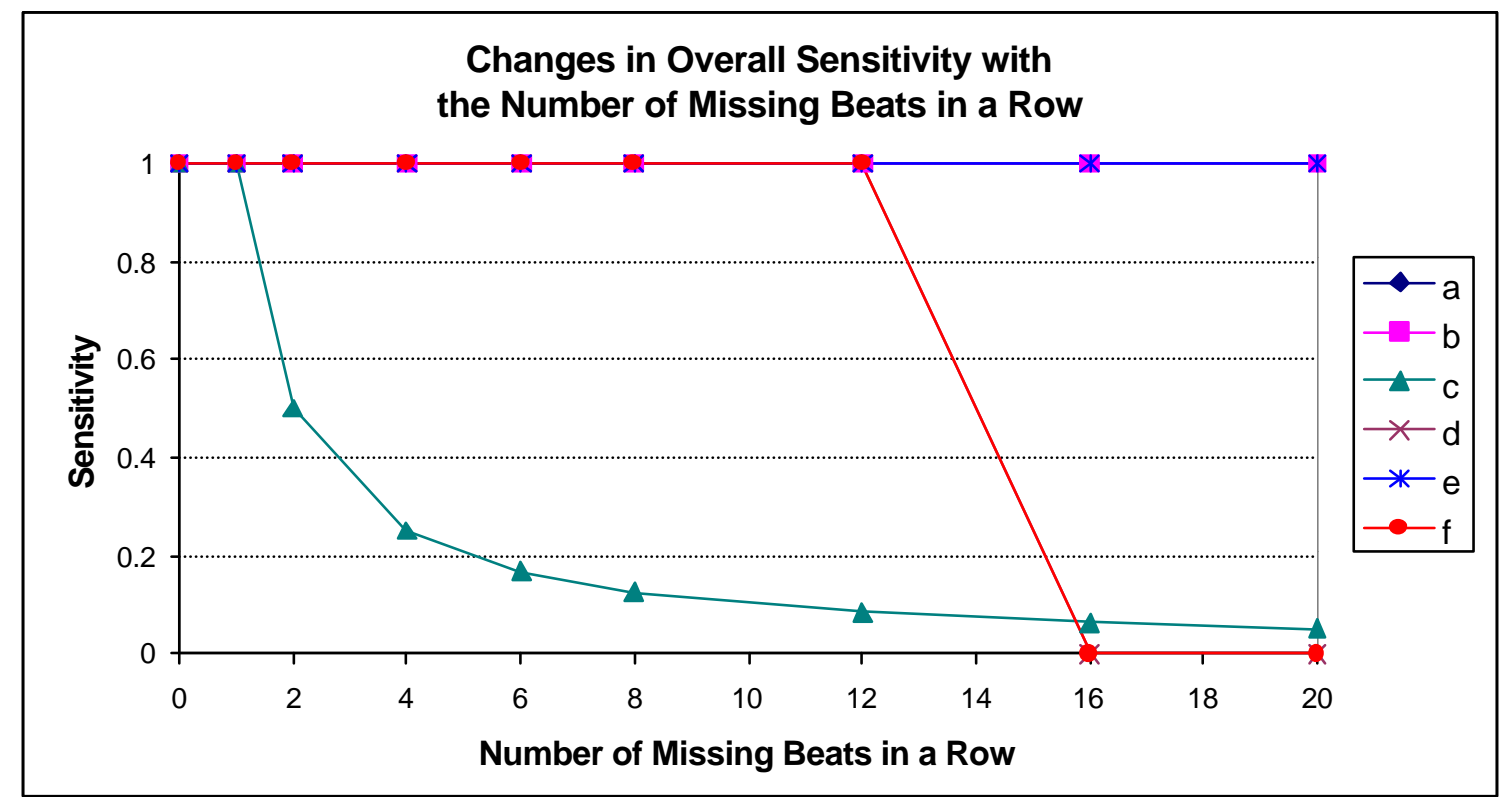

Note: Algorithm a, b, and e achieved 100\% sensitivity (for all amounts of artifact). Sensitivity of algorithm $\mathrm{d}$ and $\mathrm{f}$ is $100 \%$ until there are more than 13 artifacts in a row where the sensitivity then drops to 0 .

(a)

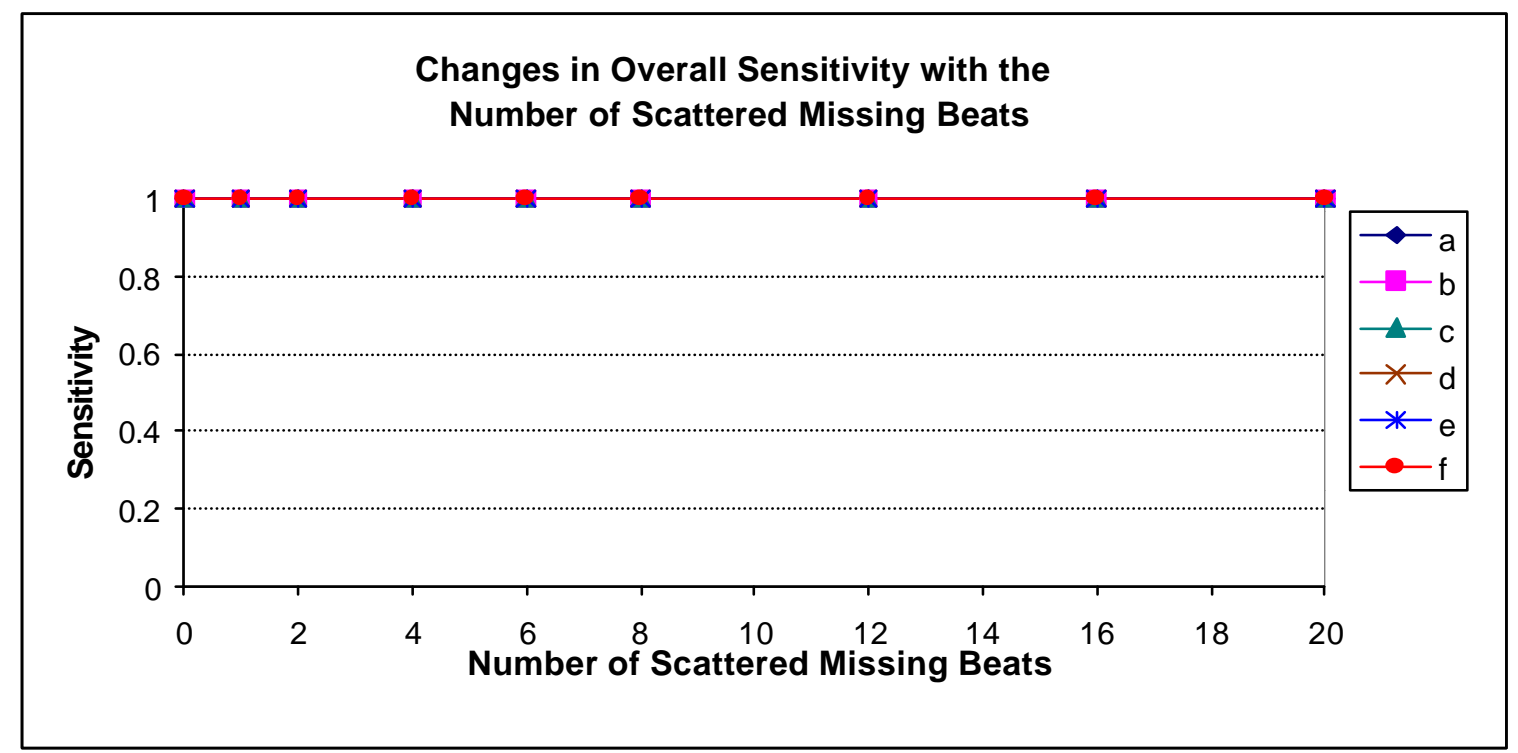

Note: All algorithms achieved 100\% sensitivity.

(b)

Figure 4.6 Changes of sensitivity \& specificity of various algorithms with different amounts of artificial artifacts 


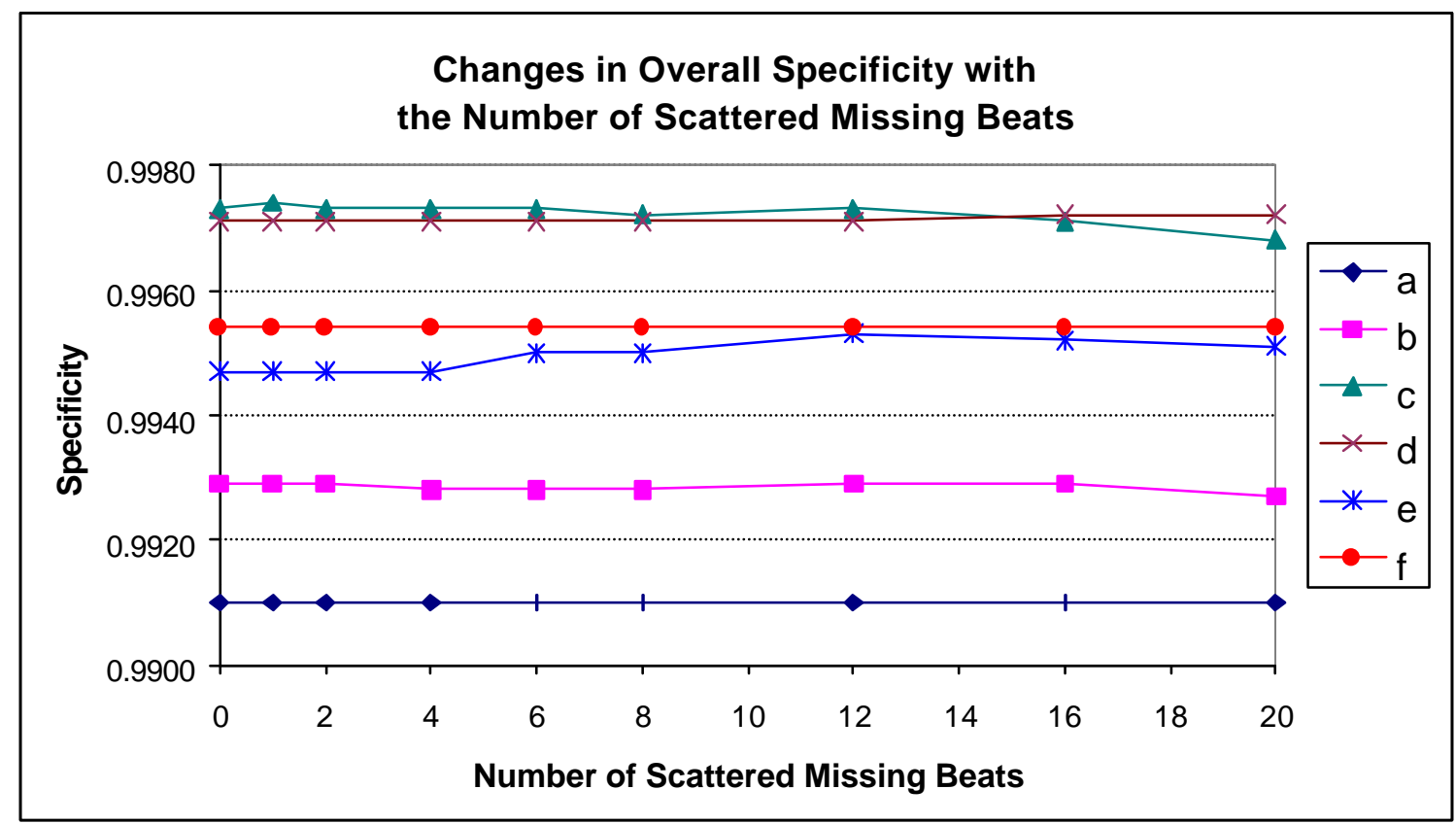

Note: The Y axis range is only from 0.99 to 0.998

(c)

Figure 4.6 Changes of sensitivity \& specificity of various algorithms with different amounts of artificial artifacts (Cont.)

\subsubsection{Conclusion}

In summary, the newly developed algorithm performs better than other algorithms for detecting real artifacts in infant RR interval data, providing the best balance between sensitivity and specificity $(73.46 \%$ and $99.17 \%$, respectively), greatly improving specificity with only a slight loss in sensitivity. It works extremely well for detecting missing and extra beats (100\% and $97.44 \%$ sensitivity, respectively) even on very noisy data. In terms of detecting artificial missing beats and extra beats, high sensitivity is achieved in most situations, and there is not much difference in specificity among the algorithms. One potential drawback to algorithm $\mathrm{f}$ is that it will make mistakes when 
encountering more than 12 artifacts in a row. A solution is suggested and should be tested.

\subsection{Evaluating HRV Parameters in the Presence of Noise}

While evaluating the HRV parameters, one of the important issues is how HRV parameters are affected by noise, and which is a more reliable measure for use with noisy data. For instance, compared with mean and standard deviation, median and interquartile range are less sensitive to the presence of extreme values in the data, and frequency domain measures of $\mathrm{HRV}$ tend to be more sensitive to noisy data than time domain measures. We are interested in comparing the changes of HRV parameters with a controlled number of artifacts in the RR interval.

A similar dataset to the last section of clean real RR interval data with artificial artifacts is used to investigate the effect of artifact on the HRV parameters. Since most heart rate variability (HRV) calculations and analyses are based on consecutive 5 minute segments of RR interval in this project, the first 5 minutes of 15 minutes of randomly chosen RR intervals from 10 infants were used to study the effects of artifacts on the HRV parameters. All HRV parameters are computed for the 5-min RR interval, where one value is gained for each HRV parameter per infant.

The same procedure is used to clean the original data, and add artificial artifacts into the artifact-free RR intervals, as described in section 4.2.2. For each infant, HRV parameters are calculated for the artifact-free RRs (HRV0), for the artificial artifact contaminated RRs (HRV1), and for the RRs that are cleaned using the newly developed artifact detection algorithm $\mathrm{f}$ (HRV2). The HRV changes from the artifact-free HRV in 
percentage, is used to compare the influence of the artifacts on the HRV measurements. The average changes over the 10 infants are used to do the comparison. The changes of $\mathrm{HRV}$ in percentage from the original is defined as:

$$
\text { Changes }(\%)=\frac{|H R V 1-H R V 0|}{H R V 0} \times 100 \%
$$

\subsubsection{Effect of artifacts on different HRV parameters}

Different numbers of artifact are added to the 5-min artifact-free real RR interval data in each of the 10 infants. Average changes in percentage of each HRV parameter across 10 infants are calculated for both noisy RRs and RRs cleaned by algorithm $\mathrm{f}$. Figure 4.7 and appendix C (Table 1) show the results. As expected, the effects of artifacts on HRV parameters are extremely variable. Median and interquartile range are better than mean and standard deviation in noisy situations. Frequency domain measurements are the most artifact affected parameters, and beat-to-beat variability are more likely to be affected by the artifacts. These results provide guidelines for choosing HRV parameters in clinical diagnosis or research in noisy environments.

After using the artifact detection algorithm $\mathrm{f}$, changes in HRV measurements are largely decreased. However, for some parameters, such as LF and HF power, there are still unacceptable changes (approximately 20\%). Since the two scattered missing beats are successfully detected and interpolated, the HRV changes might be the result of specificity and subsequent interpolation, as explored in the next section. 


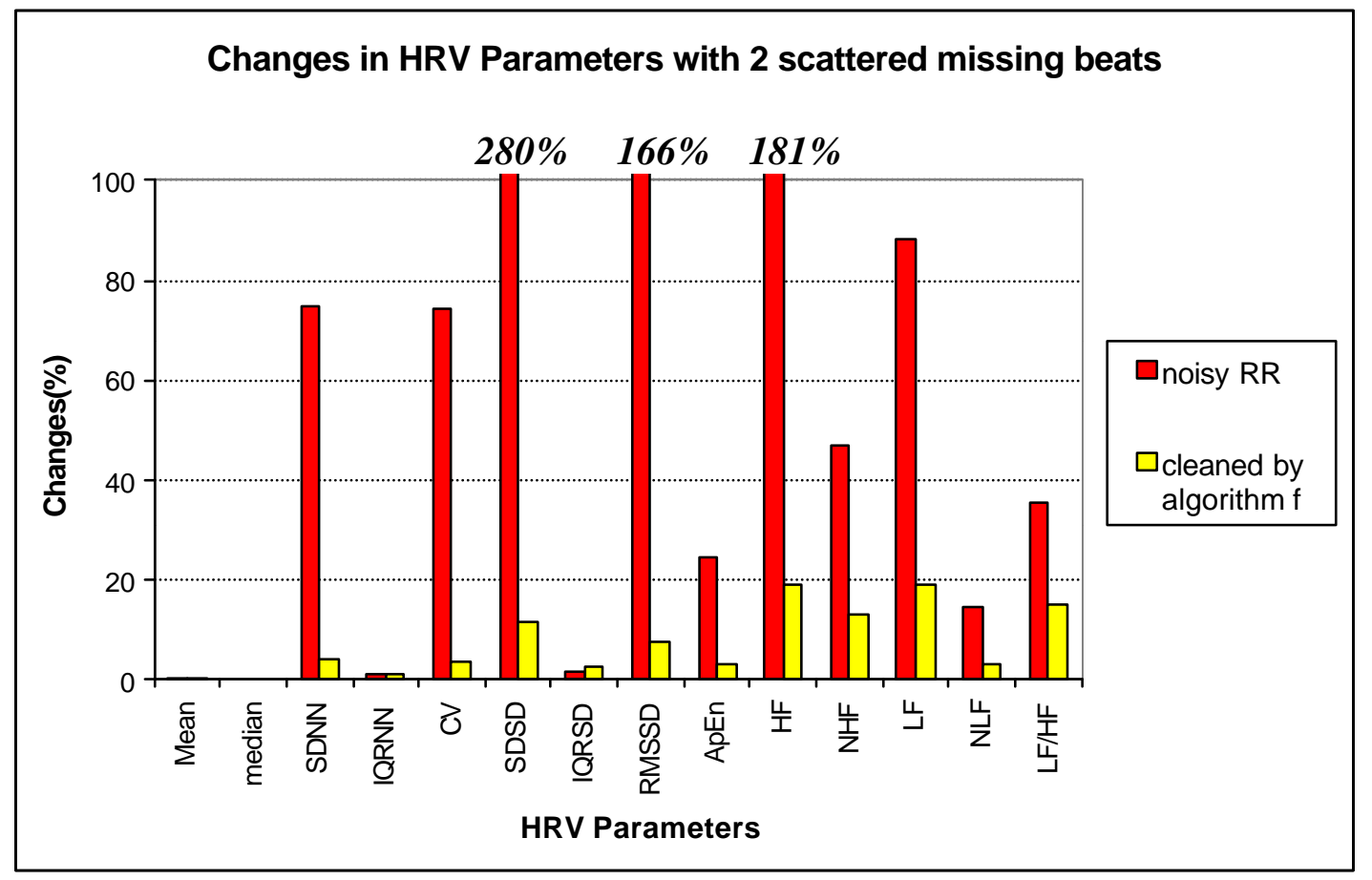

Figure 4.7 Effect of two scattered missing beats on HRV parameters

\subsubsection{Effect of specificity on HRV changes}

From the results in section 4.5.1, there are still unacceptable changes in some HRV parameters even after using the artifact removal routine. False positives of the algorithm may be implicated. Since the specificity of the algorithm $\mathrm{f}$ is not $100 \%$ for every infant, it detects several good beats as artifact, and replaces them with an interpolated value. Such changes in the RR interval time series affect the resultant HRV measurements.

To evaluate the effect of specificity on HRV changes, first, we apply the artifact removal routine on the same 5-min artifact-free RR intervals segments of the 10 infants. Second, we calculate the HRV parameters. Finally, the average changes in HRV from the original are evaluated. Figure 4.8 and appendix $C$ (Table 2) shows the result. Changes in 
HRV parameters from the original are only from false positives of the artifact detection algorithms. As expected, there are still changes in some HRV parameters, such as SDSD, RMSSD, HF, LF, and LF/HF. These changes also imply that specificity is as important as sensitivity of an artifact detection algorithm, as it can also affect the HRV measurements seriously.

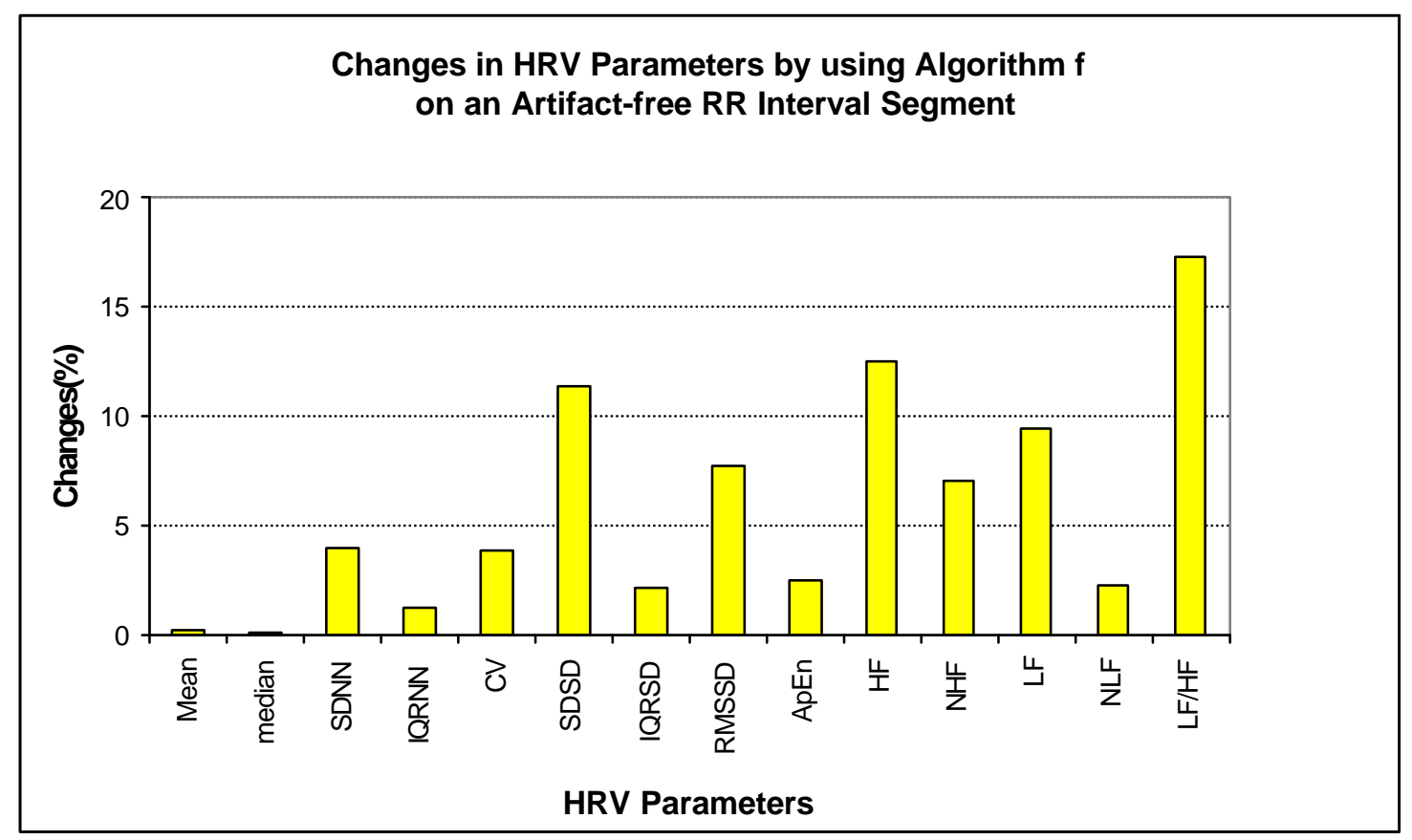

Figure 4.8 Effect of false positives of the artifact detection algorithm on HRV

\subsubsection{Effect of type $4 \& 5$ real artifacts on HRV changes}

As described in section 4.4.1, when dealing with real artifacts in our infant RR interval data, the sensitivity of the algorithms is comparatively lower. The major culprit for decreased sensitivity is type 4 and type 5 artifacts, noise where the QRS is discernable or undetectable, respectively. However, compared with missing beats and extra beats, it is hypothesized that these two types of artifact will have only a small effect on HRV 
measurements since the $R R$ interval values are near the real $R R$ interval. This section studies the effect of type 4 and type 5 artifacts on the HRV measurements.

Since we have already marked each type of artifact to form the dataset with real artifact, we use the same real artifact dataset for his study, which contains both 15-min randomly chosen and 15-min noisy RR interval segments from the 10 infants. There are totally 39982 beats in the twenty 15 -min RR interval segments. Among them, there are 14 type 4 and 463 type 5 artifacts.

For the 20 15-min RR interval segments, HRV parameters are calculated for three consecutive 5-min segments of each 15-min RR interval data, and then averaged. For each of the 5-min segments, first, we calculate the HRV parameters for the artifact-free RR intervals, which were cleaned by interpolation according to the beat classification by visually scanning the ECG. Second, all types of artifacts are removed other than type 4 and 5, and the HRV is calculated. In this case, the changes in HRV show the effect of only these two types of artifact on HRV. Third, we apply the artifact detection algorithm f on the RR interval data, and found the type $4 \& 5$ artifacts that are missed by the algorithm. Then all other artifacts are removed as marked by ECG scanning except the missed type $4 \& 5$ artifacts, and HRV is calculated. This allows us to investigate how the HRVs are affected by type $4 \& 5$ artifacts that are missed by artifact detection algorithm f. The average changes of the HRV from the artifact-free HRV in percentage, is used to compare the influence of the type $4 \& 5$ artifacts, and the missed type $4 \& 5$ artifacts by artifact detection routine. The averaged percentage changes over the 10 infants and all segments are used to do the comparison. 
The results are showed in figure 4.9 and appendix $\mathrm{C}$ (Table 3). Compared with the changes in HRV with two missing beats in each 5-min RR interval segment for the 10 infants (totally 20 missing beats), the 477 type $4 \& 5$ artifacts have comparatively less effect on the HRV measurements. The results on this real artifact dataset are consistent with those on artificial artifact dataset. The frequency domain and the $\Delta \mathrm{RR}$-based measurements are most sensitive to artifacts. However, the normalized HF and LF power are much better than the absolute HF and LF power under noisy situations. The 190 type 4 and type 5 artifacts that were missed by artifact detection algorithm $\mathrm{f}$ have little effect on the HRV calculation. The improvement of sensitivity and specificity of the artifact detection algorithm will improve the reliability of the HRV calculation.

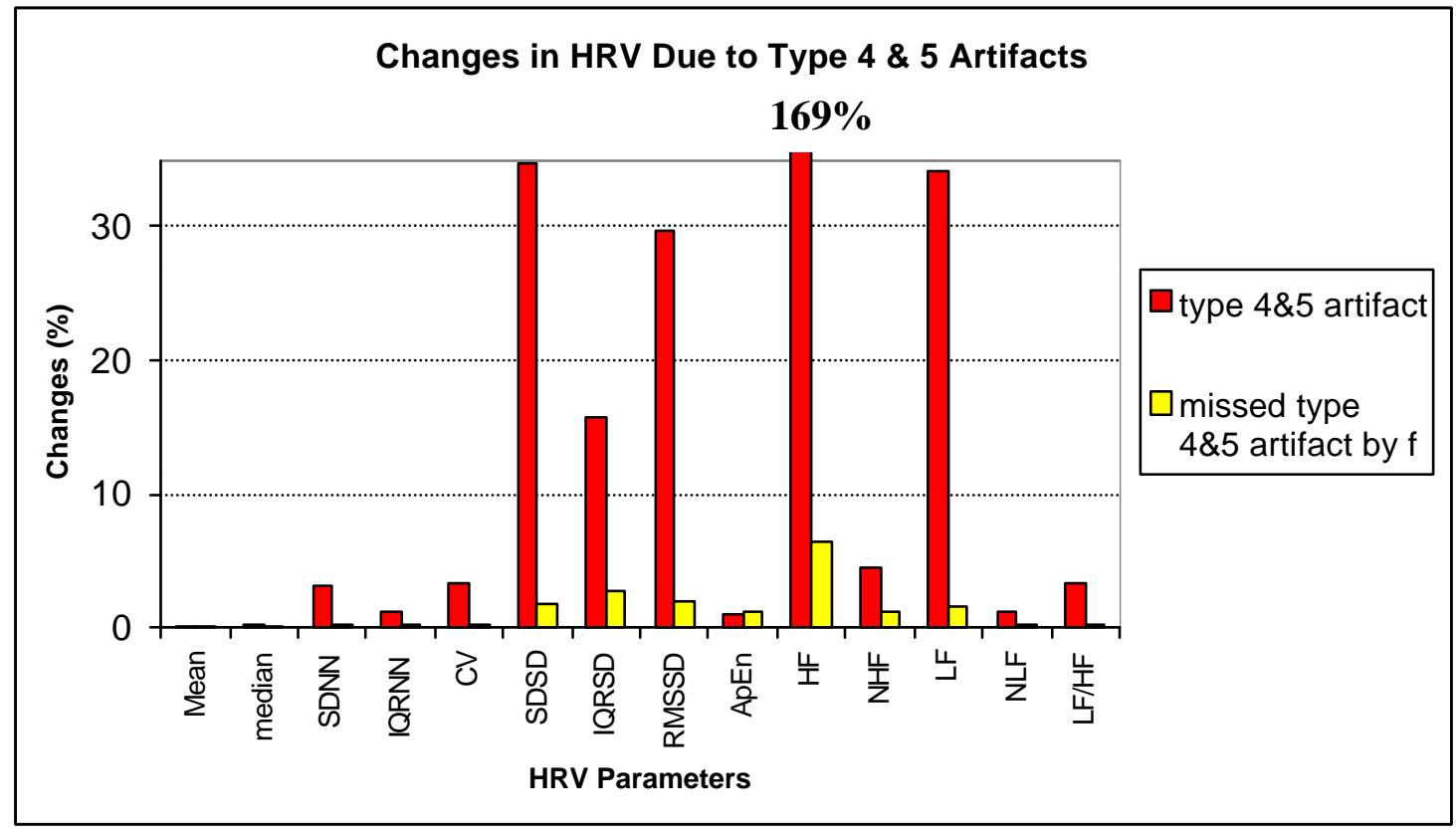

Figure 4.9 Effect of type $4 \& 5$ artifacts and algorithm $\mathrm{f}$ on HRV 


\subsection{Conclusion}

It is impractical to remove artifacts manually when dealing with a large dataset. Based on previous studies in this area, we developed a new automatic artifact detection algorithm. Ten infants' RR interval data are used to evaluate the algorithm by comparing it with five other algorithms. Both real artifacts in the infant RR interval and artificial artifacts are used to assess the performance of the algorithms. Sensitivity, specificity, and accuracy are used to quantify the performance. The newly developed algorithm performs better than other algorithms for detecting real artifacts in infant $R R$ interval data, providing the best balance between sensitivity and specificity $(73.46 \%$ and $99.17 \%$, respectively), greatly improving specificity with only a slight loss in sensitivity. It works extremely well for detecting missing and extra beats $(100 \%$ and $97.44 \%$ sensitivity, respectively) even on very noisy data. In terms of detecting artificial missing beats and extra beats, high sensitivity is achieved in most situations, and there is not much difference in specificity among the algorithms. In general, the newly developed algorithm provides the best overall performance, and offers acceptable detection accuracy.

Studying the effect of artifact on the heart rate variability measurements emphasizes the importance and effectiveness of the automatic artifact rejection routine. Even two missing beats in 5 minutes RR interval data will result in tremendous changes in some HRV parameters. Compared with missing beats and extra beats, type 4 and type 5 artifacts, noise where the QRS is discernable or undetectable, respectively, have comparatively less effect on HRV. In addition, although it is very difficult for the automatic artifact detection algorithms to detect type 4 and type 5 artifacts, these two 
types of artifact that were missed by our newly developed algorithm have only a small effect on the calculation of heart rate variability.

The testing results show that the effect of artifact on different HRV parameters can be quite different. Median and interquartile range are better than mean and standard deviation. Frequency domain measurements and beat-to-beat variability are the most sensitive parameters. These results also suggest using the HRV parameters less affected by artifact for noisy RR interval data.

The change in HRV from artifact free RR intervals provides another way to evaluate the effectiveness of the algorithm. Applying the newly developed artifact rejection routine largely decreases the change in $\mathrm{HRV}$, and the changes in most of the HRV parameters are acceptable. However, false positives of the algorithm resulted in non-ignorable HRV changes in some parameters. Extra caution must be taken when using those sensitive HRV parameters in clinical study.

Since the changes in HRV after applying the artifact rejection routine are mainly due to the false positives of the algorithm, this demonstrates the importance of improving the specificity of the automatic artifact detection algorithms. The lower specificity of several existing artifact identification algorithms makes them inappropriate for our study. The newly developed algorithm f shows its better characteristics, which provide the best balance between sensitivity and specificity. Compared with other previous algorithms, it greatly improves the specificity with a slight lose of sensitivity and offers the best overall performance in cleaning artifacts for our infant RR intervals. 


\title{
CHAPTER V \\ RELATIONSHIP OF HEART RATE VARIABILITY MEASURES
}

\begin{abstract}
Hypothesis: We hypothesize that many of the numerous HRV parameters which have been developed are correlated and this information can assist in infant classification.
\end{abstract}

Since the confirmation by many studies that heart rate variability is a strong and independent predictor of mortality after an acute myocardial infarction, the application of heart rate and heart rate variability is rapidly increasing in clinical studies. As we described in the previous chapters, there are many methods used in quantifying heart rate variability (HRV), including time-domain, frequency-domain, time-frequency-domain, and nonlinear methods. Generally, time-domain methods are ideal for the analysis of long-term recordings, while frequency-domain methods should be preferred to the time domain methods when short-term recordings are investigated. Frequency domain methods are also better than the time domain in separating parasympathetic and sympathetic influences and measuring the balance between sympathetic and parasympathetic systems. Compared with the frequency-domain methods, timefrequency-domain methods and wavelet work better for nonstationary signals, such as the $\mathrm{RR}$ interval, because it keeps the time information in the data. Since the RR interval involves nonlinear phenomena, nonlinear methods might provide additional information when characterizing them. Despite the variety of HRV measures, different measurements might have the same capability in predicting the risk of future apparent life-threatening events, or a combination of some HRV parameters might perform the best for the 
prediction. In this section of the dissertation, we perform a systematic investigation of the relationship of the most commonly used HRV measurements. Evaluation of their ability to predict apparent life-threatening events is described in next chapter.

\subsection{Introduction}

Time-domain, frequency-domain, time-frequency-domain, and nonlinear measures of HRV have been used to assess the function of the autonomic nervous system and to predict risk of death or arrhythmia in patients with cardiovascular diseases. They all have their own advantages and disadvantages. Bigger et al. suggested that timedomain and frequency-domain HRV measures provide equivalent information in predicting mortality [BIG92]. Actually, few studies have been done to determine the relationship between these HRV measurements. In addition, it is not clear which measurements may be quantifying the same underlying feature in heart rate. Thus, researchers or clinicians might have difficulties in choosing the appropriate method for their application and dataset. Studying the relationship among these HRV parameters is meaningful and might lead to a proper selection of HRV or HRV groups to predict SIDS.

It is widely accepted that pNN50, RMSSD, and HF form one highly correlated group. These HRV parameters were even thought to be interchangeable. Twenty-fourhour ECG from 857 survivors after acute myocardial infarction were used to study the relationship between RMSSD and pNN50 in [TASK96]. Scatter plot was used to show the relationship between those parameters. They concluded that these three HRV parameters measure the high frequency variation in heart rate, so they are highly correlated. Bigger et al. also tried to determine the strength of association among the 
same three HRV parameters. They expected strong correlations among them, and hoped the relationship would be strong enough that they are interchangeable for assessing vagal activity. They also expected weaker correlation of these three measures with the total power and SDNN. Spearman's correlation coefficients rather than Pearson's correlation coefficient were used to quantify the strength of association. The results of their study met their expectations [BIG89]. Study in [KLE91, SPI93] also confirmed these results. They concluded that RMSSD and pNN50, especially RMSSD, can be used as surrogates for $\mathrm{HF}$, since time domain variables are inexpensive to compute compared with frequency domain variables.

Some controversial results were reached in the study of the correlation between other HRV parameters also. Weak correlation was found between SDNN with HF in [KLE91]. However, they find that LF over 24 hours is highly correlated with RMSSD, $\mathrm{HF}$, and pNN50, and total power over 24 hours is also strongly correlated with LF, HF, RMSSD, pNN50, and SDNN in their studied subjects. These results indicate that total power and LF over 24 hours are vagal dependent parameters in their subjects. However, most other researches believe that LF is dependent on sympathetic tone rather than vagal tone, and total power is highly correlated with SDNN [BIG89, BIG92, COS94].

24-hour Holter recordings of nine normal adults were used to do a comparative study of HRV parameters in time and frequency domain in [COS94]. They also compared the long-series and short-series HRV. The long-series HRV are defined as HRV parameters that are calculated from 24 nonoverlapping 1-hour segments. While, the short-series HRV are HRV measures that computed from 512 beats extracted every hour. Most of the time the first 512 beats is selected if there are no abrupt changes in heart rate. 
The Pearson's correlation coefficient $\gamma$ and Spearman's correlation coefficient $\rho$ were used to measure the relationship. They found strong correlation between LF and SDNN, and between HF and pNN50. There was no significant difference between long-series and short-series HRV in calculating the correlation coefficients.

Myers et al. studied the correlation between the frequency parameters (four frequency components) of HRV and four time-domain HRV indexes (SDNNIDX, SDANN, SDNN, and pNN50) using a 24 hour ECG of 18 subjects. They also compared the efficiency of these parameters in predicting the high risk group of sudden cardiac death (SCD) in adults. Their results also confirmed that pNN50 represents high frequency variance in heart rate, and it is highly correlated to high power frequency components $\mathrm{C}$ and D (C: $0.15-0.35 \mathrm{~Hz}, \mathrm{D}: 0.35-0.50 \mathrm{~Hz})$, and to a lesser extent, to component $\mathrm{B}(0.05-$ $0.15 \mathrm{~Hz}$ ). SDANN operates like a low-pass filter, and is sensitive to component A $(0.0167-0.05 \mathrm{~Hz})$ and to the posture changes and activities that greatly affect mean heart rate. The SDNNIDX may be represented as a high pass filter in the Fourier domain. It is sensitive to B-D components. They found that frequency components $\mathrm{C}$ and $\mathrm{D}$ are the best index in separating the SCD and non-SCD groups [MYE86].

The approximate relationship between time and frequency domain HRV parameters measured from 24-hour ECG recordings was summarized in [TASK96]. The relationship is shown in the following table. They believed that from both mathematical and physiological points of view, the strong correlation between the time and frequency domain HRV parameters in the table can be explained. 
Approximate Correspondence of Time and Frequency Domain Methods

Applied to 24-hour ECG Recordings [TASK96]

\begin{tabular}{ll}
\hline Time Domain Variable & $\begin{array}{c}\text { Approximate Frequency } \\
\text { Domain Correlate }\end{array}$ \\
\hline SDNN & Total power \\
HRV triangular index & Total power \\
TINN & Total power \\
SDANN & ULF \\
SDNNIDX & Mean of 5-min total power \\
RMSSD & HF \\
SDSD & HF \\
Count & HF \\
pNN50 & HF \\
\end{tabular}

Yeragani et al. compared nonlinear measures of HRV, Fractal Dimension (FD) and Approximate Entropy (ApEn), using 24 hours of ECG from twenty-three normal subjects. The correlation was studied for different sleep states. The highly correlated groups include ApEn with FD, HF with FD, HF with ApEn, and mean RR interval with ApEn, for both awake and sleep. Significant correlation between FD and mean RR interval was only found in awake [YER98].

In general, all HRV parameters described in chapter 3 have been used in many clinical or diagnostic studies. However, the relationship between these HRV measures has never been studied systematically. This relationship should be investigated thoroughly, such that HRV can be used more efficiently and the results can be correctly and consistently interpreted.

There are differences between our study and previous studies. Previous studies of the relationship among HRV measurements were performed on adult data, and few considered sleep state [MYE86, BIG89, KLE91, SPI93, COS94, TASK96, YER98]. In addition, other studies used the mean or median of HRV parameters over a long period of 
time for each subject to calculate the correlation coefficients, and did not include as many HRV parameters. We use a series of 5-min HRV measures per infant per sleep state for the correlation study. This will also take into account the variance in each sleep state per infant. We study the relationship of every combination of the $24 \mathrm{HRV}$ parameters.

Furthermore, our ultimate goal is to classify or predict infant group by HRV with regard to apparent life-threatening events. Besides finding the best model to classify the infant group accurately, it is also very important to determine the most efficient design of the model for our specific task, that is, the minimum number of input variables which will result in an acceptable prediction precision. Since HRV are the major input variables, this process will be assisted by studying the relationship between HRV parameters. By choosing one or two HRV parameters from each highly correlated group as the input variables, we can simplify the structure of the model and compare the results of these different models. In general, the information from this aspect of the research can help us determine which parameters to use in our classification methods to minimize the number of inputs and the effect of noise on the results.

\subsection{Material and Methods}

There are $24 \mathrm{HRV}$ parameters included in this correlation study: Mean, Median, SDNN, IQRNN, NIQRNN, CV, SDSD, IQRSD, NIQRSD, RMSSD, CVS, pNN50, HF, LF, NHF, NLF, total power, LF/HF, HFW, LFW, LFW/HFW, ApEn, Poincare, and Fractal Dimension. Scatter plots and Pearson's correlation coefficients are used to view and measure the relationship between HRV parameters in this study. Since the calculation of HRV parameters is influenced by sleep state and since the infants are from 
different patient populations, we are also able to evaluate whether the relationship is affected by sleep state and group differences. Therefore, within each sleep state, correlation coefficients will be calculated for each of the infants, for all of the infants, and for each group of preterm, ALTE, SIDS siblings, and healthy infants. A 5-number summary and standard deviation will be used to summarize the results.

Different from other correlation studies that used one averaged HRV value over the entire long-term recording for each sleep state per infant, we use the HRV values of all 5-min segments for the calculation of correlation coefficients. As described in chapter 3, heart rate variability parameters are calculated for 5-min segments over the whole 8hour RR interval data. Therefore, for one HRV parameter, a vector of 5-min measurements for each sleep state and infant will be used to calculate the correlation coefficients. A certain number of 5-min segments in each sleep state is required for further analysis. We plan to use infants who have at least five 5-min segments for each of the three sleep states.

We pursue two different methods for calculating the overall correlation. The first is to calculate correlation coefficients for each infant. The second is to generate a composite correlation across all infants. Because different infants have a different number of 5-min segments in each sleep state, a bootstrap method will be used to get an accurate measure of correlation. The idea of the bootstrap method is to use the same amount of data from each infant in the correlation study, such that the infants have the same contribution to the final result. 


\subsubsection{Correlation coefficient}

The relationship between two variables, $\mathrm{X}$ and $\mathrm{Y}$, is a general subject of study. Scatter plot, a plot of the values of $\mathrm{Y}$ versus the corresponding values of $\mathrm{X}$, gives a graphical representation of the relationship or association between two variables. It is always constructed when the relationship of two variables is of interest.

The correlation between two variables quantitatively reflects the degree to which the variables are related. There are several important kinds of correlation, such as Pearson's, Spearman's, and intraclass correlation coefficients. The details of calculation for them are different. Among them, the most common used measurement of correlation is the Pearson Product Moment Correlation, which was shortened as Pearson's correlation coefficient. Pearson's correlation coefficient is a single number that used to measure the strength of linear relationship between two variables $\mathrm{X}$ and $\mathrm{Y}$, and it is usually signified by $r$. The definition of the Pearson's correlation coefficient is:

$$
r=\frac{\operatorname{Cov}(x, y)}{\sigma_{x} \sigma_{y}}=\frac{\sum_{i}\left(x_{i}-\bar{x}\right)\left(y_{i}-\bar{y}\right)}{\sqrt{\left(\sum_{i}\left(x_{i}-\bar{x}\right)^{2}\right)\left(\sum_{i}\left(y_{i}-\bar{y}\right)^{2}\right)}}
$$

$r$ can take on the values from -1.0 to 1.0 , where -1.0 is a perfect negative (inverse) correlation, 0.0 is no correlation, and 1.0 is a perfect positive correlation.

Since the values of the Pearson's, Spearman's, and intraclass correlation coefficients are usually similar for the same set of data, we decided to choose only the Pearson's correlation coefficient for our study. 


\subsubsection{Correlation from individuals}

The variance among individuals in heart rate and heart rate variability is obvious. In order to determine the consistency of the relationships between HRV parameters across infants, the correlation coefficients are calculated for each infant with sleep state taken into consideration first. Then a 5-number summary and standard deviation are used to summarize the results.

A 5-number summary is a very concise way to describe the major features of a set of results without getting into the details. Definition of the 5 numbers varies slightly from study to study, such as $10^{\text {th }}, 25^{\text {th }}, 50^{\text {th }}, 75^{\text {th }}$ and $90^{\text {th }}$ percentiles, or minimum, $25^{\text {th }}, 50^{\text {th }}$, $75^{\text {th }}$ percentiles and maximum. We define the 5 -number summary here as $2.5^{\text {th }}, 25^{\text {th }}, 50^{\text {th }}$, $75^{\text {th }}, 97.5^{\text {th }}$ percentile of the coefficients in our study. The $50^{\text {th }}$ percentile is the median, and the $25^{\text {th }}$ and $75^{\text {th }}$ percentiles are called quartiles. The distance between the quartiles and the Median shows how spread out the distribution of the result is. Generally, these five numbers together give a very informative description of the set of results, without distraction by too many details.

\subsubsection{Overall correlation}

It is also appropriate to study the correlation for the whole population. Different from other correlation studies that used one averaged HRV value over the entire longterm recording for each sleep state per infant, we use the HRV values of all 5-min segments for the calculation of correlation coefficients. Therefore, the HRV parameters

over 8 hours for the 110 infants are gathered together. Since different infants have a different number of 5-min segments in each sleep state, a bootstrap method will be used 
to get an accurate measure of correlation, while equally weighting the contribution from each infant.

The procedure for using the bootstrap method is as follows: 1) find the minimum number of 5-min segments among all infants for each sleep state (Nqs, Nrem, Nawk); 2) randomly choose Nqs/Nrem/Nawk segments from all other infants in QS/REM/AWK to form a bootstrap sample set; 3) calculate the sample correlation based on this bootstrap sample; and 4) repeat step 2 and 3 a large number of times, i.e. 1000 times, to obtain 1000 bootstrap replicas of the correlation coefficients. The mean, median, or other statistical measurements of these replicas can be used as an accurate estimation of the true correlation. Taking the 25 th and 97.5th largest of these 1000 replicates can give us the rough $95 \%$ confidence interval. In this method, the same number of segments from each infant will be used in the calculation of correlation coefficients, so the contribution of each infant to the correlation is the same. Furthermore, the same 5-number summary and standard deviation are used to summarize the results.

\subsubsection{Overall correlation with infant group taken into consideration}

The infants in our study were originally categorized into four groups, healthy, preterm, SIDS siblings and infant with apparent life-threatening events (ALTE). This additional information allows us to investigate whether the correlation between HRV parameters varies with regard to group differences. The same bootstrap method is used to calculate the overall correlation coefficient for the four groups of infants. 
There are 15 healthy, 54 preterm, 19 SIDS siblings, and 22 ALTE infants forming the above groups, respectively. The correlation coefficients are calculated based on the HRV parameters of these infants respectively.

\subsection{Results and Discussion}

Whether the correlation study is performed on individual infants or on the bootstrap sample set of the whole infant group, there is a large number of correlation coefficients for each pair of HRV parameters, eg. a correlation coefficient for each infant, or 1000 bootstrap replicas of the correlation coefficient. A 5-number summary and standard deviation is used to summarize the results. Therefore, for each pair of HRV parameters, there are five 5-point summaries for each sleep state, which are gained from individual coefficients, bootstrap coefficients of healthy, preterm, SIDS siblings, ALTE, and all of the infants, respectively. These results and corresponding scatter plots are used to evaluate the correlations.

Tables 5.1 and 5.2 use the median of the correlation coefficients for the all infants' bootstrap dataset in QS and AWK, respectively, to demonstrate the overall results. The colors visually represent the level of relationships between all pairs of HRV parameters. Red and orange indicate the most correlated pair of parameters. In addition the correlation coefficients in AWK are generally lower than corresponding ones in QS. Detailed results are in appendix D. 
Table 5.1 Median of the correlation coefficients of the all infants' bootstrap dataset in QS

\begin{tabular}{|c|c|c|c|c|c|c|c|c|c|c|c|c|c|c|c|}
\hline HRVs & Mean & SDNN & IQRNN & IQRSD & RMSSD & $\mathrm{HF}$ & LF & Tpower & LF/HF & HFW & LFW & LFW/HFW & ApEn & Poincare & FD \\
\hline Mean & 1.0000 & 0.6051 & 0.6273 & 0.7198 & 0.7372 & 0.6458 & 0.4084 & 0.5187 & -0.2316 & 0.6631 & 0.4537 & -0.2738 & -0.1475 & 0.6700 & 0.5691 \\
\hline SDNN & & 1.0000 & 0.9318 & 0.7074 & 0.7405 & 0.6536 & 0.7506 & 0.8026 & -0.0006 & 0.7029 & 0.8515 & 0.0017 & -0.4824 & 0.9281 & 0.6389 \\
\hline IQRNN & & & 1.0000 & 0.7499 & 0.7686 & 0.6837 & 0.7162 & 0.7839 & -0.0349 & 0.7280 & 0.8116 & -0.0634 & -0.3373 & 0.9838 & 0.6817 \\
\hline IQRSD & & & & 1.0000 & 0.9767 & 0.8610 & 0.4753 & 0.6373 & -0.3376 & 0.8831 & 0.5462 & -0.3868 & 0.0919 & 0.8205 & 0.9269 \\
\hline RMSSD & & & & & 1.0000 & 0.8881 & 0.5013 & 0.6657 & -0.3325 & 0.9117 & 0.5721 & -0.3806 & 0.0283 & 0.8394 & 0.9347 \\
\hline $\mathrm{HF}$ & & & & & & 1.0000 & 0.5156 & 0.7095 & -0.2437 & 0.9672 & 0.5341 & -0.2879 & -0.0299 & 0.7496 & 0.7522 \\
\hline LF & & & & & & & 1.0000 & 0.9701 & 0.1700 & 0.5065 & 0.8390 & 0.0786 & -0.3337 & 0.6971 & 0.3948 \\
\hline Tpower & & & & & & & & 1.0000 & 0.0702 & 0.6915 & 0.8415 & -0.0189 & -0.2845 & 0.7856 & 0.5388 \\
\hline $\mathrm{LF} / \mathrm{HF}$ & & & & & & & & & 1.0000 & -0.2432 & 0.0836 & 0.7821 & -0.4722 & 016 & -0.3961 \\
\hline HFW & & & & & & & & & & 1.0000 & 0.5890 & -0.2854 & -0.0565 & 0.7945 & 0.7714 \\
\hline LFW & & & & & & & & & & & 1.0000 & 0.1275 & -0.3889 & 0.7891 & 0.4471 \\
\hline LFW/HFW & & & & & & & & & & & & 1.0000 & -0.5466 & -0.1367 & -0.4519 \\
\hline ApEn & & & & & & & & & & & & & 1.0000 & -0.2769 & 0.1782 \\
\hline Poincare & & & & & & & & & & & & & & 1.0000 & 0.7511 \\
\hline FD & & & & & & & & & & & & & & & 1.0000 \\
\hline
\end{tabular}

Table 5.2 Median of the correlation coefficients of the all infants' bootstrap dataset in AWK

\begin{tabular}{|c|c|c|c|c|c|c|c|c|c|c|c|c|c|c|c|}
\hline HRVs & Mean & SDNN & IQRNN & IQRSD & RMSSD & $\mathrm{HF}$ & LF & Tpower & LF/HF & HFW & LFW & LFW/HFW & ApEn & Poincare & FD \\
\hline Mean & 1.0000 & 0.2941 & 0.3136 & 0.4361 & 0.3780 & 0.3237 & 0.2301 & 0.2439 & 0.0652 & 0.4183 & 0.3131 & 0.0455 & $0.094 c$ & 0.2945 & 0.0512 \\
\hline SDNN & & 1.0000 & 0.7502 & 0.3858 & 0.5446 & 0.4030 & 0.5571 & 0.5668 & 0.3198 & 0.5448 & 0.6822 & 0.1556 & $-0.442 \varepsilon$ & 0.8249 & 0.3341 \\
\hline IQRNN & & & 1.0000 & 0.3908 & 0.3677 & 0.3007 & 0.2472 & 0.2596 & 0.0268 & 0.3586 & 0.3214 & 0.0163 & -0.3331 & 0.9744 & 0.2888 \\
\hline IQRSD & & & & 1.0000 & 0.8545 & 0.6260 & 0.2206 & 0.2664 & -0.2323 & 0.6960 & 0.3004 & -0.3063 & 0.4863 & 0.4023 & 0.8522 \\
\hline RMSSD & & & & & 1.0000 & 0.7428 & 0.4043 & 0.4317 & -0.1829 & 0.8644 & 0.4882 & -0.3326 & $0.285 \varepsilon$ & 0.4345 & 0.8617 \\
\hline $\mathrm{HF}$ & & & & & & 1.0000 & 0.5275 & 0.6021 & -0.1845 & 0.8264 & 0.4113 & -0.2313 & $0.125 \varepsilon$ & 0.3242 & 0.5755 \\
\hline LF & & & & & & & 1.0000 & 0.9973 & 0.3247 & 0.5038 & 0.7343 & 0.1768 & -0.1980 & 0.3627 & 0.1703 \\
\hline Tpower & & & & & & & & 1.0000 & 0.2824 & 0.5543 & 0.7356 & 0.1422 & $-0.173 \varsigma$ & 0.3755 & 0.2113 \\
\hline LF/HF & & & & & & & & & 1.0000 & -0.1384 & 0.2772 & 0.6488 & $-0.382 \varepsilon$ & 0.0893 & -0.3400 \\
\hline HFW & & & & & & & & & & 1.0000 & 0.6099 & -0.2077 & 0.070 & 0.4252 & 0.6140 \\
\hline LFW & & & & & & & & & & & 1.0000 & 0.3246 & -0.2645 & 0.4435 & 0.2150 \\
\hline LFW/HFW & & & & & & & & & & & & 1.0000 & -0.459 & 0.0513 & -0.4293 \\
\hline ApEn & & & & & & & & & & & & & 1.000 & -0.3572 & 0.4792 \\
\hline Poincare & & & & & & & & & & & & & & 1.0000 & 0.3167 \\
\hline FD & & & & & & & & & & & & & & & 1.0000 \\
\hline
\end{tabular}

\begin{tabular}{|c|c|c|c|c|c|c|c|c|c|c|}
\hline 1 & $0.9 \sim 1.0$ & $0.84 \sim 0.9$ & $0.75 \sim 0.84$ & $0.65 \sim 0.75$ & $\begin{array}{c}\mathbf{0 . 5 5} \sim 0.65 \\
105\end{array}$ & $0.1 \sim 0.55$ & $0 \sim 0.1$ & $-0.1 \sim-0.2$ & $-0.2 \sim-0.3$ & $-0.3 \sim-0.45$ \\
\hline
\end{tabular}


The bar chart in figure 5.1 shows the median correlation coefficients between selections of HRV pairs in quiet sleep. The correlation is calculated from individual infants, bootstrap dataset of all infants and healthy, ALTE, sibling, and preterm infants. The differences among the results from different group of infants are not significant.

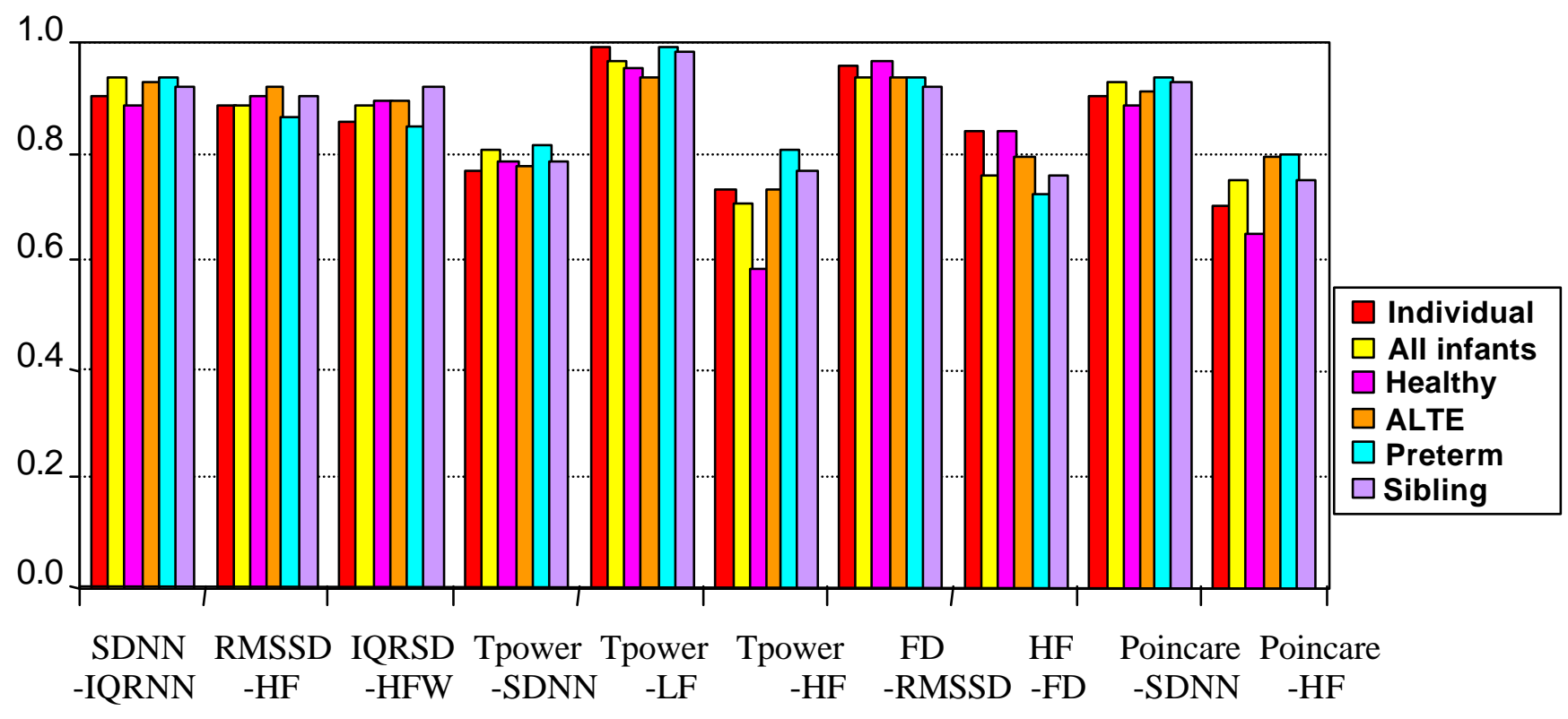

Figure 5.1 Median of correlation coefficients for QS

After obtaining the correlation coefficients and getting a general idea of the correlation, rules for both the level of correlation and consistency across infants are needed to determine some conclusions regarding correlation of HRV. There are four levels of correlations defined here for this study, very strong correlated, highly correlated, somewhat correlated, and not correlated. (1) For the very strong correlated pairs, the threshold is set as: the median of the correlation coefficients is not less than 0.95, and the standard deviation is not larger than 0.06. (2) For the highly correlated pair: the median of the correlation coefficients is not less than 0.80 , and the standard deviation is not larger than 0.2. (3) For the somewhat correlated group: the median of the correlation 
coefficients is ot less than 0.5 , and the standard deviation is not larger than 0.3. (4) The rest of them are defined as the not correlated group.

\subsubsection{Very strong correlations}

Among these $24 \mathrm{HRV}$ parameters, some of the HRV pairs should be very strongly correlated according to their definition. The results from our infant data confirms this. The strong correlated HRV pairs confirmed by our study include Mean \& Median, SDNN \& CV, IQRNN \& NIQRNN, IQRSD \& NIQRSD, RMSSD \& CVS \&SDSD, and NHF \& NLF (negative correlation). Table 5.1 in appendix D shows how these pairs of HRVs are strongly correlated. From the 5-number summary, the range of the correlation coefficients for Mean and Median is from 0.9009 to 0.9994 , from 0.9105 to 0.9993 for SDNN and CV, from 0.9456 to 0.9996 for IQRNN and NIQRNN, from 0.8616 to 0.9996 for IQRSD and NIQRSD, from 0.8498 to 0.9995 for RMSSD and CVS, and from 0.8165 to 0.9978 for RMSSD and SDSD. NHF and NLF are exactly negatively correlated. In addition, these strongly correlated HRV pairs have similar correlation with all other HRV parameters. Therefore, these HRV pairs can be considered as interchangeable. For better statistical characteristics, we choose Median, SDNN, IQRNN, IQRSD, and RMSSD rather than their peers for future analysis.

SDNN and IQRNN should also be strongly correlated according to their definition. Our results showed that the level of correlation is high, but the consistency across individual infants is not satisfied. By our rules, they belong to the highly correlated group. 


\subsubsection{A highly correlated group}

A widely accepted highly correlated group of HRVs that measures the vagal input include: SDSD, IQRSD, NIQRSD, RMSSD, CVS, pNN50, HF, NHF, and HFW. Many studies support this result. Table 5.2 in appendix D gives the results from our infant data. In the previous section, RMSSD was shown to be strongly correlated with SDSD, CVS. Due to its robustness to noise, we use RMSSD as a standard time domain measurement of the beat-to-beat variability in $\mathrm{RR}$ interval. The remaining parameters in this group, IQRSD, pNN50, HF, NHF, and HFW, are compared with RMSSD. Our results also confirmed the highly correlated relationship among these parameters, except pNN50, NHF and HF for REM and AWK sleep states. Even thought it does not meet the highly correlated criteria, the correlation coefficients between HF and RMSSD for REM/AWK are still high, ranging from 0.7238 to 0.9184 . Another exception is between SDSD and IQRSD for the AWK ( $\mathrm{r}=0.6468 \sim 0.8625)$, even though they are highly correlated for both QS and REM.

The results also show that pNN50 is not a good parameter for our infant RR interval data. Its correlation with other related parameters varies extensively from infant to infant, although the bootstrap correlation coefficients are large. NHF, the normalized high frequency power, is expected to be highly correlated with other HRV parameters that measure the high frequency component or beat-to-beat variation of RR interval. This is not the case in our study. Table 5.3 in appendix D gives the correlation coefficients of NHF with other HRV parameters in this group. Since the rest of the parameters in this group are all highly correlated, and are understood to clearly measure high frequency components, the NHF is not measuring the same thing as it is designed to be. In another 
words, the method which normalization is performed might not be the proper procedure. Therefore, we recommend not using pNN50, NHF and NLF for further analysis.

Consistent with many previous studies, our study confirmed a highly correlated group of HRVs, which include SDSD, IQRSD, NIQRSD, RMSSD, CVS, HF, and HFW. Among them, RMSSD is a better surrogate for other parameters because of its better statistical characteristics and lower computational expense.

\subsubsection{Conflicting results}

Some of our results conflict with those reached in previous studies of the relationship between frequency and time domain measurements. Total power is supposed to be highly correlated with SDNN. Furthermore, compared with HF, the LF is the dominant component in total power for most of our infants. Therefore, LF should also be highly correlated with total power and SDNN. Our results in table 5.4 of appendix D indicate that the total power is very strongly correlated with LF, which confirms previous results [KLE91]. However, the following pairs are only somewhat correlated: total power and SDNN for REM and AWK, LF and SDNN, total power and HF, and total power with RMSSD, while the study in [KLE91, COS94] found high correlation between them. In addition, we also found weak correlation between SDNN with HF for REM and AWK, as [KLE91] did.

In terms of whether LF is correlated with RMSSD and HF, most studies resulted in a negative answer, although high correlation between them is surprisingly founded in one study [KLE91]. From our result, we do not find high correlation between them. Another very important frequency domain HRV parameter is LF/HF. Our results do not 
show any relationship between LF/HF and any other HRV parameters. Furthermore, none of the frequency parameters is highly correlated with Median.

The frequency domain HRV parameters are calculated by both Fourier transforms and wavelet transform. It is important to evaluate whether the results are comparable or possibly even, whether they measure the same underlying feature. The relationships of corresponding parameters (HF with HFW, LF with LFW, and LF/HF with LFW/HFW) are studied here in this section. The results in table 5.5 of appendix D shows that HF is highly correlated with HFW, while the relationship of LF and LF/HF with LFW and LFW/HFW, respectively is very close to highly correlated relationship ( $\mathrm{r}=0.7736$, and 0.7529 respectively).

Another important group of HRV parameters are the nonlinear measurements of HRV, ApEn, Poincare and fractal dimension included in this study. The relationships between these nonlinear parameters have rarely been studied. Table 5.6 in appendix D summarizes the result of correlation among the three nonlinear parameters, and with other HRV parameters. No high correlation was found in our results between HF and FD, $\mathrm{HF}$ and ApEn, and ApEn and FD, as addressed in [YER98]. FD and HF are only somewhat correlated. In addition, there is not much correlation among any of the three nonlinear parameters, although Poincare with fractal dimension at QS which was somewhat correlated.

ApEn is not related with any other HRV parameters. Poincare is highly correlated with SDNN, strongly correlated with IQRNN, and somewhat correlated with RMSSD, HF, LF, and total power for QS sleep. While, FD is highly correlated with RMSSD, 
somewhat correlated with HF in all sleep states. Somewhat correlations are also found between FD with SDNN, IQRNN and Median for only QS sleep state.

\subsection{Summary}

In this chapter of the dissertation, the relationship between the most popularly used HRV parameters are studied systematically. Four levels of correlation are defined, very strongly correlated, highly correlated, somewhat correlated, and no correlation. According to the 5-number summary of individual infants, and bootstrap sample sets from all infants, healthy, siblings, ALTE, and preterm infant groups, we generally summarize the correlation between these HRV parameters as follows:

The very strong correlated group contains: Mean \& Median, SDNN \& CV, IQRNN \& NIQRNN, IQRSD \& NIQRSD, RMSSD \& CVS, RMSSD \& SDSD, and HF \& HFW. The correlation between these pairs can also be shown by their definition. Very strong correlation is also founded in total power \& LF, which confirms a previous study, and Poincare \& IQRNN.

The highly correlated group contains the HRV groups that measures vagal activity, which include SDSD, IQRSD, NIQRSD, RMSSD, CVS, HF, and HFW. They are all highly correlated with each other. Other highly correlated pairs include SDNN \& IQRNN, Poincare \& SDNN, and FD \& RMSSD.

The somewhat correlated group contains: total power \& SDNN, LF \& SDNN, total power \& HF, total power \& RMSSD, LF \& HF, SDSD \& IQRSD in AWK, and FD \& HF, which conflicts with one previous study. Measurements which were found to be 
somewhat correlated also include SDNN \& RMSSD, Poincare \& RMSSD in QS\&REM, Poincare \& HF/LF/total power in QS, Poincare \& FD in QS, FD \& SDNN/IQRNN in QS.

Some groups which were not found to be correlated include: LF \& RMSSD, HF \& SDNN in REM and AWK, ApEn \& FD, ApEn \& HF, FD \& Median for AWK, ApEn \& Median, and HF \& NHF.

While, there are some differences in correlation coefficients between HRV parameters for different sleep states, consistent results are reached for most of the correlations. In addition, the group difference does not change the relationship significantly. 


\section{CHAPTER VI \\ PREDICTION OF SUBSEQUENT LIFE-THREATENING EVENTS IN INFANTS USING HEART RATE VARIABILITY}

Hypothesis: We hypothesize that the heart rate variability in normal healthy infants is higher than for those infants who will go on to have serious life threatening events, such as apnea or bradycardia. These differences will allow us to classify these two types of infants.

The long-term objective of the study for this dissertation is to help the prevention of sudden infant death syndrome. It is widely accepted that decreased heart rate variability is often associated with diseases (coronary artery disease, SIDS etc.) and aging. Since HRV is under the control of the autonomic nervous system, the immaturity of the autonomic nervous system in an infant might be associated with the risk to SIDS or future apparent life-threatening events. Therefore, the major work of this dissertation is to determine if it is possible to classify infants by HRV parameters with regard to future apparent life-threatening events (FALTE). Our hypothesis is that the heart rate variability in normal healthy infants is higher than for those infants who will go on to have serious life threatening events, such as apnea or bradycardia. We hypothesize that these differences will allow us to classify infants through sophisticated pattern recognition techniques.

This section of the dissertation is to test the above hypothesis by investigating whether HRV can differentiate infants who go on to have life-threatening events from data collected from a polysomnographic (PSG) study. First we determine if there are statistical differences in heart rate variability between high risk infants and the controls. 
A two-sample ttest has been performed and described in Chapter 3. The results from the t-test will help us select inputs for classification. Second, we developed a predictive model based mainly on HRV parameters to predict which infants will go on to have events. Many different approaches have been used for solving the classification problem. For a classification or prediction problem like ours, which is supervised and has a dichotomous output, the most widely used methods include logistic regression, decision trees, and neural networks. The first section of this Chapter is an overview of the classification models. The second section is the methods for testing three models on the CHIME dataset. The third section is the results of our test for all the models. Lastly, in sections 6.4 and 6.5, the relationship of the HRV parameters studied in Chapter 5 and other information which affects classification will be used to simplify our predictive model and achieve the best model design.

\subsection{Basic Concept of Classification Models}

One of the major topics of this dissertation is to research ways to classify or predict infant groups with regard to future life threatening events. The basic concept of classification, supervised classification models, and the practical application for our specific infant classification problem is discussed in this section.

Classifying entities (observations) into one of several well-defined mutually exclusive groups is termed discriminant analysis (DA), or classification. It is an important problem in many applications. The classification is based on the characteristics of a set of relevant attributes, which are called independent (input) variables. While, the resultant group information is the response (output) variable. For our study, the heart rate 
parameters are the main input variables and whether there will be future life-threatening events is the response variable.

Many different approaches have been used for solving the classification problem. For a classification or prediction problem like ours, which is supervised and has a dichotomous output, the most widely used methods include logistic regression, decision trees, and neural networks. These three methods are thought to be multivariate function estimators. They all have their own advantages and disadvantages in solving the practical prediction or classification problem. The next section gives an overview of the methods [POT98, KLE94, BRE84, HOL97, ZAD90, HAY99, HAS95].

\subsubsection{Logistic regression}

Multiple linear regression is the most common form of predictive modeling. It posits a continuous response variable as a linear combination of a set of predictors (input variables) and error. Thus, for a response variable $\mathrm{Y}$ and input variables $\mathrm{X}$, the multiple linear regression equation has the form like:

$$
y_{i}=\alpha+\beta_{1} x_{1 i}+\beta_{2} x_{2 i}+\beta_{3} x_{3 i}+\ldots+\beta_{k} x_{k i}+e_{i}
$$

where $\beta_{\mathrm{k}}$ are the multiple regression coefficients, and $\mathrm{e}_{\mathrm{i}}$ is the error of prediction.

In a linear regression model, the training data is viewed geometrically. Then, each sample is a point in the predictive space. The regression equation describes the best fitted 'line' through these points. The disadvantage of linear regression is that it can be very sensitive to the data distribution.

The linear logistic regression model is a special case of generalized linear regression models. Since multiple linear regression is not appropriate for categorical 
output variables, the linear logistic regression model is a more proper representation for a situation when the output is categorical, especially dichotomous.

Logistic regression is a variation of ordinary multiple linear regression, which mostly deals with an application with binary response variable (usually coded 0 for control, and 1 for event). It produces a formula that predicts the probability (p) of the occurrence of an event as a function of the input variables. Actually, it fits a special Sigmoid shaped curve to the data by taking the above linear regression [KLE94]. The logistic regression equation is defined as:

$$
\begin{aligned}
& \operatorname{logit}\left(\mathrm{p}_{\mathrm{i}}\right)=\alpha+\beta_{1} x_{1 i}+\beta_{2} x_{2 i}+\ldots .+\beta_{k} x_{k i}+e_{i} \\
& \text { probability } \\
& \text { where } \quad \operatorname{logit}\left(\mathrm{p}_{\mathrm{i}}\right)=\ln \frac{p_{i}}{1-p_{i}} \quad \text { and }
\end{aligned}
$$

\begin{tabular}{|c|c|}
\hline 1 & $\mathrm{p}$ \\
\hline 0 & $1-\mathrm{p}$ \\
\hline
\end{tabular}

Taking the logarithm of the odds $\left(\frac{p_{i}}{1-p_{i}}\right)$ makes the repetition simpler. A simple exponential transformation converts the logarithm of odds back to probability:

$$
p=\frac{e^{\alpha+\sum_{i=1}^{k} \beta_{i} x_{i}}}{1+e^{\alpha+\sum_{i=1}^{k} \beta_{i} x_{i}}}
$$

There are two major stages for logistic regression analysis. The first step is to determine the above coefficients $\left(\alpha\right.$, and $\left.\beta_{\mathrm{i}}\right)$ in the model. The second step is to evaluate how well the regression model fits the observed data, which can be assessed by evaluating the prediction results on the validation dataset. The coefficients of the model are determined by maximizing the log form of the maximum likelihood function:

$$
L(p)=p^{\sum y_{i}(1-p)^{n-\sum y_{i}} \quad \text { and }}
$$




$$
\ln (L)=\sum y_{i} \ln p_{i}+\left(n-\sum y_{i}\right) \ln \left(1-p_{i}\right)
$$

The advantage of the logistic regression model for the classification application is that it is easy to construct the model, and interpret the results. Furthermore, logistic regression is popular partly due to its less restrictive assumptions. It does not assume linearity of relationship between the input variables and the response variable. The way of handling nonlinear effects is to use the nonlinear logit link function. Logistic regression also does not require the input variables and the error term to be normally distributed, and in general has less stringent requirements.

Because of the simplicity of the logistic regression classifier, it had been used in many classification and prediction applications. Malloy et al. used a multiple logistic regression model to study the relationship between maternal age, parity, marital status, and education with the age and cause of the infant death. Their results showed that smoking was associated with both neonatal and post-neonatal mortality [MAL88]. Logistic regression is also used to study the differences of sudden infant death syndrome and explained sudden infant death [LEA99].

For our study, the maximum likelihood multivariate logistic regression model, the stepwise regression method, which is provided by SAS Enterprise Miner, is used.

\subsubsection{Decision tree}

One of the most important family of classifiers are decision trees. There are two main types of decisions trees. One of them is classification tree, when the response variable is categorical. The other type is regression tree, when the response is continuous 
numeric values. Figure 6.1 shows the structure of a binary classification tree, using our data as an example.

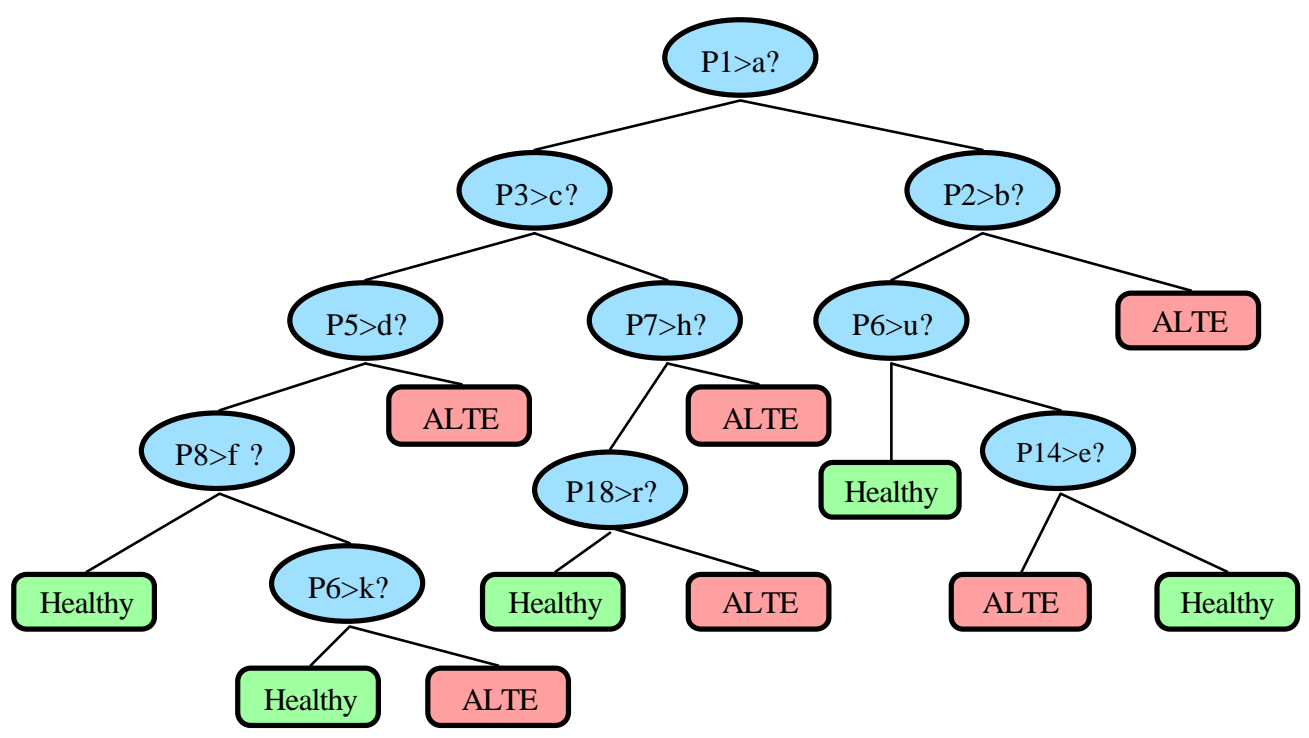

Figure 6.1 Structure of binary classification tree

A binary classification tree is built through a process called recursive partitioning, that iteratively splits the pattern space $\mathrm{R}^{\mathrm{d}}$ into subgroups according to a specific value (say a) of a parameter (say P1). Each sample will go either left, when P1>a, or right, when $\mathrm{P} 1<\mathrm{a}$. The partitioning will continue until the terminate nodes (leaves) are reached. The value of the cut-off threshold a for the parameter P1 must be optimized. The splitting criteria is based on minimization of the impurity function, which measures the impurity of the split subgroup [BRE84]. The most popularly used impurity functions include: (1) the probability of misclassification $\phi(p, 1-p)=\min (p, 1-p) ;(2)$ the Gini function $\phi(p)=$ $2 p(1-p)$; and (3) the binary entropy function $\phi(p)=-p \log p-(1-p) \log (1-p)$ [KUL98].

Based on the splitting criteria, a classification tree can be grown according to the specific training dataset. Complexity of the classification tree is determined by the number of leaves of the tree, which is also called the depth of the tree. The leaves are the 
terminate nodes of the tree. More leaves enable the tree to fit the training data perfectly, but might cause decreased generalization. The right depth of the tree should be chosen when the error rate starts to increase for the validation dataset. There are two ways to determine the right-size tree, pre-pruning and post-pruning. Pre-pruning uses forward stopping rules to stop the growth of a tree. The common stopping rule is to stop when the split is not statistically significantly minimizing the impurity function. For the postpruning method, growing a large tree first, and then pruning back to find the best 'subtree' based on its performance on the holdout validation data. The pre-pruning is less computational demanding, but it runs the risk of missing a good split that is after a weak split [POT98]. Thus, the common strategy of growing a right size tree is to grow a large tree that over fits the training data and then use a post pruning to improve the generalization performance [HOL97].

There are many algorithms for growing a decision tree. The most commercially popular algorithms are classification and regression trees (CART), Chi-squared automatic interaction detection (CHAID), and C4.5. For CART, the Gini index was used as splitting criterion and post-pruning strategy was chosen. While the CHAID used pre-pruning methods and the probability of misclassification as splitting and stopping criterion. C4.5 is a decision tree algorithm developed by Ross Quinlan, and played an important role in machine learning. Its splitting criterion is based on binary entropy function. Post-pruning was done to get the right size tree.

The advantages of the decision tree are apparent. The classifier is transparent and easy to interpret. The structure of the tree is very useful to assess which variables are important in the classification, and how they interact with each other. Furthermore, the 
trees are usually invariant under monotone transformation of the data. Once the tree is built, it can be easily used for classifying a new sample. The main drawback of the trees is its instability. The structure of the tree might be sensitive to the perturbations in the training dataset.

Decision tree classifiers have been used in the research of SIDS [VAL88], because both decision trees and neural networks are good for investigating complex medical problems with large sets of clinical and physiological data. Several HRV parameters, such as coefficient of variation, coefficient of variation of $\Delta R R$, RSA, respiratory rate and average values of $\mathrm{HR}$ were used as input to a rule-based expert system generator. The results showed that even though the trend of increased HR and decreased HRV in the SIDS infants was statistically non-significant, an expert system, which is based only on cardiorespiratory data, might be helpful in defining decision rules to identify SIDS cases.

The tree model provided by SAS Enterprise Miner is used for our infant classification problem.

\subsubsection{Artificial neural network}

Investigation and imitation of the learning and decision-making procedure of the human brain has been a hot topic for decades. One of the first brain models was provided by McCulloch and Pitts in 1943. They described the single neuron as the basic computational unit, which can compute any arithmetic or logical function [ZAD00]. Artificial neural network is a system modeled on the human brain. It attempts to simulate the multiple layers of the biological neurons by hardware or software. 
Understanding how real neural systems achieve their remarkable computational abilities is the first step to mimic their function. A biological neural system has ten billion highly interconnected neurons acting in parallel. Each neuron can connect with as many as 200,000 other neurons through synapses. All biological neurons have four basic components that fulfill four functions: the dendrites are used to accept input signals, the soma is used to process the input signals, the axon is used to transfer signals from input to output, and synapses are used to connect with other neurons. The power of the brain comes from the numbers of these basic computational units and the multiple connections between them. The brain is thought to be a highly complex, nonlinear and parallel computer. Nonlinearity is the most important property.

The basic unit of artificial neural networks is the artificial neurons, which simulates the four basic functions of the biological neurons. However, it is much simpler than the real neuron. The block diagram in figure 6.2 shows the model of an artificial neuron.

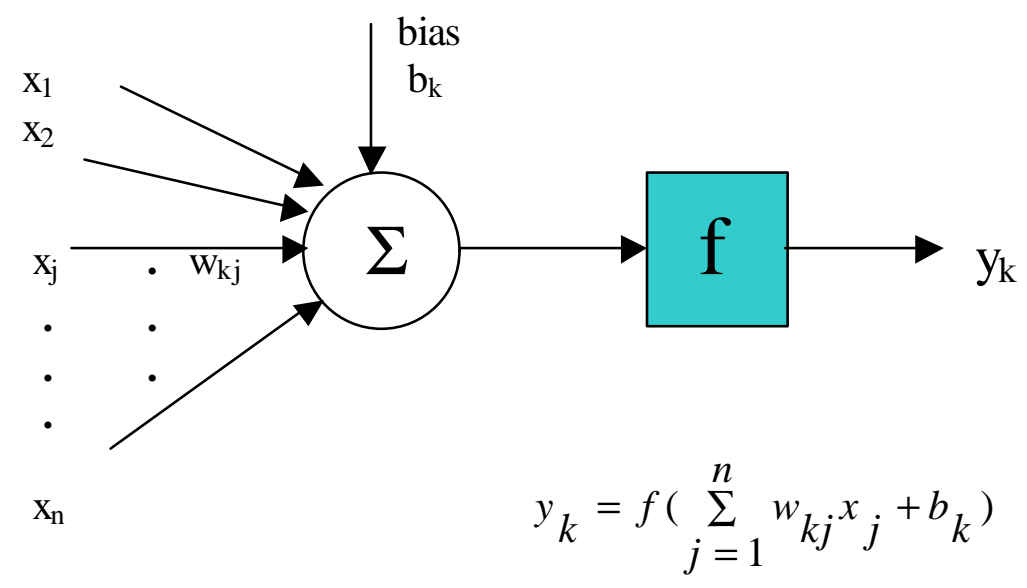

Figure 6.2 Block diagram of an artificial neuron 
In the equation for a neuron, $\mathrm{x}_{1}, \mathrm{x}_{2}, \ldots, \mathrm{x}_{\mathrm{n}}$ are the input signals; $\mathrm{w}_{\mathrm{k} 1}, \mathrm{w}_{\mathrm{kj}}, \ldots, \mathrm{w}_{\mathrm{kn}}$ are the synaptic weights of this neuron $\mathrm{k}$; $\mathrm{b}_{\mathrm{k}}$ is the bias; $\mathrm{f}$ is the activation function; and $\mathrm{k}_{\mathrm{k}}$ is the output of this neuron. The weighted inputs are summed and a bias is added. The result is passed through a nonlinear function (f), or activation function, to generate the result of this neuron. A Sigmoid function is the most common form of activation function to construct the neural network, due to its balance between linear and nonlinear behavior [HAV99]. The nonlinear activation function introduces nonlinearity to the network.

The artificial neural network is a parallel, distributed information processing structure consisting of these basic artificial neurons. These neurons are grouped into layers. Basically, all artificial neural networks have a similar structure of topology. Figure 6.3 shows the structure of a popular multilayer feedforward neural network.

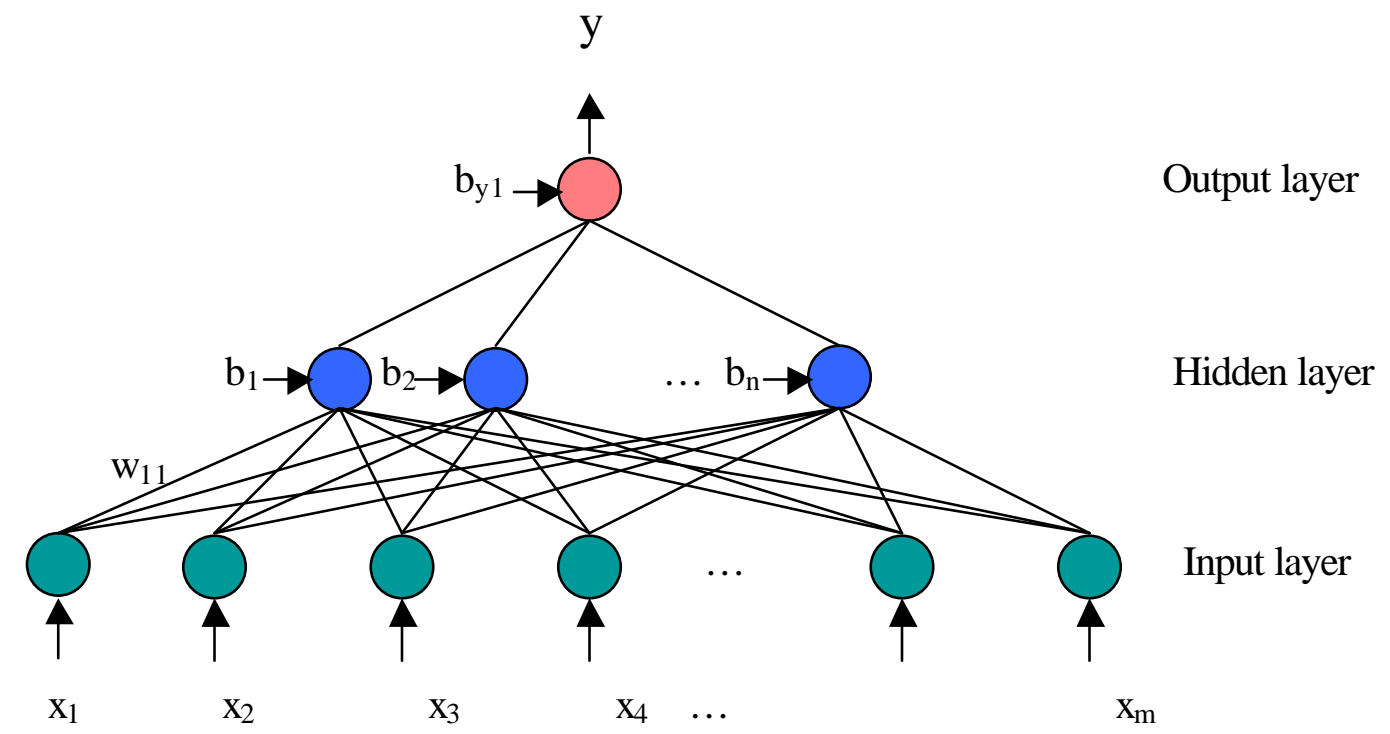

Figure 6.3 Multilayer feedforward neural network

The neurons in the input layer receive external input signals, weight them and pass them to the neurons of the hidden layer. The number of input neurons is dependent on the number of input variables. Neurons in the output layer receive a linear 
combination of the outputs from the hidden neurons and transform it into a suitable expected value of the response by the output activation functions. The output activation function depends only on the scale of the response variable. The hidden layer connects the external input and the network output. Each hidden neuron outputs a nonlinear transformation of a linear sum of its inputs. Having one or more hidden layers enables the network to extract higher-order statistics. It is the hidden neurons that add flexibility into the neural network, and make it more powerful than traditional linear regression methods for classification and prediction. The neural network is made to learn from the training samples, and is generalized for the new dataset. In general, the performance of a neural network is determined by the structure of the network, the learning algorithm, and the activation function of its neurons.

Artificial neural networks (ANN) are powerful non-parametric classifiers. They are a class of flexible nonlinear models used for supervised prediction problems. In many applications, it is preferred over the well-developed statistical methods. Compared with other prediction models, the main advantages of ANN classifiers include non-parametric adaptive learning, high nonlinearity, robustness, and plausibility for hardware implementation. There are also drawbacks of the ANN classifiers, such as its incomprehensibility. However, an effective classifier is much better than a more understandable but less effective model.

Neural networks have been used extensively for many practical classification and prediction applications, such as signal processing, control, and pattern recognition. They are powerful tools in medical data analysis, because they are good for datasets having nonlinear relationships and because they require a large training dataset, which is often 
satisfied in medical data analysis. There is much literature about using neural network classifiers to predict events in the medical area, such as predicting the risk of coronary heart disease [AZU97], trauma deaths [DIR00], and breast cancer survival [BUR94]. For instance, Azuaje, F. et al. used a neural network to predict the risk of coronary heart disease based on short-term heart rate variability [AZU97]. The input variables they used are Poincare-plot-encoded heart rate variability patterns. The output is a classification of risk: high, medium, or low risk. A three-layer perceptron with standard back-propagation algorithm was used. Their results showed that ANN is a successful approach to detect patients at different risks of coronary heart disease. Furthermore, they expected better results by using more input variables and improving the preprocessing techniques. DiRusso, S.M. et al. concluded that an ANN model can predict trauma death with good prediction accuracy compare with other trauma severity scoring systems [DIR00]. There are also applications of ANN in the study of sudden infant death syndrome, lifethreatening events and infant apnea. It has been used in the previous studies to recognize apnea [ANA93] and to classify respiration patterns in order to predict potential victims of SIDS [WIK95]. However, few studies have used ANN to help the prevention of SIDS or life-threatening events in infants.

The learning ability of the neural network is determined by its structure and by learning algorithms. The design of the structure of the neural network includes choosing a proper number of hidden layer and hidden neurons. More hidden layers make the network more flexible, complicated and powerful. However, it will cause the problem of higher computation expenses and over fitting, which will then lead to bad generalization on test datasets. It is widely accepted and proven that a multilayer perceptron with one hidden 
layer is a universal approximator, it can theoretically approximate any continuous surface to any degree of accuracy [POT98]. Furthermore, many studies also confirmed this. This kind of shallow network with appropriate learning algorithm works for almost all classification and prediction applications.

Another aspect of network design is to choose the learning algorithm. There are many supervised learning methods which can be chosen for the popular feedforward network. Among them, back-propagation (BP) algorithm is the most well-known and widely used leaning method, and it has been proven to be a highly successful algorithm in training a multilayer feedforward neural network for classification and prediction applications. Compared with traditional methods, BP is much better at constructing rules based on the training data. It enables the network to recognize patterns that similar to what have been learned. Here is a summary of the standard BP algorithm. It is an iterative gradient computing method designed to minimize the mean square error between the output of the network and the desired output.

Back-Propagation Learning Procedure:

1. Initialize weights $\left(\mathrm{w}_{\mathrm{ij}}\right)$ and bias with random values.

2. Give the network input variables and the desired output. For neural network classifier, the desired output is usually set to 0 (no event) and 1 (event).

3. Calculate the output of the network.

4. Adjust the weights using a recursive algorithm, starting from the output layer back to the first hidden layer:

$$
w_{i j}(t+1)=w_{i j}(t)+\eta \cdot \delta_{j} \cdot x_{i}^{\prime}(t)
$$


where $w_{i j}(t)$ is the weight from neuron $i$ to the neuron $j$ at time $t, x^{\prime}{ }_{i}(t)$ is the output of neuron $\mathrm{i}$ at time $\mathrm{t}, \eta$ is the learning rate, which determines the amount of correction on weight, and $\delta_{\mathrm{j}}$ is the error from neuron $\mathrm{j}$.

The error term for an output neuron is defined as:

$$
\boldsymbol{\delta}_{j}=y_{j}\left(1-y_{j}\right)\left(d_{j}-y_{j}\right)
$$

Where $y_{j}$ is the actual output the neuron, and $d_{j}$ is the desired output.

The error term for a hidden neuron is:

$$
\boldsymbol{\delta}_{j}=y_{j}\left(1-y_{j}\right) \sum_{k} w_{j k}(t) \cdot \boldsymbol{\delta}_{k}
$$

Where $y_{j}$ is the actual output of the hidden neuron, $w_{i k}(t)$ is the weight between this neuron and the neurons of next layer, and $\delta_{\mathrm{k}}$ is the error of the associated neuron in next layer.

5. Repeat steps 2 to 4 until a satisfactory training precision reached.

The determination of the learning parameter, such as learning rate, and learning precision, will also have an effect on the learning procedure. The learning rate somewhat determines the learning time. Typically, it is chosen to be less than or equal to $1 / \mathrm{N}_{\text {inputs. }}$ The learning precision also affects the performance of the neural networks; higher precision will result in good learning but worse generalization. Usually, the learning precision is $2-5 \%$. Choosing an appropriate activation function for the hidden and output neurons is another consideration. The most widely used activation function is a sigmoid function. It is better to start with a sigmoid function, and modify it, if necessary. The standard BP algorithm has been proven to do very well in most applications. There is still some means of improving its performance. There are two modified BP Algorithms, (1) 
using a momentum term $\alpha$ to speed up the convergence of the algorithm without adding instability into the system. The weight adjusting formula is changed to:

$$
w_{i j}(t+1)=w_{i j}(t)+\boldsymbol{\eta} \cdot \boldsymbol{\delta}_{j} \cdot x_{i}^{\prime}(t)+\boldsymbol{\alpha}\left[w_{i j}(t)-w_{i j}(t-1)\right]
$$

(2) Setting a gradually increasing learning precision instead of a fixed one. It has been shown that the second modified BP algorithm almost doubles the training speed, compared other two algorithms [KOT91].

We expect satisfactory result for our study from neural network. However, the capability of the neural network is limited by the quality of the training data. Some preprocessing methods are needed to extract proper features for the neural network. This is also true for other models.

\subsubsection{Comparison of the three models}

As described above, the three most widely used supervised prediction models are generalized linear regression, decision trees, and neural networks. All three models are thought to be multivariate function estimators. For a binary response variable, all of them can be used to estimate the probability of the events. Each model has its own advantages and disadvantages, such as the regression model is much smoother than others, while the neural network is highly flexible, but easy to over fitting. Figure 6.4 compares them visually [POT98]. 


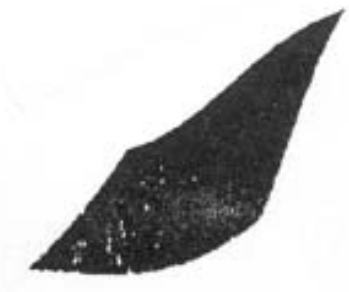

Generalized Linear Models

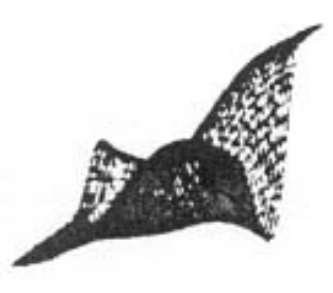

Neural

Networks

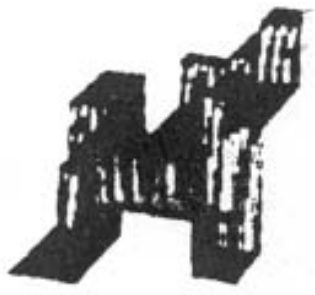

Decision

Trees

Figure 6.4 Comparison of the three models

Compared with traditional statistical and artificial intelligence methods, neural networks are a new approach for information processing. It is an intrinsically nonparametric and model-free method, which doesn't not require rule development. In many applications, such as medical data analysis, rules are not always easy to develop. There are also similarities between neural networks and the statistical regression models. Logistic regression model can be represented as feedforward neural networks with no hidden layers and an identity output activation function. The simple structure makes it easier to interpret and less troublesome to train. On the other hand, neural networks have been categorized as a form of nonlinear regression. Thus, neural networks can do everything that linear and logistic regression can do, and more. The drawback of a neural network is that it is a black box, thus it is impossible to interpret the classification. However, in many applications, comprehensibility is not always a crucial feature of a prediction model. A model with high prediction accuracy might be preferred over other more understandable but less effective models.

The most important factor in choosing the classification model is stability, which is another way to represent the generalization performance. Both decision trees and 
neural networks face the problem of over fitting, where it learns too much from the training data, which limits the capacity for generalization for the testing data. Decreasing the complexity of the model, such as using fewer hidden neurons and fewer leaves of the tree, is one way to solve the over fitting problem.

From the theoretical description of the three models, the advantages and disadvantages of these three models are apparent. However, it is hard to say which is better than others for a specific application. A good way of choosing a better model is to compare the generalization performance of the models for the specific classification problem. There are also studies done to compare these three predictive models in medical diagnosis applications. Burke, et al. compared many statistical predictive models with different types of neural networks for predicting cancer survival. The statistical models they included were principal components analysis, classification and regression trees, and logistic regression. They found that neural networks improved the predictive accuracy significantly, and concluded that neural network can perform as well as the best traditional prediction methods [BER942]. Selker et al. compared the relative performance of the three models (logistic regression, decision tree, and neural network) in predicting acute cardiac ischemia, which includes acute myocardial infarction and unstable angina pectoris. The area under receiver-operating characteristic (ROC) curve was used to compare the predictive accuracy. Their results showed that neural networks had the best prediction accuracy, no matter whether they used a limited or unlimited number of input variables. However, they concluded that it is still impossible to prove which model is intrinsically more powerful. Choice between these methods should be determined for a specific application dependent problem [SEL95]. 


\subsection{Materials and Methods}

Both traditional statistical analysis methods and pattern recognition techniques are used to investigate the possibility of using heart rate variability to differentiate and classify infants with regarding to future life-threatening events (FALTE). Logistic regression, decision tree, and neural network models are used to classify the infants.

For the infant classification study, input variables may include most of the HRV parameters calculated from the 8-hour PSG study as described in Chapter 3, and two more related parameters: REM duration, and the ratio of REM time over all sleep state time. Our goal is to use statistical or pattern recognition methods to predict future events for a specific infant.

\subsubsection{Event classification}

In the CHIME study, infants were monitored at home following the PSG for up to several months. The home monitor recorded all physiological signals for 75 seconds before and 30 seconds after an apneaic or bradycardic event. Each event was overread by an expert and classified [RAM01]. The infants in the CHIME dataset are then classified into two mutually exclusive groups, healthy infants (control) and infants with serious future life-threatening events (event). In this section, we discuss the method used to categorize the event infants.

There are two types of life-threatening events studied by CHIME, apnea and bradycardia. Events are categorized as extreme events and conventional events, according to whether they exceed the conventional or extreme thresholds set by CHIME researchers. The thresholds set for extreme events are: (1) apnea for at least 30 seconds; 
(2) heart rate less than $60 \mathrm{bpm}$ for at least 10 seconds of an infant at less than 44 weeks' PCA; or (3) heart rate less than 50bpm for at least 10 seconds of an infant who is at least 44 weeks of PCA. The conventional thresholds are defined as follows: (1) apnea for at least 20 seconds; (2) for infant with less than 44 weeks' PCA, heart rate ess than $60 \mathrm{bpm}$ for at least 5 seconds or less than $80 \mathrm{bpm}$ for at least 15 seconds; or (3) for infant with at least 44 weeks' PCA, heart rate less than $50 \mathrm{bpm}$ for at least 5 seconds or less than $60 \mathrm{bpm}$ for at least 15 seconds [RAM01]. All events were overread by an expert and categorized. During 718358 hours of home monitoring, 11000 events exceeded the conventional alarm threshold. Of these, 931 were extreme events.

Only infants who had a PSG were considered for this study. According to these recorded events, infants are classified into two mutually exclusive groups, healthy infants (control) and infants with serious future life-threatening events (FALTE). An infant is classified as FALTE if it has at least one extreme event or five or more conventional events after the PSG. Such infant classification information is necessary for our supervised classification study. Furthermore, our goal is to predict whether the infant have future life-threatening events, using the $\mathrm{RR}$ interval and derived heart rate variability from the PSG. Therefore, only the events recorded after the PSG are taken into account for this dissertation.

The total hours of monitor use is another issue to be considered when classifying infants. The hours of monitor usage for infants vary from less than one hour to 10880 hours. Insufficient monitor use will not provide enough information to classify infants. We only include infants who used the home monitor for at least 200 hours. Satisfying all 
the restrictions, 122 infants are included in the further statistical analysis and classification study. 20 of them are in the event group.

\subsubsection{Training and testing datasets}

The entire dataset, which contains data for 122 infants, is divided randomly into two parts as a training dataset and test dataset. $70 \%$ of the dataset (85) is used to fit the model (or train the neural network), which is called the supervised learning process. The other $30 \%$ (37) is used as testing data. The training dataset is also used to choose the best model from many selections, such as statistical regression model, decision tree, or neural network of different structure. Different thresholds are tested on the test dataset to achieve the best generalization performance. Lastly, a further holdout dataset, test dataset, is needed for a final, unbiased assessment of the generalization of the chosen model. However, not enough data is available for a holdout test set in our study.

The response variable is group and the final decision is which group does the specific infant belong to (denoted 1 for the future events and 0 control). The initial set of major predictor variables are the HRV parameters of each infant. Other input variables include sleep state code (00: QS, 01: REM, and 10: AWK), because HRV varies due to different sleep states, REM duration, and ratio of REM sleep to all sleep wake states, because a study done by [BAK77] determined that reduced REM sleep might be associated with a high risk of sudden infant death. The classification models are highly dependent on the training dataset. It is very important to choose appropriate input variables for the predictive models. Regression will have problem when there are highly 
correlated input variables, which is called collinearity. For a neural network, too many inputs can prevent the network from converging on a good model during training.

Our study on the relationship of the HRV parameters helps to determine the input variables for our infant classification problem. For the first part of the infant classification study (section 6.3), we chose one of the HRV parameters from the known highly correlated pairs, and the input variables include Mean, SDNN, RMSSD, HFW, LFW, LFW/HFW, ApEn, Poincare, fractal dimension, REM duration, REM/total sleep-wake, and sleep state code. Then, a reduced input set was selected for section 6.4-6.5, and include: Mean, SDNN, RMSSD, ApEn, Poincare, REM duration, REM/total sleep-wake, SDANN, SDNNIDX, PCA, and sleep state code.

Since HRV measurements are calculated for all 5-min segments of the PSG for each infant, there is a vector of 5-min HRV parameters for each sleep state per infant. We use the results of these 5-min segments and treat each of them as one independent observation. After fitting the model with the learning dataset, we also use all the 5-min segments data of an infant for the prediction. Therefore, we will get a vector of group decisions for each infant, because each infant has a number of 5-min segments in QS/REM/AWK. A rule is needed to make a final decision about which group the infant belongs. We investigate different rules, such as: if $50 \%$ percent of the 5-min segments from the PSG predict an event, this infant will be classified into the event group. A relative operating characteristic (ROC) curve is used to choose the best model according to varying thresholds. 


\subsection{Classification of Infants using SAS Enterprise Miner}

SAS Enterprise Miner is a powerful software tool that provides an end-to-end solution for data mining. Data mining has been defined as the nontrivial extraction of implicit, previously unknown, and potentially useful information from large amounts of data [FRA92]. It allows using the collected data to its fullest. In data mining, machine learning, statistical and visualization techniques are used to discover and present knowledge from the existing data. Its ultimate goal is to reach a good prediction model.

Enterprise Miner provides a full range of integrated models and algorithms, including regression, decision trees, neural networks, memory based reasoning, clustering, etc. It is a very convenient tool for both supervised and unsupervised classification. The most popular supervised classification models, logistic regression, decision tree, and neural network, of the Enterprise Miner are chosen here for our infant classification task.

For each model, several parameters will be used to evaluate the model: sensitivity, specificity, and misclassification rate. These values will be calculated for both the training and testing datasets. The models output a number, typically between 0 and 1 , which can be used to make a classification of event or non-event for each 5-minute segment. For sections 6.3.2, 6.3.3, and 6.3.4, a set threshold of 0.48 is chosen to make the distinction and the results are calculated based on this threshold. These are the results of all 5-minute segments from all infants with no distinction made for infant. In section 6.3.5, a ROC will be used to further explore this threshold. In 6.3.6, we will explore an algorithm for classifying an infant based on the results for all 5-minute segments from that infant. A ROC plot will also be used to evaluate the models. 


\subsubsection{Insight into the input data}

As a powerful statistical analysis tools, SAS Enterprise Miner makes it very easy to analyze the statistical characteristics of the input and output variables, including the ability to view the distribution of each variable. Table 6.1 gives the statistical summary of the input variables for the infant dataset. Figure 6.5 show the distribution of some HRV parameters obtained from all 5-min segments of the 122 infants. Besides these input variables in the table, there is also a categorical input variable, sleep state code. Group information of the infants is set as the target variable. All of the 5-min segments' of data from the randomly chosen training and testing infants are treated separately as training and testing samples, respectively.

Table 6.1 Statistical summaries of the input interval variables

\begin{tabular}{|c|c|c|c|c|c|c|c|}
\hline Name & Min & Max & Mean & Std Dev. & Missing 7 & Skewness & Kurtosis \\
\hline MEAN & 265.04 & 614.73 & 418.4 & 59.357 & $0 \%$ & 0.3602 & -0.003 \\
\hline MED IAN_ & 218 & 613 & 416.79 & 60.415 & 07 & 0.2856 & -0.032 \\
\hline SDNN_ & 3.3679 & 151.47 & 26.407 & 13.586 & $0 \%$ & 1.4561 & 5.8359 \\
\hline IQRNN & 4 & 188.13 & 33.02 & 20.391 & $0 \%$ & 1.8057 & 5.4259 \\
\hline NIQRNN & 0.0105 & 0.863 & 0.0805 & 0.053 & 07 & 3.0663 & 26.766 \\
\hline $\mathrm{CU}$ & 0.884 & 44.386 & 6.3858 & 3.3967 & $0 \%$ & 2.0944 & 13.661 \\
\hline SDSD_ & 1.0292 & 32.24 & 8.0245 & 4.2606 & $0 \%$ & 1.2877 & 3.0097 \\
\hline IQRSD & 1 & 38 & 8.3999 & 5.1156 & $0 \%$ & 1.4261 & 3.1793 \\
\hline NIQRSD & 0.0026 & 0.0917 & 0.0196 & 0.0106 & $0 \%$ & 1.1859 & 2.051 \\
\hline RMSSD & 1.6313 & 54.417 & 11.591 & 6.3346 & $0 \%$ & 1.6173 & 5.3054 \\
\hline cus & 0.435 & 8.9689 & 2.7176 & 1.2719 & $0 \%$ & 0.9723 & 1.5568 \\
\hline PNN50 & $1 E-7$ & 0.3347 & 0.0087 & 0.0242 & $0 \%$ & 7.3946 & 75.824 \\
\hline HF & 220.6 & 471708 & 23416 & 33173 & $0 \%$ & 4.8747 & 38.958 \\
\hline LF & 751.71 & $5.89 \mathrm{E} 6$ & 126663 & 224203 & 07 & 11.685 & 245.26 \\
\hline NHF & 1.1913 & 95.508 & 21.373 & 17.926 & 07 & 1.4612 & 1.5902 \\
\hline NLF & 4.4923 & 98.809 & 78.627 & 17.926 & $0 \%$ & -1.461 & 1.5902 \\
\hline TOTAL_POWER & 986.56 & $6.15 E 6$ & 150079 & 241458 & $0 \%$ & 10.751 & 215.48 \\
\hline LF_HF & 0.047 & 82.939 & 8.1359 & 8.7383 & $0 \%$ & 2.9965 & 13.781 \\
\hline HFW & 1491.8 & $2.49 \mathrm{E} 6$ & 154493 & 200286 & $0 \%$ & 4.6349 & 35.071 \\
\hline LFW & 7797.4 & $1.23 \mathrm{E} 7$ & 681090 & 860371 & $0 \%$ & 4.744 & 42.038 \\
\hline LFW_HFW & 0.0732 & 46.995 & 6.1834 & 5.3739 & $0 \%$ & 2.3741 & 9.3798 \\
\hline APEN & 0.0575 & 1.6319 & 0.8792 & 0.2742 & 07 & 0.1858 & -0.403 \\
\hline POINCARE & 3.0768 & 130.43 & 24.868 & 15.338 & $0 \%$ & 1.8307 & 5.0917 \\
\hline FD & 1.03 & 1.5148 & 1.1692 & 0.0661 & $0 \%$ & 0.6607 & 0.5793 \\
\hline SDANN & 4.6908 & 87.62 & 23.922 & 11.882 & 07 & 1.1214 & 1.9006 \\
\hline SDNNIDX & 7.103 & 64.114 & 26.779 & 9.5438 & $0 \%$ & 0.3045 & 0.0526 \\
\hline REMT IME & 2940 & 18840 & 8924 & 3140.9 & $0 \%$ & 0.5181 & 0.158 \\
\hline REMRAT IO & 0.1125 & 0.6667 & 0.322 & 0.1138 & $0 \%$ & 0.571 & 0.1457 \\
\hline TIME & 1 & 52 & 13.701 & 9.6994 & 07 & 0.9214 & 0.5424 \\
\hline INFANT ID & 10012 & 55013 & 27667 & 14225 & $0 \%$ & 0.5647 & -1.015 \\
\hline
\end{tabular}


Percentage
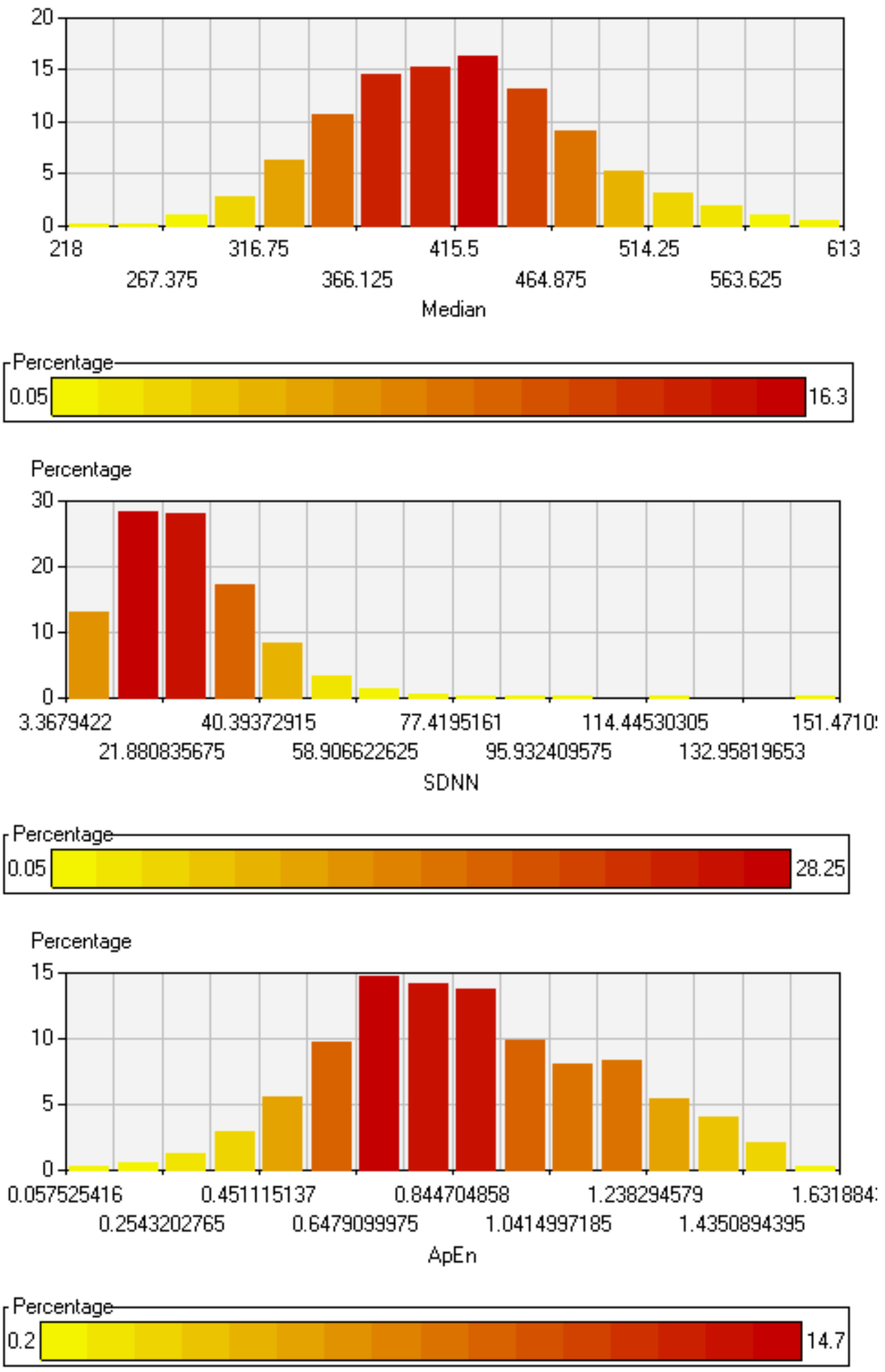

Figure 6.5 Distributions of Median, SDNN, and ApEn 


\subsubsection{Logistic regression}

Logistic regression model uses the following input variables: Mean, SDNN, RMSSD, HFW, LFW, LFW/HFW, ApEn, Poincare, FD, SDANN, SDNNIDX, REMtime, REMratio, and sleep state code (ssc10 and ssc20). Logit function is chosen as the link function for the logistic regression model, and the backward regression method is performed. The cross-validation misclassification rate for the training dataset is used as the criteria for choosing the best regression model. The cross-validation method is used when the dataset is too small to be split into separate training and validation datasets, and the same observation is used to both train and evaluate the model. For the training procedure, the process of cross validation in SAS Enterprise Miner approximates a process in which an observation is left out of the training data, the rest of the training dataset is used to train the model, and the hold out training sample is used to evaluate the model. This procedure is repeated for each training sample and the misclassification rate is computed for all these hold out evaluation samples as a criterion for model selection. For this study, this was performed only on the training set. The test set was still reserved as an independent dataset.

Table 6.2 shows the estimates of the final chosen logistic regression model. Therefore, the best logistic regression model for our infant classification is generated based on these estimates.

$$
\begin{aligned}
\log i t(p)=\ln \frac{p}{1-p}= & 16.6+31.3 \text { REMtime }-12.4 F D-2.28 H F W \\
& +0.64 \text { ssc } 10+0.22 \text { ssc } 20+0.27 \text { RMSSD } \\
& +0.02 \text { SDNNIDX }+0.03 \text { LFW_HFW }-0.02 \text { Mean } \\
& -0.001 \text { REMratio }-0.01 \text { SDNN }
\end{aligned}
$$


where $\mathrm{p}$ is the probability for having an event. The most important HRV parameters used in the logistic regression model are duration of REM sleep, fractal dimension, and high frequency power. The ANOVA table of this regression analysis is in appendix E.

Table 6.2 Estimates of the Logistic Regression model

\begin{tabular}{|c|c|c|c|}
\hline Effect Name & Effect Label & Parameter Estimate & Effect T-scores \\
\hline $\mathrm{ApEn}_{\mathrm{p}}$ & ApEn & & \\
\hline $\mathrm{FD}$ & $\mathrm{FD}$ & -12.42775577 & -6.134466306 \\
\hline HFW & HFW & $-2.278784 \mathrm{E}-6$ & -3.093168019 \\
\hline Intercept & Intercept & 16.604896906 & 6.6480631203 \\
\hline LFW & LFW & & \\
\hline LFW_HFW & LFW/HFW & 0.0274320832 & 3.2423484342 \\
\hline Mean & Mean & -0.022876624 & -13.50017245 \\
\hline Poincare & Poincare & & \\
\hline REMratio & REMratio & 31.294685589 & 14.569974733 \\
\hline REMtime & REMtime & -0.000938559 & -11.9700866 \\
\hline RMSSD & RMSSD & 0.2749487949 & 7.2369690724 \\
\hline SDANN & SDANN & & \\
\hline SDNNIDX & SDNNIDX & 0.0198979263 & 2.5276384789 \\
\hline SDNN_ & SDNN & -0.010015099 & -1.980574789 \\
\hline $\operatorname{ssc} 10$ & $\operatorname{ssc} 10$ & 0.636221822 & 6.5555258937 \\
\hline $\operatorname{ssc} 20$ & $\operatorname{ssc} 20$ & 0.2201691443 & 2.9792617871 \\
\hline
\end{tabular}

Sensitivity, specificity, and misclassification rate of the model in classifying all 5-min segments of training dataset are used to evaluate the model. Table 6.3 show the results of the logistic regression model for both the training and testing datasets. Fixed threshold on the output $(\mathrm{p}=0.48)$ is used to calculate sensitivity, specificity, and misclassification rate for all 5-minute segments. Sensitivity was quite poor for both the training and testing datasets.

Table 6.3 Results of the regression model in classifying 5-min segments for 0.48 threshold

\begin{tabular}{|c|c|c|}
\hline & Training dataset & Testing dataset \\
\hline Sensitivity & 0.1725 & 0.1675 \\
\hline Specificity & 0.9593 & 0.9964 \\
\hline Misclassification Rate & 0.1631 & 0.1422 \\
\hline
\end{tabular}




\subsubsection{Decision tree}

The same input variables as used in logistic regression are used to build the decision tree model. A binary tree is grown, according to a splitting criterion based on Chi-square test with a significant level setting at 0.02 . The maximum depth of the tree is limited to 12, and 100 observations are required for the splitting search. The post-pruning method is used. Compared with pre-pruning, post pruning is expensive in computation, but avoids the risk of missing a good split. Since the training data size of our study is not very large, we chose the more precise method. The proportion of correct classification of the training dataset is used as the model assessment measure. Figure 6.6 shows the structure of the tree which works best for our infant classification. Nineteen leaves (terminate nodes) are reached for the final structure of tree.

In terms of classifying all 5-min segments of the infants, the decision tree reached a very high sensitivity $(99.84 \%)$ and specificity $(94.41 \%)$ on the training data. The misclassification rate is $1 \%$. However, the sensitivity is not satisfactory for the testing dataset. Table 6.4 shows the results of the tree model in classifying all 5-min segments for both training and testing datasets. Different splitting criteria, including Chi-square, Gini index, and binary entropy function, as well as other parameters, such as maximum depth of tree, and observations required for a split search, were also tested. No significant differences were seen in the results.

Table 6.4 Results of the tree model in classifying 5-min segments for 0.48 threshold

\begin{tabular}{|c|c|c|}
\hline & Training dataset & Testing dataset \\
\hline Sensitivity & 0.9441 & 0.1340 \\
\hline Specificity & 0.9984 & 0.9545 \\
\hline Misclassification Rate & 0.0100 & 0.1827 \\
\hline
\end{tabular}




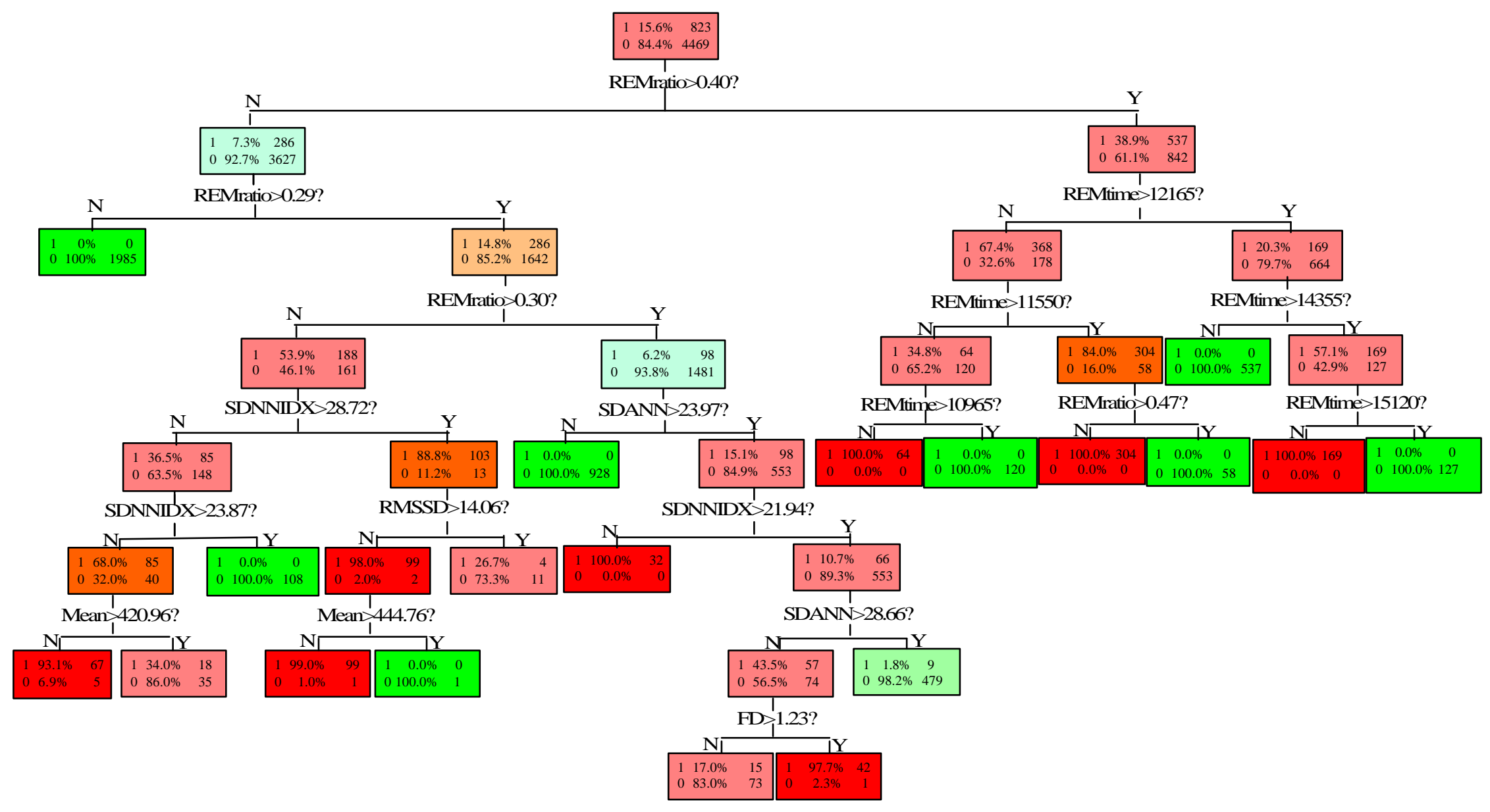

Notes: "1" indicates FALTE infants and "0" normal

Figure 6.6 Structure of the Decision Tree 


\subsubsection{Neural network}

The same inputs are used to test the neural network classifier. The popular multilayer perceptron neural network architecture is chosen for our infant classification task. Misclassification rate is used as the model selection criterion. Levenberg-Marquadt training technique is applied, which is described in detail in the next section.

Choosing an appropriate number of hidden neurons is critical to neural network models. Its selection is problem dependent, depending on the size of training data and the number of input nodes. The best method to solve this problem is to find the best neural network structure experimentally. Neural networks with different numbers of hidden neurons were tested and described in section 6.4. With an increasing number of hidden neurons, the misclassification rate for the training dataset is decreased. More hidden neurons enhance the flexibility of the network, but runs the risk of over fitting. Over fitting means the network learns too much detailed information from the training data, which reduces its generalization capability.

The best model is chosen based on the misclassification rate of the training datasets. However, the misclassification rate on the training data should not be very high. Very high learning preciseness might result in decreased performance on the testing dataset. We chose the neural network model with 8 hidden neurons. Table 6.5 gives the sensitivity, specificity and misclassification rate of the neural network model in classifying 5-min segments for both the training and testing datasets. Sensitivity is quite poor for both the training and testing datasets. In section 6.4 and 6.5 , we explore the neural network in greater detail and assess other aspects of the dataset to gain insight into these results. 
Table 6.5 Results of the neural network model in classifying 5-min segments for 0.48 threshold

\begin{tabular}{|c|c|c|}
\hline & Training dataset & Testing dataset \\
\hline Sensitivity & 0.3341 & 0.0773 \\
\hline Specificity & 0.9821 & 0.9829 \\
\hline Misclassification Rate & 0.1187 & 0.1685 \\
\hline
\end{tabular}

\subsubsection{Comparison of three models}

Regression, tree, and neural network classification models are compared in this section. Figure 6.7 shows the diagram in SAS Enterprise Miner to compare these three models and give a final assessment of the models.

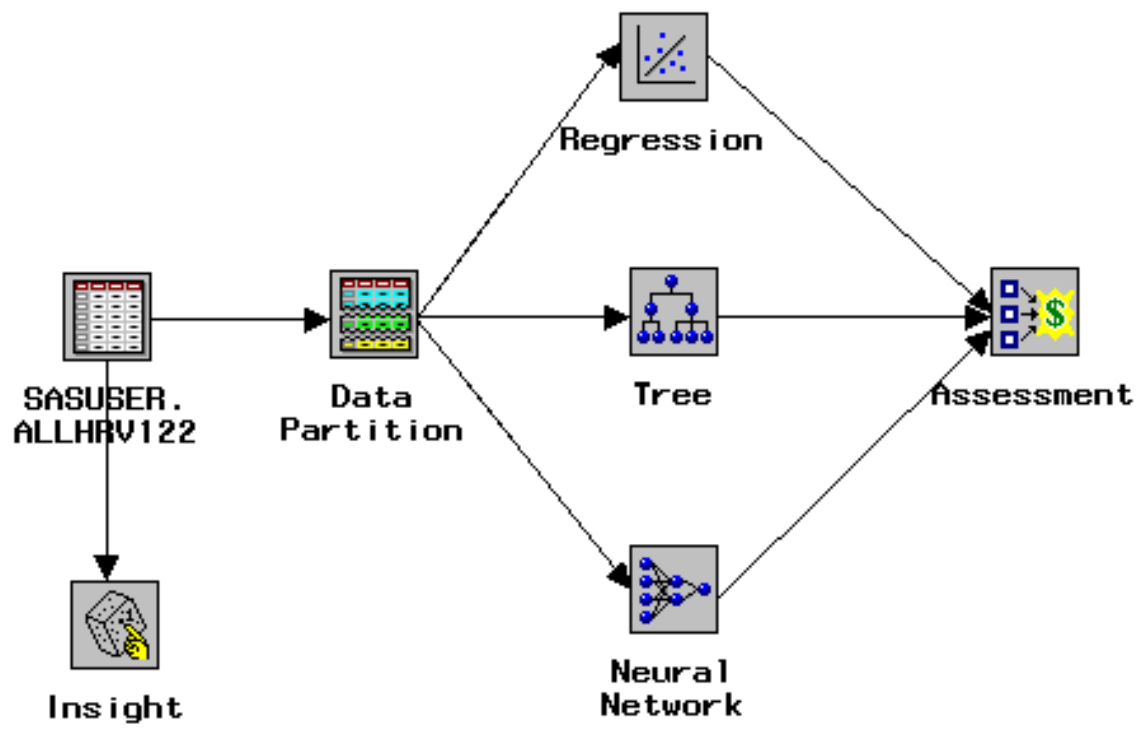

Figure 6.7 Diagram for the comparison of three classification models

All of the models reached a low misclassification rate in terms of classifying all of the 5-min segments of the infants, see table 6.6. An appropriate threshold must be chosen 
for the 5-min segments classification to calculate sensitivity and specificity. Different thresholds, ranging from 0.1 to 0.8 , are tested. Figure 6.8 uses the relative operating characteristic (ROC) curve to evaluate these three models in classifying all 5-min segments of the infants for the training and testing dataset. The decision tree achieves the best classification results for the training dataset, with $100 \%$ sensitivity and $87 \%$ specificity for 0.1 threshold. While the neural network and regression reached $82 \%$ and $66 \%$ sensitivity and $80 \%$ and $81 \%$ specificity for 0.3 and 0.2 , respectively.

Table 6.6 Misclassification rate in detecting 5-min segments of the classification models for 0.48 threshold

Misclassification rate

\begin{tabular}{|c|c|c|}
\hline & Training dataset & Testing dataset \\
\hline Logistic Regression & 0.1631 & 0.1422 \\
\hline Decision Tree & 0.01 & 0.1827 \\
\hline Neural Netwrok & 0.1187 & 0.1685 \\
\hline
\end{tabular}

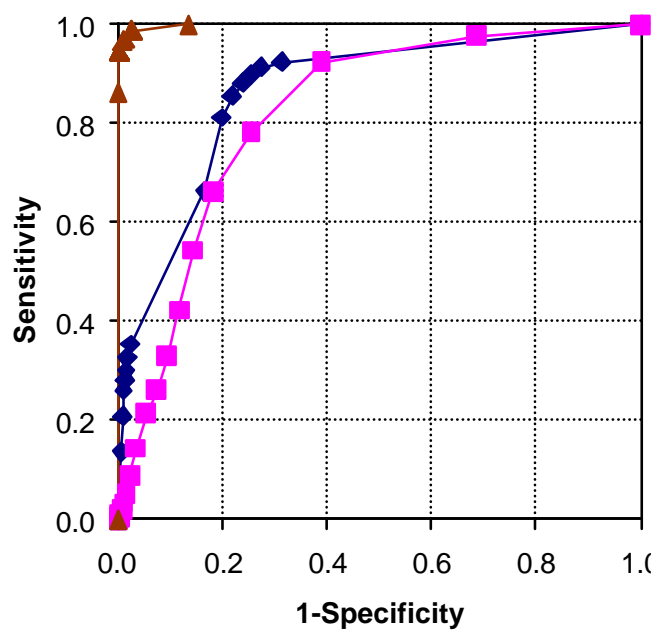

(a) Training dataset

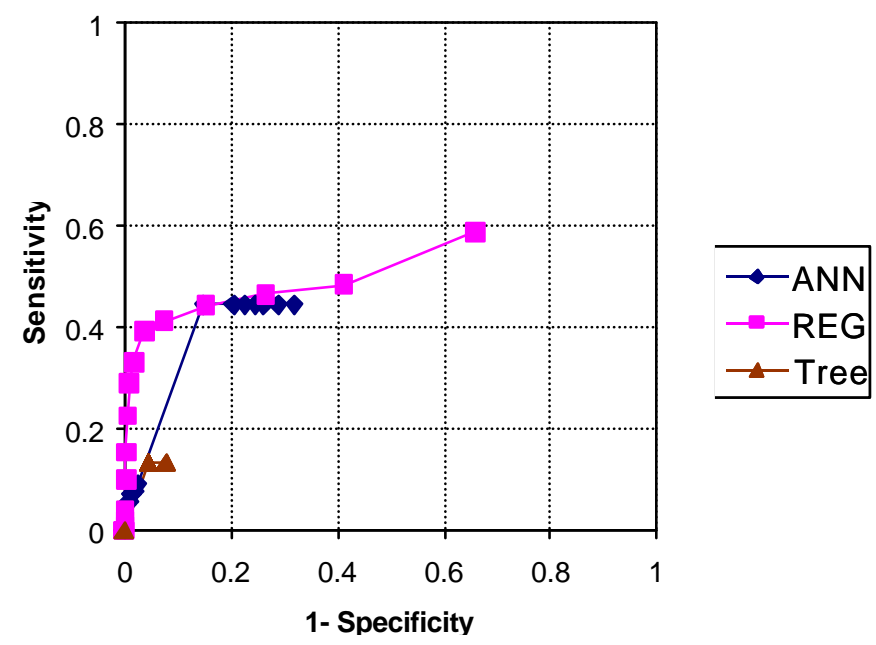

(b) Testing dataset

Figure 6.8 Relative operating characteristic curve of the models in classifying all 5-minute segments for training and testing sets 
In terms of classifying 5-min segments of the infant, the above results show that even though the misclassification rate is acceptable, the sensitivities of the models for the test set are very low. The highest testing sensitivity is 0.4845 . Neural network reached the best balance between sensitivity (0.4459) and specificity (0.8515). The thresholds, which reached the best balance between sensitivity and specificity, are selected as 0.25 and 0.3 for the training dataset and testing dataset, respectively.

For our specific infant classification problem, since the number of event infants is much less than the number of the healthy control infants, the number of 5-min segments is also less. During the training procedure, the misclassification criterion tends to lead the classifier to recognize more non-event 5-min segments in order to minimize the misclassification rate. This sacrifices sensitivity. Although many false alarms (low specificity) are not acceptable for infant classification, detecting event infants is critical. Improving the sensitivity of the classification model is a major task. We further explore the neural retwork model in section 6.4 to assess if structure has an impact on the results. In section 6.5, we consider other aspects of the data which may make classification difficult.

\subsubsection{Classification of infants}

Classifying all of the 5-min segments gives us a vector of prediction results for each infant. By setting a threshold, we are able to make a final decision for each infant, eg. if $10 \%$ of the 5 -min segment predictions resulted in an event, the infant will be classified as an event infant. Sensitivity is very important for our problem, because we do not want to miss many event infants. Lower thresholds increase the sensitivity. However, 
decreasing the number of false alarms is also important. A trade off must be made between sensitivity and specificity. Different thresholds are tested for all three models. Figure 6.9 are the relative operating characteristic curve of the models on the training and testing dataset.

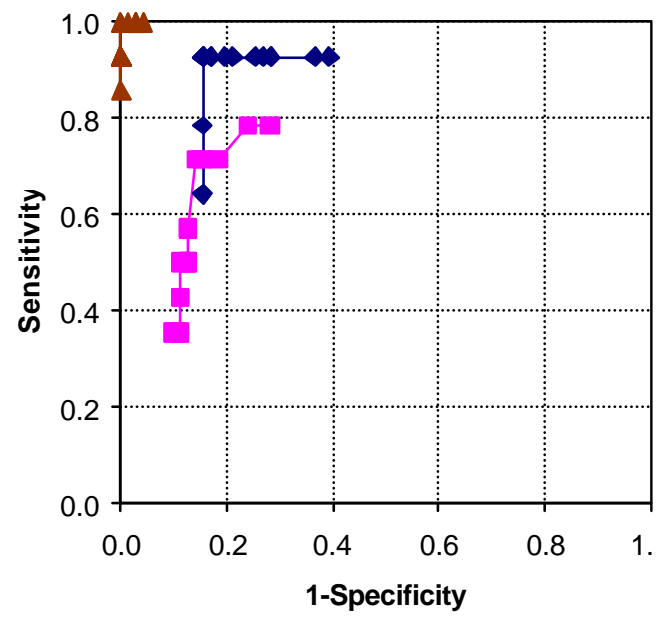

(a) ROC curve for training dataset

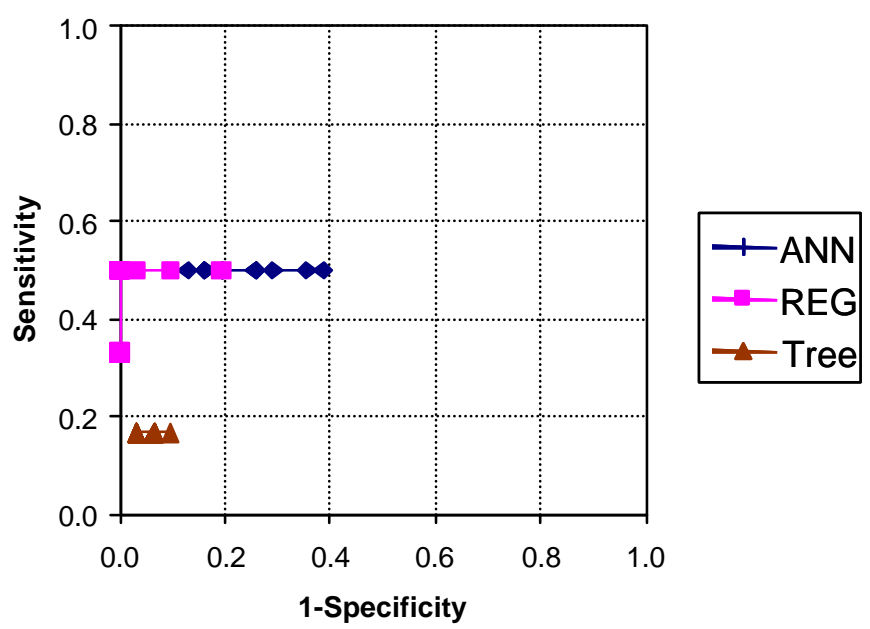

(b) ROC curve for testing dataset

Figure 6.9 Relative operating characteristic curve of three classification models for infant classification

The above ROC curves help to make a trade off between sensitivity and specificity, and to find the best prediction model. Although the training process is very successful, for the testing dataset, the highest sensitivity is $50 \%$ and $100 \%$ specificity for regression. The same sensitivity can be reached by neural network with a slightly lower specificity. The performance of the tree is disappointing: $97 \%$ specificity with less than $20 \%$ sensitivity. The above ROC curves also show that the decision tree has the best performance on the training dataset, which reached very high sensitivity as well as specificity. However its generalization to the test set is the worst. Logistic regression is 
the opposite. Neural network is in between. More exploration of the models is needed to achieve better generalization.

The sensitivity of the infant classification needs to be improved. However, if the best results (50\% sensitivity and $100 \%$ specificity) in the above study can be reached consistently for independent infant datasets, it still provides extremely valuable information for clinical use. Imagine the clinical application of this study, a PSG is taken for a newborn to predict whether he or she is going to have serious life-threatening events. There will be no false alarms (100\% specificity), and will capture and help half of the event infant population (50\% sensitivity). Since this application is for the whole newborn population, and the prediction of event will result in providing corresponding therapy or extra health care, a high false alarm rate is not affordable.

In the next section, neural networks are explored further to find out how network structures, and model performance function affect the classification results. Our goal is to improve the generalization characteristic of the classifier, reaching higher sensitivity without much sacrifice in specificity.

\subsection{Classification of Infant Group Using a Neural Network Toolbox in Matlab}

Artificial neural networks are powerful tools for supervised classification problems. In this section of the dissertation, a flexible neural network is constructed in MATLAB to classify the infants with regard to future apparent life-threatening events. The two goals will be to achieve high prediction accuracy while minimizing the number of relevant parameters. Compared with the neural network model in SAS Enterprise 
Miner, we have more control in the MATLAB model in terms of the learning algorithm and model parameters.

\subsubsection{Materials and methods}

The 5-min segments of HRV parameters of the 122 infants are used to build and test the neural network classifier. The popular three-layer feed-forward backpropagation network was chosen for our infant classification problem. The structure of the neural network must be determined first, which includes deciding on the number of hidden neurons and the activation function. There is no way to determine a good network structure only from the number of input and output neurons. The number of hidden neurons also depends on the size of training dataset and the complexity of the classification. Some papers offer rules of thumb for choosing the number of hidden neurons: (1) sum of the number of input and outputs, then divide by two; or (2) the number of training samples divided by 10 times the number of inputs [BER00]. However, such rules are not very reliable. We start with a rough topology of the network for our application, using 10 hidden neurons. The proper network structure can only be determined experimentally by comparing the performance of the network with varying structures on our specific dataset. The neural networks with different numbers of hidden neurons, ranging from 3 to 40, are tested. The best network structure will be chosen by comparing the prediction accuracy resulting from the different neural network structures.

The other important issue for the design of a neural network is to choose a proper learning algorithm. It has been shown that the neural network using the back propagation (BP) learning algorithm provides an effective way to solve many difficult problems. 
Therefore, the most widely used BP algorithm is chosen for our neural network, due to its good performance for the classification problem. However, rather than the standard BP algorithm, the Levenberg-Marquardt backpropagation train technique is used. LevenbergMarquardt (LM) algorithm, a standard nonlinear least squares fitting method, is an enhanced error backpropagation technique to increase training speed [NOC99].

When updating the weights of the neural network, the objective is to minimize the sum of the squares of the differences between the predicted response $\left(\hat{y}_{i}\right)$ and the true output value $\left(y_{i}\right)$ at each of the $\mathrm{n}$ data points.

$$
\min \sum_{i=1}^{n}\left(y_{i}-\hat{y}_{i}\right)^{2}
$$

The LM algorithm, a modification of the Gauss-Newton methods, solves the above problem through the use of a Gauss-Newton trust-region method. Its intent is to approach second-order training speed by avoiding computation of the Hessian matrix. When a sum of squares based performance function is used, the Hessian matrix can be approximated by: $H=J^{T} J$, and the gradient vector is $g=J^{T} e$, where $\mathrm{J}$ is the Jacobian matrix, and e is a vector of network errors. $\mathrm{J}$ and e are defined as follows:

$$
J=\left[\begin{array}{cccc}
\frac{\partial e_{11}}{\partial w_{1}} & \frac{\partial e_{11}}{\partial w_{2}} & \cdots & \frac{\partial e_{11}}{\partial w_{N}} \\
\frac{\partial e_{21}}{\partial w_{1}} & \frac{\partial e_{21}}{\partial w_{2}} & \cdots & \frac{\partial e_{21}}{\partial w_{N}} \\
\vdots & \vdots & \cdots & \vdots \\
\frac{\partial e_{M 1}}{\partial w_{1}} & \frac{\partial e_{M 1}}{\partial w_{2}} & \cdots & \frac{\partial e_{M P}}{\partial w_{N}}
\end{array}\right] \quad \text { and } \quad e=\left[\begin{array}{l}
e_{11} \\
e_{21} \\
\vdots \\
e_{M P}
\end{array}\right]
$$


where $\mathrm{N}$ is the number of weights, $\mathrm{M}$ is the number of outputs, and $\mathrm{P}$ is the number of patterns. The LM algorithm weight updating method is as follows:

$$
W_{k+1}=W_{k}-\left(J_{k}^{T} J_{k}+\mu I\right)^{-1} J_{k}^{T} e
$$

where $\mathrm{e}$ is the cumulative error vector on outputs, $\mathrm{I}$ is identity unit matrix, $\mu$ is a learning parameter, and $\mathrm{J}$ is the Jacobian matrix. The learning rate $\mu$ is automatically adjusted at each iteration to guarantee convergence. Actually, the LM algorithm performs a small step initially, and larger steps along the steepest descent direction and tends to take more efficient steps to approaching the minimum.

Besides the Levenberg-Marquardt training function, the gradient descent with momentum weight learning function is also applied, which calculates the weight change of a neuron from its input and error.

$$
\Delta W_{k+1}=\alpha \Delta W_{k}+(1-\alpha) \cdot \mu \cdot J^{T} e
$$

where $\alpha$ is the momentum, $\mu$ is the learning rate, and $e$ is the error.

The logarithmic sigmoid transfer function, $\log \operatorname{sig}(x)=\frac{1}{1+e^{-n}}$ is chosen as the link function for all of the neurons, after it was compared with the hyperbolic tangent sigmoid transfer function, and linear transfer function.

Different performance functions, including mean squared error, mean squared error with regularization performance function, and sum squared error, are used to measure the performance of the training process. The mean squared error with regularization performance function, which uses the weighted sum of the mean squared error and the mean squared weights and biases, provides the best performance for our model. 
Besides determining the neural network structure and parameters, it is very important to choose appropriate input variables to build the neural network classifier. Too many inputs can prevent the neural network from converging on a good model during training. In the first step to construct the neural network model, we use the following HRV inputs, including Median, SDNN, RMSSD, total power, LF/HF, HFW, LFW, ApEn, Poincare, fractal dimension, REM duration, REM/total sleep-wake, SDANN, SDNNIDX, and sleep state code. Then, we try to reduce the number of inputs according to the results from our correlation study and t-test to find the most efficient model. The results from the above models will be compared.

\subsubsection{Results}

Neural networks of different structures have been tested in this section of the dissertation. Sensitivity, specificity, misclassification rate of the models in classifying each 5-min segment of the infants, as well as classifying infants are used to evaluate the models. Relative operating characteristic (ROC) curves are used to visually compare the effect of different thresholds on the best model.

In the first step of constructing the neural network model, we tested the effect of the number of hidden neurons in the classification results. Table 6.7 shows the results in classifying all 5-min segments of the infants, while table 6.8 shows the classification of infants for both training and testing datasets, with a chosen threshold. The results in table (a) are for the neural networks that use mean square error performance function and tangent sigmoid transfer function, while the table (b) shows the results for the neural 
networks that applied mean squared error with regularization performance function and logarithmic sigmoid transfer function.

Table 6.7 Results for classifying all 5-min segments of the infants

\begin{tabular}{|c|c|c|c|c|c|c|c|c|}
\hline \multirow{2}{*}{$\begin{array}{l}\text { Number } \\
\text { of hidden } \\
\text { neurons }\end{array}$} & \multicolumn{4}{|c|}{ Training Dataset } & \multicolumn{4}{|c|}{ Testing Dataset } \\
\hline & Sensitivity & Specificity & $\begin{array}{l}\text { Misclassi- } \\
\text { fication }\end{array}$ & MSE & Sensitivity & Specificity & $\begin{array}{l}\text { Misclassi- } \\
\text { fication }\end{array}$ & MSE \\
\hline 3 & 0.8226 & 0.8935 & 0.1175 & 0.0672 & 0.1108 & 0.7719 & 0.3386 & 0.2406 \\
\hline 6 & 0.8165 & 0.913 & 0.102 & 0.069 & 0.1108 & 0.79 & 0.3236 & 0.2191 \\
\hline 8 & 0.8639 & 0.9602 & 0.0548 & 0.0369 & 0.1572 & 0.8288 & 0.2835 & 0.2691 \\
\hline 10 & 0.9392 & 0.962 & 0.0416 & 0.0263 & 0.1985 & 0.7729 & 0.3231 & 0.406 \\
\hline 12 & 0.8967 & 0.9385 & 0.068 & 0.0432 & 0.0335 & 0.7863 & 0.3395 & 0.2819 \\
\hline 20 & 0.9976 & 0.9664 & 0.0287 & 0.0209 & 0.1495 & 0.6265 & 0.4533 & 0.5149 \\
\hline 30 & 1 & 0.9971 & 0.0025 & 0.0035 & 0.5 & 0.5432 & 0.464 & 0.5803 \\
\hline 40 & 1 & 0.9975 & 0.0021 & 0.0041 & 0.4021 & 0.6482 & 0.3929 & 0.6068 \\
\hline
\end{tabular}

(a) Neural networks using mean square error performance function and tangent sigmoid transfer function

\begin{tabular}{|c|ccc|ccc|}
\hline \multirow{2}{*}{$\begin{array}{c}\text { Number } \\
\text { of hidden } \\
\text { neurons }\end{array}$} & \multicolumn{3}{|c|}{ Training Dataset } & \multicolumn{3}{c|}{ Testing Dataset } \\
\cline { 2 - 6 } & Sensitivity & Specificity & Misclassification & Sensitivity & Specificity & Misclassification \\
\hline 3 & 0.582 & 0.7876 & 0.2443 & 0.5077 & 0.8883 & 0.1754 \\
6 & 0.3949 & 0.885 & 0.1912 & 0.4485 & 0.9834 & 0.106 \\
8 & 0.6343 & 0.7843 & 0.239 & 0.4897 & 0.9121 & 0.1586 \\
10 & 0.5541 & 0.8378 & 0.2063 & 0.4046 & 0.9276 & 0.1598 \\
12 & 0.4678 & 0.8539 & 0.2062 & 0.4459 & 0.9581 & 0.1275 \\
20 & 0.5492 & 0.857 & 0.1909 & 0.4407 & 0.9312 & 0.1508 \\
30 & 0.6987 & 0.8494 & 0.174 & 0.3582 & 0.8453 & 0.2361 \\
40 & 0.7145 & 0.8306 & 0.1875 & 0.4381 & 0.8464 & 0.2219 \\
\hline
\end{tabular}

(b) Neural networks using mean square error with regularization performance function and logarithmic sigmoid transfer function 
Table 6.8 Results for classifying all of the infants

\begin{tabular}{|c|ccc|ccc|}
\hline $\begin{array}{c}\text { Number of } \\
\text { hidden } \\
\text { neurons }\end{array}$ & \multicolumn{3}{|c|}{ Training Dataset } & \multicolumn{3}{c|}{ Testing Dataset } \\
\cline { 2 - 7 } & Sensitivity & Specificity & Misclassification & Sensitivity & Specificity & Misclassification \\
\hline 3 & 0.9286 & 0.7887 & 0.1882 & 0.3333 & 0.7097 & 0.3514 \\
6 & 1 & 0.8451 & 0.1294 & 0.1667 & 0.7097 & 0.3784 \\
8 & 1 & 0.9437 & 0.0471 & 0.1667 & 0.6774 & 0.4054 \\
10 & 1 & 0.9014 & 0.0824 & 0.3333 & 0.6452 & 0.4054 \\
12 & 1 & 0.9577 & 0.0353 & 0.1667 & 0.4839 & 0.5676 \\
20 & 1 & 0.9296 & 0.0588 & 0.3333 & 0.3548 & 0.6486 \\
30 & 1 & 1 & 0 & 0.6667 & 0.2258 & 0.7027 \\
40 & 1 & 1 & 0 & 0.8333 & 0.3871 & 0.5405 \\
\hline
\end{tabular}

(a) Neural networks using mean square error performance function and tangent sigmoid transfer function

\begin{tabular}{|c|ccc|ccc|}
\hline \multirow{2}{*}{$\begin{array}{c}\text { Number of } \\
\text { hidden } \\
\text { neurons }\end{array}$} & \multicolumn{3}{|c|}{ Training Dataset } & \multicolumn{3}{c|}{ Testing Dataset } \\
\cline { 2 - 7 } & Sensitivity & Specificity & Misclassification & Sensitivity & Specificity & Misclassification \\
\hline 3 & 0.7143 & 0.8169 & 0.2 & 0.5 & 0.871 & 0.1892 \\
6 & 0.4286 & 0.9014 & 0.1765 & 0.5 & 1 & 0.0811 \\
8 & 0.7143 & 0.7606 & 0.2471 & 0.5 & 0.9355 & 0.1351 \\
10 & 0.7143 & 0.8451 & 0.1765 & 0.5 & 0.9032 & 0.1622 \\
12 & 0.5 & 0.831 & 0.2235 & 0.5 & 0.9677 & 0.1081 \\
20 & 0.5714 & 0.8592 & 0.1882 & 0.5 & 0.9355 & 0.1351 \\
30 & 0.7143 & 0.8592 & 0.1647 & 0.5 & 0.8065 & 0.2432 \\
40 & 0.7143 & 0.8451 & 0.1765 & 0.5 & 0.8387 & 0.2162 \\
\hline
\end{tabular}

(b) Neural networks using mean square error with regularization performance function and logarithmic sigmoid transfer function

Consistent with the results from SAS Enterprise Miner, the training of the neural network is very successful, which means the network obtains enough information about the HRV of these two types of infants from the training data. The best results for the testing dataset is for the regression model with $50 \%$ sensitivity and $100 \%$ specificity. In many of the structures, the testing results are not acceptable, especially the sensitivity. One reason might be over fitting of the model. Too much detailed information of the training infants might be remembered by the network, which reduces its ability to generalize. 
The above results show that the model parameters affect the classification results. There are apparent differences in table (a) and (b). Even though the training performance when using mean square error performance function and tangent sigmoid transfer function is much better on the training set than using mean squared error with regularization performance function and logarithmic sigmoid transfer function, the latter choice improves the generalization performance of the network for the test set. It does not achieve a very high training accuracy; however, it improves the sensitivity and specificity for the testing dataset. Therefore, the mean squared error with regularization performance function and logarithmic sigmoid transfer function is chosen for the neural network to do further testing.

The number of hidden neurons is another important parameter for the neural network. For our specific infant classification problem, the results show the trend that the training procedure improves with the increasing number of hidden neurons, especially when the mean squared error performance function is used. However, the results for the testing dataset are either decreasing or showing no change. We choose 8 hidden neurons for the neural network for further testing.

Besides the number of hidden neurons, choosing appropriate input variables is also critical to the classification models. The information in the previous HRV correlation study and t-test are used to find the best inputs for the neural network. Statistically significant differences between event and control infant groups are found in these parameters for at least two sleep states, which might provide power for classifying the infants. The input variables were reduced to the following set: mean, SDNN, RMSSD, 
ApEn, Poincare, REM duration, REM/total sleep-wake, SDANN, SDNNIDX, PCA, and sleep state code. The rest of the neural network parameters are kept as the same.

Figure 6.10 shows the ROC curves for classifying 5 -min segments and infants for the reduced set of inputs. Although the sensitivity in classifying 5-min segments is low, the result for infant classification is improved. For the testing dataset, 50\% sensitivity and $100 \%$ specificity can be reached.

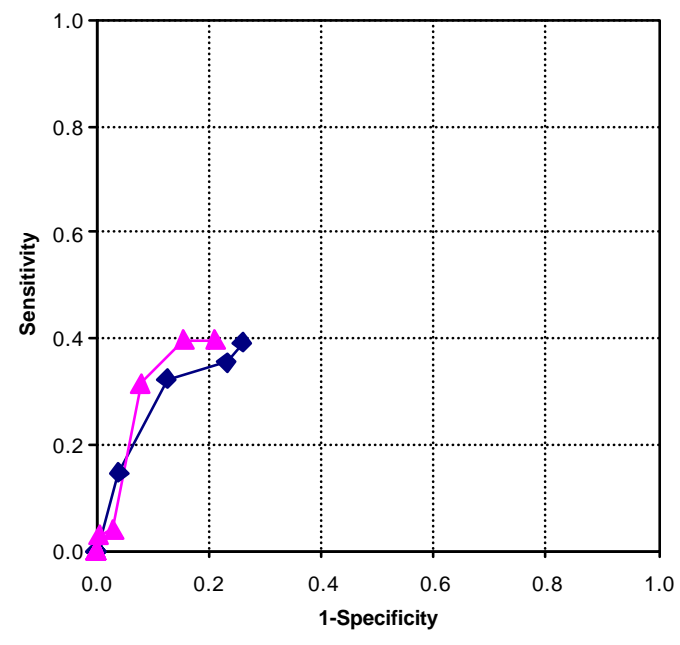

(a) Classifying all 5-min segments

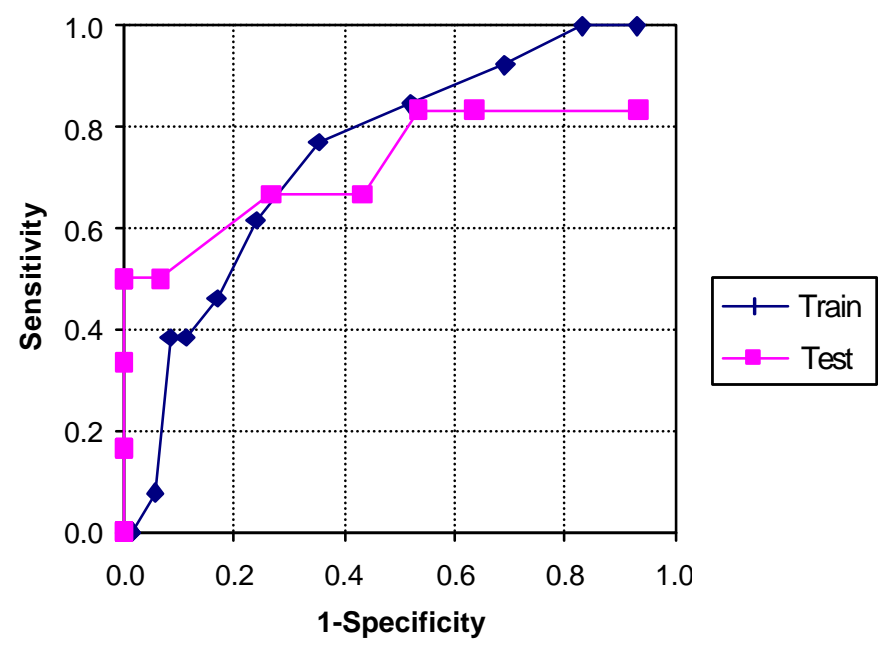

(b) Classifying all infants

Figure 6.10 Relative operating characteristic curve of neural network model for reduced input set

We have demonstrated that it is possible to achieve acceptable sensitivity and specificity with a particular arrangement of neural network. However, since the test set needed to be used to select the model which has the best generalization, it is unclear what the results would be for an untested dataset on one neural network model with one set of thresholds. It is possible that these results were achieved but are not reproducible on a separate dataset. While the results are not negative, there is not enough information to say 
that prediction is possible. In the next section, we explore reasons why prediction has been difficult in this dataset and attempt to address some of these considerations.

\subsection{Other Considerations}

While reasonable results were achieved by modifying the neural network structures, parameters, and learning techniques, it is not possible at this time to conclude that prediction is possible. There are several reasons we hypothesize for this which include size of the dataset and variability of the age of the infants. Insufficient data size might be the main reason for the difficulty in classification. The training data might not be enough to cover all the physiological characteristics of the infants, especially event infants. Furthermore, although there are 37 infants in the test dataset, only 6 of them are event infants. Even missing a couple of them will cause a dramatic drop in sensitivity.

In addition, the age when the PSG signal is taken was explored in Chapter 3. The results showed that the effect of age on heart rate variability is obvious (Figure 3.6). The difference in HRV due to age might be larger than the group differences. Unfortunately, the distributions of age for the event and control infants of our dataset are not similar at all. Age could be a very important input of the classification model. In our small dataset, age might affect the heart rate variability more than any other factors, particularly whether infants go on to have event. In this section, we explore the effect of age by including age as an input to the model, considering infants in a sub-group of ages, and age-matching each event infant with a control. 
Another aspect we explored was the separation of infants in the training and testing datasets. Since the test set is quite small, six event infants, it is possible that atypical infants are in the testing dataset.

\subsubsection{Effect of age}

The post conceptional age (PCA) of the infants when the polysomnogram (PSG) study was performed ranges from 34.3 to 62.9 weeks. It has been demonstrated in Figure 3.6 that the PCA of the infant affects the heart rate variability measurements. Therefore, the PCA of the infants is added as an input variable to the classification model and tested in this section.

For the 122 infants included in the classification study, the PCA is not available for two of the infants, 50150 (control infant) and 50153 (event infant). The rest 120 infants are used to construct and test the neural network classification model. There are 84 infants in the training dataset, and 36 infants in the testing dataset. The input variables now include: Mean, SDNN, RMSSD, HFW, LFW, LFW/HFW, ApEn, Poincare, fractal dimension, REM duration, REM/total sleep-wake, SDANN, SDNNIDX, PCA, and sleep state code. The structure of neural network is the same as in the last section, with 8 hidden neurons. Different thresholds for classifying 5-min segments and the infants are tested. The ROC curves in figure 6.11 show the results. 


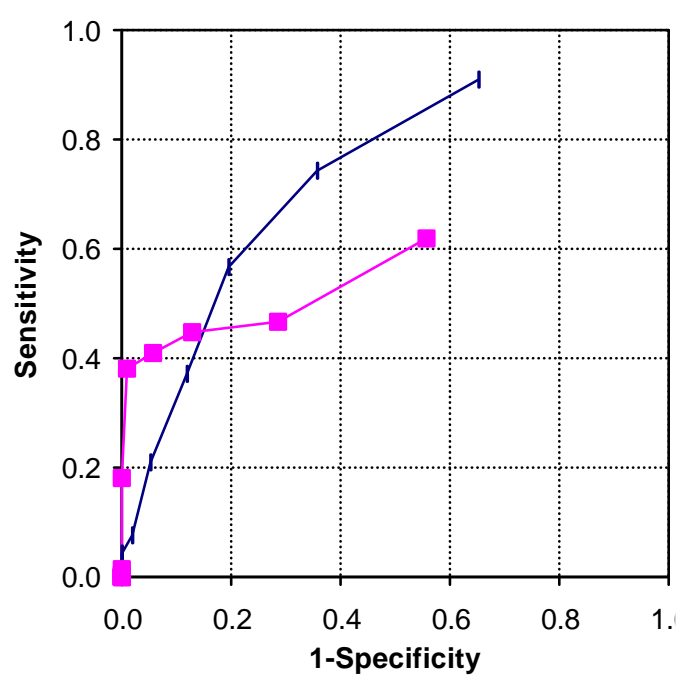

(a) Classifying all 5-min segments

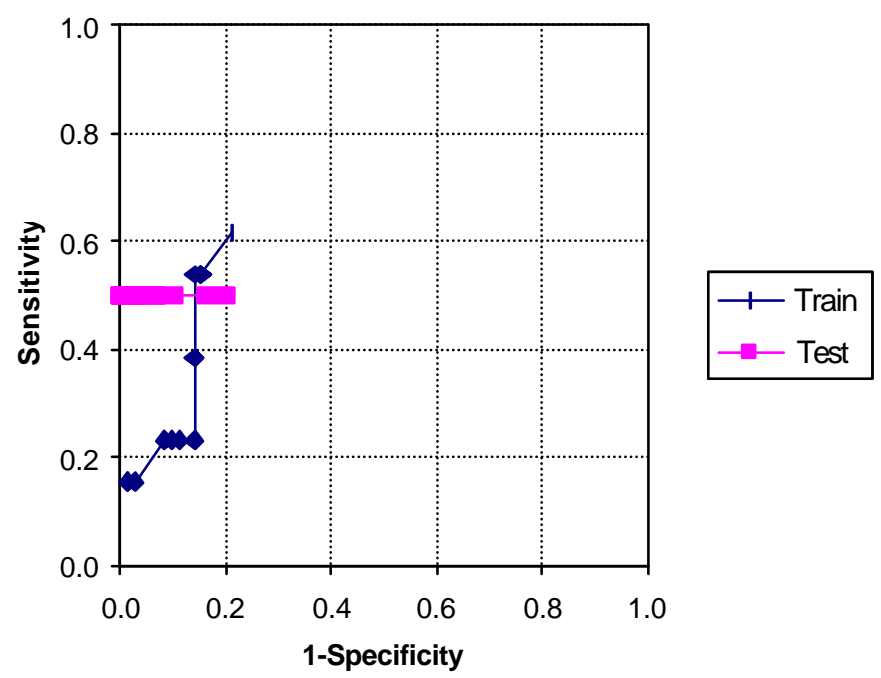

(b) Classifying all infants

Figure 6.11 Relative operating characteristic curve of neural network model with age as an input

The results show that the performance of the model in the infant classification does not change. For the testing dataset, the best results are $50 \%$ sensitivity and $100 \%$ specificity.

The three classification models in SAS Enterprise Miner are also tested after adding PCA as an input. In terms of classifying all 5-minute segments and infants, there is no improvement for the test dataset (See figure 6.12 and 6.13). 


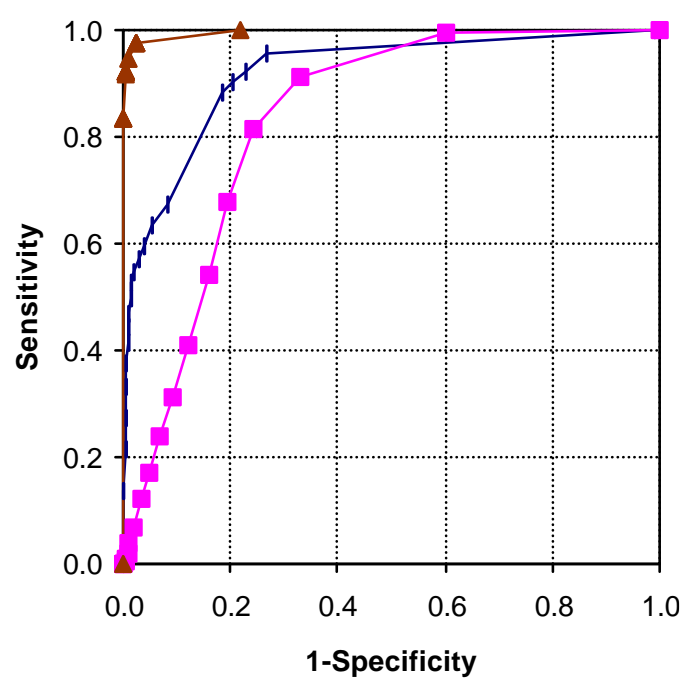

(a) Training dataset

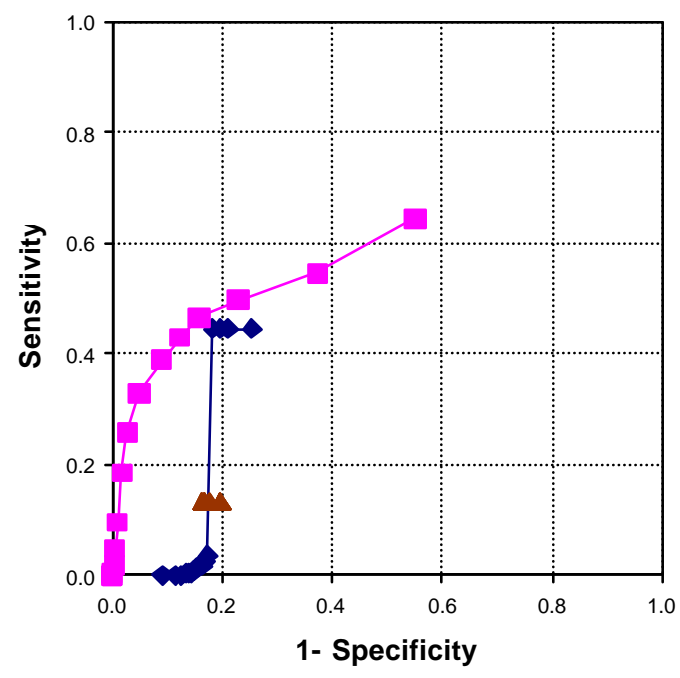

(b) Testing dataset

Figure 6.12 Relative operating characteristic curve of the models in classifying all 5-minute segments for training and testing sets

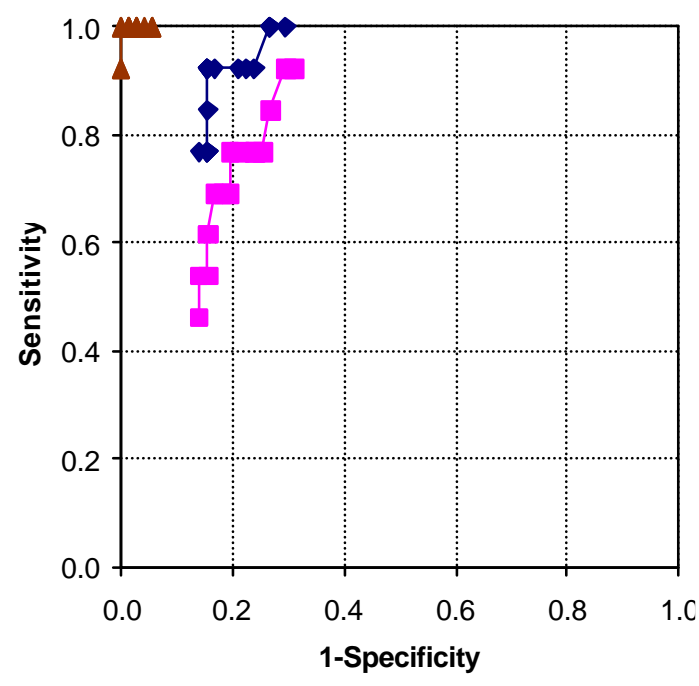

(a) Training dataset

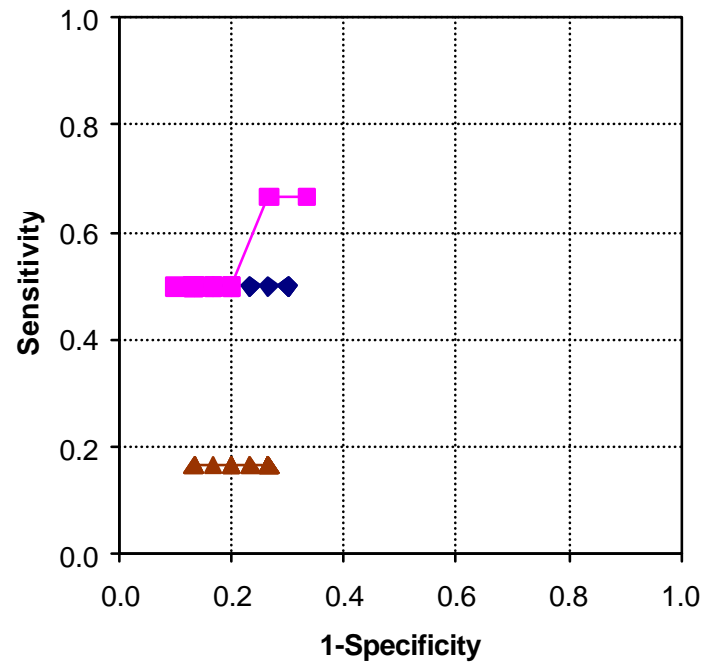

(b) Testing dataset

Figure 6.13 Relative operating characteristic curve of the models in classifying all infants in the training and testing sets 


\subsubsection{Utilization of age distribution information}

Since age is a large factor for the HRV measurements, several other options are considered in using such valuable information. For our classification model construction, the perfect situation would be to have the same age distribution for both event and control infants and enough infants at each age group, such that the age effect can be eliminated. However, this is not the case in our data. The age distribution plot in Chapter 3 showed that the age of the event infant is younger than the control infants. There are 18 out of 19 event infants with PCA from 35 to 45.6 weeks, but only 57 of the 101 control infants are in this age range. In order to minimize the influence of age in constructing the classification model, only infants with a PCA less than or equal to 45.6 weeks are used in this section to test the neural network. Therefore, all event infants except one are used. There are 54 and 21 infants in the training and testing datasets, respectively. Six of the 21 infants in the testing dataset are event infants.

Figure 6.14 shows the results. The performance of the neural network is improved in both 5-min segments and the infant classification. For the testing infant classification, $67 \%$ sensitivity is achieved while the specificity is still 100\%. Therefore 4 of the 6 event infants are captured and all the control infants are classified correctly. This result is very promising. 


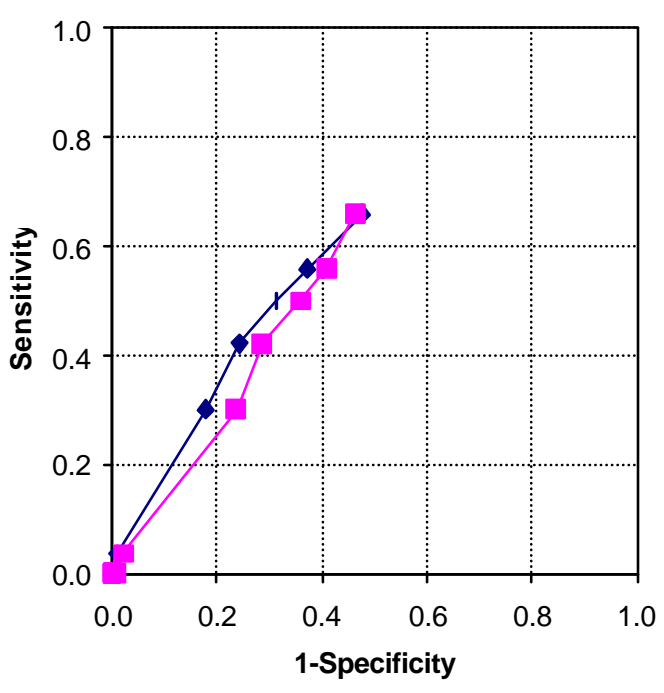

(a) Classifying all 5-min segments

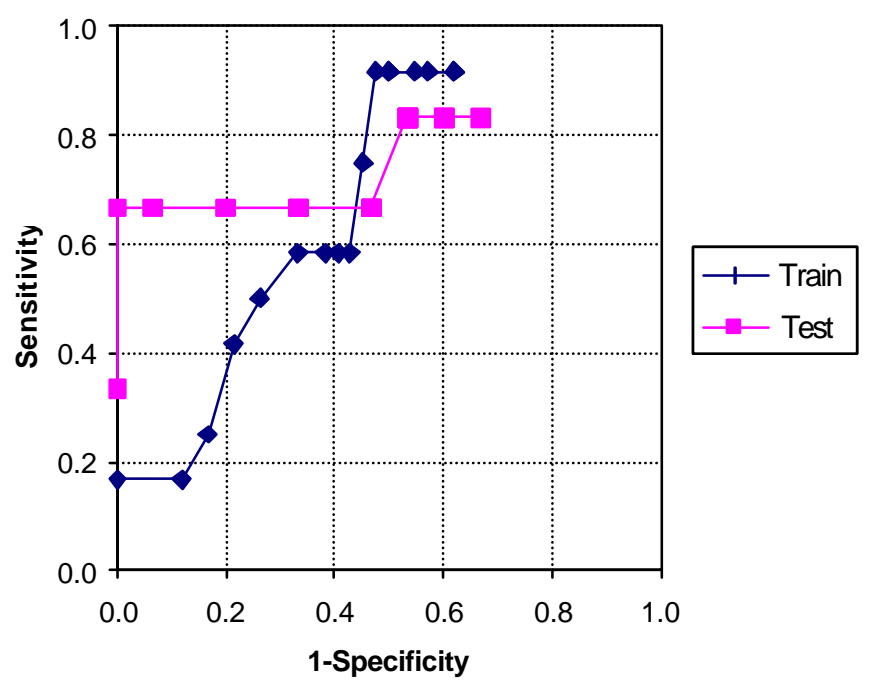

(b) Classifying all infants

Figure 6.14 Relative operating characteristic curve of neural network model for infants less than 45.6 weeks

\subsubsection{Age matching of the infants in event and control groups}

It has been shown that the generalization performance of the neural network was improved by adding the information of the age distribution in the last section. The age differences between the two groups of infant were reduced by excluding some control infants whose PCA are not in the common range of our event infants. To be more precise, this section matches event infants with controls in terms of age and group. Besides the event and nonevent classification of infants, which is based on the event recordings, the infant in the CHIME study are also categorized into seven groups, healthy (HT), preterm without symptom (PT-A), preterm with symptom (PT-S), term SIDS siblings (SIB-T), preterm SIDS siblings (SIB-P), term infants with apnea of infancy (AIO-T), and preterm infants with apnea of infancy (AIO-P). Each event infant is matched with one control infant from the same group and with the same or similar PCA. 
All of the event infants are from the preterm group, except two which are term SIDS siblings. Table 6.9 lists the PCA and group information of the 18 event and 41 control infants who have similar PCA to the event infants. Table 6.9 also indicates which control infants are chosen to match the event infants. Therefore, these 18 event and 18 control infants form a new dataset for statistical analysis and classification.

The same ttest as described in Chapter 3 is used to determine whether there are statistically significant differences between these event and control infants in the HRV parameters. The ttest is performed in different sleep states using the mean HRVs of the 5-min segments for each infant. Table 6.10 shows the resultant mean and standard deviation of the HRVs across 18 event and control infants. Similarly, no significant differences are found in any of the HRV parameters between the event and control infants, as well as REMtime and REMratio.

Even though the infants are age matched, the statistical analysis will not take into account the variability of the infants over the age range that is represented. Even though event infants had their PSGs performed between 35-46 weeks, this range is still quite large in terms of maturational changes in HRV. It is possible that a representative set of infants is needed for smaller age blocks in order to account for variability due to age. Furthermore, a more complex statistical model which incorporates age may provide more information.

Although no statistical differences are found, a neural network was tested for classifying the infants. To form the training and testing datasets, a $70 \%$ and $30 \%$ ratio is used. Therefore, there are 24 and 12 infants in the training and testing datasets, respectively. Six event infants are in the testing dataset. 
Table 6.9 Age and group information of event infants with matched controls

\begin{tabular}{|c|c|c|c|c|c|c|}
\hline \multicolumn{3}{|c|}{ Event } & \multicolumn{4}{|c|}{ Matched control } \\
\hline infantid & PCA & group & infantid & PCA & group & chosen \\
\hline 30108 & 35 & PT-S & 11070 & 35.1 & PT-A & $x$ \\
\hline 41078 & 37 & PT-A & 50106 & 36.9 & PT-A & $\mathrm{x}$ \\
\hline \multirow[t]{2}{*}{20086} & 37.7 & PT-S & 11053 & 37.6 & PT-A & \\
\hline & & & 11055 & 37.6 & PT-A & $\mathrm{x}$ \\
\hline 13056 & 38.3 & PT-A & 31047 & 37.9 & SIB-P & $\mathrm{x}$ \\
\hline \multirow[t]{2}{*}{11059} & 38.3 & PT-A & 41104 & 38 & PT-A & $x$ \\
\hline & & & 12055 & 38.7 & SIB-P & \\
\hline 11073 & 38.7 & PT-A & 13063 & 38.9 & PT-A & $x$ \\
\hline 20128 & 39.1 & PT-A & 13059 & 39.1 & PT-A & $\mathrm{x}$ \\
\hline 20111 & 39.3 & PT-S & 11064 & 39.3 & PT-A & $\mathrm{x}$ \\
\hline 11049 & 39.4 & PT-A & 24029 & 39.4 & PT-A & $x$ \\
\hline 11050 & 39.6 & PT-A & 20136 & 39.3 & PT-A & $\mathrm{x}$ \\
\hline \multirow{5}{*}{20098} & 40 & AOIP & 21004 & 40 & AOIP & $\mathrm{x}$ \\
\hline & & & 41090 & 40 & AOIT & \\
\hline & & & 13058 & 40.1 & PT-A & \\
\hline & & & 23011 & 40.1 & PT-S & \\
\hline & & & 20108 & 40.3 & PT-A & \\
\hline \multirow[t]{4}{*}{41074} & 40.6 & PT-A & 11063 & 40.6 & PT-A & $\mathrm{x}$ \\
\hline & & & 24018 & 41.6 & PT-A & \\
\hline & & & 24023 & 40.4 & AOIP & \\
\hline & & & 20108 & 40.3 & PT-A & \\
\hline \multirow[t]{2}{*}{20090} & 40.9 & PT-S & 20123 & 40.9 & PT-A & $\mathrm{x}$ \\
\hline & & & 13067 & 41 & PT-A & \\
\hline \multirow[t]{2}{*}{55012} & 41.1 & PT-S & 13060 & 41.1 & PT-A & $\mathrm{x}$ \\
\hline & & & 20144 & 41.1 & AOIT & \\
\hline \multirow[t]{5}{*}{11074} & 43.4 & PT-A & 23022 & 43.4 & PT-A & $\mathrm{x}$ \\
\hline & & & 50072 & 43.4 & PT-A & \\
\hline & & & 50095 & 43.6 & SIBP & \\
\hline & & & 10012 & 43.6 & AOIT & \\
\hline & & & 13055 & 44.7 & SIBT & \\
\hline \multirow[t]{2}{*}{31038} & 44.9 & SIB-T & 20102 & 44.7 & SIBT & $x$ \\
\hline & & & 12059 & 44.9 & SIBP & \\
\hline \multirow[t]{6}{*}{11052} & 45.1 & PT-A & 22006 & 45.1 & SIB-P & $x$ \\
\hline & & & 55013 & 45.1 & SIBT & \\
\hline & & & 50100 & 45.1 & AOIT & \\
\hline & & & 12060 & 45 & SIBP & \\
\hline & & & 50099 & 45 & AOIT & \\
\hline & & & 12067 & 45.4 & SIB-T & \\
\hline \multirow[t]{3}{*}{12044} & 45.6 & SIB-T & 31043 & 45.7 & SIB-T & $x$ \\
\hline & & & 30124 & 46 & $\mathrm{HT}$ & \\
\hline & & & 12054 & 46 & SIB-T & \\
\hline
\end{tabular}




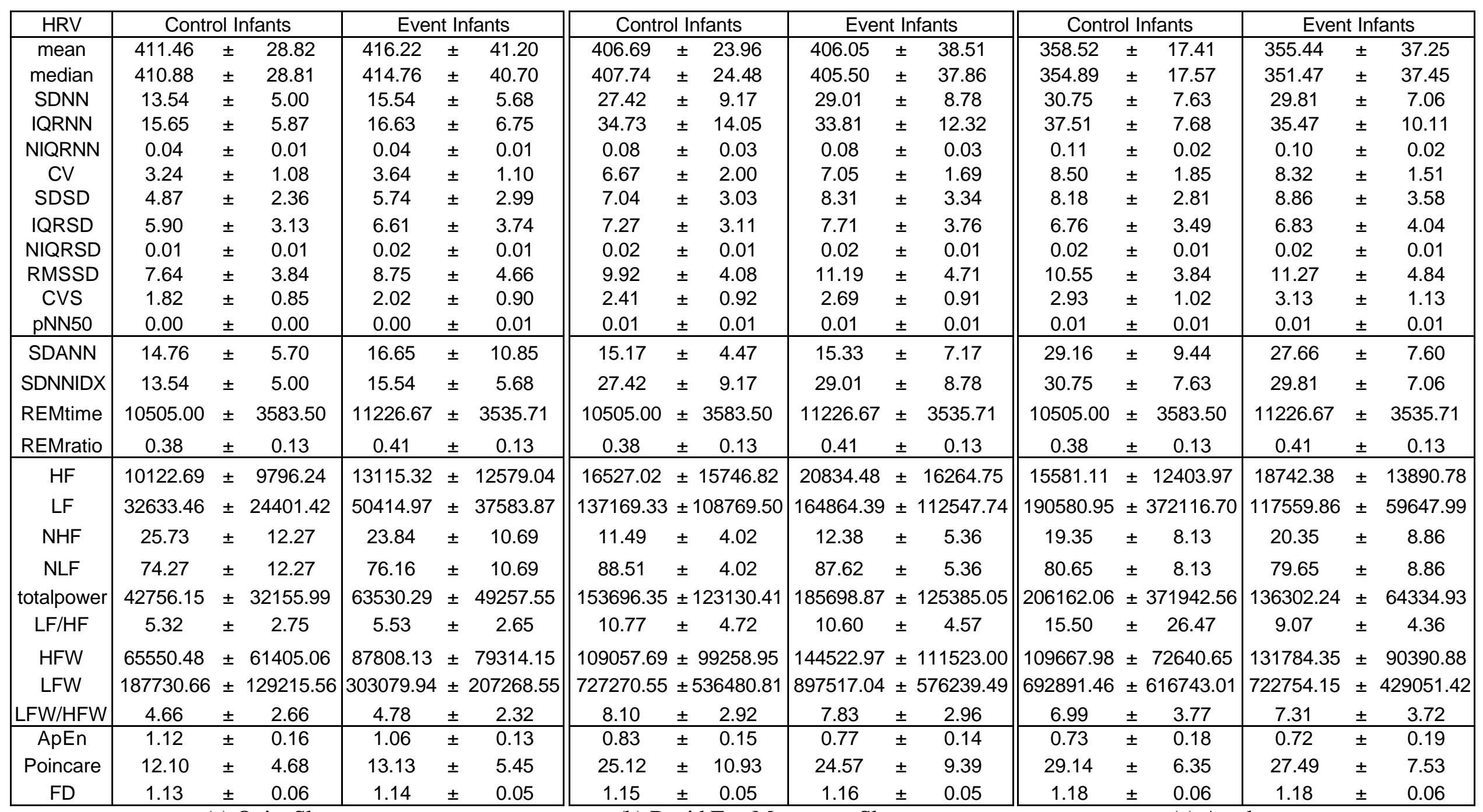

(a) Quiet Sleep

(b) Rapid Eye Movement Sleep

(c) Awake

Table 6.10 HRV measurement for the 36 age matched event and control infants 
As expected from the statistical analysis, the classification result is not improved over other tests with $50 \%$ sensitivity and $90 \%$ specificity as the best results for infant classification of the testing dataset, see figure 6.15 .

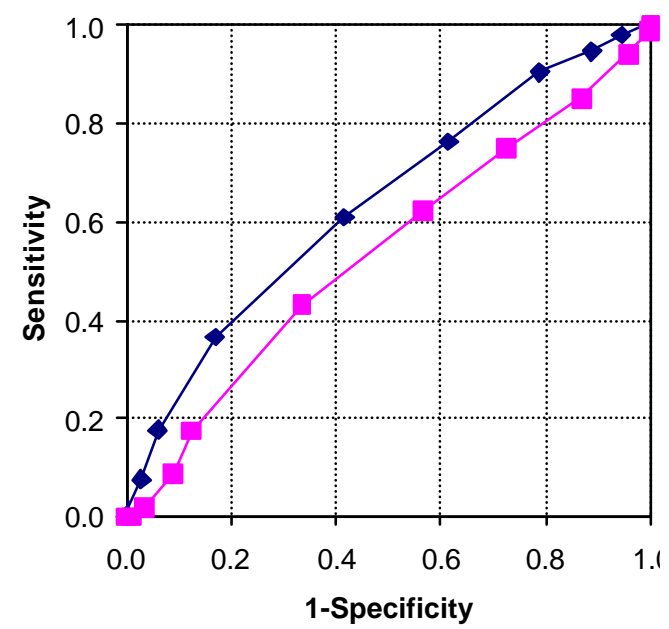

(a) Classifying all 5-min segments

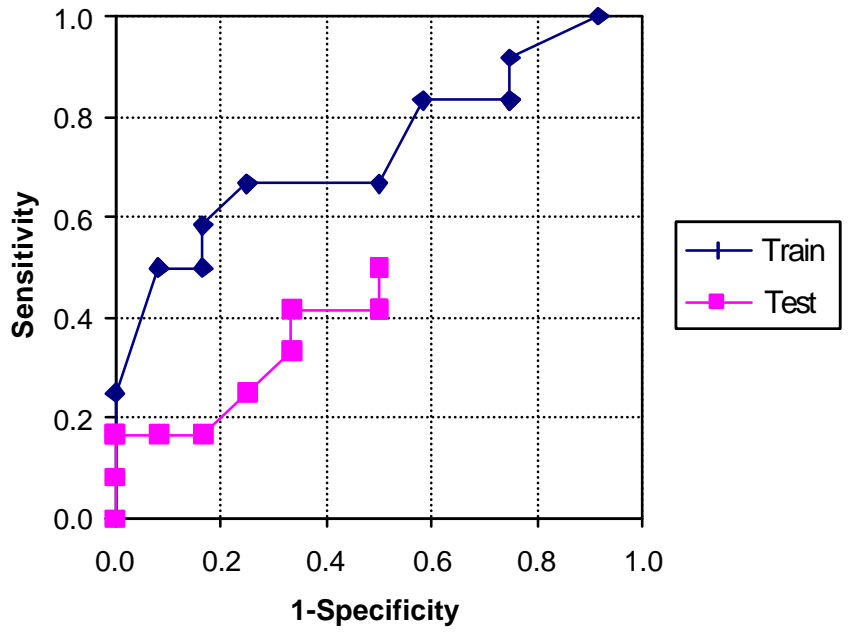

(b) Classifying all infants

Figure 6.15 Relative operating characteristic curve of neural network model for age matched control and event infants

\subsubsection{Re-randomize the training and testing datasets}

Since the data size for the classification study is very small (only 6 event infants in the test dataset), and all the above models except the last one are constructed and tested on the same randomized training and testing datasets, the data was re-randomized to create new training and testing datasets to assess whether it is consistent with previous results.

Only infants with a PCA of less than or equal to 45.6 weeks are used in this study. There are 50 and 25 infants in the new training and testing datasets, respectively. Figure 
6.16 shows the results. The classification results on the testing dataset are similar to the results from the first dataset, with $40 \%$ sensitivity and $60 \%$ specificity.

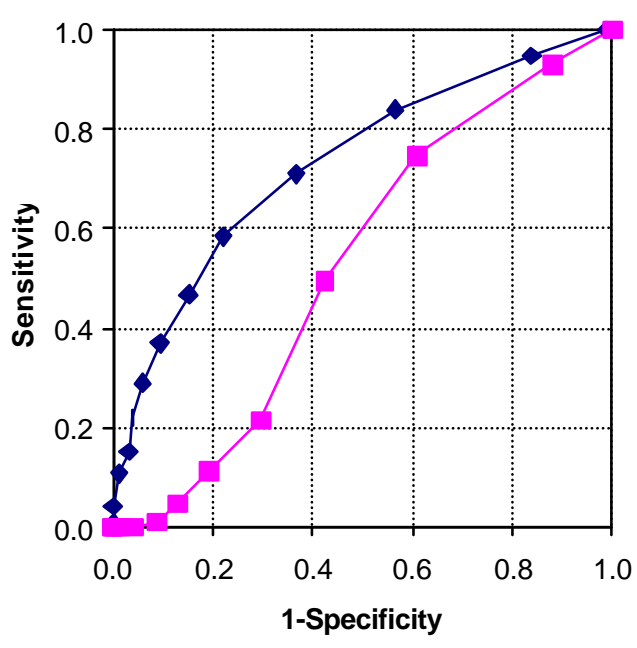

(a) Classifying all 5-min segments

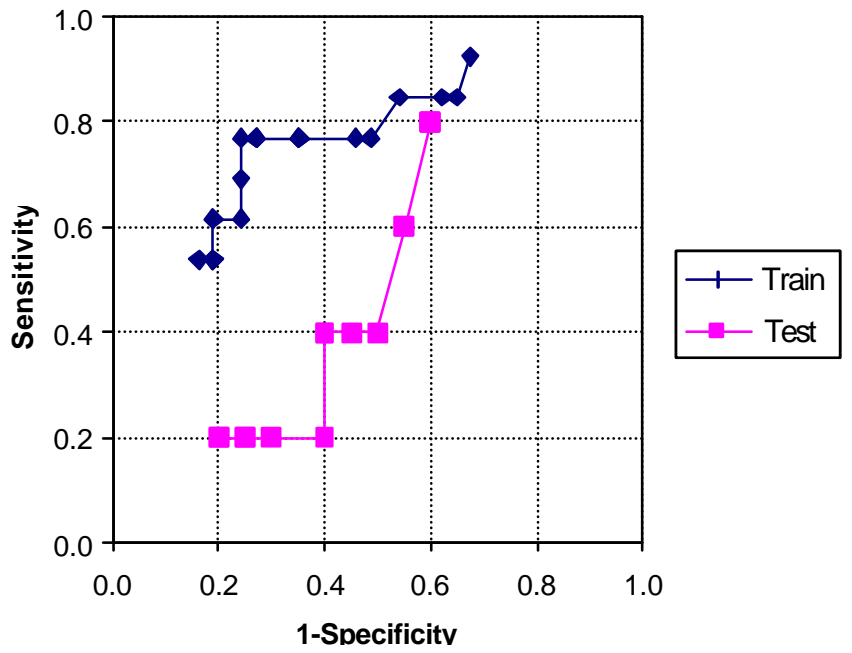

(b) Classifying all infants

Figure 6.16 Relative operating characteristic curve of neural network model for rerandomized training and testing datasets

\subsection{Summary}

The capability of using heart rate variability to classify event versus control infants is evaluated in this section of the dissertation. The supervised classification models, logistic regression, decision tree and neural network provided by the SAS Enterprise Miner, as well as the neural network model in the MATLAB toolbox are used to investigate the possibility of classifying event infants from the control. Although acceptable sensitivity and specificity of the testing dataset are achieved by varying the threshold, and network parameters, such results are not guaranteed for new dataset. A new validation dataset is needed to assess whether this accuracy can be achieved for an independent dataset. From those results, it is clear that age has an effect on classification. 
The main reason for unsuccessful classification might because the insufficiency of the data. More infants in each age group are needed to determine whether classification is possible.

Although the size of the dataset is too small for a classification or prediction study, various methods are tested in terms of whether it is possible to use them for the infant classification study infants. The result is promising. If more infant data are available, better classification results can be expected. 


\section{CHAPTER VII CONCLUSION AND FUTURE WORK}

Currently, there is not a means to predict which infants will go on to have lifethreatening events. The Collaborative Home Infant Monitoring Evaluation (CHIME) study group was developed to study the ability of home monitors to detect lifethreatening events of bradycardia and apnea. As part of this study, a polysomnogram (PSG) study was performed initially to assess the infant's sleep pattern including information regarding the cardiac system. We utilize heart rate variability calculated from the PSG to develop a means to predict infants at risk for future life-threatening events.

In order to accomplish this, we first developed and tested an artifact rejection routine to clean the raw $\mathrm{RR}$ interval data. Next we used correlation coefficients to determine the relationship of various HRV parameters and obtain more insight into HRV in order to use it properly in the classification study. Lastly, common supervised classification techniques, including logistical regression, classification trees, and neural networks, were applied to determine if infants which go on to have life-threatening events could be predicted. Successful prediction of at-risk infants could have clinical significance and provide physicians with an opportunity for treatment. 


\subsection{Conclusions}

\subsubsection{Artifact rejection}

A major consideration before analyzing heart period data or RR intervals is the removal of artifact. Automatic artifact rejection becomes critical when analyzing huge datasets, such as the CHIME dataset, where manual inspection is impossible. As the first study for this dissertation, an automatic artifact detection algorithm is developed based on the existing criteria, and compared with five other previous artifact rejection routines. The testing results for detecting both real and artificial artifacts showed that the new algorithm provides the best balance between sensitivity and specificity. Compared with the most popular algorithm, it greatly improves the specificity with only a slight loss in sensitivity. The new algorithm works extremely well for detecting missing and extra beats (100\% and $97.44 \%$ sensitivity, respectively), which are the most common artifact encountered in RR interval data. This algorithm is used to clean the RR intervals for further analysis.

In addition, the effect of artifacts on the heart rate variability measurements is studied, which emphasizes the importance and effectiveness of the automatic artifact rejection routine. This study indicates that, in addition to sensitivity, the specificity of the artifact rejection routines affect some HRV measurements seriously. False positives, and subsequent interpolation changes the RR interval data and resultant HRV measurements. This result demonstrates the importance of improving the specificity of artifact detection algorithms. The newly developed algorithm demonstrates its improvement over other algorithms by greatly improving the specificity. 
Sensitivity of the artifact rejection routines is also very important, especially the sensitivity to missing beats. Our results showed that even 2 missing beats in a 5 -min RR interval segment would produce a dramatic change in HRV. The newly developed algorithm achieved $100 \%$ sensitivity to missing beats even for noisy data. The slightly lower sensitivity of the algorithm is mainly due to the inability to detect some detection of type 4 and type 5 artifacts, noise where the QRS is discernable or undetectable, respectively. However, these two types of artifacts that were missed by our algorithm have only a small effect on HRV.

From studying the effect of artifacts on HRV, we suggest avoiding noise sensitive HRV parameters under noisy environments. Because no matter how good the artifact detection algorithms are, it is impossible to reach $100 \%$ sensitivity and specificity. Measurements which was least sensitive to noise include median, interquartile range, and approximate entropy.

\subsubsection{Correlation of HRV measurements}

Heart rate variability is used to study the difference between healthy control infants and infants with future apparent life-threatening event (FALTE) and classify them. However the relationship between numerous HRV parameters is unclear and the optimal parameters to use for infant classification are not known. When selecting measures for a specific application, an understanding of the relationship between HRV parameters is necessary in order to select the simplest methods and fewest numbers of necessary parameters. A systematic correlation study was performed between common HRV parameters in infants. A widely accepted highly correlated group of HRV that 
measures the vagal input is confirmed by our results. Some differences are noted compared to adult data. In addition, several rarely studied relationships, such as the correlation between nonlinear HRV parameters with others, are evaluated. Useful information is obtained, such as the approximate entropy (ApEn) is not correlated with any other HRV parameters. Such information provides guidelines in choosing inputs for our infant classification study.

\subsubsection{Prediction of life-threatening events}

The ultimate goal of this thesis is to predict apparent life-threatening event in infants using the PSG data. Popular supervised classification techniques, including logistic regression, decision tree, and neural networks, are utilized to construct the prediction model. Promising results $(67 \%$ sensitivity and $100 \%$ specificity $)$ were achieved for the test dataset where varying the model parameters and the threshold. $50 \%$ sensitivity and $100 \%$ specificity was a commonly reached performance in infant classification of the testing data; in other words, the model captured half of the event infants (3 infants) without a false alarm. Since our data size is very limited, only six event infants in the testing dataset, such results are not guaranteed for a new data. A new validation dataset is needed to assess whether this accuracy can be achieved for an independent dataset. If $50 \%$ sensitivity and $100 \%$ specificity can be consistently reached by independent infant datasets, the clinical importance of this study could be farreaching. Up until now, there is little information available for determining the likelihood of future life-threatening events for a newborn. If prediction at this accuracy level can be achieved through the measurement of HRV, half of the event infant population could be 
captured and assisted by offering corresponding therapy or extra health care. For such a prediction problem, high specificity is very important, because the number of normal healthy infants is much larger than the number of event infants. Many false alarms cannot be afforded. Future aims for classification will be to improve sensitivity without sacrificing much in specificity.

We conclude that although the size of the data is too small to make inference for a larger infant population, various methods are tested to determine whether it is possible to use them for infant classification. The results are promising. With more infant data, better classification can be expected and more confidence can be given to the results.

\subsection{Limitations of the Study and Future Work}

The main limitation for the classification study in this dissertation is the insufficiency of the data. The training data could not represent the physiological characteristics of the infants, especially event infants. Furthermore, although there are 37 infants in the test dataset, only 6 of them are event infants. With such a limited data size,

it is not possible to draw a confident conclusion in terms of whether the infant classification is successful or not. More data is needed to form an independent test set, and give a final unbiased score for the classification model.

From the results of our infant classification study, it is clear that age has an effect on classification. More infants in a narrow age range or more infants at each age from both the control and event groups are needed to construct and test the model in order to achieve better performance. 
Increasing the data size is the most important task for future work. Extracting the RR interval from the old format interval files is critical, which will increase our infant number by 131. Obtaining other missing information, such as the post conceptional age of the infant, or more PSG data, will also help to increase the size of our data. We can expect to have a dataset of approximately 350 infants, and up to 43 event infants.

With a larger dataset, more sophisticated statistical analysis can be performed, which incorporates both age and sleep state into the model. For classification, it will be possible to separate the dataset into three parts: training dataset, validation dataset, and testing dataset. The final scoring of the model on the holdout testing dataset provides a more reliable conclusion.

Since the number of control infants is much larger than that of event infants, some methods are needed for the training procedure to account for this. Given a larger dataset with more event infants, it is possible to randomly choose a similar number of control infants for the training, and repeat this procedure several times to build the best model. By using this method, sensitivity of the model is expected to be improved.

Furthermore, since the infants are classified based on any recorded events after the PSG. Events can occur as early as one day or as late as several months after the PSG. If more event infants are available, infants may need to be reclassified based only on events within a fixed window, for example, within one month or one week.

In addition, as was discussed in Chapter 4 , artifact must be removed or can greatly impact HRV parameters. Instead of using all 5-minute segments' data within the 8-hour PSG, selection and possible manual oversight of clean 5 -minute segments may be needed to build the classification model. 
Lastly, in the current design, sleep state is a separate input variable. It is possible that HRV parameters in different sleep states each contain different relevant information for making a prediction. For example, prediction might be improved if SDNN in REM and SDNN in QS are separate inputs in the model.

With this future work, improved classification results are expected. 
APPENDICES 
APPENDIX A

USER'S MENU FOR THE HEART RATE VARIABILITY ANALYSIS SOFTWARE 
A user-friendly interface was developed in MATLAB to calculate and analyze heart rate variability. Under MATLAB prompt, run program 'hrvgui.m', the following window will pop up as the main graphic interface of the software.

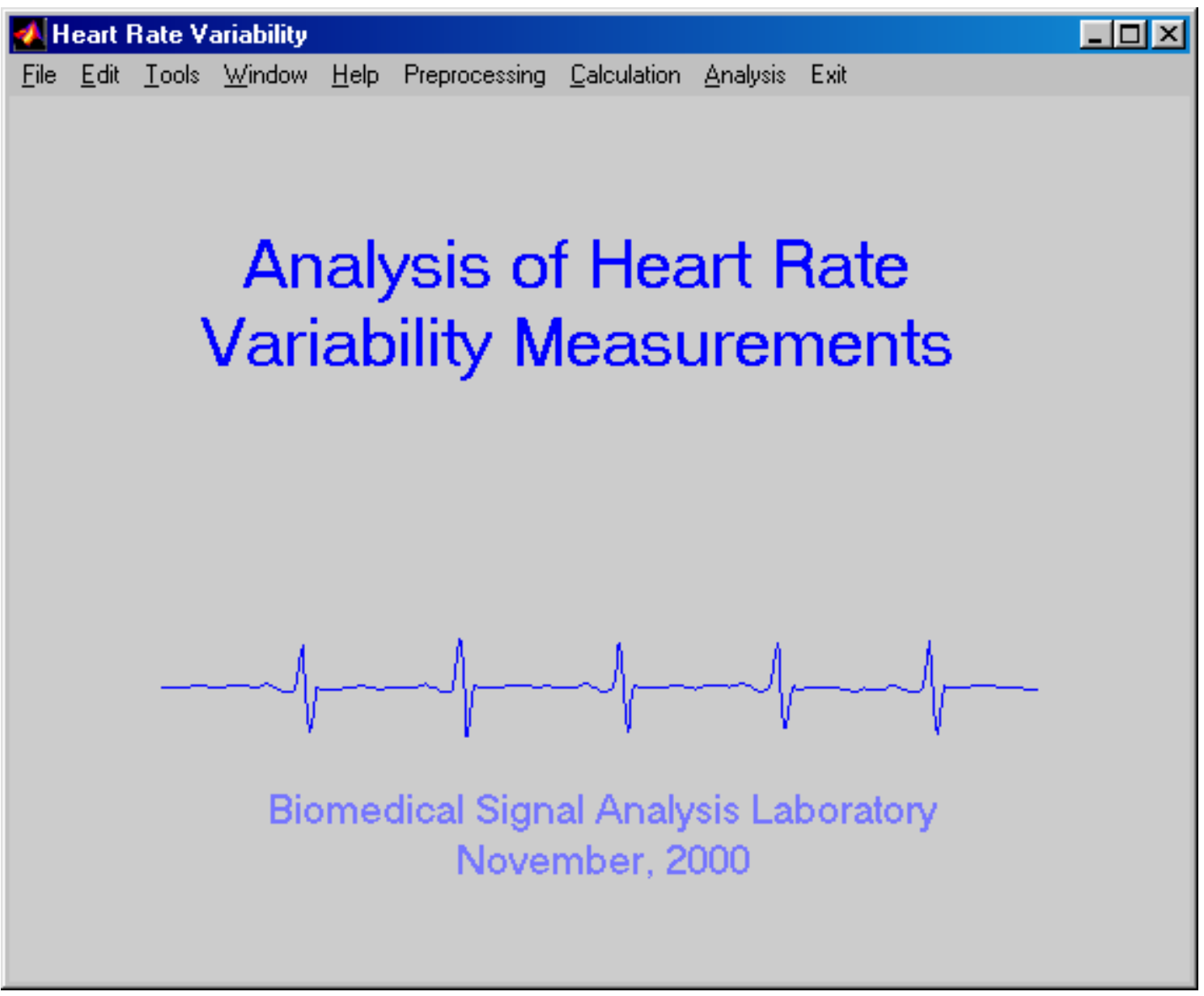

The software takes the original RR interval file as input. All of the following calculations and analysis are based on these raw data. There are three kinds of data processing which can be performed by this software, and are shown in the menu bar as Preprocessing, Calculation, and Analysis. Preprocessing contains Artifact Removal, Comparison of Artifact Detection Algorithms, and Effect of Artifacts on HRV 
Measurements. Artifact Removal uses our newly developed algorithm to detect artifacts and remove them by interpolation. Comparison of Artifact Detection Algorithms focuses on comparing previous and the new artifact detection algorithms in detecting fake missing and extra beats. Effect of Artifacts on HRV Measurements has functions for adding fake artifacts to clean RR intervals and investigating the effect of the fake artifacts (missing beats and extra beats) on heart rate variability calculation. The submenu under Calculation fulfills the task of calculating heart rate variability of 5-min $R R$ interval segments in the same sleep states, $x$-min RR interval segments in the same sleep states, and $\mathrm{x}$-min continuous $\mathrm{RR}$ intervals, where $\mathrm{x}$ can be selected. The analysis submenu contains plotting of the heart rate variability parameters for each individual infant and analyzing the changes of HRV with age and group differences. Each of these functions will be described one by one as follows. 


\section{Artifact Removal}

When you selected 'Artifact Remove' under 'Preprocessing' of the main menu bar, the following window will pop up.

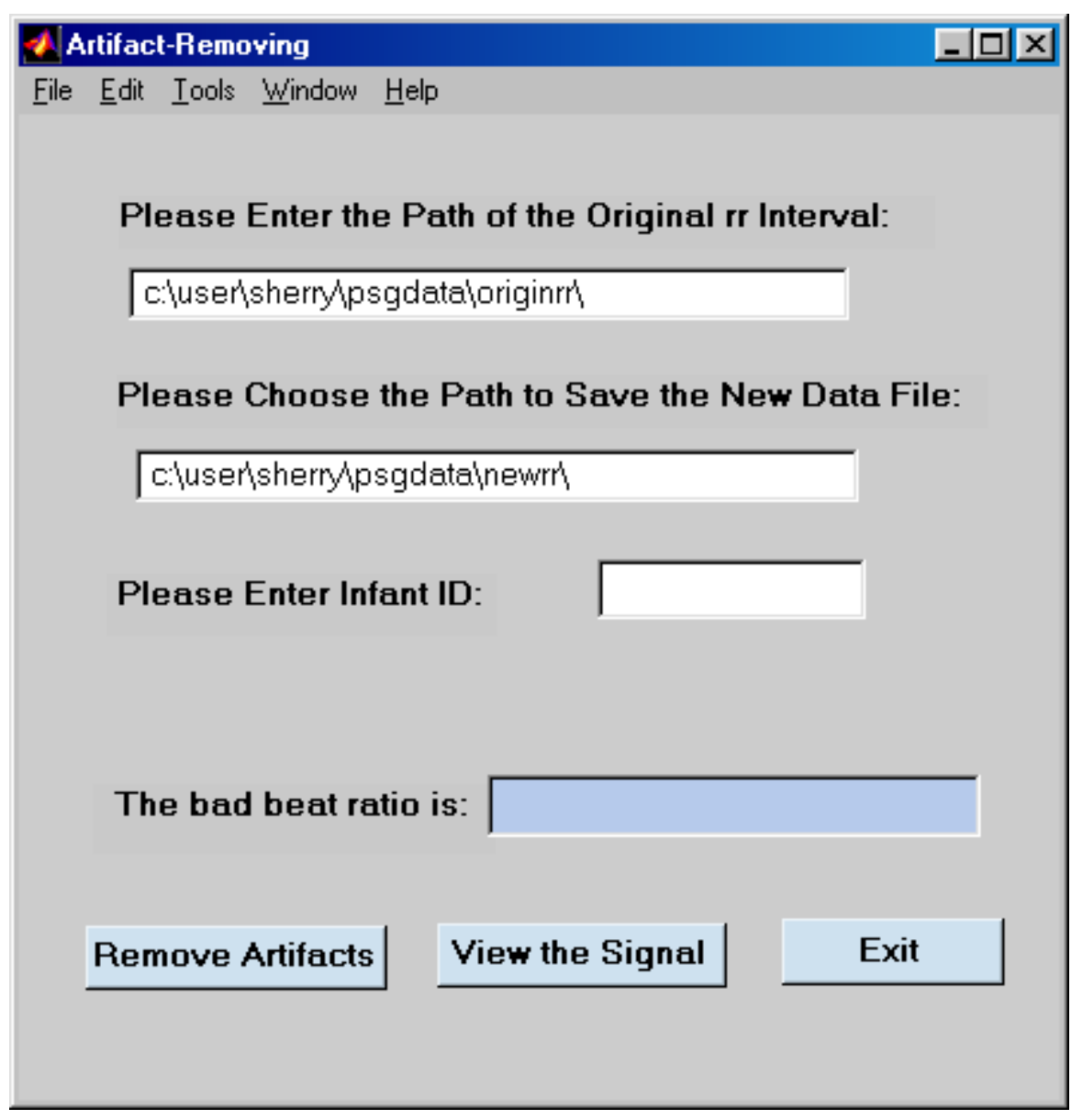

This program takes the original $\mathrm{RR}$ intervals as an input, which should be a vector of numerical data of instantaneous $\operatorname{peak}(\mathrm{R})$ to $\operatorname{peak}(\mathrm{R})$ time intervals in milliseconds. Then, it detects artifacts with our newly developed algorithm f. Cubic spline interpolation in MATLAB was used to correct the detected artifacts. The result contains original RR interval (x, rr), corrected RR intervals (X, RR), and a vector of bad beat information (bad_beat_flag, which marks every beat in RR with a 0 for good beats and 1 for bad 
beats) which are saved in a file. The file name is <infantIDRR.mat>, for example, 12058RR.mat.

When using this Artifact Removal interface to do the preprocessing of the data, you need to give the path of the original RR interval, the path $\mathrm{b}$ save the result, and the infant ID. There are default path shown in the edit box. All the calculation will be performed and the result will be saved after you click the 'Remove Artifacts' button. Then, the bad beat ratio, which is the number of interpolated RR over the total number of RR intervals will be shown in the last edit box. If this ratio is too high, the user can choose not to use that in the future analysis. The processing time for our RR intervals, which are approximately 8 -hour is about $52-58$ seconds on a Pentium III $600 \mathrm{MHz}$ computer. Clicking 'View the Signal' button, the following 'RR Interval Browser' window will popup, which allows the user to view the continuous RR interval signals minute by minute, hour by hour, or in its entirety. 


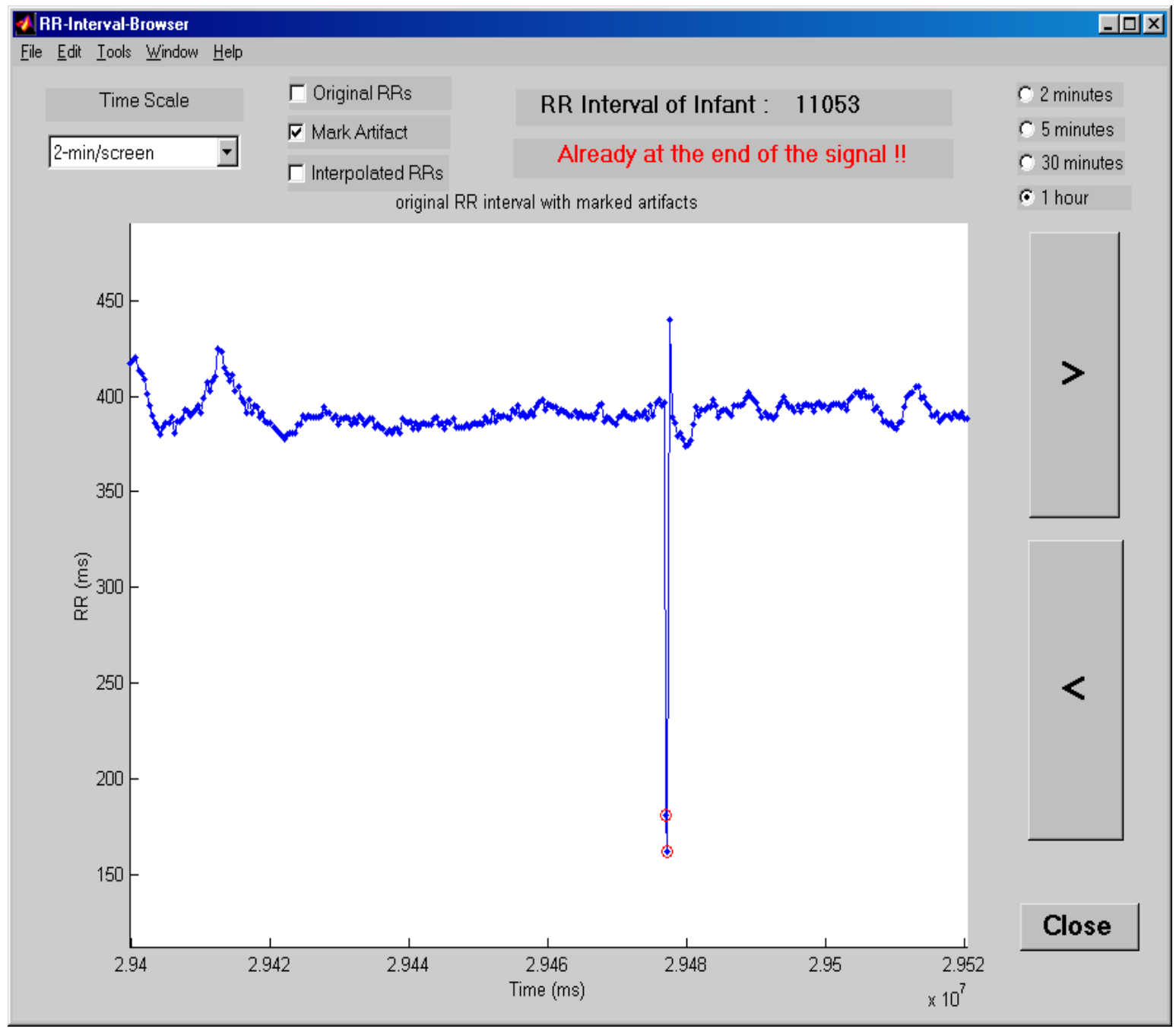

Using this GUI, you can choose to display the original RR interval signal, the original RR with artifacts marked by red circle, or the new RR interval after interpolation using the check box. The popup menu is used to select the time scale of displaying the signal on each screen. The radio buttons are used for choosing the step time to jump. The two text boxes are used for showing the infant ID, and give a warning when it goes to the end or the beginning of the signal. 


\section{Comparison of Artifact Detection Algorithms}

When you select 'Comparison of Artifact Detection Algorithms' under 'Preprocessing' of the main menu bar, the following window will pop up.
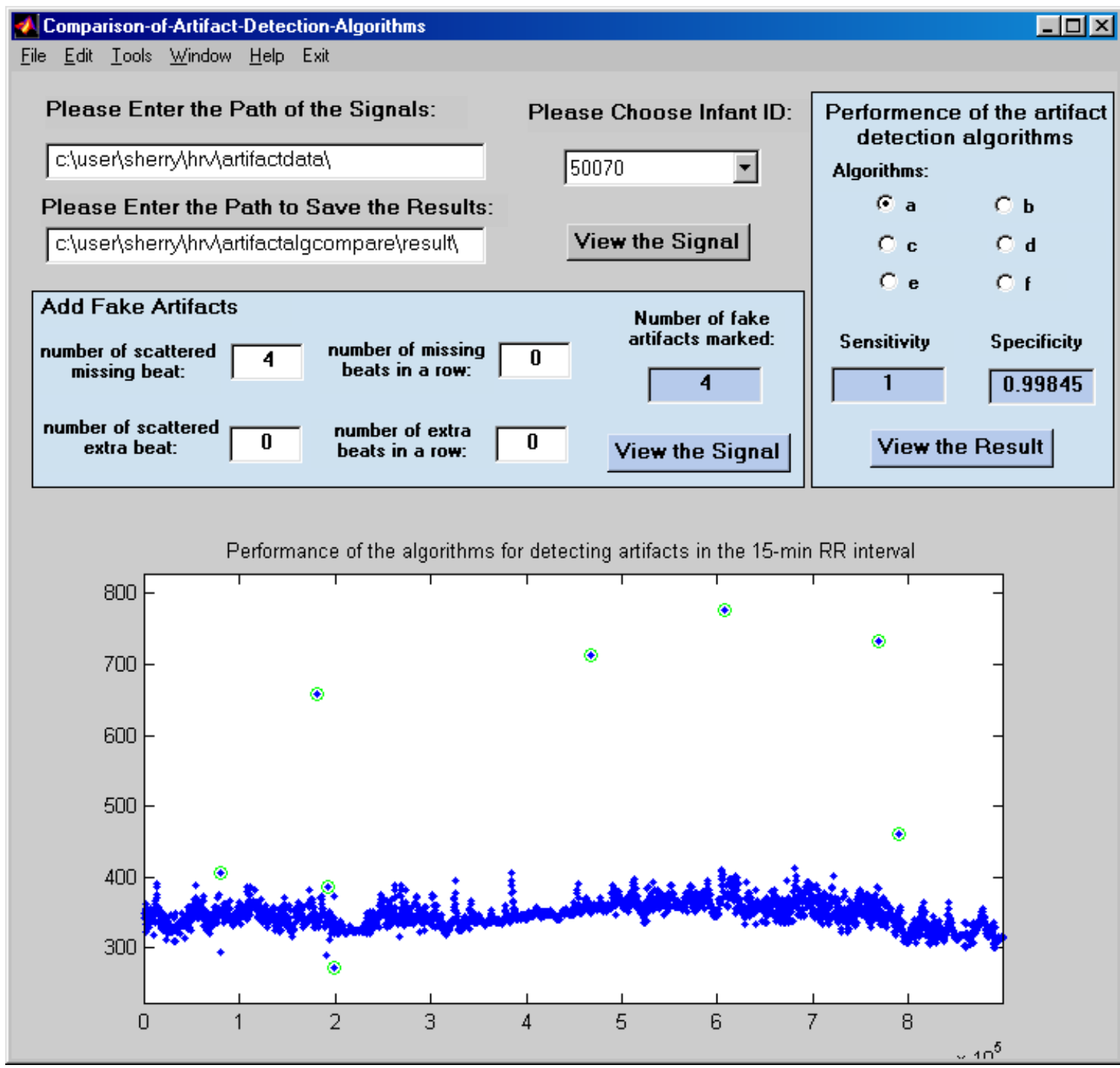

15 minutes RR intervals from 10 infants, which were chosen randomly are used to study the performance of each artifact detection algorithms in detecting fake artifacts. Given the path of the original data and the infant ID, the user can view the original 15min RR interval by clicking 'View the Signal' button under infant ID. The red circles indicate the real bad beats, which were marked by manual scanning of ECG. The second 
step is to add fake artifacts, including scattered missing/extra beats and missing/extra beats in a row. Fill the four editing boxes with the number of artifacts of each kind you want to add, the default value is zero. It allows the user to make a combination of different kinds of noise. When clicking 'View the Signal' button in the 'Add Fake Artifacts' Frame, the program checks the original signal first. If there are real artifacts in the original data, it will correct them by interpolation. Then fake artifacts are added into the cleaned RR interval data, and the signal with fake artifacts is displayed on the screen. Each of the added fake artifacts was circled red. The editing window will also tell the user the number of fake artifacts marked by the program. The last step of this program is to compare the performance of the six algorithms in detecting the added fake artifacts, which was fulfilled in the frame 'Performance of the artifact detection algorithms'. The radio button is used to choose an algorithm for testing. Only one algorithm can be chosen at a time. Clicking 'View the Result' button, the detection results of the selected algorithm will be shown on the screen. This time the green circle is the bad beat detected by the chosen algorithm. The performance of the algorithms can be compared visually through the resulting plots. Also, the sensitivity and specificity of each viewed signal will also be calculated and shown in the editing box on the screen. The comparison can be down quantitatively. If the original 15-min $R R$ intervals is used without adding fake artifacts, the performance of the algorithms can also be compared using the real artifacts in the original data. The red circles which are left in the figure are the undetected artifacts. Running initialcm.m first at the MATLAB command window, and then choosing the 10 infants one by one allows the user to calculate the overall sensitivity and specificity of the 10 infants. 


\section{Effect of Artifacts on HRV Measurements}

When you selected 'Effect of Artifacts on HRV Measurements' under 'Preprocessing' of the main menu bar, the following window will pop up.

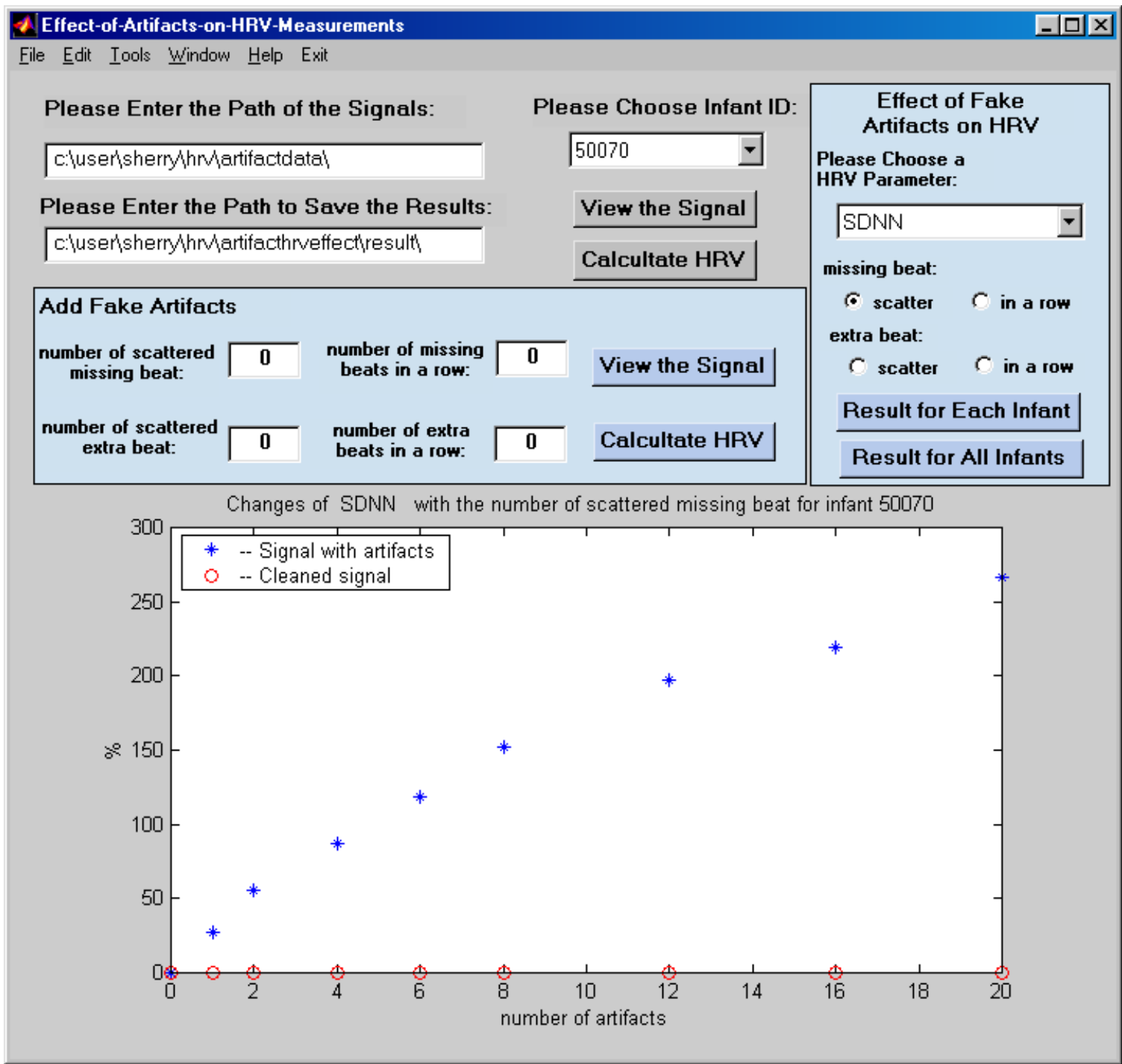

Since almost all the heart rate variability (HRV) calculation and analysis are based on consecutive 5 minutes segments of $R R$ interval in this project, the first 5 minutes of 15 minutes randomly chosen RR intervals from 10 infants were used to study 
the effect of the fake artifacts on the HRV parameters. HRV parameters are computed for the 5-min RR interval, where one value is gained for each HRV parameter per infant.

This window is very similar to the above window in the 'Comparison of Artifact Detection Algorithms' section. The first two parts, which were used to view the original signal and adding fake artifacts to the cleaned RR intervals, are exactly the same. The new function in these two parts is the calculation of HRV, which can be fulfilled by pressing the two 'Calculate HRV' button. The first 'Calculate HRV' button calculates all the HRV for the artifact-free RR intervals, it removes the real artifacts in the original RR by interpolation, and then computes the HRV. The second 'Calculate HRV' button in the frame 'Add Fake Artifacts' calculates HRV for both fake artifacts contaminated RRs and the RRs that cleaned using the newly developed artifact detection algorithm. The results will be saved into the given directory(c:luser|sherrylHRVlartifacthrveffect|result $\backslash 23020$ ), with the filename like 23020hrvorig.mat, 23020hrvnoisy6000.mat, respectively. 23020 is the infant ID, and 6000 means that is the HRV of RR interval with six scattered missing beats. The last four digits are used the mark number of fake artifacts: scattered missing, scattered extra beats, missing beats in a row, and extra beats in a row, respectively. Only missing beats and extra beats were considered, because those are the major artifacts in RR interval data. They are also considered to affect the HRV parameters the most compared with other kinds of artifacts.

The last step of this program is in the frame 'Effect of Fake Artifacts on HRV', which has the function of comparing the changes of HRV parameters with the number of artifacts. The program allows the user to compare all of the HRV parameters that were selected from the popup menu. Only one kind of artifact can be compared at a time, 
which must be given using the radio button. The comparison results of each infant can be shown on the screen by pressing the 'Result for Each Infant' button, which gives the user both the absolute values of HRV and the changes in percentage versus the number of artifacts. The results for each HRV parameter are also saved into a given directory (c:luserlsherrylHRVlartifacthrveffect|resultlall), with a name like SDNNsmiss23020.mat. The structure of result is shown in the following table, using SDNN as one of the HRV parameters.

\begin{tabular}{|c|c|c|c|c|}
\hline $\begin{array}{c}\text { Number of } \\
\text { artifacts(scattered } \\
\text { missing beats) }\end{array}$ & $\begin{array}{c}\text { SDNN for RR } \\
\text { with artifacts }\end{array}$ & $\begin{array}{c}\text { Changes of } \\
\text { SDNN (\%) }\end{array}$ & $\begin{array}{c}\text { SDNN for RRs after } \\
\text { removing artifacts by } \\
\text { algorithm } \mathrm{f}\end{array}$ & $\begin{array}{c}\text { Changes of } \\
\text { SDNN (\%) }\end{array}$ \\
\hline 0 & SDNN0 & 0 & SDNN0 & 0 \\
\hline 1 & SDNN1 & & SDNND1 & \\
\hline 2 & SDNN2 & & SDNND2 & \\
\hline$\ldots$ & $\ldots$ & $\ldots$ & $\ldots$ & $\ldots$ \\
\hline
\end{tabular}

Where SDNN1/2 are the absolute value of SDNN with one or two scattered missing beats, etc. The change in percentage is defined as:

$$
\frac{|S D N N 1-S D N N 0|}{S D N N 0} \times 100
$$

The 'Result of All Infants' button is used for calculation of the average percentage change of HRV over 10 infants for each HRV parameter. The result is a vector containing the HRV change in percentage of each infant, as well as the mean and standard deviation over 10 infants. 


\section{HRV Calculation}

When you select the submenu '5-min segments in the same sleep state' under 'Calculation' of the main menu bar, the following window will pop up.

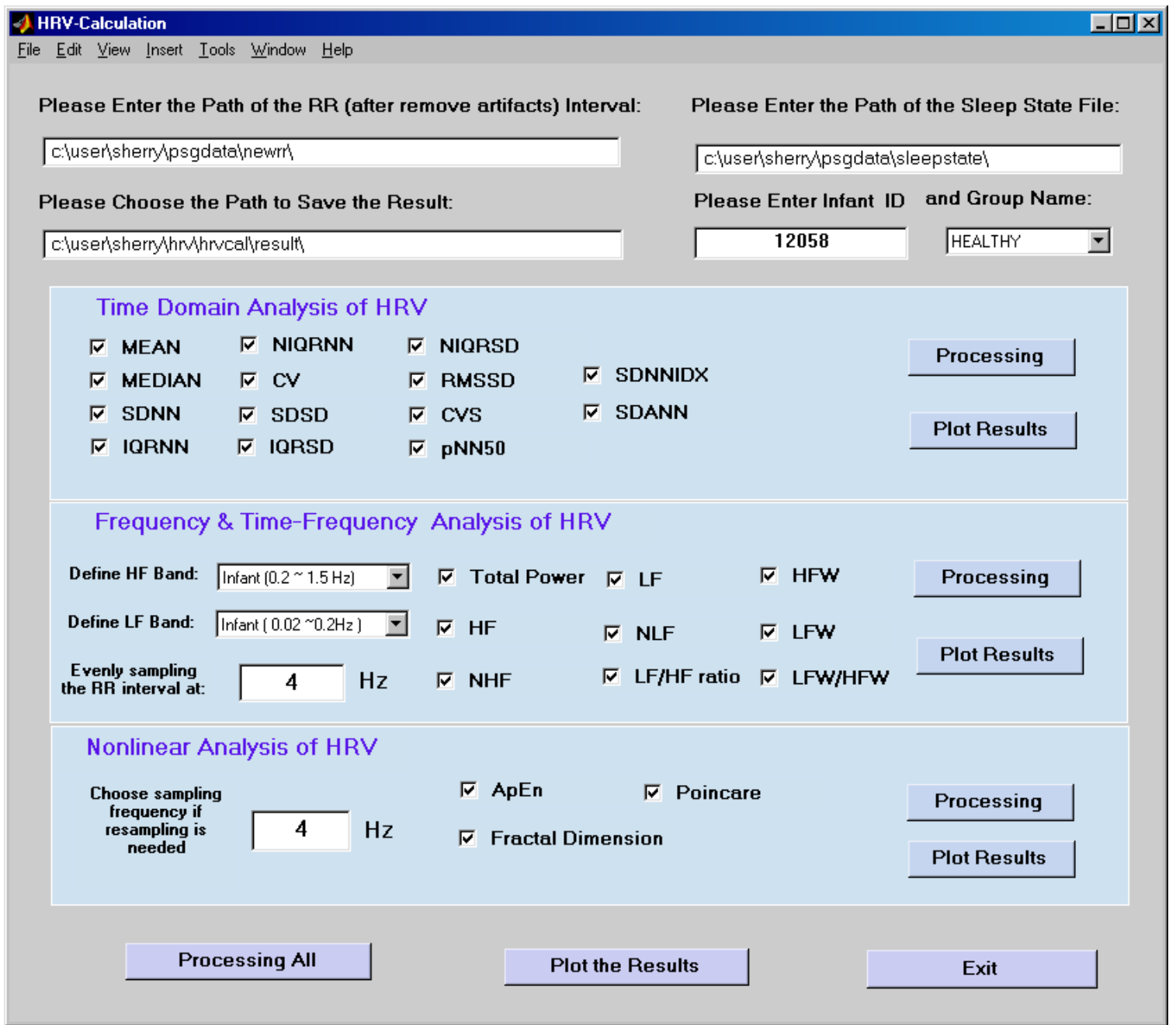

This program fulfills the function of calculating and saving all the most popularly used heart rate variability (HRV) parameters, which include time-domain, frequency domain, time-frequency domain, and nonlinear HRV measures, of all consecutive 5-min segments in the same sleep state of the 8-hour RR interval data for each infant. 
When using this interface to calculate HRV, the user needs to provide the path of the artifact-free RR interval, sleep state data, and the path for saving the results, as well as the infant ID and group name. The calculation and the result plotting can be done separately in time-domain, frequency-domain, or nonlinear-domain by pressing the 'Processing' or 'Plot Results' buttons in each section. The results will be saved separately. This work can also be done altogether by pressing 'Processing all' and 'Plot all Results' buttons. All the HRV parameters will be calculated and plotted altogether, but the results will still be saved for different domains separately.

The input data we use here are 8-hour continuous RR intervals with matched sleep state data. The sleep state file classified every 30 seconds of the RR interval into three sleep-waking states, quiet sleep (QS), rapid eye movement sleep (REM), awake (AWK), and indeterminate state. Since a 5-min window is used for the calculation of $\mathrm{HRV}$, the first step is to find 5 -min continuous RR intervals in the same sleep state from the first point. If there is 5 min recording in one sleep state, all HRV calculations can be done for that segment. The window is slide to keep on searching for other segments which satisfy the 5-minute sleep state criterion. If the criterion is not satisfied, the program slides one 30 second segment to find a 5 -min recording in one sleep state. There are three files, named RRQS, RRREM, and RRAWK, used to save these 5-min RR intervals for QS, REM, and AWK separately.

Fourteen time-domain HRV parameters can be calculated. The first three columns of HRV measures are calculated for each 5-min segment in each sleep state. The results are saved in a matrix per sleep state per infant. For the last column parameters, all 5-min segments in one sleep state are gathered together, and then the calculation is performed 
for HRV measures which have only one value per sleep state per infant, according to the definition of SDNNIDX and SDANN. The result is saved in the order of Mean, Median, SDNN, IQRNN, NIQRNN, CV, SDSD, IQRSD, NIQRSD, RMSSD, CVS, and pNN50.

Nine parameters are included in the frequency and time-frequency analysis of HRV in our program. The program is designed for HRV analysis of infant, adult, and rabbits. Since the definition of frequency bands is different for infant, adult, and animals, the user must select frequency band and sampling frequency first. Each 5-min segment of RR interval is resampled evenly at the chosen sampling frequency using cubic slpine interpolation. The default sampling frequency is $4 \mathrm{~Hz}$. For frequency analysis, Welch's averaged, modified periodogram method is used to estimate the power spectral density. Hanning window is applied in time-domain. The length of the window is half the length of the resampled RR intervals. Mean of the RR interval is subtracted as a kind of detrending procedure. Using the default values, we can get $0.0067 \mathrm{~Hz}$ frequency resolution for the evaluation of PSD. The total power here is defined as the sum of the low frequency power and the high frequency power. For the time-frequency analysis (wavelet), six Hertz sampling frequency is used. Daubechies 4 wavelet is chosen as mother wavelet, and the multiresolution pyramidal DWT algorithm is applied. The result of frequency domain parameters are saved in the order of HF, LF, NHF, NLF, total power, $\mathrm{LF} / \mathrm{HF}$ ratio, $\mathrm{HFW}, \mathrm{LFW}$, and $\mathrm{LFW} / \mathrm{HFW}$.

Three nonlinear measures of HRV are calculated by our program. Four-Hertz sampling rate is used to resample the 5-min RR interval for the ApEn calculation. Natural logarithm is taken of the RR interval before the calculation of fractal dimension. The nonlinear parameters are saved in the order of ApEn, Poincare, and FD. 


\section{HRV Plot}

When you select the submenu 'Plot HRV for Each Infant" under 'Analysis' of the main menu bar, the following window will pop up.

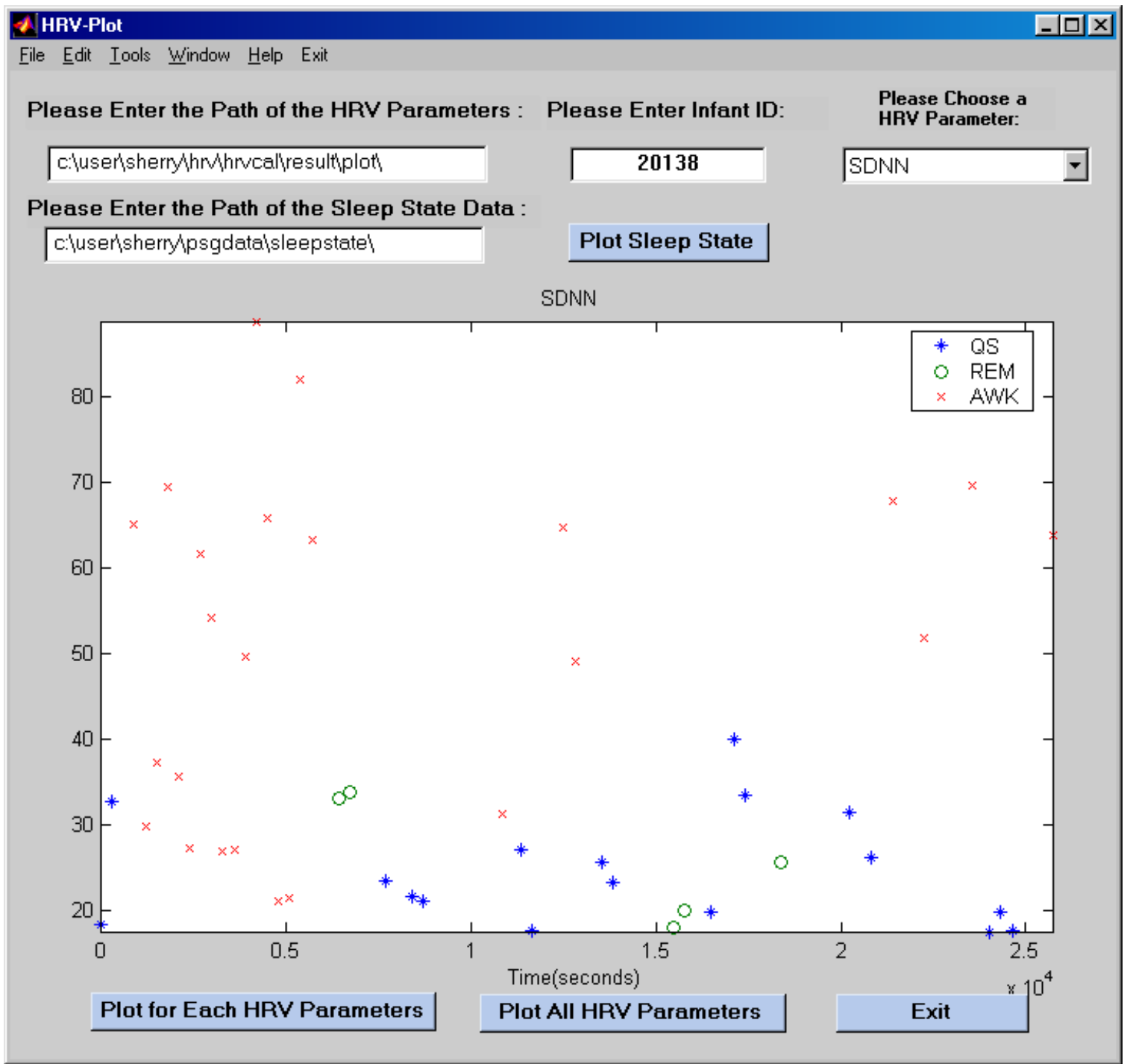

This interface allows the user to plot the calculated HRV parameters versus time for each infant. When given the path of the HRV parameters and sleep state files, the infant ID, and a specific parameter that you want to plot, that HRV values will show up 
in the plotting area by clicking the button " Plot for Each HRV Parameters". Each point in the plot indicates the HRV value for a 5-min segment in a specific sleep state. The sleep state of the chosen infant can be plotted in the plotting area by clicking the button "Plot sleep state". User can also choose to plot all time-domain, frequency-domain, and nonlinear measurements of HRV by clicking the "Plot All HRV Parameters" button, which will plot three to four HRV parameters and sleep state of each infant in one figure. The following figure shows an example.
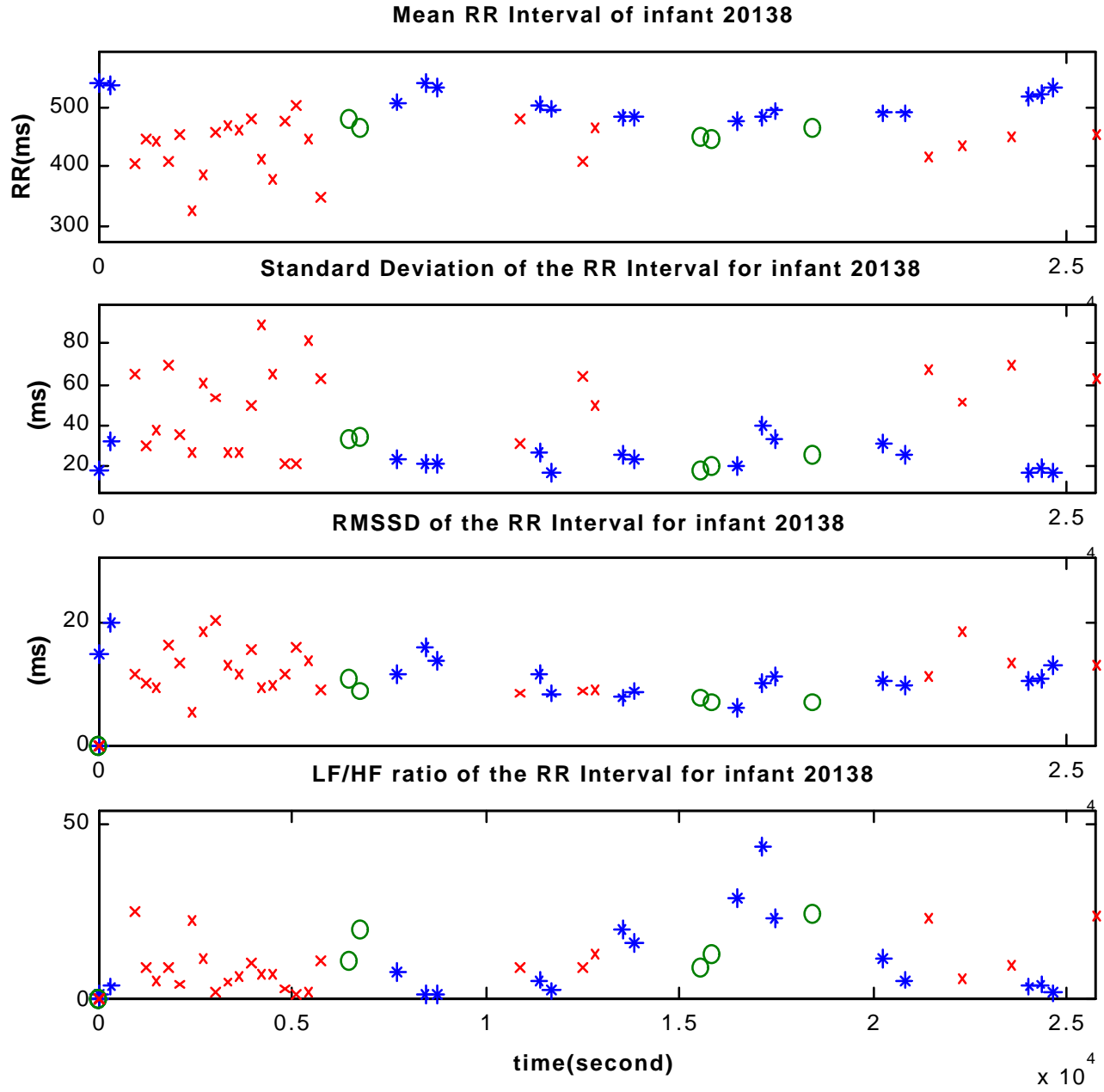


\section{Link HRV parameters of all infants into one matrix}

When you select the submenu 'Generate whole HRV dataset' under 'Analysis' of the main menu bar, the following window will pop up.

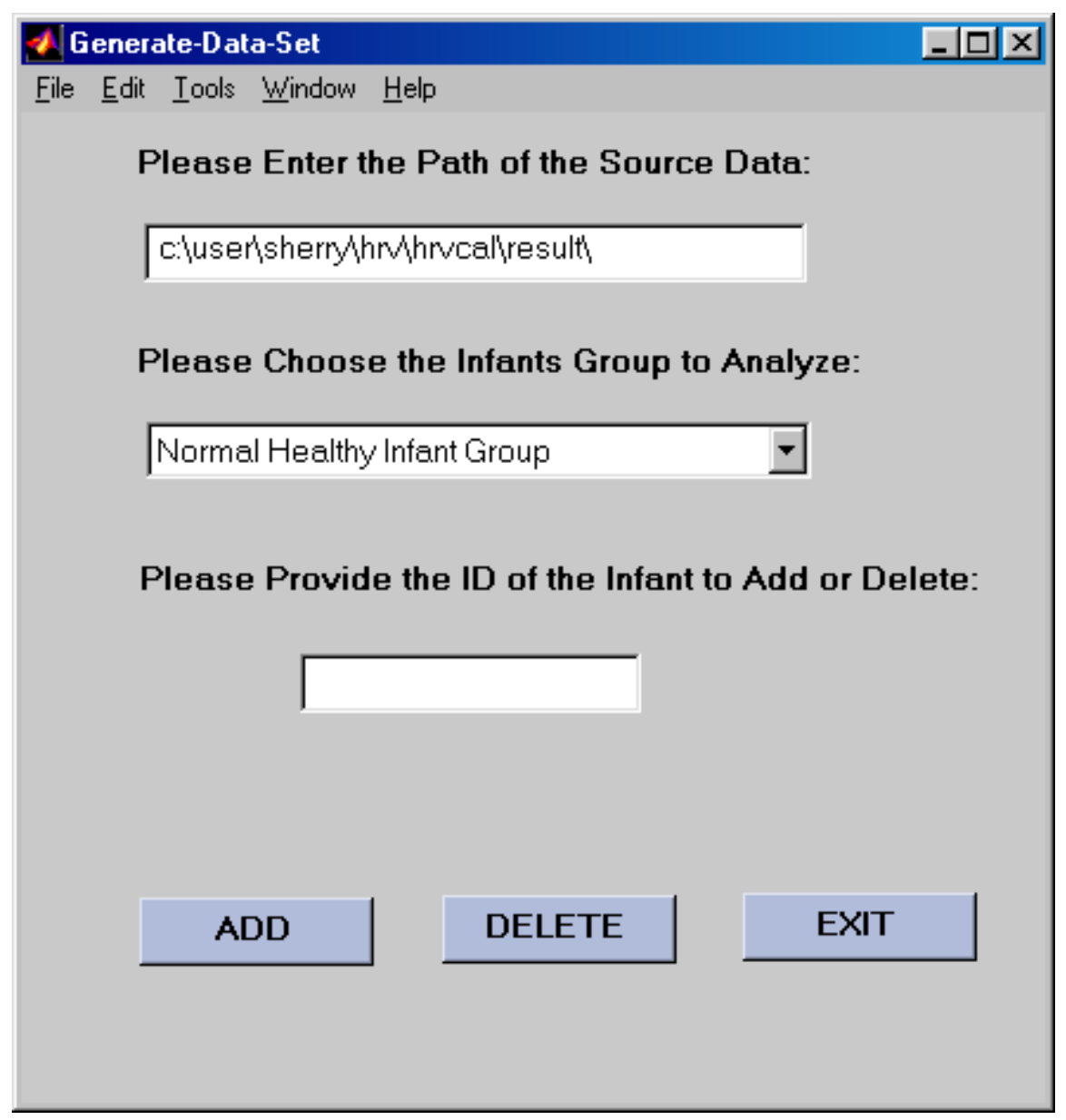

The interface "Generate-Whole-HRV-Dataset" fulfills the function of linking all the HRV parameters of selected infants into one matrix. It gives the user one matrix called "HRVALL", which is also saved in a file called "HRVALL". There are 28 columns in the matrix, which list HRV parameters, sleep state, segment number, infant name, and group information of all 5-min segments for the selected infants. The columns 
are in the order Mean, Median, SDNN, IQRNN, NIQRNN, CV, SDSD, IQRSD, NIQRSD, RMSSD, CVS, pNN50, HF, LF, NHF, NLF, total power, LF/HF ratio, HFW, LFW, LFW/LFW ratio, ApEn, Poincare, FD, sleep state code (0: QS, 1:REM, 2:AWK), segment number within each sleep state, infant ID, and group ID (0: healthy infants, 1 : infants with apparent life-threatening event). Each line is a record for one 5-min segment.

To use the interface, the path to the calculated HRV parameters, the group name, and the ID of the infant must be provided first. The file named "HRVALL" is generated to save the linked results, which is empty at the beginning. The file can be used to continue adding HRV values for the selected infant when clicking the "ADD" button. It contains the resultant HRV parameters for all three sleep states, It also has the function of deleting the record of a specific infant from the overall result matrix, which can be done by clicking the "DELETE" button. It will search for the record for that infant and delete them from the results matrix.

Both the add and delete procedure can be done for infants from a specific infant group by selecting the proper group name, which also means that it allows user to link the results of all the infants in one group, such as healthy, ALTE, SDS siblings, and preterm infant group. There is also one other selection under group name as "All Infants", which fulfils the function of linking all the infants' HRV results under a given directory. 


\section{Correlation of HRV Parameters}

When you select the submenu 'Correlation Between HRVs' under 'Analysis' of the main menu bar, the following window will pop up.

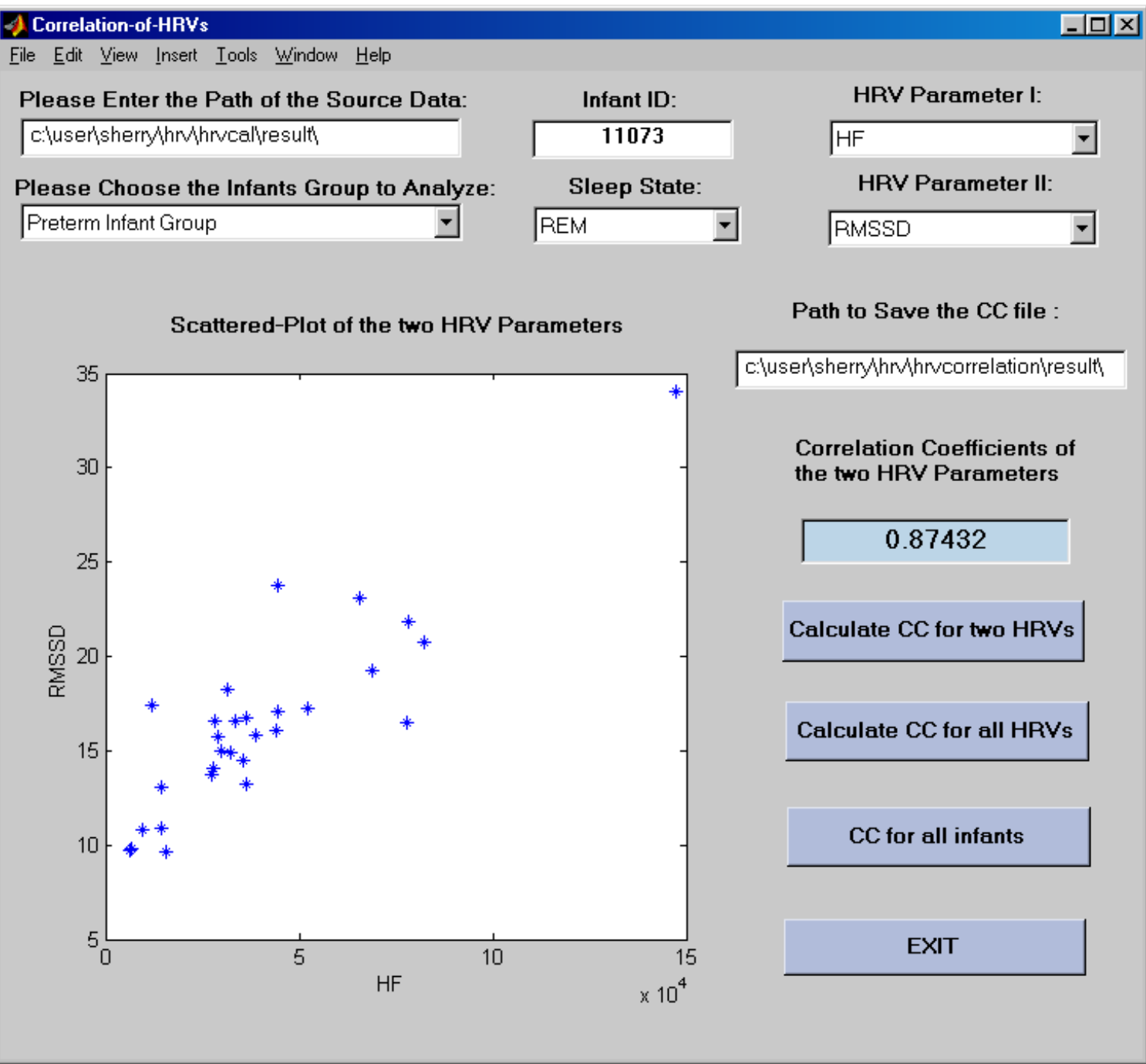

With this interface, the user can view the scatter plot and calculate the Pearson's correlation coefficient of any two selected HRV parameters for each infant with sleep state a consideration. First, the user needs to provide the path to the HRV parameters, group name, infant ID, and choose sleep state, and two HRV parameters from the popup 
menu. Then, the next step is the calculation of correlation coefficients. By click the 'Calculate CC for two HRVs' button, the scatter plot will be shown in the left plotting area, and the correlation coefficient of these two HRVs is shown in the edit window. The 'Calculate CC for all HRVs' fulfills the function of computing the correlation coefficients of all pairs of HRV parameters, for a selected sleep state. If any of QS, REM, or AWK is chosen, the resulted coefficients for this sleep state is saved in a variable named 'CCALL'. If 'ALL' instead of a specific sleep state is chosen, the correlations are calculated for all three sleep states, and saved in a file named '11073CCALLSS.mat' in the given directory. In that file, the resulted coefficients are in the order:

$\begin{array}{llll}\text { Mean-Median } & \text { Mean-SDNN } & \text { Mean-IQRNN .... } & \text { Median-SDNN..... } \\ \text { QS REM AWK } & \text { QS REM AWK } & \text { QS REM AWK } & \text { QS REM AWK }\end{array}$

The 'CC for all infants' button organizes the coefficients of a specific HRV parameters, which is indicated by 'HRV parameter I', with all other HRVs for all infants and sleep states. Also, the five point statistical characteristics $(2.5 \%, 25 \%, 50 \%, 75 \%$, and 97.5\%) and standard deviation of each pair of parameters per sleep state are calculated. The resultant matrix is saved in a file like 'Mean.mat'. 
APPENDIX B

RESULT OF DETECTING FAKE ARTIFACTS IN THE INFANT RR INTERVAL DATA 

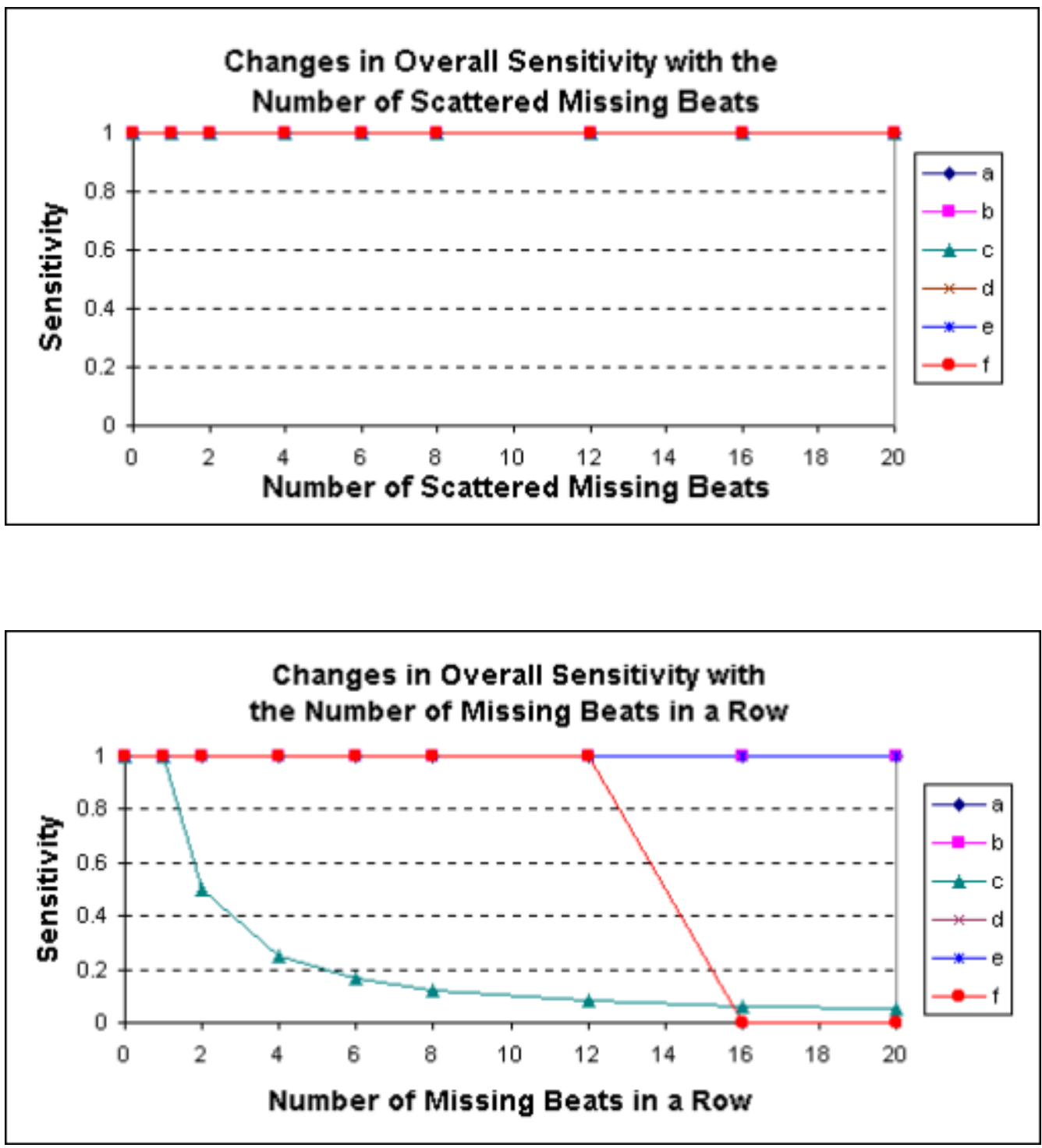

Figure B1 Changes of sensitivity of various algorithms with different amounts of artificial missing beats 

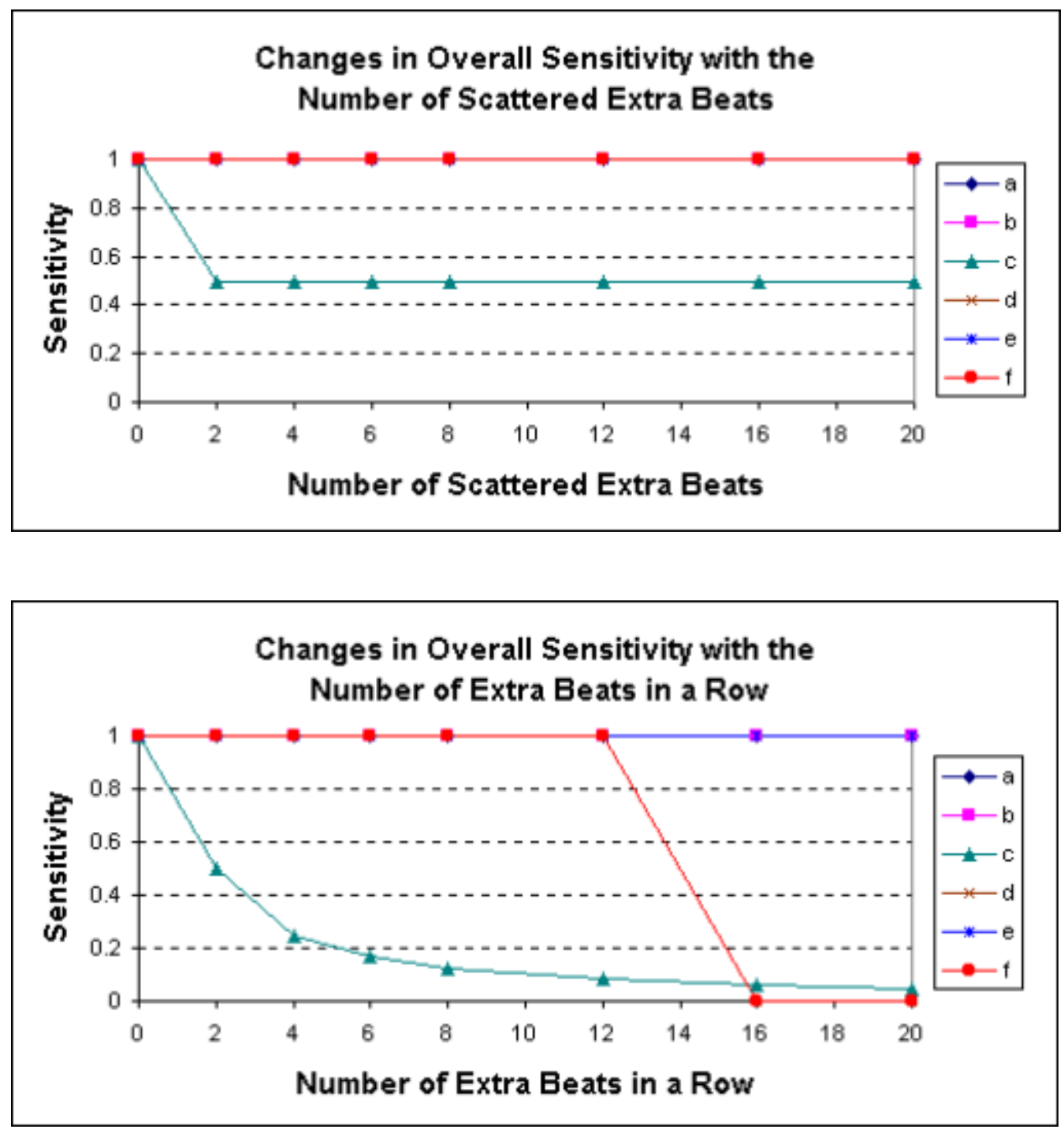

Figure B2 Changes of sensitivity of various algorithms with different amounts of artificial extra beats 

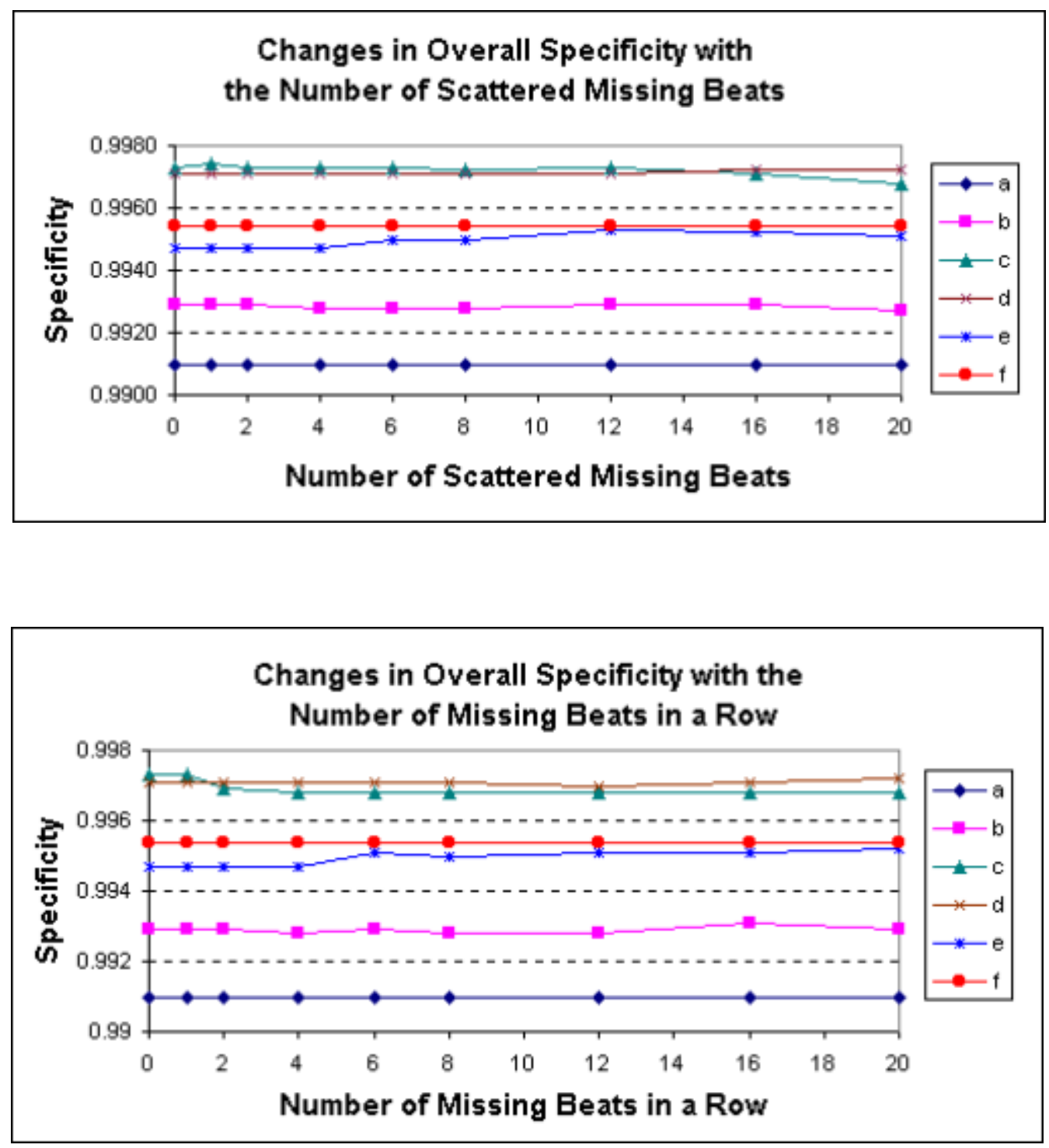

Figure B3 Changes of specificity of various algorithms with different amounts of artificial missing beats 

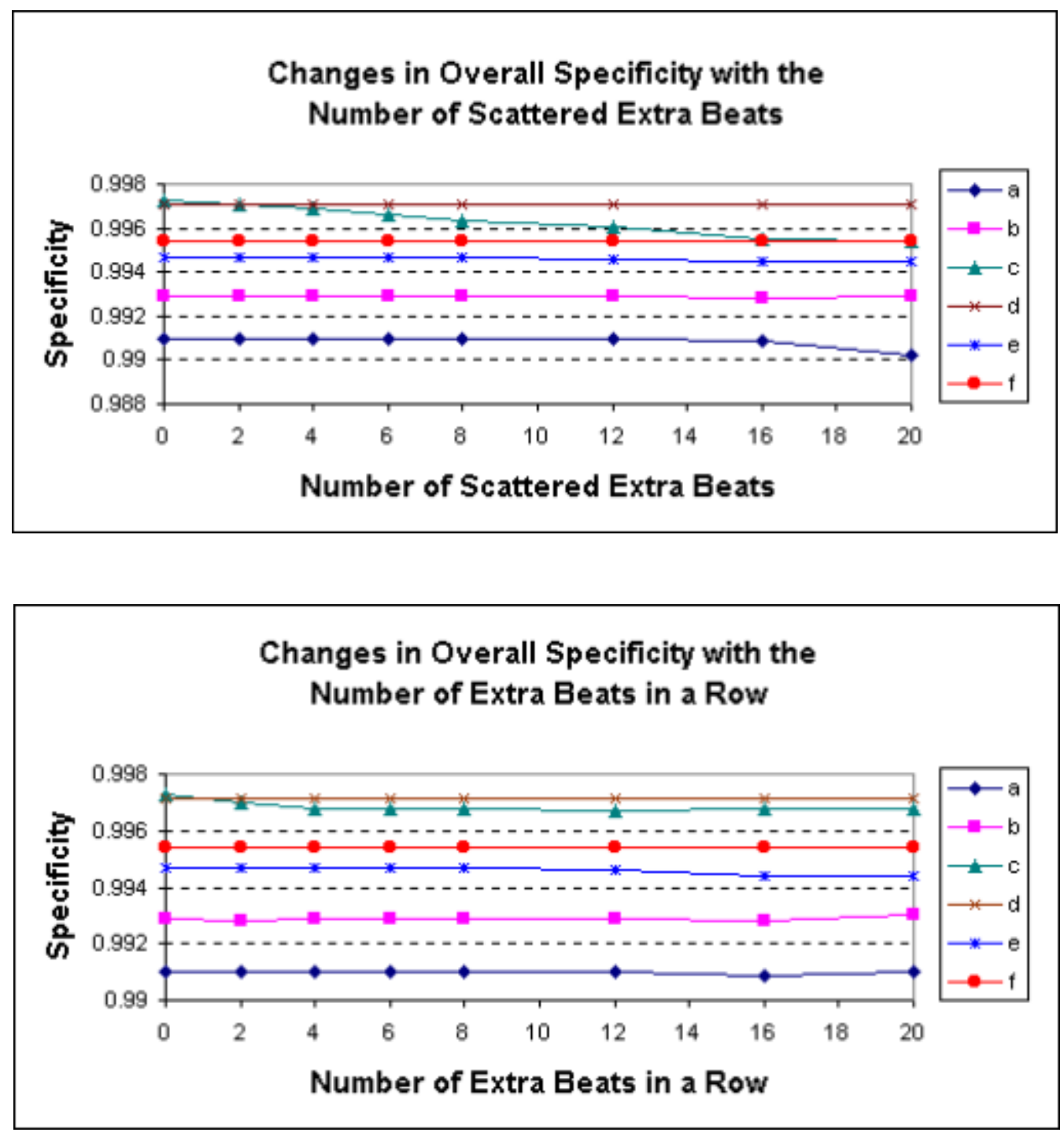

Figure B4 Changes of specificity of various algorithms with different amounts of artificial extra beats 


\section{APPENDIX C}

RESULT FOR THE EFFECT OF ARTIFACTS ON HRV PARAMETERS 
Table C1 HRV changes in percentage with two scattered missing beats

\begin{tabular}{|c|cc|cc|cc|cc|cc|}
\hline & \multicolumn{2}{|c}{ Mean } & \multicolumn{2}{c|}{ Median } & \multicolumn{2}{c|}{ SDNN } & \multicolumn{2}{c|}{ IQRNN } & \multicolumn{2}{c|}{ CV } \\
& noisy & $\mathrm{f}$ & noisy & $\mathrm{f}$ & noisy & $\mathrm{f}$ & noisy & $\mathrm{f}$ & noisy & $\mathrm{f}$ \\
\hline 12042 & 0.2920 & 0.0103 & 0.0000 & 0.0000 & 37.0064 & 0.0804 & 2.7778 & 0.6944 & 36.6076 & 0.0907 \\
12043 & 0.3231 & 0.0007 & 0.0000 & 0.0000 & 292.7941 & 0.1804 & 0.0000 & 0.0000 & 291.5291 & 0.1796 \\
12064 & 0.3185 & 0.0042 & 0.0000 & 0.0000 & 90.5753 & 0.0547 & 5.2632 & 1.3158 & 89.9703 & 0.0505 \\
23020 & 0.3030 & 0.4314 & 0.0000 & 0.0000 & 17.9573 & 9.0216 & 1.1628 & 2.3256 & 17.6009 & 8.6274 \\
31039 & 0.3240 & 0.1044 & 0.0000 & 0.0000 & 28.4800 & 3.8361 & 0.0000 & 0.0000 & 28.0651 & 3.7356 \\
41070 & 0.3225 & 0.0970 & 0.0000 & 0.1048 & 33.3242 & 2.0271 & 0.0000 & 0.0000 & 32.8956 & 1.9319 \\
50070 & 0.2270 & 0.0114 & 0.0000 & 0.0000 & 44.7604 & 0.0465 & 0.0000 & 0.0000 & 44.4325 & 0.0579 \\
50077 & 0.3241 & 0.1590 & 0.0000 & 0.0000 & 24.3435 & 7.5158 & 1.1628 & 1.7442 & 23.9417 & 7.3685 \\
50098 & 0.3258 & 0.0044 & 0.2012 & 0.0000 & 165.3510 & 0.0521 & 0.0000 & 0.0000 & 164.4894 & 0.0566 \\
20139 & 0.3536 & 1.2130 & 0.0000 & 0.1873 & 15.1193 & 17.4610 & 0.3436 & 7.5601 & 14.7137 & 16.4475 \\
\hline Mean & 0.3114 & 0.2036 & 0.0201 & 0.0292 & 74.9711 & 4.0276 & 1.0710 & 1.3640 & 74.4246 & 3.8546 \\
STD & 0.033641 & 0.3784 & 0.06363 & 0.0646 & 89.13458 & 5.76793 & 1.724606 & 2.3384 & 88.84072 & 5.46099 \\
\hline
\end{tabular}

\begin{tabular}{|c|cc|cc|cc|cc|cc|}
\hline & \multicolumn{2}{|c|}{ SDSD } & \multicolumn{2}{c|}{ IQRSD } & \multicolumn{2}{c|}{ RMSSD } & \multicolumn{2}{c|}{ ApEn } & \multicolumn{2}{c|}{ HF } \\
& noisy & $\mathrm{f}$ & noisy & $\mathrm{f}$ & noisy & $\mathrm{f}$ & noisy & $\mathrm{f}$ & noisy & $\mathrm{f}$ \\
\hline 12042 & 414.6935 & 0.1892 & 0.0000 & 0.0000 & 264.1420 & 0.1388 & 22.7239 & 0.2801 & 350.0235 & 29.3248 \\
12043 & 753.2995 & 0.2132 & 0.0000 & 0.0000 & 389.9423 & 0.1767 & 62.5174 & 1.1075 & 62.6037 & 0.3575 \\
12064 & 329.2537 & 0.0481 & 0.0000 & 0.0000 & 195.6300 & 0.0428 & 33.8482 & 1.0099 & 200.5043 & 59.8716 \\
23020 & 226.8892 & 17.8431 & 0.0000 & 0.0000 & 163.5755 & 12.4294 & 12.0159 & 8.3850 & 44.2324 & 1.7159 \\
31039 & 129.7228 & 11.9558 & 0.0000 & 5.5556 & 86.9784 & 8.8877 & 16.2428 & 1.8838 & 197.2297 & 9.6722 \\
41070 & 182.0234 & 14.3807 & 0.0000 & 0.0000 & 119.3410 & 9.1568 & 22.2126 & 0.2663 & 197.5209 & 13.8425 \\
50070 & 122.8145 & 0.0883 & 11.1111 & 0.0000 & 88.7808 & 0.0249 & 9.9530 & 1.2804 & 106.2375 & 0.1092 \\
50077 & 130.9119 & 28.2432 & 6.6667 & 6.6667 & 83.9363 & 16.7277 & 13.5126 & 4.7745 & 168.9639 & 0.7706 \\
50098 & 469.3544 & 0.4625 & 0.0000 & 0.0000 & 237.4551 & 0.3092 & 44.8326 & 0.2353 & 413.8512 & 0.7263 \\
20139 & 48.6771 & 41.4456 & 0.0000 & 15.7895 & 33.1468 & 30.5380 & 9.5270 & 12.3436 & 70.0004 & 72.7593 \\
\hline Mean & 280.7640 & 11.4870 & 1.7778 & 2.8012 & 166.2928 & 7.8432 & 24.7386 & 3.1566 & 181.1167 & 18.9150 \\
STD & 214.9621 & 14.393 & 3.89153 & 5.2297 & 107.7752 & 10.0814 & 17.43083 & 4.1294 & 122.24 & 26.7708 \\
\hline
\end{tabular}

\begin{tabular}{|c|cc|cc|cc|cc|cc|}
\hline & \multicolumn{2}{|c|}{ NHF } & \multicolumn{2}{c|}{ LF } & \multicolumn{2}{c|}{ NLF } & \multicolumn{2}{c|}{ Total Power } & \multicolumn{2}{c|}{ LF/HF } \\
& noisy & $\mathrm{f}$ & noisy & $\mathrm{f}$ & noisy & $\mathrm{f}$ & noisy & $\mathrm{f}$ & noisy & $\mathrm{f}$ \\
\hline 12042 & 201.0367 & 28.6223 & 19.6005 & 1.8344 & 19.9949 & 2.8467 & 49.4912 & 0.9843 & 73.4235 & 44.0880 \\
12043 & 0.3037 & 0.0200 & 63.9965 & 0.4135 & 0.5502 & 0.0362 & 63.0990 & 0.3774 & 0.8565 & 0.0562 \\
12064 & 6.4599 & 1.3686 & 270.2655 & 52.6160 & 15.2551 & 3.2319 & 221.2573 & 57.7132 & 23.2147 & 4.5384 \\
23020 & 12.8756 & 1.2046 & 66.7532 & 2.9519 & 0.7282 & 0.0681 & 65.5477 & 2.8858 & 15.6143 & 1.2576 \\
31039 & 43.3807 & 4.2257 & 63.8344 & 15.1046 & 20.9679 & 2.0425 & 107.3011 & 13.3345 & 44.8795 & 6.0140 \\
41070 & 90.4900 & 71.6692 & 26.8756 & 57.2717 & 18.7670 & 14.8637 & 56.1872 & 49.8119 & 57.3558 & 50.4068 \\
50070 & 12.8970 & 0.1942 & 56.6188 & 0.2995 & 14.2650 & 0.2148 & 82.6777 & 0.0848 & 24.0590 & 0.4083 \\
50077 & 34.7758 & 0.2165 & 65.6503 & 0.4502 & 16.9939 & 0.1058 & 99.5639 & 0.5554 & 38.4117 & 0.3230 \\
50098 & 48.1203 & 0.5557 & 221.2047 & 0.3110 & 30.6946 & 0.3544 & 246.9148 & 0.1716 & 53.2101 & 0.9152 \\
20139 & 21.1438 & 23.7570 & 29.4352 & 61.1546 & 7.7633 & 8.7227 & 40.3294 & 64.2712 & 23.8618 & 42.6003 \\
\hline Mean & 47.1483 & 13.1834 & 88.4235 & 19.2407 & 14.5980 & 3.2487 & 103.2369 & 19.0190 & 35.4887 & 15.0608 \\
STD & 60.18704 & 23.103 & 85.5718 & 26.514 & 9.362397 & 4.88063 & 72.3541 & 26.9014 & 21.9566 & 21.3192 \\
\hline
\end{tabular}


Table $\mathrm{C} 2 \mathrm{HRV}$ changes in percentage due to false positive of algorithm $\mathrm{f}$

\begin{tabular}{|c|cc|cc|cc|cc|cc|}
\hline & \multicolumn{2}{|c|}{ Mean } & \multicolumn{2}{c|}{ Median } & \multicolumn{2}{c|}{ SDNN } & \multicolumn{2}{c|}{ IQRNN } & \multicolumn{2}{c|}{ CV } \\
& noisy & $\mathrm{f}$ & noisy & $\mathrm{f}$ & noisy & $\mathrm{f}$ & noisy & $\mathrm{f}$ & noisy & $\mathrm{f}$ \\
\hline 12042 & 0.0000 & 0.0000 & 0.0000 & 0.0000 & 0.0000 & 0.0000 & 0.0000 & 0.0000 & 0.0000 & 0.0000 \\
12043 & 0.0000 & 0.0000 & 0.0000 & 0.0000 & 0.0000 & 0.0000 & 0.0000 & 0.0000 & 0.0000 & 0.0000 \\
12064 & 0.0000 & 0.0000 & 0.0000 & 0.0000 & 0.0000 & 0.0000 & 0.0000 & 0.0000 & 0.0000 & 0.0000 \\
23020 & 0.0000 & 0.4100 & 0.0000 & 0.0000 & 0.0000 & 9.0567 & 0.0000 & 2.3256 & 0.0000 & 8.6822 \\
31039 & 0.0000 & 0.1009 & 0.0000 & 0.0000 & 0.0000 & 3.8812 & 0.0000 & 0.0000 & 0.0000 & 3.7841 \\
41070 & 0.0000 & 0.0733 & 0.0000 & 0.0000 & 0.0000 & 2.0119 & 0.0000 & 1.1364 & 0.0000 & 1.9400 \\
50070 & 0.0000 & 0.0000 & 0.0000 & 0.0000 & 0.0000 & 0.0000 & 0.0000 & 0.0000 & 0.0000 & 0.0000 \\
50077 & 0.0000 & 0.1662 & 0.0000 & 0.0000 & 0.0000 & 7.6316 & 0.0000 & 1.1628 & 0.0000 & 7.4778 \\
50098 & 0.0000 & 0.0000 & 0.0000 & 0.0000 & 0.0000 & 0.0000 & 0.0000 & 0.0000 & 0.0000 & 0.0000 \\
20139 & 0.0000 & 1.1064 & 0.0000 & 0.1873 & 0.0000 & 16.8337 & 0.0000 & 6.8729 & 0.0000 & 15.9032 \\
\hline Mean & 0.0000 & 0.1857 & 0.0000 & 0.0187 & 0.0000 & 3.9415 & 0.0000 & 1.1498 & 0.0000 & 3.7787 \\
STD & 0 & 0.34812 & 0 & 0.05923 & 0 & 5.6509 & 0 & 2.162 & 0 & 5.36973 \\
\hline
\end{tabular}

\begin{tabular}{|c|cc|cc|cc|cc|cc|}
\hline & \multicolumn{2}{|c|}{ SDSD } & \multicolumn{2}{c|}{ IQRSD } & \multicolumn{2}{c|}{ RMSSD } & \multicolumn{2}{c|}{ ApEn } & \multicolumn{2}{c|}{ HF } \\
& noisy & $\mathrm{f}$ & noisy & $\mathrm{f}$ & noisy & $\mathrm{f}$ & noisy & $\mathrm{f}$ & noisy & $\mathrm{f}$ \\
\hline 12042 & 0.0000 & 0.0000 & 0.0000 & 0.0000 & 0.0000 & 0.0000 & 0.0000 & 0.0000 & 0.0000 & 0.0000 \\
12043 & 0.0000 & 0.0000 & 0.0000 & 0.0000 & 0.0000 & 0.0000 & 0.0000 & 0.0000 & 0.0000 & 0.0000 \\
12064 & 0.0000 & 0.0000 & 0.0000 & 0.0000 & 0.0000 & 0.0000 & 0.0000 & 0.0000 & 0.0000 & 0.0000 \\
23020 & 0.0000 & 18.1184 & 0.0000 & 0.0000 & 0.0000 & 12.7089 & 0.0000 & 7.3460 & 0.0000 & 43.7321 \\
31039 & 0.0000 & 11.8368 & 0.0000 & 5.5556 & 0.0000 & 8.6883 & 0.0000 & 1.3663 & 0.0000 & 7.5611 \\
41070 & 0.0000 & 14.4483 & 0.0000 & 0.0000 & 0.0000 & 9.1358 & 0.0000 & 0.8146 & 0.0000 & 2.0230 \\
50070 & 0.0000 & 0.0000 & 0.0000 & 0.0000 & 0.0000 & 0.0000 & 0.0000 & 0.0000 & 0.0000 & 0.0000 \\
50077 & 0.0000 & 28.2968 & 0.0000 & 0.0000 & 0.0000 & 16.7016 & 0.0000 & 5.0604 & 0.0000 & 0.0000 \\
50098 & 0.0000 & 0.0000 & 0.0000 & 0.0000 & 0.0000 & 0.0000 & 0.0000 & 0.0000 & 0.0000 & 0.0000 \\
20139 & 0.0000 & 40.8940 & 0.0000 & 15.7895 & 0.0000 & 29.7226 & 0.0000 & 10.3531 & 0.0000 & 71.9833 \\
\hline Mean & 0.0000 & 11.3594 & 0.0000 & 2.1345 & 0.0000 & 7.6957 & 0.0000 & 2.4940 & 0.0000 & 12.5300 \\
STD & 0 & 14.3752 & 0 & 5.10568 & 0 & 9.94765 & 0 & 3.75774 & 0 & 24.91175 \\
\hline
\end{tabular}

\begin{tabular}{|c|cc|cc|cc|cc|cc|}
\hline Missed & \multicolumn{2}{|c|}{ NHF } & \multicolumn{2}{c|}{ LF } & \multicolumn{2}{c|}{ NLF } & \multicolumn{2}{c|}{ Total Power } & \multicolumn{2}{c|}{ LF/HF } \\
Beats & noisy & $\mathrm{f}$ & noisy & $\mathrm{f}$ & noisy & $\mathrm{f}$ & noisy & $\mathrm{f}$ & noisy & $\mathrm{f}$ \\
\hline 12042 & 0.0000 & 0.0000 & 0.0000 & 0.0000 & 0.0000 & 0.0000 & 0.0000 & 0.0000 & 0.0000 & 0.0000 \\
12043 & 0.0000 & 0.0000 & 0.0000 & 0.0000 & 0.0000 & 0.0000 & 0.0000 & 0.0000 & 0.0000 & 0.0000 \\
12064 & 0.0000 & 0.0000 & 0.0000 & 0.0000 & 0.0000 & 0.0000 & 0.0000 & 0.0000 & 0.0000 & 0.0000 \\
23020 & 0.0000 & 11.0095 & 0.0000 & 62.5198 & 0.0000 & 0.6227 & 0.0000 & 61.5141 & 0.0000 & 13.0713 \\
31039 & 0.0000 & 4.6709 & 0.0000 & 0.8425 & 0.0000 & 2.2577 & 0.0000 & 3.0317 & 0.0000 & 7.2681 \\
41070 & 0.0000 & 1.8927 & 0.0000 & 0.2592 & 0.0000 & 0.3925 & 0.0000 & 0.1328 & 0.0000 & 2.3294 \\
50070 & 0.0000 & 0.0000 & 0.0000 & 0.0000 & 0.0000 & 0.0000 & 0.0000 & 0.0000 & 0.0000 & 0.0000 \\
50077 & 0.0000 & 0.0000 & 0.0000 & 0.0000 & 0.0000 & 0.0000 & 0.0000 & 0.0000 & 0.0000 & 0.0000 \\
50098 & 0.0000 & 0.0000 & 0.0000 & 0.0000 & 0.0000 & 0.0000 & 0.0000 & 0.0000 & 0.0000 & 0.0000 \\
20139 & 0.0000 & 52.2705 & 0.0000 & 30.0356 & 0.0000 & 19.1919 & 0.0000 & 41.3011 & 0.0000 & 149.7236 \\
\hline Mean & 0.0000 & 6.9844 & 0.0000 & 9.3657 & 0.0000 & 2.2465 & 0.0000 & 10.5980 & 0.0000 & 17.2392 \\
STD & 0 & 16.2993 & 0 & 20.9085 & 0 & 5.99538 & 0.0000 & 22.0500 & 0 & 46.7551 \\
\hline
\end{tabular}


Table C3 Averaged HRV value for each 15-min segment of each infants

MEAN

\begin{tabular}{|c|c|c|c|c|c|c|}
\hline & \multicolumn{2}{|c|}{ Artifact -Free } & \multicolumn{2}{|c|}{ With type $4 \& 5$ Artifacts } & \multicolumn{2}{|c|}{ Cleaned by Algorithm f } \\
\hline & $\operatorname{seg} 1$ & seg2 & $\operatorname{seg} 1$ & seg2 & $\operatorname{seg} 1$ & seg2 \\
\hline 12042 & 462.6010 & 344.7708 & 0.0000 & 0.5108 & 0.0000 & 0.8579 \\
\hline 12043 & 492.4321 & 393.3018 & 0.0000 & 0.0362 & 0.1212 & 0.0216 \\
\hline 12064 & 460.0508 & 437.4800 & 0.0000 & 0.0000 & 0.1084 & 0.3676 \\
\hline 23020 & 446.8800 & 385.2697 & 0.0000 & 1.5968 & 0.2507 & 0.2611 \\
\hline 31039 & 453.4727 & 423.2393 & 0.0000 & 0.0535 & 0.0425 & 0.0689 \\
\hline 41070 & 480.2781 & 464.8481 & 0.0000 & 1.1183 & 0.0242 & 1.2293 \\
\hline 50070 & 347.0854 & 422.8936 & 0.0000 & 0.0000 & 0.0039 & 0.7868 \\
\hline 50077 & 477.2369 & 581.4026 & 0.0000 & 0.0000 & 0.0720 & 0.9546 \\
\hline 50098 & 502.0102 & 397.8425 & 0.0000 & 0.0000 & 0.0000 & 0.0000 \\
\hline 20139 & 560.8690 & 593.8941 & 0.0000 & 0.0000 & 0.6963 & 1.5969 \\
\hline Mean & 468.2916 & 444.4942 & 0.0000 & 0.3316 & 0.1319 & 0.6145 \\
\hline STD & 53.73108349 & 82.12146449 & 0 & 0.574083438 & 0.212838932 & 0.553802479 \\
\hline
\end{tabular}

$\begin{array}{llll}\text { Mean of } & \text { (seg1\&seg2) } & 0.3732\end{array}$

MEDIAN

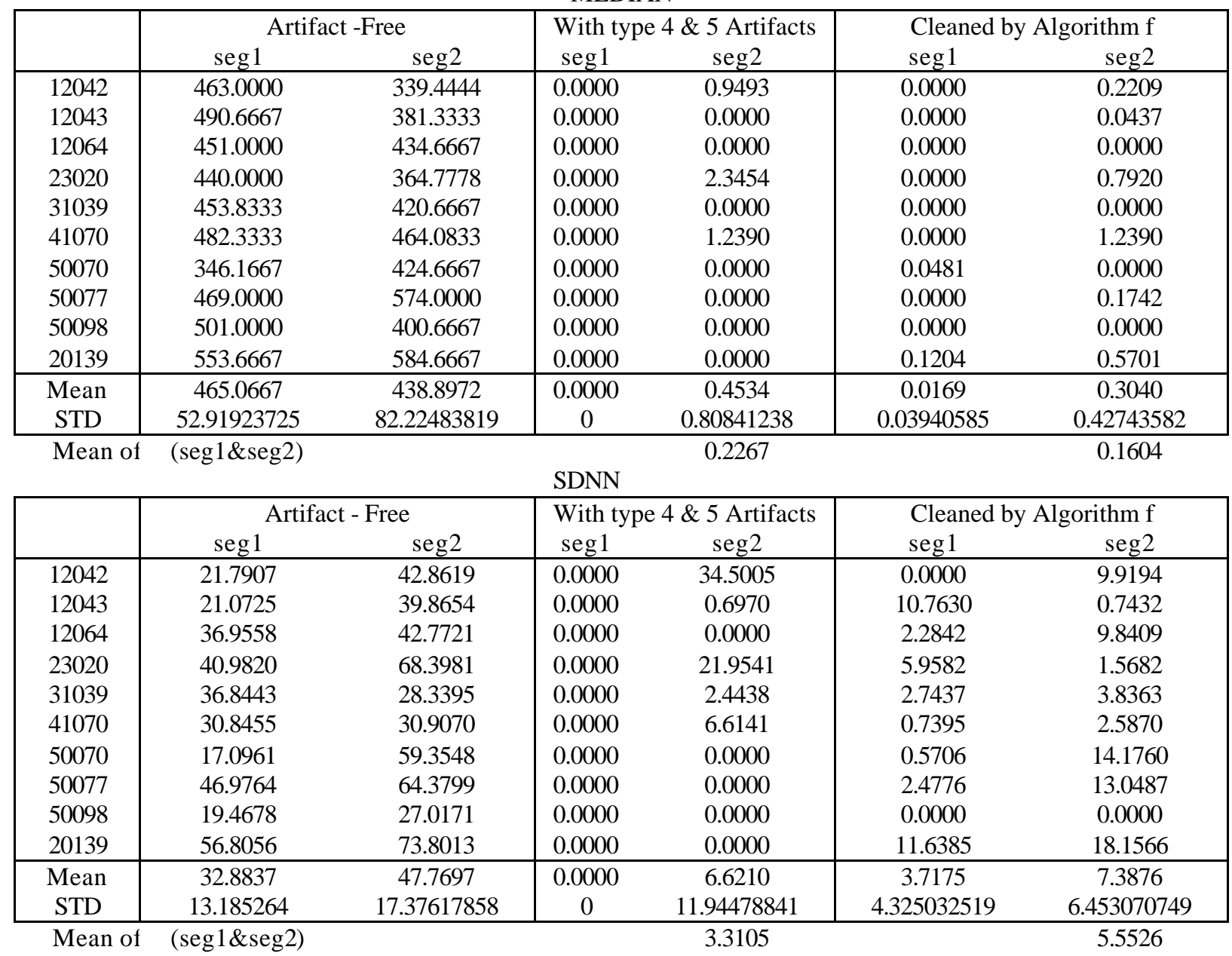


Table C3 Averaged HRV value for each 15-min segment of each infants (cont.)

IQRNN

\begin{tabular}{|c|cc|cc|cc|}
\hline & \multicolumn{2}{|c|}{ Artifact - Free } & \multicolumn{2}{c|}{ With type 4 \& 5 Artifacts } & \multicolumn{2}{c|}{ Cleaned by Algorithm f } \\
& seg1 & seg2 & seg1 & seg2 & seg1 & seg2 \\
\hline 12042 & 25.2500 & 56.8194 & 0.0000 & 9.1176 & 0.0000 & 3.0625 \\
12043 & 16.6667 & 53.5000 & 0.0000 & 0.3115 & 0.0000 & 0.3115 \\
12064 & 52.3333 & 41.7500 & 0.0000 & 0.0000 & 0.9554 & 0.0000 \\
23020 & 39.3333 & 96.6538 & 0.0000 & 3.6488 & 1.6949 & 0.5306 \\
31039 & 48.3333 & 37.6667 & 0.0000 & 0.5900 & 0.0000 & 0.8850 \\
41070 & 39.8333 & 39.2708 & 0.0000 & 0.4775 & 0.0000 & 0.0531 \\
50070 & 24.5000 & 79.4167 & 0.0000 & 0.0000 & 0.0000 & 0.7345 \\
50077 & 56.0833 & 73.5833 & 0.0000 & 0.0000 & 0.1486 & 8.4371 \\
50098 & 18.6667 & 30.6667 & 0.0000 & 0.0000 & 0.0000 & 0.0000 \\
20139 & 70.5833 & 93.2500 & 0.0000 & 0.0000 & 5.4309 & 9.0706 \\
\hline Mean & 39.1583 & 60.2577 & 0.0000 & 1.4145 & 0.8230 & 2.3085 \\
STD & 17.84602545 & 24.00061048 & 0 & 2.927040673 & 1.716897024 & 3.515882525 \\
\hline
\end{tabular}

Mean of (seg1\&seg2)

0.7073

1.5657

CV

\begin{tabular}{|c|cc|cc|cc|}
\hline & \multicolumn{2}{|c|}{ Artifact -Free } & \multicolumn{2}{c|}{ With type 4 \& 5 Artifacts } & \multicolumn{2}{c|}{ Cleaned by Algorithm f } \\
& seg1 & seg2 & seg1 & seg2 & seg1 & seg2 \\
\hline 12042 & 4.7458 & 12.6967 & 0.0000 & 35.6571 & 0.0000 & 11.1590 \\
12043 & 4.2092 & 10.3989 & 0.0000 & 0.7554 & 10.4320 & 0.7070 \\
12064 & 8.3771 & 9.7734 & 0.0000 & 0.0000 & 2.0032 & 9.5067 \\
23020 & 9.1698 & 18.1925 & 0.0000 & 20.6471 & 5.7036 & 1.1316 \\
31039 & 8.1423 & 6.7348 & 0.0000 & 2.6488 & 2.6836 & 3.5226 \\
41070 & 6.4472 & 6.6577 & 0.0000 & 7.6687 & 0.7188 & 1.4823 \\
50070 & 4.9300 & 14.0417 & 0.0000 & 0.0000 & 0.5927 & 13.2818 \\
50077 & 9.8591 & 11.0545 & 0.0000 & 0.0000 & 2.3917 & 12.1883 \\
50098 & 3.8553 & 6.9265 & 0.0000 & 0.0000 & 0.0000 & 0.0000 \\
20139 & 10.1580 & 12.4476 & 0.0000 & 0.0000 & 11.0777 & 16.5723 \\
\hline Mean & 6.9894 & 10.8924 & 0.0000 & 6.7377 & 3.5603 & 6.9552 \\
STD & 2.43595101 & 3.671988578 & 0 & 12.06538303 & 4.151516687 & 6.210631706 \\
\hline
\end{tabular}

Mean of (seg1\&seg2)

3.3689

5.2577

SDSD

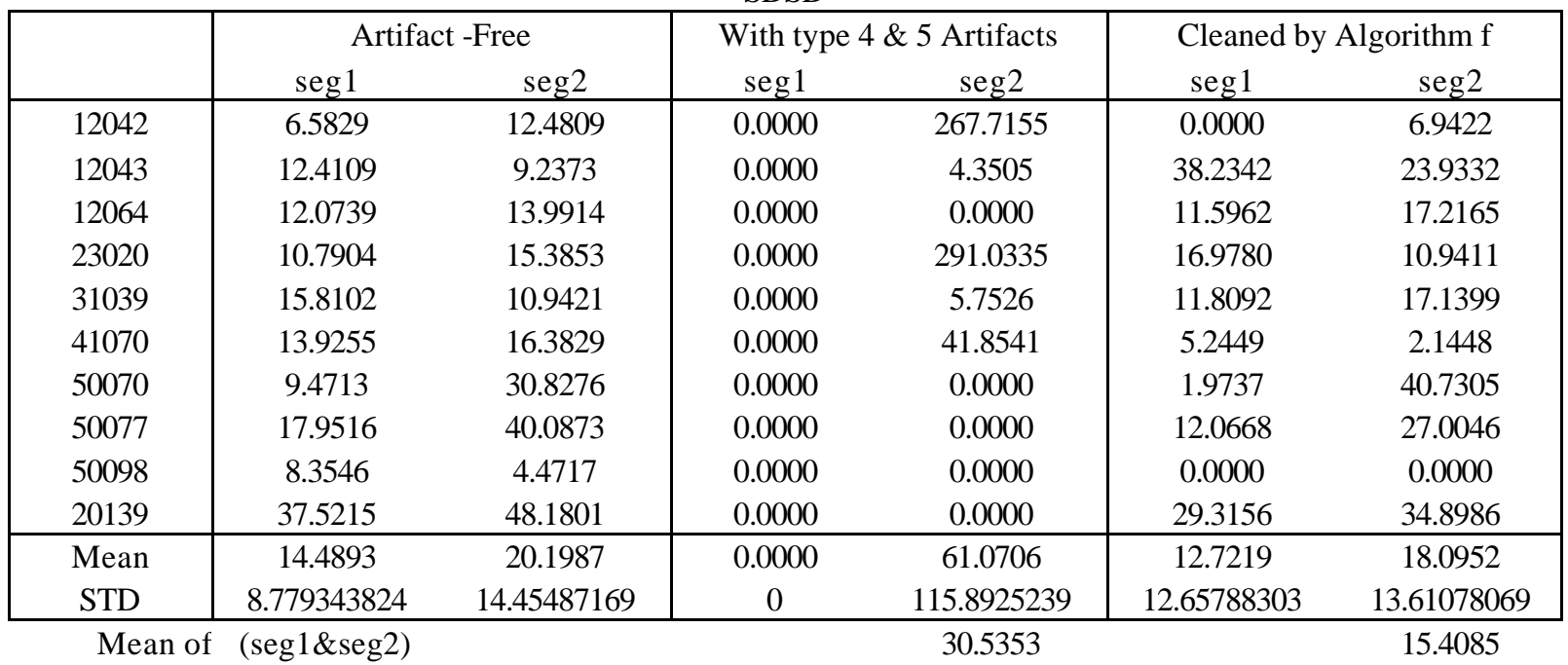


Table C3 Averaged HRV value for each 15-min segment of each infants (cont.)

IQRSD

\begin{tabular}{|c|cc|cc|cc|}
\hline & \multicolumn{2}{|c|}{ Artifact -Free } & \multicolumn{2}{c|}{ With type 4 \& 5 Artifacts } & \multicolumn{2}{c|}{ Cleaned by Algorithm f } \\
& seg1 & seg2 & seg1 & seg2 & seg1 & seg2 \\
\hline 12042 & 7.3333 & 7.3333 & 0.0000 & 169.6970 & 0.0000 & 40.9091 \\
12043 & 8.3333 & 5.6667 & 0.0000 & 0.0000 & 0.0000 & 0.0000 \\
12064 & 11.0000 & 10.3333 & 0.0000 & 0.0000 & 3.0303 & 3.4946 \\
23020 & 8.6667 & 8.0143 & 0.0000 & 226.5003 & 0.0000 & 33.0957 \\
31039 & 17.2222 & 10.6667 & 0.0000 & 0.0000 & 3.3871 & 0.0000 \\
41070 & 16.5833 & 17.6354 & 0.0000 & 2.0673 & 0.0000 & 3.0124 \\
50070 & 9.7500 & 25.7500 & 0.0000 & 0.0000 & 2.5641 & 5.5016 \\
50077 & 16.0000 & 43.8333 & 0.0000 & 0.0000 & 0.0000 & 13.0228 \\
50098 & 9.3333 & 4.0000 & 0.0000 & 0.0000 & 0.0000 & 0.0000 \\
20139 & 38.6667 & 48.6667 & 0.0000 & 0.0000 & 8.6207 & 17.0091 \\
\hline Mean & 14.2889 & 18.1900 & 0.0000 & 39.8265 & 1.7602 & 11.6045 \\
STD & 9.324439189 & 16.13071269 & 0 & 84.48685089 & 2.800046773 & 14.65409855 \\
\hline
\end{tabular}

Mean of (seg1\&seg2)

19.9132 6.6824

RMSSD

\begin{tabular}{|c|cc|cc|cc|}
\hline & \multicolumn{2}{|c|}{ Artifact -Free } & \multicolumn{2}{c|}{ With type 4 \& 5 Artifacts } & \multicolumn{2}{c|}{ Cleaned by Algorithm f } \\
& seg1 & seg2 & seg1 & seg2 & seg1 & seg2 \\
\hline 12042 & 10.0305 & 14.9887 & 0.0000 & 244.0322 & 0.0000 & 11.8775 \\
12043 & 15.9144 & 11.5107 & 0.0000 & 3.6883 & 27.4382 & 17.4485 \\
12064 & 16.3880 & 17.4960 & 0.0000 & 0.0000 & 7.3288 & 12.7542 \\
23020 & 14.2140 & 17.7908 & 0.0000 & 277.5895 & 11.6010 & 2.5695 \\
31039 & 22.3373 & 14.8333 & 0.0000 & 3.9856 & 7.1848 & 9.9376 \\
41070 & 20.9811 & 23.6879 & 0.0000 & 27.9790 & 3.0670 & 0.1961 \\
50070 & 12.6404 & 39.3836 & 0.0000 & 0.0000 & 0.7473 & 31.1324 \\
50077 & 24.0905 & 60.8582 & 0.0000 & 0.0000 & 7.6376 & 19.6888 \\
50098 & 13.1146 & 5.9250 & 0.0000 & 0.0000 & 0.0000 & 0.0000 \\
20139 & 55.9207 & 70.2174 & 0.0000 & 0.0000 & 18.9465 & 26.4040 \\
\hline Mean & 20.5632 & 27.6692 & 0.0000 & 55.7275 & 8.3951 & 13.2009 \\
STD & 13.22650038 & 21.92279388 & 0 & 108.7118826 & 8.91477343 & 10.67191443 \\
\hline
\end{tabular}

$\begin{array}{lll}\text { Mean of (seg1\&seg2) } & 27.8637 & 10.7980\end{array}$

ApEn

\begin{tabular}{|c|cc|cc|cc|}
\hline & \multicolumn{2}{|c|}{ Artifact - Free } & \multicolumn{2}{c|}{ With type 4 \& 5 Artifacts } & \multicolumn{2}{c|}{ Cleaned by Algorithm f } \\
& seg1 & seg2 & seg1 & seg2 & seg 2 \\
\hline 12042 & 0.9596 & 0.7059 & 0.0000 & 8.2284 & 0.0000 & 3.9437 \\
12043 & 1.1480 & 0.5742 & 0.0000 & 0.1602 & 2.6343 & 1.0058 \\
12064 & 0.8378 & 0.6339 & 0.0000 & 0.0000 & 1.2775 & 8.6099 \\
23020 & 0.6324 & 0.4391 & 0.0000 & 15.9199 & 3.1258 & 24.1589 \\
31039 & 1.0540 & 1.0205 & 0.0000 & 0.5263 & 1.7301 & 3.7504 \\
41070 & 1.0670 & 1.1366 & 0.0000 & 0.0977 & 0.2645 & 4.1201 \\
50070 & 1.1992 & 0.8445 & 0.0000 & 0.0000 & 0.1652 & 12.9165 \\
50077 & 0.7881 & 0.9436 & 0.0000 & 0.0000 & 2.3949 & 4.7455 \\
50098 & 1.1588 & 0.5746 & 0.0000 & 0.0000 & 0.0000 & 0.0000 \\
20139 & 0.9836 & 0.9205 & 0.0000 & 0.0000 & 6.8745 & 5.3653 \\
\hline Mean & 0.9828 & 0.7793 & 0.0000 & 2.4932 & 1.8467 & 6.8616 \\
STD & 0.182413368 & 0.226874499 & 0 & 5.367581947 & 2.11672209 & 7.092346119 \\
\hline
\end{tabular}

Mean of (seg1\&seg2) 1.2466 4.3541 
Table C3 Averaged HRV value for each 15-min segment of each infants (cont.)

$\mathrm{HF}$

\begin{tabular}{|c|cc|cc|cc|}
\hline & \multicolumn{2}{|c|}{ Artifact -Free } & \multicolumn{2}{c|}{ With type 4 \& 5 Artifacts } & \multicolumn{2}{c|}{ Cleaned by Algorithm f } \\
& $\mathrm{seg} 1$ & $\mathrm{seg} 2$ & $\mathrm{seg} 1$ & $\mathrm{seg} 2$ & $\mathrm{seg} 1$ & $\mathrm{seg} 2$ \\
\hline 12042 & 15534.5230 & 38958.0640 & 0.0000 & 3148.8664 & 0.0000 & 35.5328 \\
12043 & 8138.1936 & 16900.7830 & 0.0000 & 28.4906 & 0.0000 & 36.0507 \\
12064 & 34829.0110 & 53906.0690 & 0.0000 & 0.0000 & 11.0800 & 15.3355 \\
23020 & 20212.1020 & 35219.5990 & 0.0000 & 163.4165 & 17.9801 & 37.9650 \\
31039 & 71776.9960 & 21859.2230 & 0.0000 & 0.0875 & 24.4408 & 7.8247 \\
41070 & 40883.4740 & 63244.9360 & 0.0000 & 50.9213 & 0.6254 & 12.0111 \\
50070 & 17577.8190 & 284267.8000 & 0.0000 & 0.0000 & 3.3596 & 63.4952 \\
50077 & 92598.7720 & 543824.8500 & 0.0000 & 0.0000 & 0.0000 & 42.5651 \\
50098 & 37690.3870 & 3407.5841 & 0.0000 & 0.0000 & 0.0000 & 0.0000 \\
20139 & 639990.5000 & 480391.6400 & 0.0000 & 0.0000 & 51.1948 & 17.3134 \\
\hline Mean & 97923.1778 & 154198.0548 & 0.0000 & 339.1782 & 10.8681 & 26.8094 \\
STD & 192283.0571 & 205480.0307 & 0 & 988.5483468 & 16.64944839 & 19.43654999 \\
\hline
\end{tabular}

Mean of (seg1\&seg2)

169.5891

18.8387

NHF

\begin{tabular}{|c|cc|cc|cc|}
\hline & \multicolumn{2}{|c|}{ Artifact -Free } & \multicolumn{2}{c|}{ With type 4 \& 5 Artifacts } & \multicolumn{2}{c|}{ Cleaned by Algorithm f } \\
& $\mathrm{seg} 1$ & $\mathrm{seg} 2$ & $\mathrm{seg} 1$ & $\mathrm{seg} 2$ & $\mathrm{seg} 1$ & $\mathrm{seg} 2$ \\
\hline 12042 & 20.1205 & 35.3933 & 0.0000 & 63.3016 & 0.0000 & 3.0632 \\
12043 & 52.8073 & 22.8094 & 0.0000 & 10.0613 & 0.0000 & 19.0032 \\
12064 & 41.1398 & 9.5459 & 0.0000 & 0.0000 & 2.6850 & 9.5945 \\
23020 & 4.6811 & 22.5975 & 0.0000 & 28.1851 & 3.9544 & 4.7997 \\
31039 & 34.4855 & 37.2784 & 0.0000 & 0.0313 & 7.9398 & 1.6539 \\
41070 & 36.8224 & 34.1840 & 0.0000 & 4.4508 & 0.2943 & 1.5458 \\
50070 & 43.7233 & 40.1025 & 0.0000 & 0.0000 & 0.0179 & 19.9887 \\
50077 & 24.0617 & 50.3287 & 0.0000 & 0.0000 & 0.0000 & 1.3606 \\
50098 & 29.7795 & 13.9672 & 0.0000 & 0.0000 & 0.0000 & 0.0000 \\
20139 & 43.8011 & 43.7882 & 0.0000 & 0.0000 & 22.7162 & 4.7367 \\
\hline Mean & 33.1422 & 30.9995 & 0.0000 & 10.6030 & 3.7608 & 6.5746 \\
STD & 14.00380807 & 13.23686239 & 0 & 20.54344509 & 7.153603756 & 7.320441014 \\
\hline
\end{tabular}

Mean of (seg1\&seg2)

5.3015

5.1677

LF

\begin{tabular}{|c|cc|cc|cc|}
\hline & \multicolumn{2}{|c|}{ Artifact -Free } & \multicolumn{2}{c|}{ With type 4 \& 5 Artifacts } & \multicolumn{2}{c|}{ Cleaned by Algorithm f } \\
& $\mathrm{seg} 1$ & $\mathrm{seg} 2$ & $\mathrm{seg} 1$ & $\mathrm{seg} 2$ & $\mathrm{seg} 1$ & $\mathrm{seg} 2$ \\
\hline 12042 & 184251.3200 & 75507.6820 & 0.0000 & 843.9627 & 0.0000 & 49.3498 \\
12043 & 7891.6166 & 51030.6840 & 0.0000 & 1.8339 & 0.0000 & 1.4468 \\
12064 & 136673.6700 & 538090.8800 & 0.0000 & 0.0000 & 38.6989 & 27.5157 \\
23020 & 410346.8400 & 388922.4700 & 0.0000 & 5.3422 & 13.4657 & 34.6651 \\
31039 & 131556.7800 & 37594.0040 & 0.0000 & 0.0122 & 11.3155 & 6.4344 \\
41070 & 136361.6600 & 144931.7900 & 0.0000 & 14.8260 & 0.1158 & 7.1424 \\
50070 & 21501.0570 & 473806.1700 & 0.0000 & 0.0000 & 2.2835 & 37.4193 \\
50077 & 315490.2500 & 697319.9100 & 0.0000 & 0.0000 & 0.0000 & 29.1494 \\
50098 & 96684.3180 & 32953.6020 & 0.0000 & 0.0000 & 0.0000 & 0.0000 \\
20139 & 1186446.2000 & 636690.1200 & 0.0000 & 0.0000 & 22.8221 & 5.6465 \\
\hline Mean & 262720.3712 & 307684.7312 & 0.0000 & 86.5977 & 8.8701 & 19.8769 \\
STD & 347011.9496 & 267090.3324 & 0 & 266.1522497 & 13.1074606 & 17.69979355 \\
\hline
\end{tabular}

Mean of (seg1\&seg2)

43.2989

14.3735 
Table C3 Averaged HRV value for each 15-min segment of each infants (cont.)

NLF

\begin{tabular}{|c|cc|cc|cc|}
\hline & \multicolumn{2}{|c|}{ Artifact - Free } & \multicolumn{2}{c|}{ With type 4 \& 5 Artifacts } & \multicolumn{2}{c|}{ Cleaned by Algorithm f } \\
& seg1 & seg2 & seg1 & seg2 & seg1 & seg2 \\
\hline 12042 & 79.8795 & 64.6067 & 0.0000 & 34.6783 & 0.0000 & 1.6781 \\
12043 & 47.1927 & 77.1906 & 0.0000 & 2.9731 & 0.0000 & 5.6153 \\
12064 & 58.8602 & 90.4541 & 0.0000 & 0.0000 & 1.8766 & 1.0125 \\
23020 & 95.3189 & 77.4025 & 0.0000 & 8.2286 & 0.1942 & 1.4013 \\
31039 & 65.5145 & 62.7216 & 0.0000 & 0.0186 & 4.1793 & 0.9830 \\
41070 & 63.1776 & 65.8160 & 0.0000 & 2.3117 & 0.1715 & 0.8028 \\
50070 & 56.2767 & 59.8975 & 0.0000 & 0.0000 & 0.0139 & 13.3828 \\
50077 & 75.9383 & 49.6713 & 0.0000 & 0.0000 & 0.0000 & 1.3786 \\
50098 & 70.2205 & 86.0328 & 0.0000 & 0.0000 & 0.0000 & 0.0000 \\
20139 & 56.1989 & 56.2118 & 0.0000 & 0.0000 & 17.7049 & 3.6898 \\
\hline Mean & 66.8578 & 69.0005 & 0.0000 & 4.8210 & 2.4141 & 2.9944 \\
STD & 14.00380818 & 13.23686246 & 0 & 10.81180513 & 5.540189041 & 3.998552154 \\
\hline
\end{tabular}

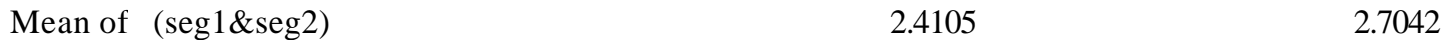

$\mathrm{LF} / \mathrm{HF}$

\begin{tabular}{|c|cc|cc|cc|}
\hline & \multicolumn{2}{|c|}{ Artifact -Free } & \multicolumn{2}{c|}{ With type 4 \& 5 Artifacts } & \multicolumn{2}{c|}{ Cleaned by Algorithm f } \\
& seg1 & seg2 & seg1 & seg2 & seg1 & seg2 \\
\hline 12042 & 10.6585 & 2.0182 & 0.0000 & 57.5045 & 0.0000 & 4.5688 \\
12043 & 0.9483 & 3.5006 & 0.0000 & 6.5917 & 0.0000 & 28.6574 \\
12064 & 3.2188 & 9.9273 & 0.0000 & 0.0000 & 20.6288 & 11.2627 \\
23020 & 20.6523 & 8.4114 & 0.0000 & 28.8629 & 5.0899 & 6.9655 \\
31039 & 1.9395 & 1.9425 & 0.0000 & 0.0225 & 11.1059 & 3.5904 \\
41070 & 3.3845 & 2.4788 & 0.0000 & 20.2952 & 1.1062 & 17.3394 \\
50070 & 1.4746 & 1.7674 & 0.0000 & 0.0000 & 0.1850 & 83.2287 \\
50077 & 3.4119 & 1.1659 & 0.0000 & 0.0000 & 0.0000 & 21.4084 \\
50098 & 2.5368 & 13.3001 & 0.0000 & 0.0000 & 0.0000 & 0.0000 \\
20139 & 1.5323 & 1.3035 & 0.0000 & 0.0000 & 103.4026 & 10.0459 \\
\hline Mean & 4.9757 & 4.5816 & 0.0000 & 11.3277 & 14.1518 & 18.7067 \\
STD & 6.160945247 & 4.329684053 & 0 & 19.1833756 & 32.08565211 & 24.30791881 \\
\hline
\end{tabular}

Mean of (seg1\&seg2)

5.6638

16.4293

Total-Power

\begin{tabular}{|c|c|c|c|c|c|c|}
\hline & \multicolumn{2}{|c|}{ Artifact - Free } & \multicolumn{2}{|c|}{ With type $4 \& 5$ Artifacts } & \multicolumn{2}{|c|}{ Cleaned by Algorithm f } \\
\hline & $\operatorname{seg} 1$ & $\operatorname{seg} 2$ & seg 1 & $\operatorname{seg} 2$ & seg1 & seg2 \\
\hline 12042 & 199785.8400 & 114465.7500 & 0.0000 & 1628.4296 & 0.0000 & 44.6472 \\
\hline 12043 & 16029.8100 & 67931.4670 & 0.0000 & 8.4658 & 0.0000 & 7.8823 \\
\hline 12064 & 171502.6800 & 591996.9400 & 0.0000 & 0.0000 & 33.0900 & 26.4066 \\
\hline 23020 & 430558.9400 & 424142.0700 & 0.0000 & 18.4683 & 13.6776 & 34.9391 \\
\hline 31039 & 203333.7800 & 59453.2270 & 0.0000 & 0.0399 & 15.9487 & 6.9456 \\
\hline 41070 & 177245.1300 & 208176.7200 & 0.0000 & 25.7919 & 0.0551 & 1.3235 \\
\hline 50070 & 39078.8760 & 758073.9800 & 0.0000 & 0.0000 & 2.7675 & 47.1974 \\
\hline 50077 & 408089.0200 & 1241144.8000 & 0.0000 & 0.0000 & 0.0000 & 35.0276 \\
\hline 50098 & 134374.7000 & 36361.1860 & 0.0000 & 0.0000 & 0.0000 & 0.0000 \\
\hline 20139 & 1826436.7000 & 1117081.8000 & 0.0000 & 0.0000 & 32.7640 & 10.6637 \\
\hline Mean & 360643.5476 & 461882.7940 & 0.0000 & 168.1196 & 9.8303 & 21.5033 \\
\hline STD & 532194.5833 & 450419.496 & 0 & 513.1838626 & 13.54490878 & 18.1594018 \\
\hline
\end{tabular}


APPENDIX D

RESULT FOR HRV CORRELATION STUDY 
Table 5.1 Combinations of HRV parameters that has very strong correlation

\begin{tabular}{|c|c|c|c|c|c|c|c|c|c|c|c|c|c|c|c|c|c|c|}
\hline \multicolumn{19}{|c|}{ Mean -- Median } \\
\hline Stat & \multicolumn{3}{|c|}{ individual } & \multicolumn{3}{|c|}{ Bootstrap-All Infants } & \multicolumn{3}{|c|}{ Bootstrap-ALTE } & \multicolumn{3}{|c|}{ Bootstrap-Preterm } & \multicolumn{3}{|c|}{ Bootstrap-Siblings } & \multicolumn{3}{|c|}{ Bootstrap-Healthy } \\
\hline & QS & REM & AWK & QS & REM & AWK & QS & REM & AWK & QS & REM & AWK & QS & REM & AWK & QS & REM & AWK \\
\hline $2.5 \%$ & 0.9462 & 0.9080 & 0.9009 & 0.9985 & 0.9953 & 0.9765 & 0.9978 & 0.9944 & 0.9493 & 0.9979 & 0.9919 & 0.9825 & 0.9969 & 0.9934 & 0.9784 & 0.9962 & 0.9912 & 0.9777 \\
\hline $25.0 \%$ & 0.9879 & 0.9650 & 0.9739 & 0.9987 & 0.9958 & 0.9844 & 0.9981 & 0.9950 & 0.9607 & 0.9983 & 0.9928 & 0.9853 & 0.9976 & 0.9942 & 0.9841 & 0.9966 & 0.9932 & 0.9832 \\
\hline $50.0 \%$ & 0.9938 & 0.9826 & 0.9838 & 0.9988 & 0.9960 & 0.9870 & 0.9985 & 0.9953 & 0.9837 & 0.9985 & 0.9933 & 0.9868 & 0.9980 & 0.9950 & 0.9871 & 0.9969 & 0.9941 & 0.9858 \\
\hline $75.0 \%$ & 0.9975 & 0.9904 & 0.9903 & 0.9989 & 0.9962 & 0.9883 & 0.9986 & 0.9956 & 0.9877 & 0.9987 & 0.9937 & 0.9880 & 0.9983 & 0.9954 & 0.9891 & 0.9971 & 0.9947 & 0.9880 \\
\hline $97.5 \%$ & 0.9994 & 0.9980 & 0.9967 & 0.9991 & 0.9966 & 0.9903 & 0.9988 & 0.9962 & 0.9915 & 0.9989 & 0.9946 & 0.9897 & 0.9987 & 0.9961 & 0.9919 & 0.9975 & 0.9957 & 0.9907 \\
\hline std & 0.0138 & 0.0246 & 0.0342 & 0.0002 & 0.0003 & 0.0040 & 0.0003 & 0.0005 & 0.0143 & 0.0003 & 0.0007 & 0.0019 & 0.0005 & 0.0008 & 0.0036 & 0.0003 & 0.0011 & 0.0035 \\
\hline & & & & & & & & & & & & & & & & & $\begin{array}{c}0.9009 \\
\min \end{array}$ & $\begin{array}{c}0.9994 \\
\max \end{array}$ \\
\hline
\end{tabular}

SDNN -- CV

\begin{tabular}{|c|c|c|c|c|c|c|c|c|c|c|c|c|c|c|c|c|c|c|}
\hline \multirow[t]{2}{*}{ Stat } & \multicolumn{3}{|c|}{ individual } & \multicolumn{3}{|c|}{ Bootstrap-All Infants } & \multicolumn{3}{|c|}{ Bootstrap-ALTE } & \multicolumn{3}{|c|}{ Bootstrap-Preterm } & \multicolumn{3}{|c|}{ Bootstrap-Siblings } & \multicolumn{3}{|c|}{ Bootstrap-Healthy } \\
\hline & QS & REM & AWK & QS & REM & AWK & QS & REM & AWK & QS & REM & AWK & QS & REM & AWK & QS & REM & AWK \\
\hline $2.5 \%$ & 0.9715 & 0.9508 & 0.9105 & 0.9750 & 0.9643 & 0.9549 & 0.9706 & 0.9573 & 0.9542 & 0.9831 & 0.9786 & 0.9572 & 0.9684 & 0.9555 & 0.9465 & 0.9799 & 0.9724 & 0.9560 \\
\hline $25.0 \%$ & 0.9902 & 0.9806 & 0.9657 & 0.9776 & 0.9668 & 0.9611 & 0.9738 & 0.9625 & 0.9638 & 0.9852 & 0.9804 & 0.9627 & 0.9718 & 0.9613 & 0.9580 & 0.9818 & 0.9793 & 0.9633 \\
\hline $50.0 \%$ & 0.9946 & 0.9911 & 0.9762 & 0.9788 & 0.9680 & 0.9666 & 0.9754 & 0.9651 & 0.9687 & 0.9862 & 0.9815 & 0.9657 & 0.9741 & 0.9639 & 0.9676 & 0.9830 & 0.9840 & 0.9669 \\
\hline $75.0 \%$ & 0.9975 & 0.9952 & 0.9877 & 0.9800 & 0.9693 & 0.9754 & 0.9769 & 0.9676 & 0.9937 & 0.9871 & 0.9824 & 0.9746 & 0.9765 & 0.9662 & 0.9810 & 0.9839 & 0.9862 & 0.9701 \\
\hline $97.5 \%$ & 0.9993 & 0.9987 & 0.9984 & 0.9820 & 0.9716 & 0.9882 & 0.9792 & 0.9717 & 0.9955 & 0.9887 & 0.9839 & 0.9788 & 0.9802 & 0.9701 & 0.9857 & 0.9858 & 0.9891 & 0.9754 \\
\hline std & 0.0071 & 0.0152 & 0.0282 & 0.0018 & 0.0018 & 0.0105 & 0.0022 & 0.0037 & 0.0145 & 0.0014 & 0.0014 & 0.0066 & 0.0032 & 0.0037 & 0.0123 & 0.0015 & 0.0045 & 0.0050 \\
\hline & & & & & & & & & & & & & & & & & $\begin{array}{c}0.9105 \\
\min \end{array}$ & $\begin{array}{c}0.9993 \\
\max \end{array}$ \\
\hline
\end{tabular}

IQRNN -- NIQRNN

\begin{tabular}{|c|c|c|c|c|c|c|c|c|c|c|c|c|c|c|c|c|c|c|}
\hline \multirow[t]{2}{*}{ Stat } & \multicolumn{3}{|c|}{ individual } & \multicolumn{3}{|c|}{ Bootstrap-All Infants } & \multicolumn{3}{|c|}{ Bootstrap-ALTE } & \multicolumn{3}{|c|}{ Bootstrap-Preterm } & \multicolumn{3}{|c|}{ Bootstrap-Siblings } & \multicolumn{3}{|c|}{ Bootstrap-Healthy } \\
\hline & QS & REM & AWK & QS & REM & AWK & QS & REM & AWK & QS & REM & AWK & QS & REM & AWK & QS & REM & AWK \\
\hline $2.5 \%$ & 0.9707 & 0.9738 & 0.9456 & 0.9781 & 0.9737 & 0.9653 & 0.9763 & 0.9714 & 0.9659 & 0.9842 & 0.9845 & 0.9649 & 0.9690 & 0.9604 & 0.9619 & 0.9826 & 0.9745 & 0.9663 \\
\hline $25.0 \%$ & 0.9924 & 0.9880 & 0.9773 & 0.9807 & 0.9759 & 0.9691 & 0.9794 & 0.9764 & 0.9716 & 0.9868 & 0.9865 & 0.9687 & 0.9730 & 0.9658 & 0.9698 & 0.9845 & 0.9825 & 0.9743 \\
\hline $50.0 \%$ & 0.9957 & 0.9947 & 0.9843 & 0.9821 & 0.9772 & 0.9708 & 0.9807 & 0.9786 & 0.9750 & 0.9879 & 0.9874 & 0.9706 & 0.9752 & 0.9685 & 0.9728 & 0.9855 & 0.9884 & 0.9775 \\
\hline $75.0 \%$ & 0.9974 & 0.9975 & 0.9894 & 0.9833 & 0.9784 & 0.9724 & 0.9820 & 0.9804 & 0.9778 & 0.9890 & 0.9883 & 0.9723 & 0.9782 & 0.9710 & 0.9759 & 0.9869 & 0.9904 & 0.9804 \\
\hline $97.5 \%$ & 0.9994 & 0.9996 & 0.9974 & 0.9854 & 0.9803 & 0.9750 & 0.9842 & 0.9832 & 0.9817 & 0.9905 & 0.9898 & 0.9752 & 0.9847 & 0.9753 & 0.9810 & 0.9884 & 0.9927 & 0.9846 \\
\hline std & 0.0085 & 0.0091 & 0.0139 & 0.0019 & 0.0018 & 0.0025 & 0.0020 & 0.0030 & 0.0043 & 0.0016 & 0.0013 & 0.0026 & 0.0042 & 0.0038 & 0.0048 & 0.0016 & 0.0051 & 0.0047 \\
\hline
\end{tabular}


Table 5.1 Combinations of HRV parameters that has very strong correlation (cont.) IQRSD -- NIQRSD

\begin{tabular}{|c|c|c|c|c|c|c|c|c|c|c|c|c|c|c|c|c|c|c|}
\hline Stat & \multicolumn{3}{|c|}{ individual } & \multicolumn{3}{|c|}{ Bootstrap-All Infants } & \multicolumn{3}{|c|}{ Bootstrap-ALTE } & \multicolumn{3}{|c|}{ Bootstrap-Preterm } & \multicolumn{3}{|c|}{ Bootstrap-Siblings } & \multicolumn{3}{|c|}{ Bootstrap-Healthy } \\
\hline & QS & REM & AWK & QS & REM & AWK & QS & REM & AWK & QS & REM & AWK & QS & REM & AWK & QS & REM & AWK \\
\hline $2.5 \%$ & 0.9662 & 0.9549 & 0.8616 & 0.9823 & 0.9799 & 0.9571 & 0.9815 & 0.9800 & 0.9452 & 0.9848 & 0.9870 & 0.9667 & 0.9800 & 0.9736 & 0.9396 & 0.990 & 0.9911 & 0.9566 \\
\hline $25.0 \%$ & 0.9883 & 0.9867 & 0.9556 & 0.9837 & 0.9812 & 0.9612 & 0.9832 & 0.9822 & 0.9526 & 0.9862 & 0.9881 & 0.9705 & 0.9820 & 0.9766 & 0.9522 & 0.991 & 0.9925 & 0.9654 \\
\hline $50.0 \%$ & 0.9952 & 0.9938 & 0.9731 & 0.9844 & 0.9818 & 0.9630 & 0.9842 & 0.9833 & 0.9568 & 0.9870 & 0.9886 & 0.9724 & 0.9829 & 0.9780 & 0.9592 & 0.99 & 0.9932 & 0.9695 \\
\hline $75.0 \%$ & 0.9976 & 0.9968 & 0.9859 & 0.9852 & 0.9824 & 0.9647 & 0.9850 & 0.9843 & 0.9604 & 0.9877 & 0.9892 & 0.9741 & 0.9839 & 0.9793 & 0.9651 & 0.992 & 0.9938 & 0.9734 \\
\hline $97.5 \%$ & 0.9996 & 0.9994 & 0.9964 & 0.9867 & 0.9836 & 0.9677 & 0.9868 & 0.9860 & 0.9676 & 0.9892 & 0.9903 & 0.9768 & 0.9856 & 0.9817 & 0.9734 & 0.993 & 0.9950 & 0.9805 \\
\hline std & 0.0083 & 0.0110 & 0.0343 & 0.0011 & 0.0009 & 0.0026 & 0.0013 & 0.0016 & 0.0057 & 0.0011 & 0.0008 & 0.0026 & 0.0014 & 0.0020 & 0.0089 & 0.000 & 0.0010 & 0.0061 \\
\hline
\end{tabular}

RMSSD -- CVS

\begin{tabular}{|c|c|c|c|c|c|c|c|c|c|c|c|c|c|c|c|c|c|c|}
\hline Stat & \multicolumn{3}{|c|}{ individual } & \multicolumn{3}{|c|}{ Bootstrap-All Infants } & \multicolumn{3}{|c|}{ Bootstrap-ALTE } & \multicolumn{3}{|c|}{ Bootstrap-Preterm } & \multicolumn{3}{|c|}{ Bootstrap-Siblings } & \multicolumn{3}{|c|}{ Bootstrap-Healthy } \\
\hline & QS & REM & AWK & $\mathrm{QS}$ & REM & AWK & $\mathrm{QS}$ & REM & AWK & $\mathrm{QS}$ & REM & AWK & $\mathrm{QS}$ & REM & AWK & QS & REM & AWK \\
\hline $2.5 \%$ & 0.9703 & 0.9437 & 0.8498 & 0.9824 & 0.9774 & 0.9431 & 0.9837 & 0.9777 & 0.9308 & 0.9835 & 0.9844 & 0.9533 & 0.9825 & 0.9748 & 0.9208 & 0.990 & 30.9905 & 0.9471 \\
\hline $25.0 \%$ & 0.9875 & 0.9821 & 0.9422 & 0.9837 & 0.9789 & 0.9484 & 0.9849 & 0.9800 & 0.9433 & 0.9851 & 0.9857 & 0.9578 & 0.9843 & 0.9777 & 0.9358 & 0.991 & 20.9919 & 0.9560 \\
\hline $50.0 \%$ & 0.9944 & 0.9918 & 0.9707 & 0.9844 & 0.9796 & 0.9510 & 0.9857 & 0.9811 & 0.9501 & 0.9859 & 0.9862 & 0.9599 & 0.9851 & 0.9789 & 0.9445 & 0.99 & 0.9927 & 0.9620 \\
\hline $75.0 \%$ & 0.9972 & 0.9959 & 0.9844 & 0.9851 & 0.9803 & 0.9538 & 0.9864 & 0.9823 & 0.9596 & 0.9868 & 0.9868 & 0.9621 & 0.9859 & 0.9801 & 0.9517 & 0.992 & 0.9934 & 0.9666 \\
\hline $97.5 \%$ & 0.9995 & 0.9994 & 0.9957 & 0.9865 & 0.9815 & 0.9588 & 0.9878 & 0.9841 & 0.9686 & 0.9882 & 0.9879 & 0.9653 & 0.9875 & 0.9822 & 0.9625 & 0.993 & 0.9945 & 0.9751 \\
\hline std & 0.0087 & 0.0131 & 0.0399 & 0.0011 & 0.0010 & 0.0040 & 0.0011 & 0.0016 & 0.0104 & 0.0012 & 0.0009 & 0.0031 & 0.0012 & 0.0018 & 0.0111 & 0.000 & 70.0011 & 0.0075 \\
\hline
\end{tabular}

RMSSD -- SDSD

\begin{tabular}{|c|c|c|c|c|c|c|c|c|c|c|c|c|c|c|c|c|c|c|}
\hline Stat & \multicolumn{3}{|c|}{ individual } & \multicolumn{3}{|c|}{ Bootstrap-All Infants } & \multicolumn{3}{|c|}{ Bootstrap-ALTE } & \multicolumn{3}{|c|}{ Bootstrap-Preterm } & \multicolumn{3}{|c|}{ Bootstrap-Siblings } & \multicolumn{3}{|c|}{ Bootstrap-Healthy } \\
\hline & $\mathrm{QS}$ & REM & AWK & QS & REM & AWK & QS & REM & AWK & QS & REM & AWK & QS & REM & $\mathrm{AWK}$ & QS & REM & $\mathrm{AWK}$ \\
\hline $2.5 \%$ & 0.8165 & 0.8755 & 0.8486 & 0.9740 & 0.9787 & 0.9615 & 0.9828 & 0.9806 & 0.9609 & 0.9541 & 0.9692 & 0.9645 & 0.9742 & 0.9896 & 0.9529 & 0.9777 & 0.9875 & 0.9548 \\
\hline $25.0 \%$ & 0.9414 & .9679 & 9572 & 9772 & .9809 & 9657 & 14 & 0.9832 & 0.9677 & .9624 & 0.9730 & 0.9692 & 0.9781 & 0.9910 & 0.9605 & & & 0.9650 \\
\hline $75.0 \%$ & 0.9864 & 0.9896 & 0.9873 & 0.9801 & 0.9831 & 0.9696 & 0.9861 & 0.9860 & 0.9743 & 0.9705 & 0.9766 & 0.9735 & 0.9815 & 0.9924 & 0.9678 & 0.98 & 0.9918 & 0.9740 \\
\hline $97.5 \%$ & 0.9961 & 0.9970 & 0.9969 & 0.9828 & 0.9851 & 0.9733 & 0.9879 & 0.9882 & 0.9790 & 0.9775 & 0.9803 & 0.9774 & 0.9841 & 0.9937 & 0.9735 & 0.98 & 0.9935 & 0.9799 \\
\hline std & 0.0464 & 0.0586 & 0.0505 & 0.0022 & 0.0017 & 0.0030 & 0.0013 & 0.0020 & 0.0048 & 0.0060 & 0.0028 & 0.0032 & 0.0025 & 0.0011 & 0.0053 & 0.0018 & 0.0015 & 0.0065 \\
\hline
\end{tabular}

SDNN -- IQRNN

\begin{tabular}{|c|c|c|c|c|c|c|c|c|c|c|c|c|c|c|c|c|c|c|}
\hline Stat & \multicolumn{3}{|c|}{ individual } & \multicolumn{3}{|c|}{ Bootstrap-All Infants } & \multicolumn{3}{|c|}{ Bootstrap-ALTE } & \multicolumn{3}{|c|}{ Bootstrap-Preterm } & \multicolumn{3}{|c|}{ Bootstrap-Siblings } & \multicolumn{3}{|c|}{ Bootstrap-Healthy } \\
\hline & QS & REM & AWK & QS & REM & AWK & QS & REM & AWK & QS & REM & AWK & QS & REM & AWK & QS & REM & AWK \\
\hline $2.5 \%$ & 0.5829 & 0.4384 & 0.1620 & 0.9169 & 0.9028 & 0.3809 & 0.9099 & 0.8559 & 0.1817 & 0.9116 & 0.9034 & 0.5606 & 0.8953 & 0.8975 & 0.4180 & 0.8677 & 0.8816 & 0.8654 \\
\hline $25.0 \%$ & 0.8369 & 0.7945 & 0.7724 & 0.9269 & 0.9115 & 0.6288 & 0.9203 & 0.8729 & 0.2511 & 0.9266 & 0.9138 & 0.6289 & 0.9133 & 0.9111 & 0.5360 & 0.8818 & 0.8964 & 0.8967 \\
\hline $50.0 \%$ & 0.9033 & 0.8699 & 0.8735 & 0.9318 & 0.9154 & 0.7502 & 0.9257 & 0.8807 & 0.8442 & 0.9335 & 0.9200 & 0.7589 & 0.9215 & 0.9177 & 0.7962 & 0.88 & 0.9033 & 0.9115 \\
\hline $75.0 \%$ & 0.9419 & 0.9194 & 0.9165 & 0.9366 & 0.9195 & 0.8292 & 0.9309 & 0.8883 & 0.8802 & 0.9410 & 0.9257 & 0.7959 & 0.9307 & 0.9235 & 0.8837 & 0.89 & 0.9106 & 0.9239 \\
\hline $97.5 \%$ & 0.9871 & 0.9788 & 0.9611 & 0.9457 & 0.9263 & 0.8734 & 0.9396 & 0.9017 & 0.9199 & 0.9528 & 0.9361 & 0.8411 & 0.9437 & 0.9345 & 0.9189 & 0.906 & 0.9249 & 0.9404 \\
\hline std & 0.0991 & 0.1476 & 0.2055 & 0.0073 & 0.0060 & 0.1643 & 0.0077 & 0.0116 & 0.3016 & 0.0108 & 0.0085 & 0.0898 & 0.0126 & 0.0093 & 0.1759 & 0.0101 & 0.0111 & 0.0196 \\
\hline
\end{tabular}


Table 5.2 Highly correlated HRV parameters that measures vagal activity

RMSSD -- IQRSD

\begin{tabular}{|c|c|c|c|c|c|c|c|c|c|c|c|c|c|c|c|c|c|c|}
\hline \multirow[t]{2}{*}{ Stat } & \multicolumn{3}{|c|}{ individual } & \multicolumn{3}{|c|}{ Bootstrap-All Infants } & \multicolumn{3}{|c|}{ Bootstrap-ALTE } & \multicolumn{3}{|c|}{ Bootstrap-Preterm } & \multicolumn{3}{|c|}{ Bootstrap-Siblings } & \multicolumn{3}{|c|}{ Bootstrap-Healthy } \\
\hline & QS & REM & AWK & QS & REM & AWK & QS & REM & AWK & QS & REM & AWK & QS & REM & AWK & QS & REM & $\mathrm{AWK}$ \\
\hline $2.5 \%$ & 0.3696 & 0.4593 & 0.3300 & 0.9714 & 0.9534 & 0.7771 & 0.9691 & 0.9316 & 0.6529 & 0.9488 & 0.9374 & 0.8111 & 0.9709 & 0.9662 & 0.6326 & 0.9763 & 0.9685 & 0.9195 \\
\hline $25.0 \%$ & 0.8501 & 0.7987 & 0.6931 & 0.9747 & 0.9577 & 0.8324 & 0.9726 & 0.9410 & 0.7079 & 0.9579 & 0.9446 & 0.8319 & 0.9761 & 0.9703 & 0.7452 & 0.9793 & 0.9734 & 0.9349 \\
\hline $50.0 \%$ & 0.9258 & 0.8927 & 0.8131 & 0.9767 & 0.9601 & 0.8545 & 0.9745 & 0.9462 & 0.8794 & 0.9623 & 0.9481 & 0.8412 & 0.9783 & 0.9724 & 0.8146 & 0.9807 & 0.9755 & 0.9427 \\
\hline $75.0 \%$ & 0.9571 & 0.9501 & 0.9061 & 0.9785 & 0.9623 & 0.8677 & 0.9765 & 0.9512 & 0.8937 & 0.9663 & 0.9517 & 0.8498 & 0.9804 & 0.9744 & 0.8803 & 0.9822 & 0.9776 & 0.9496 \\
\hline $97.5 \%$ & 0.9917 & 0.9915 & 0.9798 & 0.9815 & 0.9659 & 0.8845 & 0.9802 & 0.9603 & 0.9102 & 0.9730 & 0.9575 & 0.8672 & 0.9836 & 0.9780 & 0.9200 & 0.9847 & 0.9812 & 0.9619 \\
\hline std & 0.1382 & 0.1329 & 0.1833 & 0.0026 & 0.0032 & 0.0307 & 0.0028 & 0.0073 & 0.0941 & 0.0062 & 0.0052 & 0.0143 & 0.0033 & 0.0031 & 0.0828 & 0.0022 & 0.0031 & 0.0110 \\
\hline & & & & & & & & & & & & & & & & & $\begin{array}{c}0.33 \\
\min \end{array}$ & $\begin{array}{c}0.9917 \\
\max \end{array}$ \\
\hline
\end{tabular}

RMSSD -- pNN50

\begin{tabular}{|c|c|c|c|c|c|c|c|c|c|c|c|c|c|c|c|c|c|c|}
\hline \multirow[t]{2}{*}{ Stat } & \multicolumn{3}{|c|}{ individual } & \multicolumn{3}{|c|}{ Bootstrap-All Infants } & \multicolumn{3}{|c|}{ Bootstrap-ALTE } & \multicolumn{3}{|c|}{ Bootstrap-Preterm } & \multicolumn{3}{|c|}{ Bootstrap-Siblings } & \multicolumn{3}{|c|}{ Bootstrap-Healthy } \\
\hline & QS & REM & AWK & QS & REM & AWK & QS & REM & AWK & QS & REM & AWK & QS & REM & AWK & QS & REM & AWK \\
\hline $2.5 \%$ & -0.1018 & -0.2428 & -0.2837 & 0.7168 & 0.8181 & 0.7342 & 0.7543 & 0.7653 & 0.6908 & 0.5618 & 0.7719 & 0.7423 & 0.7382 & 0.8757 & 0.7330 & 0.7966 & 0.8501 & 0.5896 \\
\hline $25.0 \%$ & 0.1101 & 0.2380 & 0.1375 & 0.7402 & 0.8284 & 0.7563 & 0.7740 & 0.7939 & 0.7228 & 0.6101 & 0.7863 & 0.7644 & 0.7751 & 0.8883 & 0.7847 & 0.8120 & 0.8656 & 0.6695 \\
\hline $50.0 \%$ & 0.4326 & 0.5583 & 0.4409 & 0.7518 & 0.8336 & 0.7668 & 0.7845 & 0.8081 & 0.7401 & 0.6331 & 0.7938 & 0.7756 & 0.7934 & 0.8949 & 0.8104 & 0.8204 & 0.8725 & 0.7045 \\
\hline $75.0 \%$ & 0.6819 & 0.7984 & 0.6379 & 0.7626 & 0.8387 & 0.7771 & 0.7934 & 0.8232 & 0.7554 & 0.6558 & 0.8023 & 0.7850 & 0.8098 & 0.9011 & 0.8332 & 0.8284 & 0.8788 & 0.7365 \\
\hline $97.5 \%$ & 0.9678 & 0.9635 & 0.9004 & 0.7803 & 0.8481 & 0.7953 & 0.8101 & 0.8501 & 0.7852 & 0.6950 & 0.8182 & 0.8033 & 0.8339 & 0.9121 & 0.8697 & 0.8416 & 0.8910 & 0.7816 \\
\hline std & $\mathrm{NaN}$ & $\mathrm{NaN}$ & 0.3220 & 0.0167 & 0.0077 & 0.0154 & 0.0143 & 0.0221 & 0.0243 & 0.0342 & 0.0119 & 0.0155 & 0.0248 & 0.0092 & 0.0354 & 0.0117 & 0.0102 & 0.0505 \\
\hline
\end{tabular}

RMSSD -- HF

\begin{tabular}{|c|c|c|c|c|c|c|c|c|c|c|c|c|c|c|c|c|c|c|}
\hline \multirow[t]{2}{*}{ Stat } & \multicolumn{3}{|c|}{ individual } & \multicolumn{3}{|c|}{ Bootstrap-All Infants } & \multicolumn{3}{|c|}{ Bootstrap-ALTE } & \multicolumn{3}{|c|}{ Bootstrap-Preterm } & \multicolumn{3}{|c|}{ Bootstrap-Siblings } & \multicolumn{3}{|c|}{ Bootstrap-Healthy } \\
\hline & QS & REM & AWK & QS & REM & A WK & QS & REM & AWK & QS & REM & AWK & QS & REM & AWK & QS & REM & AWK \\
\hline $2.5 \%$ & 0.4985 & 0.2595 & 0.3359 & 0.8596 & 0.8504 & 0.4943 & 0.9083 & 0.7938 & 0.6027 & 0.8213 & 0.8442 & 0.6453 & 0.8740 & 0.9032 & 0.5286 & 0.8802 & 0.8097 & 0.7363 \\
\hline $25.0 \%$ & 0.7780 & 0.6303 & 0.6264 & 0.8778 & 0.8624 & 0.7055 & 0.9163 & 0.8303 & 0.6541 & 0.8431 & 0.8594 & 0.6955 & 0.8958 & 0.9132 & 0.6723 & 0.8929 & 0.8452 & 0.7891 \\
\hline $50.0 \%$ & 0.8823 & 0.7776 & 0.7653 & 0.8881 & 0.8690 & 0.7428 & 0.9201 & 0.8453 & 0.7806 & 0.8606 & 0.8670 & 0.7238 & 0.9062 & 0.9184 & 0.7610 & 0.8993 & 0.8633 & 0.8225 \\
\hline $75.0 \%$ & 0.9355 & 0.8685 & 0.8487 & 0.8969 & 0.8748 & 0.7706 & 0.9237 & 0.8605 & 0.8046 & 0.8851 & 0.8756 & 0.7551 & 0.9167 & 0.9240 & 0.8161 & 0.9066 & 0.8818 & 0.8482 \\
\hline $97.5 \%$ & 0.9873 & 0.9592 & 0.9262 & 0.9088 & 0.8872 & 0.8067 & 0.9306 & 0.8843 & 0.8338 & 0.9084 & 0.8895 & 0.7906 & 0.9318 & 0.9347 & 0.8675 & 0.9194 & 0.9081 & 0.8816 \\
\hline std & 0.1228 & 0.1885 & 0.1584 & 0.0132 & 0.0093 & 0.0838 & 0.0056 & 0.0229 & 0.0793 & 0.0252 & 0.0118 & 0.0388 & 0.0151 & 0.0080 & 0.0955 & 0.0100 & 0.0260 & 0.0396 \\
\hline
\end{tabular}


Table 5.2 Highly correlated HRV parameters that measures vagal activity (cont.)

RMSSD -- NHF

\begin{tabular}{|c|c|c|c|c|c|c|c|c|c|c|c|c|c|c|c|c|c|c|}
\hline \multirow[t]{2}{*}{ Stat } & \multicolumn{3}{|c|}{ individual } & \multicolumn{3}{|c|}{ Bootstrap-All Infants } & \multicolumn{3}{|c|}{ Bootstrap-ALTE } & \multicolumn{3}{|c|}{ Bootstrap-Preterm } & \multicolumn{3}{|c|}{ Bootstrap-Siblings } & \multicolumn{3}{|c|}{ Bootstrap-Healthy } \\
\hline & QS & REM & AWK & $\mathrm{QS}$ & REM & & QS & & AWK & QS & REM & AWK & QS & REM & & QS & REM & AWK \\
\hline $2.5 \%$ & -0.473 & & & .4396 & & & & & & & 0.2485 & 0.2341 & & & & 0.4037 & & 0.1601 \\
\hline $25.0 \%$ & -0.1477 & 0.0243 & 0 . & 0.4711 & 662 & & & & & 0.3999 & 0.2848 & 0.2743 & 0.3315 & 0.4322 & 0.1084 & 0.4437 & 0.3 & 0.2720 \\
\hline $50.0 \%$ & 0.1479 & 0.2701 & 0.2301 & 0.4875 & & 824 & & & 306 & 0.4286 & 0.3047 & 0.2991 & 0.3628 & 614 & 0.1621 & 0.4621 & 0.3831 & 0.3293 \\
\hline $75.0 \%$ & 0.4009 & 0.5019 & 0.4294 & 0.5049 & 0.3971 & 0.3082 & 0.5742 & 0.6174 & 0.3807 & 0.4545 & 0.3230 & 0.3189 & 0.3923 & 0.4902 & 0.2189 & 0.4844 & 0.4174 & 0.3860 \\
\hline $97.5 \%$ & 0.8834 & 0.7702 & 0.7149 & 0.5332 & 0.4279 & 0.3484 & 0.6032 & 0.6687 & 0.4480 & 0.5028 & 0.3588 & 0.3579 & 0.4516 & 0.5435 & 0.3287 & 0.5208 & 0.4735 & 0.4806 \\
\hline std & 0.3532 & 0.3409 & 0.3022 & 0.0238 & 0.0231 & 0.0340 & 0.0234 & 0.0424 & 0.0727 & 0.0391 & 0.0287 & 0.0323 & 0.0455 & 0.0438 & 0.0824 & 0.0297 & 0.0536 & 0.0807 \\
\hline
\end{tabular}

RMSSD -- HFW

\begin{tabular}{|c|c|c|c|c|c|c|c|c|c|c|c|c|c|c|c|c|c|c|}
\hline Stat & \multicolumn{3}{|c|}{ individual } & \multicolumn{3}{|c|}{ Bootstrap-All Infants } & \multicolumn{3}{|c|}{ Bootstrap-ALTE } & \multicolumn{3}{|c|}{ Bootstrap-Preterm } & \multicolumn{3}{|c|}{ Bootstrap-Siblings } & \multicolumn{3}{|c|}{ Bootstrap-Healthy } \\
\hline & QS & REM & AWK & QS & REM & AWK & QS & REM & AWK & QS & REM & AWK & QS & REM & AWK & QS & REM & AWK \\
\hline $2.5 \%$ & 0.7670 & 0.7119 & 0.7108 & 0.8885 & 0.9143 & 0.7310 & 0.9235 & 08043 & 0.8336 & 0.8613 & 0.9039 & 0.7763 & 0.9440 & 0.9468 & 0.6714 & 0.9141 & 0.9196 & 0.8652 \\
\hline $25.0 \%$ & 0.9196 & 0.9006 & 0.8574 & 0.9035 & 0.9193 & 0.8433 & 0.9293 & 0.9066 & 0.8490 & 0.8739 & 0.9105 & 0.8310 & 0.9514 & 0.9524 & 0.8326 & 0.9214 & 0.9271 & 0.8893 \\
\hline $50.0 \%$ & 0.9561 & 0.9326 & 0.9036 & 0.9117 & 0.9218 & 0.8644 & 0.9327 & 0.9135 & 0.8592 & 0.8823 & 0.9143 & 0.8523 & 0.9548 & 0.9551 & 0.8751 & 0.9267 & 0.9307 & 0.9014 \\
\hline $75.0 \%$ & 0.9770 & 0.9671 & 0.9440 & 0.9202 & 0.9244 & 0.8745 & 0.9361 & 0.9211 & 0.8978 & 0.8907 & 0.9179 & 0.8655 & 0.9578 & 0.9577 & 0.9030 & 0.9319 & 0.9344 & 0.9123 \\
\hline $97.5 \%$ & 0.9930 & 0.9952 & 0.9785 & 0.9285 & 0.9303 & 0.8941 & 0.9418 & 0.9340 & 0.9166 & 0.9134 & 0.9243 & 0.8867 & 0.9633 & 0.9622 & 0.9243 & 0.9444 & 0.9447 & 0.9287 \\
\hline std & 0.0576 & 0.0833 & 0.0744 & 0.0110 & 0.0039 & 0.0433 & 0.0048 & 0.0101 & 0.0263 & 0.0131 & 0.0053 & 0.0315 & 0.0047 & 0.0039 & 0.0728 & 0.0077 & 0.0060 & 0.0166 \\
\hline
\end{tabular}

SDSD -- IQRSD

\begin{tabular}{|c|c|c|c|c|c|c|c|c|c|c|c|c|c|c|c|c|c|c|}
\hline \multirow[t]{2}{*}{ Stat } & \multicolumn{3}{|c|}{ individual } & \multicolumn{3}{|c|}{ Bootstrap-All Infants } & \multicolumn{3}{|c|}{ Bootstrap-ALTE } & \multicolumn{3}{|c|}{ Bootstrap-Preterm } & \multicolumn{3}{|c|}{ Bootstrap-Siblings } & \multicolumn{3}{|c|}{ Bootstrap-Healthy } \\
\hline & QS & REM & AWK & QS & REM & AWK & QS & REM & AWK & QS & REM & AWK & QS & REM & AWK & QS & REM & AWK \\
\hline $2.5 \%$ & 0.3448 & 0.3593 & 0.1525 & 0.9391 & 0.8926 & 0.6163 & 0.9359 & 0.8579 & 0.4834 & 0.9042 & 0.8529 & 0.6639 & 0.9349 & 0.9329 & 0.4201 & 0.9503 & 0.9351 & 0.8032 \\
\hline $25.0 \%$ & 0.7441 & 0.6825 & 0.5129 & 0.9453 & 0.9024 & 0.6883 & 0.9420 & 0.8794 & 0.5434 & 0.9163 & 0.8689 & 0.6917 & 0.9462 & 0.9414 & 0.5610 & 0.9564 & 0.9448 & 0.8441 \\
\hline $50.0 \%$ & 0.8654 & 0.8229 & 0.6700 & 0.9483 & 0.9076 & 0.7205 & 0.9456 & 0.8886 & 0.7624 & 0.9224 & 0.8773 & 0.7067 & 0.9514 & 0.9457 & 0.6468 & 0.9591 & 0.9497 & 0.8625 \\
\hline $75.0 \%$ & 0.9192 & 0.9028 & 0.8141 & 0.9511 & 0.9121 & 0.7405 & 0.9490 & 0.8979 & 0.7871 & 0.9284 & 0.8845 & 0.7227 & 0.9559 & 0.9497 & 0.7460 & 0.9619 & 0.9541 & 0.8777 \\
\hline $97.5 \%$ & 0.9757 & 0.9779 & 0.9471 & 0.9565 & 0.9201 & 0.7707 & 0.9550 & 0.9131 & 0.8196 & 0.9384 & 0.8968 & 0.7510 & 0.9636 & 0.9576 & 0.8299 & 0.9671 & 0.9618 & 0.9040 \\
\hline
\end{tabular}


Table 5.3 Correlation of NHF with other HRV parameters that measures vagal activity NHF -- SDSD

\begin{tabular}{|c|c|c|c|c|c|c|c|c|c|c|c|c|c|c|c|c|c|c|}
\hline Stat & \multicolumn{3}{|c|}{ individual } & \multicolumn{3}{|c|}{ Bootstrap-All Infants } & \multicolumn{3}{|c|}{ Bootstrap-ALTE } & \multicolumn{3}{|c|}{ Bootstrap-Preterm } & \multicolumn{3}{|c|}{ Bootstrap-Siblings } & \multicolumn{3}{|c|}{ Bootstrap-Healthy } \\
\hline & QS & REM & AWK & QS & REM & AWK & QS & REM & AWK & QS & REM & AWK & QS & REM & AWK & QS & REM & AWK \\
\hline $2.5 \%$ & -0.5195 & -0.5844 & -0.4643 & 0.3452 & 0.2920 & 0.2061 & 0.4458 & 0.4654 & 0.1428 & 0.2465 & 0.1806 & 0.2323 & 0.1639 & 0.3287 & -0.0008 & 0.3002 & 0.2458 & 0.1664 \\
\hline $25.0 \%$ & -0.2454 & -0.0224 & 0.0232 & 0.3778 & 0.3257 & 0.2631 & 0.4767 & 0.5300 & 0.2254 & 0.2892 & 0.2247 & 0.2716 & 0.2186 & 0.3949 & 0.1126 & 0.3437 & 0.3368 & 0.2675 \\
\hline $50.0 \%$ & 0.0592 & 0.2415 & 0.2454 & 0.3948 & 0.3433 & 0.2877 & 0.4952 & 0.5583 & 0.3333 & 0.3112 & 0.2457 & 0.2923 & 0.2497 & 0.4262 & 0.1771 & 0.3656 & 0.3741 & 0.3167 \\
\hline $75.0 \%$ & 0.2769 & 0.4234 & 0.4290 & 0.4139 & 0.3614 & 0.3126 & 0.5142 & 0.5870 & 0.3837 & 0.3354 & 0.2689 & 0.3165 & 0.2814 & 0.4580 & 0.2487 & 0.3885 & 0.4105 & 0.3653 \\
\hline $97.5 \%$ & 0.8093 & 0.7355 & 0.7271 & 0.4468 & 0.3957 & 0.3519 & 0.5474 & 0.6415 & 0.4492 & 0.3799 & 0.3096 & 0.3571 & 0.3467 & 0.5131 & 0.3786 & 0.4220 & 0.4794 & 0.4591 \\
\hline std & 0.3558 & 0.3442 & 0.2931 & 0.0261 & 0.0261 & 0.0364 & 0.0258 & 0.0447 & 0.0902 & 0.0340 & 0.0331 & 0.0320 & 0.0463 & 0.0466 & 0.0976 & 0.0313 & 0.0569 & 0.0736 \\
\hline
\end{tabular}

NHF -- IQRSD

\begin{tabular}{|c|c|c|c|c|c|c|c|c|c|c|c|c|c|c|c|c|c|c|}
\hline \multirow[t]{2}{*}{ Stat } & \multicolumn{3}{|c|}{ individual } & \multicolumn{3}{|c|}{ Bootstrap-All Infants } & \multicolumn{3}{|c|}{ Bootstrap-ALTE } & \multicolumn{3}{|c|}{ Bootstrap-Preterm } & \multicolumn{3}{|c|}{ Bootstrap-Siblings } & \multicolumn{3}{|c|}{ Bootstrap-Healthy } \\
\hline & QS & REM & AWK & QS & REM & AWK & QS & REM & AWK & QS & REM & AWK & QS & REM & AWK & QS & REM & AWK \\
\hline $2.5 \%$ & -0.4782 & -0.5591 & -0.5266 & 0.4426 & 0.3317 & 0.1631 & 0.5174 & 0.4751 & 0.1982 & 0.3596 & 0.2544 & 0.1768 & 0.2966 & 0.3732 & -0.0746 & 0.3689 & 0.2475 & 0.1432 \\
\hline $25.0 \%$ & -0.0582 & -0.0102 & -0.0866 & 0.4740 & 0.3662 & 0.2063 & 0.5509 & 0.5401 & 0.2697 & 0.4075 & 0.3007 & 0.2193 & 0.3510 & 0.4313 & 0.0122 & 0.4110 & 0.3231 & 0.2559 \\
\hline $50.0 \%$ & 0.2020 & 0.2235 & 0.1734 & 0.4900 & 0.3834 & 0.2300 & 0.5682 & 0.5685 & 0.3174 & 0.4306 & 0.3179 & 0.2440 & 0.3806 & 0.4652 & 0.0611 & 0.4334 & 0.3608 & 0.3135 \\
\hline $75.0 \%$ & 0.4102 & 0.4293 & 0.3384 & 0.5068 & 0.3993 & 0.2545 & 0.5850 & 0.5988 & 0.3544 & 0.4568 & 0.3365 & 0.2701 & 0.4103 & 0.4978 & 0.1099 & 0.4549 & 0.3980 & 0.3843 \\
\hline $97.5 \%$ & 0.7479 & 0.7547 & 0.7168 & 0.5375 & 0.4284 & 0.2965 & 0.6143 & 0.6493 & 0.4242 & 0.5026 & 0.3720 & 0.3150 & 0.4670 & 0.5511 & 0.2160 & 0.4958 & 0.4580 & 0.4832 \\
\hline std & 0.3484 & 0.3449 & 0.3311 & 0.0245 & 0.0246 & 0.0345 & 0.0253 & 0.0464 & 0.0605 & 0.0366 & 0.0298 & 0.0361 & 0.0441 & 0.0475 & 0.0734 & 0.0319 & 0.0540 & 0.0891 \\
\hline
\end{tabular}

NHF -- HF

\begin{tabular}{|c|c|c|c|c|c|c|c|c|c|c|c|c|c|c|c|c|c|c|}
\hline \multirow[t]{2}{*}{ Stat } & \multicolumn{3}{|c|}{ individual } & \multicolumn{3}{|c|}{ Bootstrap-All Infants } & \multicolumn{3}{|c|}{ Bootstrap-ALTE } & \multicolumn{3}{|c|}{ Bootstrap-Preterm } & \multicolumn{3}{|c|}{ Bootstrap-Siblings } & \multicolumn{3}{|c|}{ Bootstrap-Healthy } \\
\hline & QS & REM & AWK & QS & REM & AWK & QS & REM & AWK & QS & REM & AWK & QS & REM & AWK & QS & REM & AWK \\
\hline $2.5 \%$ & -0.5783 & -0.4084 & .3745 & 0.3269 & 0.2712 & 0.0842 & 0.4294 & 0.4565 & 0.2013 & 0.1900 & 0.1440 & 0.1202 & 0.1868 & 0.3348 & -0.0196 & 0.3677 & 0.2834 & 0.1310 \\
\hline $25.0 \%$ & -0.2303 & 0.1220 & 004 & 0.3679 & 0.3054 & 0.1714 & 0.4657 & 0.5111 & 0.2638 & 0.2374 & 0.1913 & 0.1675 & 0.2446 & 0.3986 & 0.0394 & 0.4052 & 651 & 0.2188 \\
\hline $50.0 \%$ & 0.1719 & 3519 & & 0.3863 & .3244 & 2005 & .4874 & 0.5442 & 0.2959 & 9 & 0.2140 & & 0.2773 & & & & & 0.2627 \\
\hline $75.0 \%$ & 0.3630 & 5761 & 4116 & 0.4082 & 0.3432 & 0.2302 & 0.5070 & 0.5729 & 0.3271 & 0.2955 & & 0.2363 & 0.3124 & & 0.1844 & 0.4496 & & 0.3094 \\
\hline $97.5 \%$ & & & & & & & & & & & & & & & & & & 0.4011 \\
\hline std & 0.3761 & 0.3286 & 0.2956 & 0.0299 & 0.0276 & 0.0508 & 0.0298 & 0.0439 & 0.0484 & 0.0447 & 0.0321 & 0.0457 & 0.0493 & 0.0498 & 0.0944 & 0.0316 & 0.0663 & 0.0687 \\
\hline
\end{tabular}

NHF -- HFW

\begin{tabular}{|c|c|c|c|c|c|c|c|c|c|c|c|c|c|c|c|c|c|c|}
\hline \multirow[t]{2}{*}{ Stat } & \multicolumn{3}{|c|}{ individual } & \multicolumn{3}{|c|}{ Bootstrap-All Infants } & \multicolumn{3}{|c|}{ Bootstrap-ALTE } & \multicolumn{3}{|c|}{ Bootstrap-Preterm } & \multicolumn{3}{|c|}{ Bootstrap-Siblings } & \multicolumn{3}{|c|}{ Bootstrap-Healthy } \\
\hline & QS & REM & AWK & QS & REM & $\mathrm{AWK}$ & QS & REM & AWK & QS & REM & AWK & QS & REM & AWK & QS & REM & AWK \\
\hline $2.5 \%$ & -0.5562 & -0.5591 & -0.4444 & 0.3283 & 0.2295 & 0.0424 & 0.4232 & 0.4219 & 0.0591 & 0.1529 & 0.0989 & 0.0364 & 0.1945 & 0.3355 & -0.0694 & 0.3929 & 0.1599 & 0.0368 \\
\hline $25.0 \%$ & -0.1851 & 0.0449 & -0.1299 & 0.3629 & 0.2627 & 0.0825 & 0.4612 & 0.48 & 0.1365 & 0.2035 & 0.1408 & 0.0739 & 0.2555 & 0.3905 & 0.0036 & 0.4316 & 0.2717 & 0.1152 \\
\hline $50.0 \%$ & 0.0921 & 0.2401 & 0.0535 & 0.3846 & 0.2776 & 0.1063 & 0.4819 & 0.5163 & 0.1759 & 0.2312 & 0.1622 & 0.0946 & 0.2907 & 0.4224 & 0.0436 & 0.4529 & 0.3145 & 0.1737 \\
\hline $75.0 \%$ & 0.3263 & 0.4595 & 0.2503 & 0.4032 & 0.2950 & 0.1295 & 0.5032 & 0.5484 & 0.2233 & 0.2628 & 0.1836 & 0.1185 & 0.3263 & 0.4543 & 0.0918 & 0.4746 & 0.3457 & 0.2260 \\
\hline $97.5 \%$ & 0.8264 & 0.8098 & 0.7000 & 0.4386 & 0.3274 & 0.1760 & 0.5415 & 0.6067 & 0.3007 & 0.3230 & 0.2235 & 0.1727 & 0.3962 & 0.5109 & 0.1975 & 0.5131 & 0.4032 & 0.3307 \\
\hline std & 0.3638 & 0.3405 & 0.2945 & 0.0287 & 0.0245 & 0.0341 & 0.0309 & 0.0478 & 0.0637 & 0.0450 & 0.0317 & 0.0340 & 0.0514 & 0.0457 & 0.0673 & 0.0311 & 0.0603 & 0.0776 \\
\hline
\end{tabular}


Table 5.4 Correlation of frequency domain HRV parameters

Total Power -- SDNN

\begin{tabular}{|c|c|c|c|c|c|c|c|c|c|c|c|c|c|c|c|c|c|c|}
\hline \multirow[t]{2}{*}{ Stat } & \multicolumn{3}{|c|}{ individual } & \multicolumn{3}{|c|}{ Bootstrap-All Infants } & \multicolumn{3}{|c|}{ Bootstrap-ALTE } & \multicolumn{3}{|c|}{ Bootstrap-Preterm } & \multicolumn{3}{|c|}{ Bootstrap-Siblings } & \multicolumn{3}{|c|}{ Bootstrap-Healthy } \\
\hline & QS & REM & AWK & QS & REM & AWK & QS & REM & AWK & QS & REM & AWK & QS & REM & AWK & QS & REM & AWK \\
\hline $2.5 \%$ & 0.1827 & 0.1404 & 0.0328 & 0.7599 & 0.7383 & 0.4870 & 0.7327 & 0.5604 & 0.4937 & 0.7509 & 0.7265 & 0.4675 & 0.7123 & 0.7427 & 0.5019 & 0.7313 & 0.7278 & 0.4878 \\
\hline $25.0 \%$ & 0.6408 & 0.4498 & 0.3918 & 0.7905 & 0.7625 & 0.5297 & 0.7598 & 0.6091 & 0.5875 & 0.7930 & 0.7572 & 0.5194 & 0.7554 & 0.7806 & 0.5762 & 0.7648 & 0.7637 & 0.5627 \\
\hline $50.0 \%$ & 0.7652 & 0.5990 & 0.5532 & 0.8026 & 0.7743 & 0.5668 & 0.7752 & 0.6327 & 0.6376 & 0.8124 & 0.7734 & 0.5466 & 0.7786 & 0.7986 & 0.6214 & 0.7807 & 0.7846 & 0.6025 \\
\hline $75.0 \%$ & 0.8391 & 0.7276 & 0.7001 & 0.8165 & 0.7860 & 0.6385 & 0.7883 & 0.6582 & 0.9437 & 0.8317 & 0.7897 & 0.5735 & 0.7990 & 0.8137 & 0.6948 & 0.7966 & 0.8030 & 0.6412 \\
\hline $97.5 \%$ & 0.9606 & 0.8769 & 0.9750 & 0.8417 & 0.8093 & 0.8775 & 0.8104 & 0.6971 & 0.9587 & 0.8609 & 0.8211 & 0.6437 & 0.8349 & 0.8418 & 0.8058 & 0.8218 & 0.8347 & 0.6954 \\
\hline std & 0.1967 & 0.2021 & 0.2340 & 0.0209 & 0.0179 & 0.1297 & 0.0200 & 0.0362 & 0.1691 & 0.0289 & 0.0237 & 0.0428 & 0.0317 & 0.0249 & 0.0812 & 0.0233 & 0.0279 & 0.0556 \\
\hline & & & & & & & & & & & & & & & & & $\begin{array}{c}0.0328 \\
\min \end{array}$ & $\begin{array}{c}0.975 \\
\max \end{array}$ \\
\hline
\end{tabular}

Total Power -- LF

\begin{tabular}{|c|c|c|c|c|c|c|c|c|c|c|c|c|c|c|c|c|c|c|}
\hline \multirow[t]{2}{*}{ Stat } & \multicolumn{3}{|c|}{ individual } & \multicolumn{3}{|c|}{ Bootstrap-All Infants } & \multicolumn{3}{|c|}{ Bootstrap-ALTE } & \multicolumn{3}{|c|}{ Bootstrap-Preterm } & \multicolumn{3}{|c|}{ Bootstrap-Siblings } & \multicolumn{3}{|c|}{ Bootstrap-Healthy } \\
\hline & QS & REM & AWK & $\mathrm{QS}$ & REM & AWK & QS & REM & AWK & QS & REM & AWK & QS & REM & AWK & QS & REM & AW K \\
\hline $2.5 \%$ & 0.7645 & 0.9718 & 0.9447 & 0.9553 & 0.9950 & 0.9937 & 0.9081 & 0.9853 & 0.9836 & 0.9814 & 0.9972 & 0.9936 & 0.9593 & 0.9947 & 0.9930 & 0.9301 & 0.9898 & 0.98 \\
\hline $25.0 \%$ & 0.9678 & 0.9936 & 0.9913 & 0.9650 & 0.9958 & 0.9958 & 0.9243 & 0.9888 & 0.9892 & 0.9878 & 0.9977 & 0.9961 & 0.9757 & 0.9962 & 0.9962 & 0.9420 & 0.992 & $0.9 \varsigma$ \\
\hline $50.0 \%$ & 0.9941 & 0.9974 & 0.9972 & 0.9701 & 0.9962 & 0.9973 & 0.9318 & 0.9903 & 0.9940 & 0.9908 & 0.9979 & 0.9973 & 0.9822 & 0.9968 & 0.9976 & 0.9478 & 0.9936 & 0.9942 \\
\hline $75.0 \%$ & 0.9984 & 0.9987 & 0.9991 & 0.9745 & 0.9965 & 0.9984 & 0.9384 & 0.9918 & 1.0000 & 0.9932 & 0.9981 & 0.9984 & 0.9863 & 0.9973 & 0.9989 & 0.9534 & 0.9946 & 0.9956 \\
\hline $97.5 \%$ & 0.9998 & 0.9998 & 1.0000 & 0.9809 & 0.9970 & 0.9999 & 0.9495 & 0.9939 & 1.0000 & 0.9959 & 0.9985 & 0.9990 & 0.9912 & 0.9979 & 0.9997 & 0.9617 & 0.9960 & 0.9974 \\
\hline std & 0.0919 & 0.0327 & 0.0150 & 0.0068 & 0.0005 & 0.0019 & 0.0107 & 0.0022 & 0.0055 & 0.0038 & 0.0003 & 0.0015 & 0.0084 & 0.0008 & 0.0018 & 0.0083 & 0.0016 & 0.0021 \\
\hline
\end{tabular}

$\min \max$

LF -- SDNN

\begin{tabular}{|c|c|c|c|c|c|c|c|c|c|c|c|c|c|c|c|c|c|c|}
\hline \multirow[t]{2}{*}{ Stat } & \multicolumn{3}{|c|}{ individual } & \multicolumn{3}{|c|}{ Bootstrap-All Infants } & \multicolumn{3}{|c|}{ Bootstrap-ALTE } & \multicolumn{3}{|c|}{ Bootstrap-Preterm } & \multicolumn{3}{|c|}{ Bootstrap-Siblings } & \multicolumn{3}{|c|}{ Bootstrap-Healthy } \\
\hline & QS & REM & AWK & QS & REM & AWK & QS & REM & AWK & QS & REM & AWK & QS & REM & AWK & QS & REM & AWK \\
\hline $2.5 \%$ & 0.0973 & 0.1284 & 0.0302 & 0.7025 & 0.7206 & 0.4742 & 0.6455 & 0.5491 & 0.4811 & 0.7274 & 0.7089 & 0.4486 & 0.6713 & 0.7212 & 0.4987 & 0.6519 & 0.6940 & 0.4738 \\
\hline $25.0 \%$ & 0.5838 & 0.4365 & 0.3421 & 0.7341 & 0.7465 & 0.5228 & 0.6805 & 0.6012 & 0.5805 & 0.7693 & 0.7441 & 0.5071 & 0.7181 & 0.7630 & 0.5749 & 0.6836 & 0.7319 & 0.5467 \\
\hline $50.0 \%$ & 0.7335 & 0.5932 & 0.5372 & 0.7506 & 0.7597 & 0.5571 & 0.6979 & 0.6250 & 0.6278 & 0.7902 & 0.7615 & 0.5353 & 0.7417 & 0.7829 & 0.6174 & 0.7013 & 0.7540 & 0.5877 \\
\hline $75.0 \%$ & 0.8057 & 0.7140 & 0.6970 & 0.7652 & 0.7720 & 0.6358 & 0.7144 & 0.6512 & 0.9429 & 0.8115 & 0.7782 & 0.5632 & 0.7665 & 0.7990 & 0.6948 & 0.7174 & 0.7738 & 0.6257 \\
\hline $97.5 \%$ & 0.9379 & 0.8763 & 0.9780 & 0.7956 & 0.7963 & 0.8752 & 0.7486 & 0.6947 & 0.9582 & 0.8423 & 0.8119 & 0.6392 & 0.8078 & 0.8319 & 0.8187 & 0.7483 & 0.8064 & 0.6851 \\
\hline std & 0.2118 & 0.2035 & 0.2396 & 0.0235 & 0.0194 & 0.1324 & 0.0256 & 0.0373 & 0.1729 & 0.0308 & 0.0253 & 0.0463 & 0.0351 & 0.0270 & 0.0855 & 0.0245 & 0.0297 & 0.0565 \\
\hline
\end{tabular}

$\min \max$ 
Table 5.4 Correlation of frequency domain HRV parameters (cont.)

Total Power -- LF

\begin{tabular}{|c|c|c|c|c|c|c|c|c|c|c|c|c|c|c|c|c|c|c|}
\hline Stat & \multicolumn{3}{|c|}{ individual } & \multicolumn{3}{|c|}{ Bootstrap-All Infants } & \multicolumn{3}{|c|}{ Bootstrap-ALTE } & \multicolumn{3}{|c|}{ Bootstrap-Preterm } & \multicolumn{3}{|c|}{ Boots trap-Siblings } & \multicolumn{3}{|c|}{ Bootstrap-Healthy } \\
\hline & QS & REM & $\mathrm{AWK}$ & $\mathrm{QS}$ & REM & AWK & $\mathrm{QS}$ & REM & AWK & QS & REM & AWK & QS & REM & $\mathrm{AWK}$ & & REM & AWK \\
\hline 2.5 & 0.066 & & & & & & & & & & & & & & & & & \\
\hline 25.0 & & & & & & & & & & & & & & & & & & \\
\hline & & & & & & & & & & & & & & & & & & \\
\hline & & & & & & & & & & & & & & & & & & \\
\hline $97.5^{c}$ & 654 & & & 0.7652 & 0.8011 & & & & & & & & & & & & & \\
\hline stc & 0.2244 & 0.2145 & 21 & 0.0285 & 0.0206 & 0.24 & .0306 & & 0.2694 & 0.0344 & 0.0235 & 0.0786 & 0.0485 & 0.0277 & & 0.0382 & 0.0396 & 0.051 \\
\hline
\end{tabular}

0.01830 .9863

HF -- SDNN

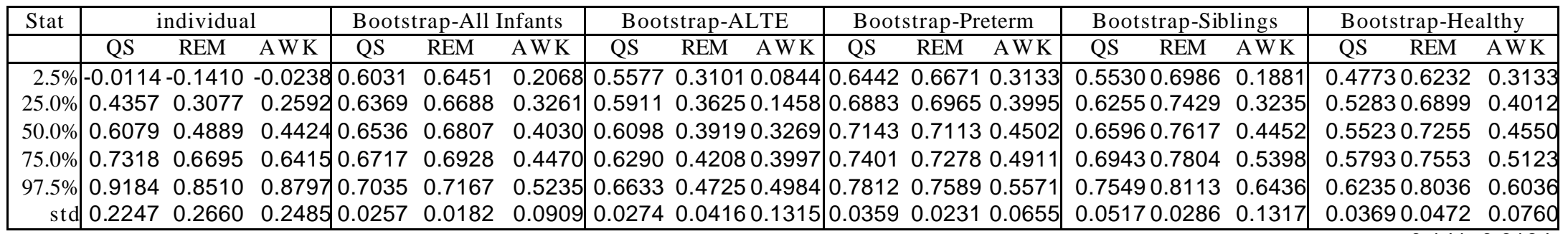

Total Power -- RMSSD

\begin{tabular}{|c|c|c|c|c|c|c|c|c|c|c|c|c|c|c|c|c|c|c|}
\hline Stat & \multicolumn{3}{|c|}{ individual } & \multicolumn{3}{|c|}{ Bootstrap-All Infants } & \multicolumn{3}{|c|}{ Bootstrap-ALTE } & \multicolumn{3}{|c|}{ Bootstrap-Preterm } & \multicolumn{3}{|c|}{ Bootstrap-Siblings } & \multicolumn{3}{|c|}{ Bootstrap-Healthy } \\
\hline & QS & REM & AWK & QS & REM & AWK & $\overline{Q S}$ & REM & AWK & QS & REM & AWK & & REM & $\mathrm{AWK}$ & & REM & AWK \\
\hline $2.5 \%$ & 0.0743 & -0.1331 & -0.1548 & 0.6159 & 0.6279 & 0.3517 & 0.6186 & 0.3963 & $\overline{0.4076}$ & 0.5951 & 0.5996 & 0.3134 & 0.5976 & 0.7387 & 0.4777 & 0.5074 & 0.5737 & 0.3751 \\
\hline $25.0 \%$ & 0.4281 & 0.3522 & 0.2950 & 0.6490 & 0.6574 & 0.4100 & 0.6486 & 0.4553 & 0.4961 & 0.6371 & 0.6371 & 630 & 0.6478 & 0.7740 & 0.5729 & 0.551 & 6304 & 0.4573 \\
\hline 50.09 & 0.6093 & 0.5457 & 0.5058 & 0.6657 & 0.6714 & 0.4317 & 0.6675 & 0.4882 & 0.5469 & 0.6579 & 0.6550 & 0.4006 & 0.6738 & 0.7925 & 0.6142 & 0.5738 & 0.6557 & 0.5043 \\
\hline $75.0 \%$ & 0.6915 & 0.6782 & 0.6906 & 0.6816 & 0.6839 & 0.4819 & 0.6854 & 0.5219 & 0.6077 & 0.6788 & 0.6703 & 0.4429 & 0.6983 & 0.8075 & 0.6594 & 0.5959 & 0.6852 & 0.5481 \\
\hline $97.5 \%$ & 0.8791 & 0.9091 & 0.9448 & 0.7097 & 0.7084 & 0.5500 & 0.7198 & 0.5812 & 0.6421 & 0.7140 & 0.7006 & 0.5139 & 0.7375 & 0.8340 & 0.7433 & 0.6330 & 0.7315 & 0.6229 \\
\hline std & 0.2023 & 0.2564 & 0.2868 & 0.0243 & 0.0199 & 0.0510 & 0.0259 & 0.0473 & 0.0669 & 0.0306 & 0.0250 & 0.0537 & 0.0365 & 0.0245 & 0.0658 & 0.0320 & 0.0411 & 0.0639 \\
\hline
\end{tabular}

LF -- RMSSD

\begin{tabular}{|c|c|c|c|c|c|c|c|c|c|c|c|c|c|c|c|c|c|c|}
\hline Stat & \multicolumn{3}{|c|}{ individual } & \multicolumn{3}{|c|}{ Bootstrap-All Infants } & \multicolumn{3}{|c|}{ Bootstrap-ALTE } & \multicolumn{3}{|c|}{ Bootstrap-Preterm } & \multicolumn{3}{|c|}{ Bootstrap-Siblings } & \multicolumn{3}{|c|}{ Bootstrap-Healthy } \\
\hline & $\mathrm{QS}$ & REM & AWK & QS & REM & AWK & $\mathrm{QS}$ & REM & AWK & QS & REM & AWK & QS & REM & AWK & $\mathrm{QS}$ & REM & AWK \\
\hline $2.5 \%$ & -0.0559 & -0.2795 & -0.2475 & 0.4440 & 0.5712 & 0.3101 & 0.3163 & 0.2891 & 0.3050 & 0.5009 & 0.5582 & 0.2722 & 0.4235 & 0.6863 & 0.4216 & 0.2484 & 0.4947 & 0.3008 \\
\hline $25.0 \%$ & 0.2547 & 0.3035 & 0.1914 & 0.4824 & 0.6044 & 0.3752 & 0.3640 & 0.3545 & 0.4099 & 0.5510 & 0.5966 & 0.3223 & 0.5043 & 0.7292 & 0.5393 & 0.2982 & .5602 & 0.3812 \\
\hline $50.0 \%$ & 0.4285 & 0.4952 & 0.4476 & 0.5013 & 0.6205 & 0.4043 & 0.3898 & 0.390 & 0.4673 & 0.5758 & 0.6167 & 0.3548 & 0.5450 & 0.7523 & 0.5871 & 0.3218 & 30.5884 & 0.4291 \\
\hline $75.0 \%$ & 0.6319 & 0.6518 & 0.6616 & 0.5216 & 0.6348 & 0.4276 & 0.4185 & 0.4249 & 0.6024 & 0.5989 & 0.6336 & 0.3931 & 0.5843 & 0.7710 & 0.6309 & 0.3452 & 0.6193 & 0.4822 \\
\hline $97.5 \%$ & 0.8436 & 0.8872 & 0.9399 & 0.5540 & 0.6616 & 0.4958 & 0.4701 & 0.4895 & 0.6387 & 0.6341 & 0.6657 & 0.4618 & 0.6411 & 0.8035 & 0.7122 & 0.3878 & 30.6727 & 0.5687 \\
\hline std & 0.2560 & 0.2851 & 0.3099 & 0.0284 & 0.0226 & 0.0445 & 0.0389 & 0.0514 & 0.1037 & 0.0355 & 0.0277 & 0.0491 & 0.0565 & 0.0301 & 0.0721 & 0.0351 & 0.0464 & 0.0700 \\
\hline
\end{tabular}


Table 5.4 Correlation of frequency domain HRV parameters (cont.)

\begin{tabular}{|c|c|c|c|c|c|c|c|c|c|c|c|c|c|c|c|c|c|c|}
\hline Stat & \multicolumn{3}{|c|}{ individual } & \multicolumn{3}{|c|}{ Bootstrap-All Infants } & \multicolumn{3}{|c|}{ Bootstrap-ALTE } & \multicolumn{3}{|c|}{ Bootstrap-Preterm } & \multicolumn{3}{|c|}{ Bootstrap-Siblings } & \multicolumn{3}{|c|}{ Bootstrap-Healthy } \\
\hline & $\mathrm{QS}$ & REM & AWK & QS & REM & AWK & QS & REM & AWK & QS & REM & AWK & QS & REM & AWK & QS & REM & AWK \\
\hline $2.5 \%$ & -0.1496 & 0.0515 & -0.0437 & 0.4359 & 0.6514 & 0.0462 & 0.3313 & 0.3916 & 0.0113 & 0.6047 & 0.6949 & 0.3752 & 0.4556 & 0.7228 & 0.3593 & 0.2097 & 0.5300 & 0.4188 \\
\hline $25.0 \%$ & 0.3250 & 0.3975 & 0.3512 & 0.4866 & 0.6878 & 0.4192 & 0.3955 & 0.4571 & 0.0207 & 0.6746 & 0.7291 & 0.4908 & 0.5690 & 0.7792 & 0.5458 & 0.2628 & 0.6013 & 0.4922 \\
\hline $50.0 \%$ & 0.5509 & 0.6881 & 0.5796 & 0.5156 & 0.7067 & 0.5275 & 0.4327 & 0.4864 & 0.4258 & 0.7122 & 0.7483 & 0.5665 & 0.6238 & 0.8038 & 0.6630 & 0.2902 & 0.6367 & 0.5356 \\
\hline $75.0 \%$ & 0.8188 & 0.7939 & 0.7749 & 0.5446 & 0.7221 & 0.6094 & 0.4728 & 0.5208 & 0.4866 & 0.7496 & 0.7668 & 0.6319 & 0.6785 & 0.8270 & 0.7791 & 0.3186 & 0.6700 & 0.5697 \\
\hline $97.5 \%$ & 0.9390 & 0.9532 & 0.9790 & 0.6012 & 0.7507 & 0.8478 & 0.5377 & 0.5725 & 0.5668 & 0.8160 & 0.8043 & 0.7077 & 0.7645 & 0.8682 & 0.9815 & 0.3692 & 0.7334 & 0.6367 \\
\hline std & 0.2988 & 0.2635 & 0.2883 & 0.0424 & 0.0251 & 0.2315 & 0.0540 & 0.0469 & 0.2195 & 0.0532 & 0.0281 & 0.0936 & 0.0795 & 0.0361 & 0.1673 & 0.0405 & 0.0514 & 0.0564 \\
\hline
\end{tabular}

$\mathrm{HF}-\mathrm{LF} / \mathrm{HF}$

\begin{tabular}{|c|c|c|c|c|c|c|c|c|c|c|c|c|c|c|c|c|c|c|}
\hline Stat & \multicolumn{2}{|c|}{ individual } & \multicolumn{3}{|c|}{ Bootstrap-All Infants } & \multicolumn{3}{|c|}{ Bootstrap-ALTE } & \multicolumn{3}{|c|}{ Bootstrap-Preterm } & \multicolumn{3}{|c|}{ Bootstrap-Siblings } & \multicolumn{4}{|c|}{ Bootstrap-Healthy } \\
\hline & REM & AWK & QS & REM & AWK & QS & REM & AWK & QS & REM & AWK & QS & REM & $\mathrm{AWF}$ & & QS & REM & AWK \\
\hline $.5 \%$ & $3-0.8109$ & -0.6220 & 0.28 & 0.3447 & -0.2627 & -0.335 & $\overline{0504}$ & -0.3661 & -0.26 & 0.2853 & -0.229 & -0.2726 & -0.3528 & -0.326 & & -0.3362 & -0.5252 & $\overline{3828}$ \\
\hline $25.0 \%$ & & & -( & & & & & & -( & & & 29 & & & & 26 & & \\
\hline $50.0 \%$ & $-0.0966-0.3$ & -0.2366 & -0.243 & -0.3137 & -0.1845 & -0.299 & -0.4 & -0.2875 & -0.20 & -0.2 & -0.1 & -0.2144 & -0.3164 & $-0.18 \varepsilon$ & & -0.3006 & -0.4533 & -0.3029 \\
\hline $75.0 \%$ & $0.2186-0.1$ & -0.0262 & -0.231 & -0.3006 & -0.1090 & -0.289 & -0.429 & -0.0227 & -0.186 & -0.22 & & -0.1953 & -0.3041 & -0.1 & & -0.2893 & -0.4258 & -0.2791 \\
\hline $97.5 \%$ & 0.69990 .4174 & 0.4623 & -0.20 & & -0.0194 & 1007 & & & -0.1 & & & -0.1600 & -0.2 & & & -0.2689 & & \\
\hline std & 0.36460 .2986 & 0.2745 & 0.0190 & 0.0181 & 0.0788 & 0.0159 & 0.0284 & 0.1416 & 0.0270 & 0.0235 & 0.038 & 0.0284 & 0.0181 & 0.07 & & 0.0174 & 0.0393 & 0.0377 \\
\hline
\end{tabular}

$\mathrm{LF}-\mathrm{LF} / \mathrm{HF}$

\begin{tabular}{|c|c|c|c|c|c|c|c|c|c|c|c|c|c|c|c|c|c|c|}
\hline Stat & \multicolumn{3}{|c|}{ individual } & \multicolumn{3}{|c|}{ Bootstrap-All Infants } & \multicolumn{3}{|c|}{ Bootstrap-ALTE } & \multicolumn{3}{|c|}{ Bootstrap-Preterm } & \multicolumn{3}{|c|}{ Bootstrap-Siblings } & \multicolumn{3}{|c|}{ Bootstrap-Healthy } \\
\hline & QS & REM & AWK & QS & REM & AWK & $\mathrm{QS}$ & REM & AWK & QS & REM & AWK & $\mathrm{QS}$ & REM & AWK & QS & REM & AWK \\
\hline $2.5 \%$ & -0.0249 & -0.1965 & -0.1330 & 0.1047 & 0.0582 & 0.1655 & 0.2556 & 0.1359 & 0.1338 & 0.0331 & 0.0784 & 0.2152 & 0.2173 & -0.0845 & 0.0177 & 0.3881 & 0.0115 & 0.0919 \\
\hline $25.0 \%$ & 0.5262 & 0.2551 & 0.3254 & 0.1454 & 0.0902 & 0.2523 & 0.3335 & 0.2024 & 0.1938 & 0.0652 & 0.1218 & 0.3035 & 0.2894 & -0.0371 & 0.2124 & 0.4284 & 0.0781 & 0.1749 \\
\hline $50.0 \%$ & 0.7242 & 0.4520 & 0.5139 & 0.1700 & 0.1074 & 0.3247 & 0.3679 & 0.2362 & 0.2465 & 0.0822 & 0.1455 & 0.3618 & 0.3308 & -0.0034 & 0.3262 & 0.4515 & 0.1186 & 0.2285 \\
\hline $75.0 \%$ & 0.8521 & 0.6592 & 0.7007 & 0.1950 & 0.1268 & 0.4311 & 0.4012 & 0.2717 & 0.9973 & 0.1032 & 0.1740 & 0.4303 & 0.3775 & 0.0282 & 0.4468 & 0.4740 & 0.1641 & 0.2930 \\
\hline $97.5 \%$ & 0.9793 & 0.9068 & 0.9498 & 0.2451 & 0.1680 & 0.9933 & 0.4530 & 0.3391 & 0.9985 & 0.1454 & 0.2256 & 0.5500 & 0.4723 & 0.0864 & 0.6996 & 0.5101 & 0.2366 & 0.4181 \\
\hline std & 0.2651 & 0.3045 & 0.2870 & 0.0369 & 0.0278 & 0.2836 & 0.0502 & 0.0518 & 0.3755 & 0.0283 & 0.0378 & 0.0882 & 0.0652 & 0.0459 & 0.1774 & 0.0317 & 0.0591 & 0.0850 \\
\hline
\end{tabular}

LF/HF - Total Power

\begin{tabular}{|c|c|c|c|c|c|c|c|c|c|c|c|c|c|c|c|c|c|c|}
\hline Stat & \multicolumn{3}{|c|}{ individual } & \multicolumn{3}{|c|}{ Bootstrap-All Infants } & \multicolumn{3}{|c|}{ Bootstrap-ALTE } & \multicolumn{3}{|c|}{ Bootstrap-Preterm } & \multicolumn{3}{|c|}{ Bootstrap-Siblings } & \multicolumn{3}{|c|}{ Bootstrap-Healthy } \\
\hline & QS & REM & AWK & QS & REM & AWK & QS & REM & AWK & QS & REM & AWK & QS & REM & AWK & QS & REM & AWK \\
\hline $2.5 \%$ & -0.2794 & -0.3434 & -0.1666 & 0.0164 & 0.0111 & 0.1316 & 0.0595 & 0.0526 & 0.0630 & -0.0163 & 0.0501 & 0.1910 & 0.1349 & -0.1253 & 0.0062 & 0.2105 & -0.0768 & 0.0423 \\
\hline $25.0 \%$ & 0.3006 & 0.1545 & 0.2502 & 0.0482 & 0.0422 & 0.2140 & 0.1280 & 0.1113 & 0.1276 & 0.0140 & 0.0899 & 0.2782 & 0.1898 & -0.0755 & 0.1905 & 0.2591 & -0.0004 & 0.1333 \\
\hline $50.0 \%$ & 0.6118 & 0.3622 & 0.4431 & 0.0702 & 0.0597 & 0.2824 & 1615 & 0.1459 & 0.1801 & 0.0317 & 0.1134 & 0.3337 & 0.2245 & -0.0490 & 0.2819 & 0.2862 & 0.0422 & 0.1859 \\
\hline $75.0 \%$ & 0.8053 & 0.6073 & 0.6512 & 0.0930 & 0.0782 & 0.3757 & 0.1899 & 0.1793 & 0.9970 & 0.0494 & 0.1410 & 0.3969 & 0.2599 & -0.0191 & 0.3961 & 0.3114 & 862 & 0.2416 \\
\hline $97.5 \%$ & 0.9588 & 0.8872 & 0.9388 & 0.1386 & 0.1134 & 0.9921 & 0.2300 & 0.2452 & 0.9983 & 0.0953 & 0.1899 & 0.5030 & 0.3356 & 0.0445 & 0.6751 & 0.3615 & 0.1573 & 0.3641 \\
\hline std & 0.3390 & 0.3202 & 0.3014 & 0.0318 & 0.0270 & 0.2954 & 0.0448 & 0.0492 & 0.4054 & 0.0277 & 0.0363 & 0.0841 & 0.0516 & 0.0428 & 0.1716 & 0.0383 & 0.0604 & 0.0829 \\
\hline
\end{tabular}


Table 5.4 Correlation of frequency domain HRV parameters (cont.)

LF/HF -- RMSSD

\begin{tabular}{|c|c|c|c|c|c|c|c|c|c|c|c|c|c|c|c|c|c|}
\hline \multirow[t]{2}{*}{ Stat } & \multicolumn{3}{|c|}{ individual } & \multicolumn{3}{|c|}{ Bootstrap-All Infants } & \multicolumn{3}{|c|}{ Bootstrap-ALTE } & \multicolumn{3}{|c|}{ Bootstrap-Preterm } & \multicolumn{3}{|c|}{ Bootstrap-Siblings } & \multicolumn{2}{|c|}{ Boots trap-Healthy } \\
\hline & QS & REM & AWK & QS & REM & AWK & QS & REM & AWK & QS & REM & AWK & QS & REM & AWK & QS & REM AWK \\
\hline & -0.7916 & & & -0.3770 & -0.4130 & & 0.4006 & & & & & & & & & -0.37 & \\
\hline $25.0 \%$ & -0.3396 & -0.458 & & -0.3479 & -0.3873 & & -0.3686 & -0.5251 & -0.4432 & -0.3359 & & & -0.2816 & & & -0.3 & \\
\hline $50.0 \%$ & -0.1027 & -0.3031 & & & -0.3745 & -0.1829 & -0.3550 & -0.5038 & -0.4057 & -0.3166 & -0.3133 & -0.2505 & -0.2591 & -0.3727 & & -0.3307 & \\
\hline $75.0 \%$ & 0.1871 & -0.0476 & -0.0257 & -0.3185 & -0.3607 & -0.0764 & -0.3433 & -0.4825 & 0.5644 & -0.2963 & -0.2961 & -0.1142 & -0.2384 & -0.3566 & 0.2414 & -0.3160 & $-0.4451-0.3484$ \\
\hline $97.5 \%$ & 0.4941 & 0.5435 & & -0.2913 & -0.3323 & 0.3489 & -0.3218 & -0.4365 & 0.6066 & -0.2675 & -0.2616 & -0.0442 & -0.1965 & -0.3238 & 0.3470 & -0.2879 & $-0.3918-0.2586$ \\
\hline std & 0.3457 & 0.3279 & 0.3483 & 0.0217 & 0.0203 & 0.2457 & 0.0196 & 0.0324 & 0.4806 & 0.0282 & 0.0260 & 0.0872 & 0.0331 & 0.0261 & 0.2505 & 0.0214 & 0.04200 .0625 \\
\hline
\end{tabular}

$-0.7920 .702$

LF/HF -- SDNN

\begin{tabular}{|c|c|c|c|c|c|c|c|c|c|c|c|c|c|c|c|c|c|c|}
\hline \multirow[t]{2}{*}{ Stat } & \multicolumn{3}{|c|}{ individual } & \multicolumn{3}{|c|}{ Bootstrap-All Infants } & \multicolumn{3}{|c|}{ Bootstrap-ALTE } & \multicolumn{3}{|c|}{ Bootstrap-Preterm } & \multicolumn{3}{|c|}{ Bootstrap-Siblings } & \multicolumn{3}{|c|}{ Bootstrap-Healthy } \\
\hline & QS & REM & AWK & QS & REM & AWK & QS & REM & AWK & QS & REM & AWK & QS & REM & AWK & QS & REM & AWK \\
\hline $2.5 \%$ & -0.5053 & -0.4453 & -0.3614 & -0.0552 & -0.0572 & 0.0471 & 0.0158 & 0.0105 & -0.0739 & -0.0718 & -0.0459 & 0.0623 & 0.0943 & -0.1733 & 0.0246 & 0.1357 & -0.1736 & -0.1219 \\
\hline $25.0 \%$ & 0.1492 & -0.0944 & -0.0408 & -0.0190 & -0.0255 & 0.1094 & 0.0619 & 0.0699 & -0.0021 & -0.0341 & -0.0073 & 0.1175 & 0.1543 & -0.1107 & 0.1188 & 0.1866 & -0.0926 & -0.0390 \\
\hline $50.0 \%$ & 0.4169 & 0.1454 & 0.1359 & -0.0006 & -0.0069 & 0.3198 & 0.0869 & 0.1101 & 0.0540 & -0.0142 & 0.0140 & 0.1631 & 0.1831 & -0.0796 & 0.2034 & 0.2109 & -0.0520 & 0.0191 \\
\hline $75.0 \%$ & 0.6165 & 0.3899 & 0.3349 & 0.0196 & 0.0119 & 0.4750 & 0.1075 & 0.1469 & 0.9325 & 0.0054 & 0.0392 & 0.4477 & 0.2118 & -0.0525 & 0.6739 & 0.2367 & -0.0099 & 0.0828 \\
\hline $97.5 \%$ & 0.8311 & 0.8084 & 0.9146 & 0.0585 & 0.0489 & 0.8483 & 0.1504 & 0.2269 & 0.9512 & 0.0504 & 0.0862 & 0.5151 & 0.2656 & -0.0071 & 0.7843 & 0.2822 & 0.0688 & 0.2173 \\
\hline std & 0.3572 & 0.3369 & 0.2841 & 0.0293 & 0.0274 & 0.2789 & 0.0339 & 0.0562 & 0.4336 & 0.0304 & 0.0336 & 0.1590 & 0.0433 & 0.0431 & 0.2642 & 0.0377 & 0.0612 & 0.0858 \\
\hline
\end{tabular}

LF/HF -- Median

\begin{tabular}{|c|c|c|c|c|c|c|c|c|c|c|c|c|c|c|c|c|c|}
\hline \multirow[t]{2}{*}{ Stat } & \multicolumn{3}{|c|}{ individual } & \multicolumn{3}{|c|}{ Bootstrap-All Infants } & \multicolumn{3}{|c|}{ Bootstrap-ALTE } & \multicolumn{3}{|c|}{ Bootstrap-Preterm } & \multicolumn{3}{|c|}{ Bootstrap-Siblings } & \multicolumn{2}{|c|}{ Bootstrap-Healthy } \\
\hline & QS & REM & AWK & QS & REM & AWK & QS & REM & AWK & QS & REM & AWK & QS & REM & AWK & QS & REM A K K \\
\hline $2.5 \%$ & -0.6852 & -0.7445 & -0.6168 & -0.2766 & -0.2287 & -0.0794 & -0.2531 & -0.3108 & -0.1614 & -0.2432 & -0.2103 & 0.0028 & -0.1801 & -0.1755 & -0.0121 & -0.2751 & $-0.4525-0.3230$ \\
\hline $25.0 \%$ & -0.4169 & -0.3283 & -0.0189 & -0.2444 & -0.1962 & -0.0071 & -0.2255 & -0.2479 & -0.1441 & -0.2065 & -0.1697 & 0.0420 & -0.1092 & -0.1299 & 0.0569 & -0.2372 & $-0.3915-0.2216$ \\
\hline $50.0 \%$ & -0.2059 & -0.1198 & 0.1810 & -0.2273 & -0.1794 & 0.0312 & -0.2091 & -0.2210 & -0.0347 & -0.1877 & -0.1443 & 0.0661 & -0.0727 & -0.1003 & 0.1021 & -0.2159 & $-0.3589-0.1791$ \\
\hline $75.0 \%$ & 0.0187 & 0.1640 & 0.4024 & -0.2095 & -0.1615 & 0.0589 & -0.1923 & -0.1876 & 0.0076 & -0.1705 & -0.1229 & 0.0926 & -0.0363 & -0.0641 & 0.1700 & -0.1933 & $-0.3214-0.1236$ \\
\hline $97.5 \%$ & 0.5946 & 0.4907 & 0.6769 & -0.1786 & -0.1250 & 0.1030 & -0.1625 & -0.1186 & 0.0815 & -0.1400 & -0.0809 & 0.1438 & 0.0240 & 0.0056 & 0.2744 & -0.1563 & $-0.2474-0.0279$ \\
\hline std & 0.3352 & 0.3067 & 0.3275 & 0.0252 & 0.0263 & 0.0560 & 0.0234 & 0.0474 & 0.0779 & 0.0261 & 0.0334 & 0.0368 & 0.0525 & 0.0484 & 0.0771 & 0.0309 & 0.05090 .0736 \\
\hline
\end{tabular}

$\min \max$ 
Table 5.4 Correlation of frequency domain HRV parameters (cont.)

Total Power -- Median

\begin{tabular}{|c|c|c|c|c|c|c|c|c|c|c|c|c|c|c|c|c|c|c|}
\hline \multirow[t]{2}{*}{ Stat } & \multicolumn{3}{|c|}{ individual } & \multicolumn{3}{|c|}{ Bootstrap-All Infants } & \multicolumn{3}{|c|}{ Bootstrap-ALTE } & \multicolumn{3}{|c|}{ Bootstrap-Preterm } & \multicolumn{3}{|c|}{ Bootstrap-Siblings } & \multicolumn{3}{|c|}{ Bootstrap-Healthy } \\
\hline & QS & REM & AWK & QS & REM & AWK & QS & REM & AWK & QS & REM & A W K & QS & REM & $\mathrm{AWK}$ & $\mathrm{QS}$ & REM & $\mathrm{AWK}$ \\
\hline $2.5 \%$ & -0.2999 & -0.3029 & -0.3620 & 0.4695 & 0.4600 & -0.0537 & 0.4879 & 0.2848 & -0.1439 & 0.4299 & 0.4925 & 0.0638 & 0.4074 & 0.4935 & 0.0464 & 0.2751 & 0.4034 & 0.1275 \\
\hline $25.0 \%$ & 0.0604 & 0.0870 & 0.1008 & 0.5035 & 0.4919 & 0.1099 & 0.5283 & 0.3447 & -0.1281 & 0.4761 & 0.5351 & 0.1448 & 0.4764 & 0.5386 & 0.1402 & 0.3198 & 0.4742 & 0.2414 \\
\hline $50.0 \%$ & 0.3395 & 0.3289 & 0.3160 & 0.5203 & 0.5083 & 0.2037 & 0.5483 & 0.3736 & 0.3388 & 0.5032 & 0.5590 & 0.1916 & 0.5090 & 0.5618 & 0.2898 & 0.3437 & 0.5132 & 0.3012 \\
\hline $75.0 \%$ & 0.4835 & 0.4940 & 0.5850 & 0.5381 & 0.5242 & 0.2708 & 0.5673 & 0.4043 & 0.4078 & 0.5306 & 0.5792 & 0.2345 & 0.5360 & 0.5818 & 0.3814 & 0.3664 & 0.5478 & 0.3467 \\
\hline $97.5 \%$ & 0.8414 & 0.9494 & 0.7712 & 0.5716 & 0.5584 & 0.3719 & 0.6007 & 0.4581 & 0.5007 & 0.5766 & 0.6171 & 0.3299 & 0.5883 & 0.6193 & 0.5043 & 0.4141 & 0.6085 & 0.4482 \\
\hline std & 0.3013 & 0.3145 & 0.3089 & 0.0257 & 0.0246 & 0.1323 & 0.0291 & 0.0452 & 0.2500 & 0.0378 & 0.0324 & 0.0682 & 0.0452 & 0.0315 & 0.1376 & 0.0337 & 0.0540 & 0.0803 \\
\hline
\end{tabular}

$\min \max$

HF -- Median

\begin{tabular}{|c|c|c|c|c|c|c|c|c|c|c|c|c|c|c|c|c|c|c|}
\hline \multirow[t]{2}{*}{ Stat } & \multicolumn{3}{|c|}{ individual } & \multicolumn{3}{|c|}{ Bootstrap-All Infants } & \multicolumn{3}{|c|}{ Bootstrap-ALTE } & \multicolumn{3}{|c|}{ Bootstrap-Preterm } & \multicolumn{3}{|c|}{ Bootstrap-Siblings } & \multicolumn{3}{|c|}{ Bootstrap-Healthy } \\
\hline & QS & REM & AWK & QS & REM & AWK & QS & REM & AWK & QS & REM & AWK & QS & REM & AWK & QS & REM & AWK \\
\hline $2.5 \%$ & -0.2011 & -0.2422 & -0.4731 & 0.5969 & 0.5655 & 0.1487 & 0.6171 & 0.3669 & 0.2854 & 0.5357 & 0.5632 & 0.1474 & 0.5988 & 0.5652 & 0.0444 & 0.4911 & 0.5638 & 0.2339 \\
\hline $25.0 \%$ & 0.3433 & 0.1429 & -0.0 & 0.6244 & 0.5868 & 0.2594 & 0.6457 & 0.4202 & 0.3831 & 0.5823 & 0.5979 & 0.2049 & 0.6482 & 0.5975 & 0.1977 & 0.5197 & 0.6250 & 0.3514 \\
\hline $50.0 \%$ & 0.6024 & 0.4042 & 0.2540 & 0.6403 & 0.5975 & 0.2997 & 0.6591 & 0.4442 & 0.4276 & 0.6129 & 0.6150 & 0.2341 & 0.6702 & 0.6150 & 0.2981 & 0.5330 & 0.6515 & 0.4174 \\
\hline $75.0 \%$ & 0.7552 & 0.6365 & 0.5193 & 0.6574 & 0.6081 & 0.3330 & 0.6728 & 0.4667 & 0.4651 & 0.6392 & 0.6309 & 0.2636 & 0.6885 & 0.6317 & 0.3738 & 0.5455 & 0.6754 & 0.4816 \\
\hline $97.5 \%$ & 0.9163 & 0.8817 & 0.8099 & 0.6831 & 0.6283 & 0.3904 & 0.6946 & 0.5059 & 0.5377 & 0.6878 & 0.6598 & 0.3168 & 0.7233 & 0.6661 & 0.4762 & 0.5727 & 0.7209 & 0.5768 \\
\hline std & 0.3273 & 0.3067 & 0.3627 & 0.0230 & 0.0158 & 0.0633 & 0.0202 & 0.0353 & 0.0625 & 0.0400 & 0.0244 & 0.0436 & 0.0312 & 0.0258 & 0.1219 & 0.0202 & 0.0392 & 0.0901 \\
\hline
\end{tabular}

$-0.4730 .9163$

$\min \max$

LF -- Median

\begin{tabular}{|c|c|c|c|c|c|c|c|c|c|c|c|c|c|c|c|c|c|c|}
\hline \multirow[t]{2}{*}{ Stat } & \multicolumn{3}{|c|}{ individual } & \multicolumn{3}{|c|}{ Bootstrap-All Infants } & \multicolumn{3}{|c|}{ Bootstrap-ALTE } & \multicolumn{3}{|c|}{ Bootstrap-Preterm } & \multicolumn{3}{|c|}{ Bootstrap-Siblings } & \multicolumn{3}{|c|}{ Bootstrap-Healthy } \\
\hline & QS & REM & AWK & QS & REM & AWK & QS & REM & AWK & QS & REM & AWK & QS & REM & AWK & QS & REM & AWK \\
\hline $2.5 \%$ & -0.3857 & -0.3686 & -0.3624 & 0.3539 & 0.4285 & -0.0575 & 0.2972 & 0.2417 & -0.1467 & 0.3740 & 0.4722 & 0.0560 & 0.2919 & 0.4635 & 0.0420 & 0.1200 & 0.3500 & 0.0964 \\
\hline $25.0 \%$ & -0.0018 & & & 0.3927 & & & 0.345 & & -0.1312 & 0.4234 & & & 0.37 & 0.5130 & 0.1364 & 0.1721 & & 03 \\
\hline $50.0 \%$ & 0.1845 & 0.3055 & 0.3226 & 0.4103 & 0.4779 & 0.1885 & 0.3739 & 0.3331 & 0.3038 & 0.4514 & 0.5398 & 0.1794 & 0.4145 & 0.5376 & 0.2768 & 0.1955 & 0.4645 & 0.2695 \\
\hline $75.0 \%$ & 0.4048 & 0.4903 & 0.5841 & 0.4307 & 0.4955 & 0.2505 & 0.3981 & 0.3626 & 0.3677 & 0.4797 & 0.5611 & 0.2227 & 0.4505 & 0.5606 & 0.3686 & 0.2186 & 0.5001 & 0.3146 \\
\hline $97.5 \%$ & 0.7746 & 0.9460 & 0.8081 & 0.4673 & 0.5295 & 0.3529 & 0.4453 & 0.4210 & 0.4643 & 0.5240 & 0.5996 & 0.3196 & 0.5122 & 0.6016 & 0.4925 & 0.2652 & 0.5646 & 0.4084 \\
\hline std & 0.3059 & 0.3175 & 0.3052 & 0.0284 & 0.0261 & 0.1271 & 0.0379 & 0.0462 & 0.2344 & 0.0393 & 0.0335 & 0.0672 & 0.0564 & 0.0349 & 0.1342 & 0.0355 & 0.0563 & 0.0796 \\
\hline
\end{tabular}


Table 5.5 Correlation of corresponding HRV parameter from wavelet and Fourier transform

HF -- HFW

\begin{tabular}{|c|c|c|c|c|c|c|c|c|c|c|c|c|c|c|c|c|c|c|}
\hline \multirow[t]{2}{*}{ Stat } & \multicolumn{3}{|c|}{ individual } & \multicolumn{3}{|c|}{ Bootstrap-All Infants } & \multicolumn{3}{|c|}{ Bootstrap-ALTE } & \multicolumn{3}{|c|}{ Bootstrap-Preterm } & \multicolumn{3}{|c|}{ Bootstrap-Siblings } & \multicolumn{3}{|c|}{ Bootstrap-Healthy } \\
\hline & QS & REM & AWK & QS & REM & AWK & QS & REM & AWK & QS & REM & AWK & QS & REM & AWK & QS & REM & AWK \\
\hline $2.5 \%$ & 0.3752 & 0.3705 & 0.3304 & 0.9464 & 0.8871 & 0.7416 & 0.9706 & 0.8477 & 0.6588 & 0.9030 & 0.8837 & 0.7027 & 0.9117 & 0.9427 & 0.6544 & 0.9481 & 0.7918 & 0.7883 \\
\hline $25.0 \%$ & 0.7778 & 0.6642 & 0.6857 & 0.9608 & 0.8987 & 0.7991 & 0.9754 & 0.8846 & 0.7621 & 0.9289 & 0.9017 & 0.7598 & 0.9346 & 0.9535 & 0.8424 & 0.9607 & 0.8223 & 0.8543 \\
\hline $50.0 \%$ & 0.9075 & 0.7968 & 0.8097 & 0.9672 & 0.9067 & 0.8264 & 0.9774 & 0.9000 & 0.8832 & 0.9390 & 0.9099 & 0.7903 & 0.9479 & 0.9587 & 0.8858 & 0.9680 & 0.8435 & 0.8813 \\
\hline $75.0 \%$ & 0.9557 & 0.8858 & 0.8950 & 0.9719 & 0.9131 & 0.8551 & 0.9794 & 0.9146 & 0.9124 & 0.9476 & 0.9184 & 0.8173 & 0.9578 & 0.9633 & 0.9213 & 0.9748 & 0.8652 & 0.9027 \\
\hline $97.5 \%$ & 0.9942 & 0.9702 & 0.9834 & 0.9785 & 0.9243 & 0.9052 & 0.9828 & 0.9348 & 0.9412 & 0.9630 & 0.9330 & 0.8569 & 0.9729 & 0.9716 & 0.9822 & 0.9821 & 0.9038 & 0.9364 \\
\hline std & 0.1596 & 0.1687 & 0.1755 & 0.0082 & 0.0099 & 0.0416 & 0.0031 & 0.0230 & 0.0879 & 0.0145 & 0.0125 & 0.0403 & 0.0163 & 0.0073 & 0.0767 & 0.0092 & 0.0288 & 0.0371 \\
\hline
\end{tabular}

$\min \max$

LF -- LFW

\begin{tabular}{|c|c|c|c|c|c|c|c|c|c|c|c|c|c|c|c|c|c|c|}
\hline Stat & \multicolumn{3}{|c|}{ individual } & \multicolumn{3}{|c|}{ Bootstrap-All Infants } & \multicolumn{3}{|c|}{ Bootstrap-ALTE } & \multicolumn{3}{|c|}{ Bootstrap-Preterm } & \multicolumn{3}{|c|}{ Bootstrap-Siblings } & \multicolumn{3}{|c|}{ Bootstrap-Healthy } \\
\hline & QS & REM & AWK & QS & REM & AWK & QS & REM & AWK & QS & REM & AWK & QS & REM & AWK & QS & REM & AWK \\
\hline $2.5 \%$ & 0.1588 & 0.1236 & 0.1346 & 0.7752 & 0.7779 & 0.5471 & 0.6837 & 0.6696 & 0.6461 & 0.7945 & 0.7727 & 0.5210 & 0.7154 & 0.7158 & 0.6254 & 0.7287 & 0.7537 & 0.6771 \\
\hline $25.0 \%$ & 0.6240 & 0.4955 & 0.6465 & 0.8170 & 0.8118 & 0.6623 & 0.7270 & 0.7289 & 0.7183 & 0.8413 & 0.8164 & 0.6219 & 0.7972 & 0.7534 & 0.7413 & 0.7753 & 0.8053 & 0.7396 \\
\hline $50.0 \%$ & 0.7736 & 0.6641 & 0.7699 & 0.8390 & 0.8271 & 0.7343 & 0.7511 & 0.7593 & 0.7697 & 0.8652 & 0.8379 & 0.6780 & 0.8409 & 0.7719 & 0.8093 & 0.7963 & 0.8275 & 0.7726 \\
\hline $75.0 \%$ & 0.8710 & 0.8019 & 0.8585 & 0.8593 & 0.8420 & 0.8120 & 0.7710 & 0.7842 & 0.9942 & 0.8869 & 0.8565 & 0.7385 & 0.8740 & 0.7968 & 0.8540 & 0.8160 & 0.8473 & 0.8058 \\
\hline $97.5 \%$ & 0.9781 & 0.9324 & 0.9854 & 0.8918 & 0.8631 & 0.9679 & 0.8103 & 0.8243 & 0.9970 & 0.9216 & 0.8901 & 0.8138 & 0.9187 & 0.8509 & 0.9050 & 0.8538 & 0.8762 & 0.8627 \\
\hline std & 0.2034 & 0.2284 & 0.2174 & 0.0299 & 0.0221 & 0.1234 & 0.0324 & 0.0395 & 0.1319 & 0.0329 & 0.0303 & 0.0787 & 0.0536 & 0.0348 & 0.0745 & 0.0310 & 0.0315 & 0.0479 \\
\hline
\end{tabular}

$\mathrm{LF} / \mathrm{HF}-\mathrm{LFW} / \mathrm{HFW}$

\begin{tabular}{|c|c|c|c|c|c|c|c|c|c|c|c|c|c|c|c|c|c|c|}
\hline \multirow[t]{2}{*}{ Stat } & \multicolumn{3}{|c|}{ individual } & \multicolumn{3}{|c|}{ Bootstrap-All Infants } & \multicolumn{3}{|c|}{ Bootstrap-ALTE } & \multicolumn{3}{|c|}{ Bootstrap-Preterm } & \multicolumn{3}{|c|}{ Bootstrap-Siblings } & \multicolumn{3}{|c|}{ Bootstrap-Healthy } \\
\hline & QS & REM & AWK & QS & REM & AWK & QS & REM & AWK & QS & REM & AWK & QS & REM & AWK & QS & REM & AWK \\
\hline $2.5 \%$ & 0.0477 & 0.0923 & 0.2421 & 0.6837 & 0.7064 & 0.2765 & 0.5063 & 0.6899 & 0.3744 & 0.7319 & 0.7134 & 0.5006 & 0.7753 & 0.6202 & 0.3766 & 0.8700 & 0.6999 & 0.5343 \\
\hline $25.0 \%$ & 0.5935 & 0.3400 & 0.5038 & 0.7416 & 0.7371 & 0.5671 & 0.5382 & 0.7310 & 0.4416 & 0.7765 & 0.7546 & 0.6015 & 0.8054 & 0.6784 & 0.4955 & 0.8932 & 0.7666 & 0.7459 \\
\hline $50.0 \%$ & 0.7529 & 0.5867 & 0.6527 & 0.7821 & 0.7547 & 0.6488 & 0.5601 & 0.7561 & 0.7268 & 0.7987 & 0.7773 & 0.6544 & 0.8228 & 0.7030 & 0.5552 & 0.9033 & 0.8059 & 0.8222 \\
\hline $75.0 \%$ & 0.8527 & 0.7007 & 0.7817 & 0.8117 & 0.7720 & 0.6961 & 0.5849 & 0.7795 & 0.7824 & 0.8241 & 0.7986 & 0.7020 & 0.8421 & 0.7319 & 0.5989 & 0.9119 & 0.8366 & 0.8737 \\
\hline $97.5 \%$ & 0.9599 & 0.9700 & 0.9255 & 0.8489 & 0.7989 & 0.7679 & 0.7050 & 0.8191 & 0.8501 & 0.8651 & 0.8357 & 0.7766 & 0.8752 & 0.7909 & 0.6804 & 0.9268 & 0.8777 & 0.9233 \\
\hline std & 0.2263 & 0.2388 & 0.1954 & 0.0458 & 0.0247 & 0.1603 & 0.0449 & 0.0345 & 0.1672 & 0.0342 & 0.0319 & 0.0706 & 0.0263 & 0.0448 & 0.0790 & 0.0144 & 0.0483 & 0.1040 \\
\hline
\end{tabular}

$\min \max$ 
Table 5.6 Correlation between nonlinear HRV parameters and others

ApEn -- Poincare

\begin{tabular}{|c|c|c|c|c|c|c|c|c|c|c|c|c|c|c|c|c|c|}
\hline \multirow[t]{2}{*}{ Stat } & \multicolumn{3}{|c|}{ individual } & \multicolumn{3}{|c|}{ Bootstrap-All Infants } & \multicolumn{3}{|c|}{ Bootstrap-ALTE } & \multicolumn{3}{|c|}{ Bootstrap-Preterm } & \multicolumn{3}{|c|}{ Bootstrap-Siblings } & \multicolumn{2}{|c|}{ Bootstrap-Healthy } \\
\hline & QS & REM & AWK & QS & REM & AWK & QS & REM & AWK & QS & REM & AWK & QS & REM & AWK & QS & REM AW K \\
\hline $2.5 \%$ & -0.9123 & -0.9055 & -0.7908 & -0.3261 & -0.2726 & -0.4033 & -0.2425 & -0.4071 & -0.4902 & -0.3056 & -0.2767 & -0.3546 & -0.4782 & -0.3487 & -0.5486 & -0.2808 & $-0.2048-0.5026$ \\
\hline $25.0 \%$ & -0.7599 & -0.6923 & -0.6521 & -0.2946 & -0.2445 & -0.3742 & -0.1996 & -0.3519 & -0.4489 & -0.2659 & -0.2448 & -0.3173 & -0.4395 & -0.3007 & -0.4721 & -0.2287 & $-0.1346-0.443$ \\
\hline $50.0 \%$ & -0.5741 & -0.4375 & -0.4837 & -0.2769 & -0.2305 & -0.3572 & -0.1752 & -0.3241 & -0.4236 & -0.2452 & -0.2294 & -0.3000 & -0.4188 & -0.2765 & -0.4355 & -0.1964 & $-0.1002-0.413$ \\
\hline $75.0 \%$ & -0.3536 & -0.1479 & -0.3110 & -0.2599 & -0.2160 & -0.3411 & -0.1512 & -0.2927 & -0.3983 & -0.2228 & -0.2132 & -0.2810 & -0.3965 & -0.2485 & -0.3950 & -0.1617 & -0.05 \\
\hline $97.5 \%$ & 0.6184 & 0.6053 & 0.0474 & -0.2265 & -0.1886 & -0.3099 & -0.1060 & -0.2313 & -0.3396 & -0.1787 & -0.1797 & -0.2439 & -0.3523 & -0.1816 & -0.2985 & -0.0982 & $0.0008-0.3113$ \\
\hline std & 0.3639 & 0.3963 & 0.2322 & 0.0260 & 0.0212 & 0.0248 & 0.0354 & 0.0437 & 0.0379 & 0.0321 & 0.0245 & 0.0277 & 0.0318 & 0.0418 & 0.0600 & 0.0480 & 0.05470 .047 \\
\hline
\end{tabular}

ApEn -- FD

\begin{tabular}{|c|c|c|c|c|c|c|c|c|c|c|c|c|c|c|c|c|c|c|}
\hline \multirow[t]{2}{*}{ Stat } & \multicolumn{3}{|c|}{ individual } & \multicolumn{3}{|c|}{ Bootstrap-All Infants } & \multicolumn{3}{|c|}{ Bootstrap-ALTE } & \multicolumn{3}{|c|}{ Bootstrap-Preterm } & \multicolumn{3}{|c|}{ Bootstrap-Siblings } & \multicolumn{3}{|c|}{ Bootstrap-Healthy } \\
\hline & QS & REM & AWK & QS & REM & AWK & QS & REM & AWK & QS & REM & AWK & QS & REM & AWK & QS & REM & AWK \\
\hline $2.5 \%$ & -0.7000 & -0.7159 & -0.3949 & 0.1270 & 0.4155 & 0.4190 & 0.3037 & 0.4414 & 0.3681 & 0.1445 & 0.4050 & 0.4263 & 0.0077 & 0.2971 & 0.1810 & 0.2477 & 0.3917 & 0.3965 \\
\hline $25.0 \%$ & -0.3873 & -0.1623 & -0.0091 & 0.1585 & 0.4478 & 0.4572 & 0.3381 & 0.5220 & 0.4342 & 0.1866 & 0.4418 & 0.4689 & 0.0592 & 0.3474 & 0.3048 & 0.2878 & 0.4423 & 0.4777 \\
\hline $50.0 \%$ & -0.0045 & 0.1790 & 0.2652 & 0.1782 & 0.4625 & 0.4792 & 0.3559 & 0.5604 & 0.4756 & 0.2093 & 0.4606 & 0.4886 & 0.0867 & 0.3728 & 0.3682 & 0.3105 & 0.4682 & 0.5189 \\
\hline $75.0 \%$ & 0.2637 & 0.4971 & 0.4931 & 0.1965 & 0.4778 & 0.4996 & 0.3738 & 0.6029 & 0.5160 & 0.2343 & 0.4812 & 0.5056 & 0.1185 & 0.3993 & 0.4264 & 0.3320 & 0.4936 & 0.5530 \\
\hline $97.5 \%$ & 0.7390 & 0.9106 & 0.8024 & 0.2312 & 0.5093 & 0.5388 & 0.4040 & 0.6671 & 0.5884 & 0.2792 & 0.5135 & 0.5415 & 0.1722 & 0.4483 & 0.5313 & 0.3709 & 0.5394 & 0.6283 \\
\hline std & 0.4071 & 0.4491 & 0.3453 & 0.0269 & 0.0234 & 0.0310 & 0.0260 & 0.0585 & 0.0575 & 0.0345 & 0.0283 & 0.0284 & 0.0422 & 0.0395 & 0.0878 & 0.0318 & 0.0373 & 0.0595 \\
\hline
\end{tabular}

FD -- Poincare

\begin{tabular}{|c|c|c|c|c|c|c|c|c|c|c|c|c|c|c|c|c|c|c|}
\hline \multirow[t]{2}{*}{ Stat } & \multicolumn{3}{|c|}{ individual } & \multicolumn{3}{|c|}{ Bootstrap-All Infants } & \multicolumn{3}{|c|}{ Bootstrap-ALTE } & \multicolumn{3}{|c|}{ Bootstrap-Preterm } & \multicolumn{3}{|c|}{ Bootstrap-Siblings } & \multicolumn{3}{|c|}{ Bootstrap-Healthy } \\
\hline & QS & REM & $\mathrm{AWK}$ & QS & REM & AWK & QS & REM & AWK & QS & REM & AWK & QS & REM & AWK & QS & REM & AWK \\
\hline $2.5 \%$ & 0.0613 & -0.4077 & -0.1134 & 0.7009 & 0.5292 & 0.2513 & 0.7102 & 0.2791 & 0.1770 & 0.6792 & 0.5060 & 0.3007 & 0.6479 & 0.5983 & 0.1910 & 0.6111 & 0.6244 & 0.1379 \\
\hline $25.0 \%$ & 0.5619 & 0.3975 & 0.2167 & 0.7333 & 0.5542 & 0.2953 & 0.7373 & 0.3336 & 0.2678 & 0.7039 & 0.5360 & 0.3365 & 0.6924 & 0.6374 & 0.2910 & 0.6598 & 0.6742 & 0.2456 \\
\hline $50.0 \%$ & 0.6993 & 0.5921 & 0.3678 & 0.7511 & 0.5681 & 0.3167 & 0.7540 & 0.3627 & 0.3240 & 0.7186 & 0.5507 & 0.3562 & 0.7139 & 0.6605 & 0.3468 & 0.6907 & 0.7000 & 0.2989 \\
\hline $75.0 \%$ & 0.8293 & 0.7422 & 0.5268 & 0.7640 & 0.5816 & 0.3391 & 0.7693 & 0.3983 & 0.3737 & 0.7349 & 0.5651 & 0.3761 & 0.7358 & 0.6875 & 0.3964 & 0.7199 & 0.7254 & 0.3567 \\
\hline $97.5 \%$ & 0.9341 & 0.9114 & 0.7793 & 0.7884 & 0.6040 & 0.3818 & 0.7952 & 0.4657 & 0.4606 & 0.7649 & 0.5941 & 0.4131 & 0.7642 & 0.7373 & 0.5071 & 0.7732 & 0.7676 & 0.4402 \\
\hline std & 0.2212 & 0.3137 & 0.2542 & 0.0227 & 0.0195 & 0.0336 & 0.0221 & 0.0463 & 0.0742 & 0.0225 & 0.0225 & 0.0297 & 0.0306 & 0.0364 & 0.0807 & 0.0424 & 0.0372 & 0.0784 \\
\hline
\end{tabular}

$\min \max$ 
Table 5.6 Correlation between nonlinear HRV parameters and others (cont.)

ApEn -- HF

\begin{tabular}{|c|c|c|c|c|c|c|c|c|c|c|c|c|c|c|c|c|c|c|}
\hline Stat & \multicolumn{3}{|c|}{ individual } & \multicolumn{3}{|c|}{ Bootstrap-All Infants } & \multicolumn{3}{|c|}{ Bootstrap-ALTE } & \multicolumn{3}{|c|}{ Bootstrap-Preterm } & \multicolumn{3}{|c|}{ Bootstrap-Siblings } & \multicolumn{3}{|c|}{ Bootstrap-Healthy } \\
\hline & QS & REM & AWK & QS & REM & AWK & QS & REM & AWK & $\overline{Q S}$ & REM & AWK & QS & REM & AWK & QS & REM & AWK \\
\hline $2.5 \%$ & 0.7513 & -0.6280 & -0.5441 & -0.0723 & 0.0792 & -0.0296 & 0.0373 & 0.1907 & 0.1501 & -0.0885 & 0.0413 & -0.0031 & -0.1799 & -0.0334 & -0.2646 & 0.1093 & 0.1057 & 0.0536 \\
\hline $25.0 \%$ & 0.4271 & -0.2505 & -0.2118 & -0.0438 & 0.1050 & 0.0845 & 0.0675 & 0.2454 & 0.2057 & -0.0464 & 0.0797 & 0.0572 & -0.1408 & 0.0105 & -0.0350 & 0.1440 & 0.1719 & 0.1425 \\
\hline $50.0 \%$ & -0.218 & -0.0059 & 0.0328 & -0.0299 & 0.1197 & 0.1258 & 0.0821 & 0.2729 & 0.2432 & -0.0228 & 0.1002 & 0.0925 & -0.1162 & 0.0337 & 0.0847 & 0.1638 & 0.2051 & 0.1899 \\
\hline $75.0 \%$ & 0.0411 & 0.2720 & 0.2418 & -0.0151 & 0.1351 & 0.1600 & 0.0964 & 0.3009 & 0.2764 & -0.0013 & 0.1200 & 0.1320 & -0.0892 & 0.0537 & 0.1731 & 0.1832 & 0.2421 & 0.24 \\
\hline $97.5 \%$ & 0.7209 & 0.7233 & 0.6771 & 0.0130 & 0.1609 & 0.2175 & 0.1262 & 0.3565 & 0.3388 & 0.0460 & 0.1631 & 0.1865 & -0.0339 & 0.1043 & 0.2752 & 0.2268 & 0.3083 & 0.3488 \\
\hline std & 0.3831 & 0.3645 & 0.3099 & 0.0217 & 0.0214 & 0.0641 & 0.0221 & 0.0423 & 0.0495 & 0.0349 & 0.0303 & 0.0512 & 0.0376 & 0.0336 & 0.1482 & 0.0296 & 0.0516 & 0.0772 \\
\hline
\end{tabular}

\begin{tabular}{|c|c|c|c|c|c|c|c|c|c|c|c|c|c|c|c|c|c|c|}
\hline \multicolumn{19}{|c|}{ ApEn -- LF } \\
\hline Stat & \multicolumn{3}{|c|}{ individual } & \multicolumn{3}{|c|}{ Bootstrap-All Infants } & \multicolumn{3}{|c|}{ strap-ALTE } & \multicolumn{3}{|c|}{ Bootstrap-Preterm } & \multicolumn{3}{|c|}{ Bootstrap-Siblings } & \multicolumn{3}{|c|}{ Bootstrap-Healthy } \\
\hline & QS & REM & AWK & QS & REM & AWK & QS & REM & AWK & QS & REM & AWK & QS & REM & AWK & QS & REM & AWK \\
\hline $2.5 \%$ & -0.8433 & $3-0.7608$ & -0.6815 & -0.3777 & -0.2397 & -0.2518 & -0.3888 & -0.3204 & -0.2563 & -0.3520 & -0.2599 & -0.2574 & -0.4977 & -0.2497 & -0.3904 & -0.4220 & -0.2707 & -0.3758 \\
\hline $25.0 \%$ & -0.648 & -0.4787 & -0.3996 & -0.3500 & -0.2145 & -0.2203 & -0.352 & -0.2616 & -0.2172 & -0.3132 & -0.2282 & -0.2287 & -0.4419 & -0.2018 & -0.3015 & -0.3789 & -0.2087 & -0.3117 \\
\hline $50.0 \%$ & -0.507 & -0 & 308 & -0.33 & -0.2 & -0.1 & -0.3 & & -0.2022 & -0.294 & . & -0.2085 & -0.4130 & -0.1 & -0.2443 & -0.3537 & & -0 . \\
\hline $75.0 \%$ & -0.3321 & -0.1077 & -0.0381 & -0.3163 & -0.1857 & -0.1645 & -0.3127 & -0.2017 & -0.1516 & -0.2749 & 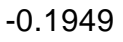 & -0.1867 & -0.3884 & -0.1482 & -0.1548 & -0.3286 & -0.1511 & -0.2355 \\
\hline $97.5 \%$ & 0.2385 & 0.2519 & 0.3894 & -0.2823 & $3-0.1600$ & -0.1302 & -0.2731 & -0.1452 & -0.0900 & -0.2388 & -0.1628 & -0.1432 & -0.3503 & -0.0983 & -0.0240 & -0.2769 & -0.0893 & -0.1681 \\
\hline std & 0.2829 & 0.2767 & 0.2764 & 0.0245 & 0.0212 & 0.0359 & 0.0295 & 0.0449 & 0.0454 & 0.0293 & 0.0250 & 0.0296 & 0.0386 & 0.0391 & 0.0993 & 0.0373 & 0.0442 & 0.0547 \\
\hline
\end{tabular}

ApEn - Total Power

\begin{tabular}{|c|c|c|c|c|c|c|c|c|c|c|c|c|c|c|c|c|}
\hline Stat & \multicolumn{2}{|c|}{ individual } & \multicolumn{3}{|c|}{ Bootstrap-All Infants } & \multicolumn{2}{|c|}{ Bootstrap-ALTE } & \multicolumn{3}{|c|}{ Bootstrap-Preterm } & \multicolumn{3}{|c|}{ Bootstrap-Siblings } & \multicolumn{3}{|c|}{ Bootstrap-Healthy } \\
\hline & REM & AWK & QS & REM & AWK & QS $\quad$ REM & AWK & QS & REM & $\mathrm{AWK}$ & QS & REM & AWK & QS & REM & $\mathrm{AWK}$ \\
\hline $2.5 \%$ & $-0.8367-0.7216$ & -0.6589 & -0.3279 & -0.2098 & -0.2310 & $-0.2755-0.2515$ & -0.2279 & -0.3109 & -0.2390 & -0.2415 & -0.4396 & -0.2223 & -0.3738 & -0.3199 & -0.2149 & -0.3428 \\
\hline $25.0 \%$ & $-0.6351-0.4606$ & -0.4101 & -0.3001 & -0.1821 & -0.1967 & $-0.2394-0.1983$ & -0.2112 & -0.2763 & -0.2045 & -0.2072 & -0.3927 & -0.1743 & -0.2855 & -0.2722 & -0.1604 & -0.2706 \\
\hline $50.0 \%$ & $-0.4928-0.3180$ & -0.2626 & -0.2845 & -0.1673 & -0.1739 & $-0.2197-0.166$ & -0.1487 & -0.2574 & -0.18 & 372 & -0.3713 & -0.1495 & -0.2129 & -0.2452 & -0.1306 & -0.2348 \\
\hline $75.0 \%$ & $-0.2657-0.0861$ & -0.0113 & -0.2691 & -0.1537 & -0.1459 & $-0.2004-0.1384$ & -0.1031 & -0.2339 & -0.1704 & -0.1654 & -0.3521 & -0.1259 & -0.1165 & -0.2180 & -0.1022 & -0.1931 \\
\hline $97.5 \%$ & 0.44670 .3119 & 0.3690 & -0.2377 & -0.1246 & -0.1224 & $-0.1616-0.0807$ & -0.0339 & -0.1945 & -0.1374 & -0.1152 & -0.3161 & -0.0775 & 0.0121 & -0.1694 & -0.0454 & -0.1178 \\
\hline std & $0.3170 \quad 0.2836$ & 0.2828 & 0.0229 & 0.0218 & 0.0318 & 0.02910 .0441 & 0.0607 & 0.0305 & 0.0257 & 0.0316 & 0.0313 & 0.0368 & 0.1073 & 0.0382 & 0.0447 & 0.0571 \\
\hline
\end{tabular}

ApEn - LF/HF

\begin{tabular}{|c|c|c|c|c|c|c|c|c|c|c|c|c|c|c|c|c|c|c|}
\hline Stat & \multicolumn{3}{|c|}{ individual } & \multicolumn{3}{|c|}{ Bootstrap-All Infants } & \multicolumn{3}{|c|}{ Bootstrap-ALTE } & \multicolumn{3}{|c|}{ Bootstrap-Preterm } & \multicolumn{3}{|c|}{ Bootstrap-Siblings } & \multicolumn{3}{|c|}{ Bootstrap-Healthy } \\
\hline & QS & REM & AWK & QS & REM & AWK & QS & REM & AWK & QS & REM & AWK & QS & REM & AWK & QS & REM & AWK \\
\hline $2.5 \%$ & -0.8980 & -0.8344 & -0.7837 & -0.5295 & -0.5647 & -0.4347 & -0.5802 & -0.6389 & -0.4826 & -0.5509 & -0.5520 & -0.4350 & -0.6214 & -0.6413 & -0.4881 & -0.6173 & -0.6573 & -0.5371 \\
\hline $25.0 \%$ & -0.7025 & -0.6204 & -0.4865 & -0.4933 & -0.5403 & -0.4015 & -0.5485 & -0.6018 & -0.4223 & -0.5027 & -0.5152 & -0.4047 & -0.5828 & -0.6039 & -0.4330 & -0.5882 & -0.6151 & -0.4649 \\
\hline $50.0 \%$ & -0.5945 & -0.4 & 267 & -0.4722 & 40 & -0.3828 & -0.5265 & 802 & -0.3644 & -0.4707 & -0.4 & -0.3 & -0.5649 & -0.5798 & -0.4002 & -0.5735 & -0.59 & -0.4275 \\
\hline $75.0 \%$ & -0.3763 & -0.2212 & -0.2099 & -0.4525 & -0.5031 & -0.3533 & -0.5082 & -0.5565 & -0.2395 & -0.4459 & -0.4657 & -0.3739 & -0.5476 & -0.5486 & -0.3569 & -0.5591 & -0.5682 & -0.3941 \\
\hline $97.5 \%$ & 0.4494 & 0.2339 & 0.2312 & -0.4142 & -0.4609 & -0.1480 & -0.4795 & -0.5072 & -0.2238 & -0.3987 & -0.4133 & -0.3436 & -0.5069 & -0.4894 & -0.2725 & -0.5296 & -0.5197 & -0.3364 \\
\hline std & 0.3189 & 0.2836 & 0.2389 & 0.0297 & 0.0273 & 0.0992 & 0.0276 & 0.0334 & 0.0886 & 0.0395 & 0.0363 & 0.0235 & 0.0280 & 0.0393 & 0.0554 & 0.0224 & 0.0351 & 0.0514 \\
\hline
\end{tabular}


Table 5.6 Correlation between nonlinear HRV parameters and others (cont.)

ApEn -- RMSSD

\begin{tabular}{|c|c|c|c|c|c|c|c|c|c|c|c|c|c|c|c|c|c|c|}
\hline Stat & \multicolumn{3}{|c|}{ individual } & \multicolumn{3}{|c|}{ Bootstrap-All Infants } & \multicolumn{3}{|c|}{ Bootstrap-ALTE } & \multicolumn{3}{|c|}{ Bootstrap-Preterm } & \multicolumn{3}{|c|}{ Bootstrap-Siblings } & \multicolumn{3}{|c|}{ Bootstrap-Healthy } \\
\hline & QS & REM & AWK & QS & REM & AWK & QS & REM & AWK & QS & REM & AWK & QS & REM & AWK & QS & REM & AWK \\
\hline $2.5 \%$ & -0.7945 & -0.6882 & -0.4950 & -0.0194 & 0.2077 & 0.1965 & 0.1473 & 0.3007 & 0.1126 & -0.0186 & 0.1730 & 0.1972 & -0.1576 & 0.0785 & -0.0989 & 0.1878 & 0.2472 & 0.2569 \\
\hline $25.0 \%$ & -0.5040 & -0.3073 & -0.1751 & 0.0116 & 0.2381 & 0.2536 & 0.1791 & 0.3730 & 0.2025 & 0.0294 & 0.2117 & 0.2473 & -0.1111 & 0.1301 & 0.0584 & 0.2278 & 0.3061 & 3313 \\
\hline $50.0 \%$ & -0.1541 & 0.0213 & 0.1066 & 0.0283 & 0.2545 & 0.2858 & 0.1964 & 0.4076 & 0.3415 & 0.0557 & 0.2318 & 0.2718 & -0.0853 & 0.1557 & 0.1580 & 0.2469 & 0.3344 & 0.3709 \\
\hline $75.0 \%$ & 0.1205 & 0.4152 & 0.3407 & 0.0470 & 0.2701 & 0.3143 & 0.2125 & 0.4458 & 0.3982 & 0.0815 & 0.2505 & 0.2951 & -0.0588 & 0.1790 & 0.2566 & 0.2685 & 0.3608 & 0.4104 \\
\hline $97.5 \%$ & 0.7296 & 0.8734 & 0.7697 & 0.0820 & 0.2953 & 0.3594 & 0.2442 & 0.5117 & 0.4831 & 0.1310 & 0.2841 & 0.3399 & -0.0042 & 0.2258 & 0.3875 & 0.3114 & 0.4160 & 0.4896 \\
\hline std & 0.4103 & 0.4367 & 0.3612 & 0.0258 & 0.0228 & 0.0430 & 0.0249 & 0.0537 & 0.1112 & 0.0381 & 0.0285 & 0.0361 & 0.0402 & 0.0370 & 0.1298 & 0.0316 & 0.0418 & 0.0579 \\
\hline
\end{tabular}

ApEn -- SDNN

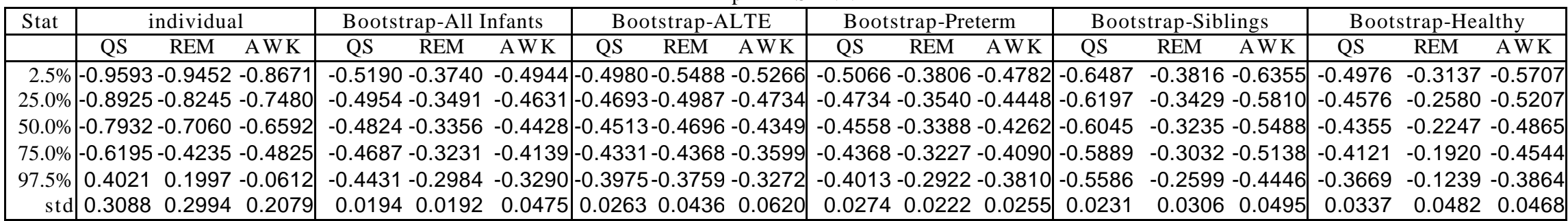

ApEn -- IQRNN

\begin{tabular}{|c|c|c|c|c|c|c|c|c|c|c|c|c|c|c|c|c|c|c|}
\hline Stat & \multicolumn{3}{|c|}{ individual } & \multicolumn{3}{|c|}{ Bootstrap-All Infants } & \multicolumn{3}{|c|}{ Bootstrap-ALTE } & \multicolumn{3}{|c|}{ Bootstrap-Preterm } & \multicolumn{3}{|c|}{ Bootstrap-Siblings } & \multicolumn{3}{|c|}{ Bootstrap-Healthy } \\
\hline & QS & REM & AWK & $\mathrm{QS}$ & REM & AWK & QS & REM & AWK & QS & REM & AWK & QS & REM & AWK & QS & REM & AWK \\
\hline $2.5 \%$ & -0.9081 & -0.9182 & -0.7993 & -0.3874 & -0.3122 & -0.3793 & -0.3320 & -0.4778 & -0.4696 & -0.3456 & -0.3075 & -0.3309 & -0.5364 & -0.3683 & -0.4963 & -0.3852 & -0.2829 & -0.4946 \\
\hline $25.0 \%$ & -0.7453 & -0.7019 & -0.6164 & -0.3536 & -0.2888 & -0.3482 & -0.2937 & -0.4348 & -0.4153 & -0.3068 & -0.2776 & -0.2956 & -0.4953 & -0.3213 & -0.4220 & -0.3345 & -0.2113 & -0.4347 \\
\hline $50.0 \%$ & -0.5997 & -0.4912 & -0.4414 & -0.3373 & -0.2746 & -0.3331 & -0.2690 & -0.404 & -0.3896 & -0.2845 & -0.26 & -0.2744 & -0.4753 & -0.2934 & -0.3785 & -0.3023 & -0.1771 & -0.4009 \\
\hline $75.0 \%$ & -0.4225 & -0.1677 & -0.2472 & -0.3197 & -0.2611 & -0.3151 & -0.2450 & -0.3751 & -0.3635 & -0.2645 & -0.2430 & -0.2576 & -0.4560 & -0.2637 & -0.3342 & -0.2714 & -0.1428 & -0.3667 \\
\hline $97.5 \%$ & 0.5659 & 0.4534 & 0.1265 & -0.2885 & -0.2322 & -0.2838 & -0.2010 & -0.3107 & -0.3031 & -0.2196 & -0.2093 & -0.2210 & -0.4201 & -0.1923 & -0.2434 & -0.2081 & -0.0684 & -0.2986 \\
\hline std & 0.3516 & 0.3670 & 0.2449 & 0.0255 & 0.0206 & 0.0244 & 0.0343 & 0.0423 & 0.0410 & 0.0317 & 0.0250 & 0.0276 & 0.0295 & 0.0436 & 0.0649 & 0.0446 & 0.0528 & 0.0493 \\
\hline
\end{tabular}

ApEn -- Median

\begin{tabular}{|c|c|c|c|c|c|c|c|c|c|c|c|c|c|c|c|c|c|c|}
\hline Stat & \multicolumn{3}{|c|}{ individual } & \multicolumn{3}{|c|}{ Bootstrap-All Infants } & \multicolumn{3}{|c|}{ Bootstrap-ALTE } & \multicolumn{3}{|c|}{ Bootstrap-Preterm } & \multicolumn{3}{|c|}{ Bootstrap-Siblings } & \multicolumn{3}{|c|}{ Bootstrap-Healthy } \\
\hline & QS & REM & AWK & QS & REM & AWK & QS & REM & AWK & $\mathrm{QS}$ & REM & AWK & QS & REM & AWK & QS & REM & AWK \\
\hline $2.5 \%$ & -0.7095 & -0.7361 & -0.6311 & -0.1969 & -0.0336 & 0.0750 & -0.1908 & -0.1141 & 0.0103 & -0.1537 & -0.0580 & 0.0654 & -0.2852 & -0.1855 & 0.0114 & 0.0663 & 0.0189 & 0.0732 \\
\hline $25.0 \%$ & -0.2934 & -0.1 & -0.0703 & $-0.163 \varsigma$ & -0.0027 & 0.1119 & -0.149 & 0431 & 0.0810 & -0.1117 & -0.0185 & 0.1045 & -0.2351 & -0.1027 & 0.1366 & 0.1091 & 0.0969 & 0.1804 \\
\hline $50.0 \%$ & -0.0210 & 0.1405 & 0.1392 & -0.1488 & 0.0148 & 0.1327 & -0.1305 & -0.0098 & 0.1177 & -0.0902 & 0.0022 & 0.1268 & -0.2070 & -0.0662 & 0.1914 & 0.1349 & 0.1402 & 0.2361 \\
\hline $75.0 \%$ & 0.2172 & 0.4135 & 0.4548 & -0.1332 & 0.0323 & 0.1556 & -0.1075 & 0.0248 & 0.1586 & -0.0709 & 0.0222 & 0.1464 & -0.1814 & -0.0320 & 0.2504 & 0.1616 & 0.1852 & 0.2921 \\
\hline $97.5 \%$ & 0.6902 & 0.7716 & 0.8432 & -0.1001 & 0.0640 & 0.1985 & -0.0672 & 0.0946 & 0.2358 & -0.0289 & 0.0606 & 0.1843 & -0.1223 & 0.0241 & 0.3545 & 0.2148 & 0.2671 & 0.3958 \\
\hline std & 0.3797 & 0.3940 & 0.3777 & 0.0244 & 0.0252 & 0.0321 & 0.0312 & 0.0517 & 0.0568 & 0.0315 & 0.0298 & 0.0312 & 0.0415 & 0.0543 & 0.0853 & 0.0375 & 0.0652 & 0.0815 \\
\hline
\end{tabular}


Table 5.6 Correlation between nonlinear HRV parameters and others (cont.)

Poincare -- HF

\begin{tabular}{|c|c|c|c|c|c|c|c|c|c|c|c|c|c|c|c|c|c|c|}
\hline Stat & \multicolumn{3}{|c|}{ individual } & \multicolumn{3}{|c|}{ Bootstrap-All Infants } & \multicolumn{3}{|c|}{ Bootstrap-ALTE } & \multicolumn{3}{|c|}{ Bootstrap-Preterm } & \multicolumn{3}{|c|}{ Bootstrap-Siblings } & \multicolumn{3}{|c|}{ Bootstrap-Healthy } \\
\hline & $\mathrm{QS}$ & REM & AWK & QS & REM & $\mathrm{AWK}$ & QS & REM & $\mathrm{AWK}$ & $\mathrm{QS}$ & REM & AWK & $\mathrm{QS}$ & REM & AWK & $\mathrm{QS}$ & REM & $\mathrm{AWK}$ \\
\hline $2.5 \%$ & 0.0745 & -0.2229 & -0.1376 & 0.6888 & 0.5665 & 0.1793 & 0.7422 & 0.2681 & 0.1068 & 0.7320 & 0.5874 & 0.2690 & 0.6073 & 0.6136 & 0.1213 & 0.5620 & 0.5551 & 0.1762 \\
\hline $25.0 \%$ & 0.5459 & 0.2389 & 0.1544 & 0.7306 & 0.5993 & 0.2849 & 0.7696 & 0.3174 & 0.1777 & 0.7728 & 0.6263 & 0.3318 & 0.7020 & 0.6702 & 0.2696 & 0.6151 & 0.6233 & 0.2862 \\
\hline $50.0 \%$ & 0.7017 & 0.4517 & 0.3582 & 0.7496 & 0.6157 & 0.3242 & 0.7867 & 0.3521 & 0.2373 & 0.7948 & 0.6472 & 0.3591 & 0.7475 & 0.7040 & 0.3525 & 0.6491 & 0.6616 & 0.3485 \\
\hline $75.0 \%$ & 0.8296 & 0.6562 & 0.5374 & 0.7652 & 0.6349 & 0.3535 & 0.8024 & 0.3889 & 0.2937 & 0.8170 & 0.6697 & 0.3894 & 0.7788 & 0.7324 & 0.4297 & 0.6804 & & 0. \\
\hline $97.5 \%$ & 0.9500 & 0.8953 & 0.8806 & 0.7916 & 0.6664 & 0.4124 & 0.8253 & 0.4543 & 0.3829 & 0.8561 & 0.7139 & 0.4432 & 0.8309 & 0.7856 & 0.5754 & 0.7278 & 0.7632 & 0.5238 \\
\hline std & 0.2186 & 0.2812 & 0.2630 & 0.0269 & 0.0260 & 0.0585 & 0.0222 & 0.0491 & 0.0755 & 0.0315 & 0.0319 & 0.0431 & 0.0570 & 0.0442 & 0.1196 & 0.0447 & 0.0549 & 0.0914 \\
\hline
\end{tabular}

Poincare -- LF

\begin{tabular}{|c|c|c|c|c|c|c|c|c|c|c|c|c|c|c|c|c|c|c|}
\hline Stat & \multicolumn{3}{|c|}{ individual } & \multicolumn{3}{|c|}{ Bootstrap-All Infants } & \multicolumn{3}{|c|}{ Bootstrap-ALTE } & \multicolumn{3}{|c|}{ Bootstrap-Preterm } & \multicolumn{3}{|c|}{ Bootstrap-Siblings } & \multicolumn{3}{|c|}{ Bootstrap-Healthy } \\
\hline & QS & REM & AWK & QS & REM & AWK & QS & REM & AWK & QS & REM & AWK & QS & REM & AWK & QS & REM & AWK \\
\hline $2.5 \%$ & -0.007 & -0.2714 & -0.1003 & 0.6299 & 0.5925 & 0.1982 & 0.5666 & 0.3799 & 0.2796 & 0.6648 & 0.5576 & 0.173 & 0.5893 & 0.6043 & 0.1573 & 0.5398 & 0.6155 & 0.2991 \\
\hline 25.0 & $44 / 8$ & 0.2619 & 0.1 & 0 & 0.6321 & 0 & 6063 & & & 0.7222 & 0.6111 & & & & & & & \\
\hline $50.0 \%$ & 0.6374 & 0.4693 & 0.3340 & 0.6971 & 0.6570 & 0.3627 & 0.6289 & 0.4810 & 0.4945 & 0.7601 & 0.6412 & 0.2902 & 0.6898 & 0.6896 & 0.4763 & 0.6132 & 0.7182 & 0.4518 \\
\hline $75.0 \%$ & 0.7853 & 0.6639 & 0.5724 & 0.7198 & 0.6769 & 0.4333 & .6534 & 0.5162 & 0.5970 & 0.7910 & 0.6699 & 0.3467 & 0.7276 & 0.7140 & 0.55 & 0.6398 & 0.7485 & 0.5038 \\
\hline $97.5 \%$ & 9286 & 0.9068 & 0.880 & 0.7615 & 0.7172 & 0.4847 & 8060 & & 0.66 & 0.842 & 0.7245 & 0.4420 & & & & 0.6829 & & \\
\hline std & 0.2398 & 0.3082 & 0.2699 & 0.0333 & 0.0322 & 0.0817 & 0.0333 & 0.0516 & 0.1091 & 0.0475 & 0.0434 & $0.071 \varepsilon$ & 0.0512 & 0.0390 & 0.1543 & 0.0384 & 0.0473 & 0.0776 \\
\hline
\end{tabular}

Poincare -- Total Power

\begin{tabular}{|c|c|c|c|c|c|c|c|c|c|c|c|c|c|c|c|c|c|c|}
\hline Stat & \multicolumn{3}{|c|}{ individual } & \multicolumn{3}{|c|}{ Bootstrap-All Infants } & \multicolumn{3}{|c|}{ Bootstrap-ALTE } & \multicolumn{3}{|c|}{ Bootstrap-Preterm } & \multicolumn{3}{|c|}{ Bootstrap-Siblings } & \multicolumn{3}{|c|}{ Bootstrap-Healthy } \\
\hline & QS & REM & AWK & QS & REM & AWK & QS & REM & AWK & $\mathrm{QS}$ & REM & AWK & $\mathrm{QS}$ & REM & AWK & QS & REM & AWK \\
\hline $2.5 \%$ & 0.1599 & -0.2455 & -0.0977 & 0.7306 & 0.6102 & 0.2194 & 0.7525 & 0.3904 & 0.2659 & 0.7098 & 0.5782 & 0.1965 & 0.6653 & 0.6352 & 0.1685 & 0.6756 & 0.6518 & 0.3145 \\
\hline $25.0 \%$ & 0.5719 & 0.2770 & 0.1588 & 0.7650 & 0.6513 & 0.2988 & 0.7765 & 0.4591 & 0.3996 & 0.7676 & 0.6298 & 0.2635 & 0.7223 & 0.6811 & 0.3170 & 0.7167 & 0.7134 & 0.4110 \\
\hline $50.0 \%$ & 0.7562 & 0.4817 & 0.3564 & 0.7856 & 0.6729 & 0.3755 & 0.7917 & 0.4955 & 0.4915 & 0.8005 & 0.6572 & 0.3153 & 0.7545 & 0.7052 & 0.5002 & 0.7370 & 0.7465 & 0.4639 \\
\hline $75.0 \%$ & 0.8359 & 0.6714 & 0.5894 & 0.8039 & 0.6924 & 0.4365 & 0.8068 & 0.5279 & 0.6002 & 0.8303 & 0.6840 & 0.3636 & 0.7880 & 0.7298 & 0.5745 & 0.7587 & 0.7738 & 0.5168 \\
\hline $97.5 \%$ & 0.9396 & 0.9196 & 0.8834 & 0.8374 & 0.7232 & 0.4889 & 0.8315 & 0.6049 & 0.6652 & 0.8739 & 0.7305 & 0.4789 & 0.8293 & 0.7717 & 0.6624 & 0.7945 & 0.8203 & 0.6153 \\
\hline std & 0.2093 & 0.3011 & 0.2636 & 0.0283 & 0.0295 & 0.0797 & 0.0212 & 0.0529 & 0.1158 & 0.0433 & 0.0393 & 0.0719 & 0.0436 & 0.0354 & 0.1549 & 0.0305 & 0.0437 & 0.0787 \\
\hline
\end{tabular}

Poincare -- LF/HF

\begin{tabular}{|c|c|c|c|c|c|c|c|c|c|c|c|c|c|c|c|c|c|c|}
\hline Stat & \multicolumn{3}{|c|}{ individual } & \multicolumn{3}{|c|}{ Bootstrap-All Infants } & \multicolumn{3}{|c|}{ Bootstrap-ALTE } & \multicolumn{3}{|c|}{ Bootstrap-Preterm } & \multicolumn{3}{|c|}{ Bootstrap-Siblings } & \multicolumn{3}{|c|}{ Bootstrap-Healthy } \\
\hline & QS & REM & AWK & QS & REM & AWK & QS & REM & AWK & QS & REM & AWK & QS & REM & AWK & QS & REM & AWK \\
\hline $2.5 \%$ & -0.6559 & -0.5771 & -0.3234 & -0.1580 & -0.1041 & -0.0006 & -0.1705 & -0.0816 & -0.0661 & -0.1507 & -0.1141 & -0.0445 & -0.0451 & -0.1871 & -0.0011 & 0.0363 & -0.2034 & -0.1124 \\
\hline $25.0 \%$ & -0.0676 & -0.2323 & -0.0990 & -0.1227 & -0.0703 & 0.0494 & -0.1310 & -0.0152 & 0.0054 & -0.1137 & -0.0716 & -0.0053 & 0.0051 & -0.0963 & 0.1025 & 0.0946 & -0.1117 & -0.0365 \\
\hline $50.0 \%$ & 0.3111 & 0.0454 & 0.0645 & -0.1016 & -0.0524 & 0.0893 & -0.1099 & 0.0178 & 0.0540 & -0.0961 & -0.0485 & 0.0257 & 0.0309 & -0.0552 & 0.1734 & 0.1259 & -0.0606 & 0.0075 \\
\hline $75.0 \%$ & 0.5417 & 0.3815 & 0.2175 & -0.0766 & -0.0330 & 0.1669 & -0.0883 & 0.0511 & 0.5778 & -0.0781 & -0.0273 & 0.1351 & 0.0561 & -0.0223 & 0.2305 & 0.1655 & -0.0071 & 0.0612 \\
\hline $97.5 \%$ & 0.7809 & 0.6928 & 0.7494 & -0.0300 & 0.0122 & 0.4335 & -0.0541 & 0.1224 & 0.6486 & -0.0423 & 0.0207 & 0.1893 & 0.1127 & 0.0302 & 0.3099 & 0.2146 & 0.0804 & 0.1760 \\
\hline std & 0.3791 & 0.3620 & 0.2497 & 0.0332 & 0.0287 & 0.1436 & 0.0304 & 0.0507 & 0.2766 & 0.0273 & 0.0340 & 0.0749 & 0.0402 & 0.0570 & 0.0849 & 0.0467 & 0.0745 & 0.0737 \\
\hline
\end{tabular}


Table 5.6 Correlation between nonlinear HRV parameters and others (cont.)

Poincare -- RMSSD

\begin{tabular}{|c|c|c|c|c|c|c|c|c|c|c|c|c|c|c|c|c|c|c|}
\hline Stat & \multicolumn{3}{|c|}{ individual } & \multicolumn{3}{|c|}{ Bootstrap-All Infants } & \multicolumn{3}{|c|}{ Bootstrap-ALTE } & \multicolumn{3}{|c|}{ Bootstrap-Preterm } & \multicolumn{3}{|c|}{ Bootstrap-Siblings } & \multicolumn{3}{|c|}{ Bootstrap-Healthy } \\
\hline & QS & REM & AWK & QS & REM & AWK & QS & REM & AWK & QS & REM & AWK & $\mathrm{QS}$ & REM & AWK & QS & REM & AWK \\
\hline $2.5 \%$ & 0.2085 & -0.2310 & -0.1553 & 0.7902 & 0.6276 & 0.3559 & 0.7912 & 0.3291 & 0.2223 & 0.8077 & 0.6386 & 0.3952 & 0.7580 & 0.6415 & 0.3082 & 0.6551 & 0.6560 & 0.2694 \\
\hline $25.0 \%$ & 0.6238 & 0.3489 & 0.2342 & 0.8236 & 0.6515 & 0.4072 & 0.8138 & 0.3856 & 0.3420 & 0.8288 & 0.6651 & 0.4377 & 0.7972 & 0.6874 & 0.4096 & 0.7022 & 0.7140 & 0.3581 \\
\hline $50.0 \%$ & 0.7686 & 0.5503 & 0.4330 & 0.8394 & 0.6640 & 0.4345 & 0.8324 & 0.4160 & 0.4137 & 0.8395 & 0.6792 & 0.4608 & 0.8187 & 0.7138 & 0.4610 & 0.7298 & 0.7397 & 0.4075 \\
\hline $75.0 \%$ & 0.8671 & 0.7494 & 0.6178 & 0.8527 & 0.6757 & 0.4716 & 0.8469 & 0.4489 & 0.5710 & 0.8495 & 0.6922 & 0.4846 & 0.8392 & 0.7384 & 0.5085 & 0.7611 & 0.7656 & 0.4540 \\
\hline $97.5 \%$ & 0.9628 & 0.9455 & 0.9046 & 0.8752 & 0.6988 & 0.5386 & 0.8702 & 0.5140 & 0.6390 & 0.8686 & 0.7193 & 0.5309 & 0.8702 & 0.7830 & 0.6035 & 0.8088 & 0.8041 & 0.5329 \\
\hline std & 0.1903 & 0.2988 & 0.2722 & 0.0214 & 0.0182 & 0.0473 & 0.0216 & 0.0470 & 0.1260 & 0.0155 & 0.0204 & 0.0347 & 0.0297 & 0.0361 & 0.0737 & 0.0418 & 0.0390 & 0.0694 \\
\hline
\end{tabular}

Poincare -- SDNN

\begin{tabular}{|c|c|c|c|c|c|c|c|c|c|c|c|c|c|c|c|c|c|c|}
\hline Stat & \multicolumn{3}{|c|}{ individual } & \multicolumn{3}{|c|}{ Bootstrap-All Infants } & \multicolumn{3}{|c|}{ Bootstrap-ALTE } & \multicolumn{3}{|c|}{ Bootstrap-Preterm } & \multicolumn{3}{|c|}{ Bootstrap-Siblings } & \multicolumn{3}{|c|}{ Bootstrap-Healthy } \\
\hline & $\mathrm{QS}$ & REM & AWK & QS & REM & AWK & QS & REM & AWK & QS & REM & AWK & QS & REM & AWK & QS & REM & $\mathrm{AWK}$ \\
\hline $2.5 \%$ & 0.5735 & 0.4577 & 0.5776 & 0.9143 & 0.8929 & 0.7607 & 0.8942 & 0.8424 & 0.7905 & 0.9115 & 0.8904 & 0.7682 & 0.8985 & 0.8902 & 0.6569 & 0.8599 & 0.8802 & 0.8888 \\
\hline $25.0 \%$ & 0.8431 & 0.7478 & 0.8426 & 0.9231 & 0.9007 & 0.7847 & 0.9050 & 0.8623 & 0.8149 & 0.9249 & 0.9018 & 0.7986 & 0.9151 & 0.9036 & 0.7113 & 0.8750 & 0.8964 & 0.9091 \\
\hline $50.0 \%$ & 0.8993 & 0.8512 & 0.8916 & 0.9281 & 0.9047 & 0.8249 & 0.9108 & 0.8717 & 0.8657 & 0.9321 & 0.9075 & 0.8301 & 0.9228 & 0.9105 & 0.8549 & 0.8830 & 0.9049 & 0.9203 \\
\hline $75.0 \%$ & 0.9441 & 0.9122 & 0.9291 & 0.9327 & 0.9091 & 0.8687 & 0.9154 & 0.8797 & 0.8981 & 0.9382 & 0.9124 & 0.8512 & 0.9313 & 0.9166 & 0.9127 & 0.8903 & 0.9125 & 0.9284 \\
\hline $97.5 \%$ & 0.9882 & 0.9653 & 0.9653 & 0.9403 & 0.9155 & 0.8982 & 0.9252 & 0.8938 & 0.9263 & 0.9491 & 0.9227 & 0.8791 & 0.9447 & 0.9264 & 0.9361 & 0.9033 & 0.9263 & 0.9427 \\
\hline std & 0.0996 & 0.1391 & 0.1040 & 0.0068 & 0.0059 & 0.0435 & 0.0079 & 0.0133 & 0.0438 & 0.0096 & 0.0082 & 0.0320 & 0.0120 & 0.0095 & 0.0990 & 0.0112 & 0.0118 & 0.0141 \\
\hline
\end{tabular}

Poincare -- IQRNN

\begin{tabular}{|c|c|c|c|c|c|c|c|c|c|c|c|c|c|c|c|c|c|c|}
\hline Stat & \multicolumn{3}{|c|}{ individual } & \multicolumn{3}{|c|}{ Bootstrap-All Infants } & \multicolumn{3}{|c|}{ Bootstrap-ALTE } & \multicolumn{3}{|c|}{ Bootstrap-Preterm } & \multicolumn{3}{|c|}{ Bootstrap-Siblings } & \multicolumn{3}{|c|}{ Bootstrap-Healthy } \\
\hline & QS & REM & AWK & QS & REM & AWK & QS & REM & AWK & QS & REM & AWK & QS & REM & AWK & QS & REM & AWK \\
\hline $2.5 \%$ & 0.8863 & 0.9171 & 0.6665 & 0.9805 & 0.9843 & 0.8603 & 0.9683 & 0.9705 & 0.6902 & 0.9810 & 0.9862 & 0.9372 & 0.9745 & 0.9802 & 0.9337 & 0.9711 & 0.9786 & 0.9781 \\
\hline $25.0 \%$ & 0.9606 & 0.9606 & 0.9658 & 0.9826 & 0.9860 & 0.9555 & 0.9714 & 0.9764 & 0.7622 & 0.9847 & 0.9883 & 0.9508 & 0.9802 & 0.9825 & 0.9532 & 0.9753 & 0.9837 & 0.9841 \\
\hline $50.0 \%$ & 0.9768 & 0.9788 & 0.9794 & 0.9838 & 0.9868 & 0.9744 & 0.9728 & 0.9788 & 0.9725 & 0.9861 & 0.9891 & 0.9744 & 0.9824 & 0.9838 & 0.9729 & 0.9772 & 0.9858 & 0.9870 \\
\hline $75.0 \%$ & 0.9863 & 0.9875 & 0.9880 & 0.9848 & 0.9876 & 0.9786 & 0.9742 & 0.9808 & 0.9784 & 0.9875 & 0.9899 & 0.9780 & 0.9844 & 0.9849 & 0.9783 & 0.9792 & 0.9874 & 0.9894 \\
\hline $97.5 \%$ & 0.9966 & 0.9959 & 0.9968 & 0.9868 & 0.9888 & 0.9834 & 0.9768 & 0.9838 & 0.9856 & 0.9892 & 0.9912 & 0.9826 & 0.9872 & 0.9871 & 0.9848 & 0.9823 & 0.9903 & 0.9928 \\
\hline std & 0.0374 & 0.0271 & 0.0879 & 0.0017 & 0.0012 & 0.0411 & 0.0021 & 0.0033 & 0.1137 & 0.0021 & 0.0012 & 0.0149 & 0.0031 & 0.0018 & 0.0157 & 0.0029 & 0.0029 & 0.0038 \\
\hline
\end{tabular}

Poincare -- Median

\begin{tabular}{|c|c|c|c|c|c|c|c|c|c|c|c|c|c|c|c|c|c|c|}
\hline Stat & \multicolumn{3}{|c|}{ individual } & \multicolumn{3}{|c|}{ Bootstrap-All Infants } & \multicolumn{3}{|c|}{ Bootstrap-ALTE } & \multicolumn{3}{|c|}{ Bootstrap-Preterm } & \multicolumn{3}{|c|}{ Bootstrap-Siblings } & \multicolumn{3}{|c|}{ Bootstrap-Healthy } \\
\hline & QS & REM & AWK & QS & REM & AWK & QS & REM & AWK & QS & REM & AWK & QS & REM & AWK & QS & REM & AWK \\
\hline $2.5 \%$ & -0.3968 & -0.6931 & -0.5605 & 0.6330 & 0.4484 & 0.1793 & 0.6131 & 0.2405 & -0.0237 & 0.5903 & 0.5567 & 0.1769 & 0.4770 & 0.3392 & 0.1094 & 0.3671 & 0.3484 & 0.1266 \\
\hline $25.0 \%$ & 0.1665 & -0.0985 & -0.0988 & 0.6599 & 0.4780 & 0.2347 & 0.6409 & 0.3106 & 0.0576 & 0.6257 & 0.5919 & 0.2240 & 0.5316 & 0.3937 & 0.1972 & 0.4162 & 0.4635 & 0.2141 \\
\hline $50.0 \%$ & 0.3889 & 0.2010 & 0.1392 & 0.6744 & 0.4921 & 0.2646 & 0.6560 & 0.3477 & 0.1278 & 0.6427 & 0.6083 & 0.2474 & 0.5599 & 0.4216 & 0.2481 & 0.4462 & 0.5426 & 0.2625 \\
\hline $75.0 \%$ & 0.5858 & 0.5377 & 0.4444 & 0.6877 & 0.5069 & 0.2916 & 0.6707 & 0.3856 & 0.1814 & 0.6588 & 0.6253 & 0.2712 & 0.5903 & 0.4588 & 0.2938 & 0.4741 & 0.5891 & 0.3034 \\
\hline $97.5 \%$ & 0.8681 & 0.9609 & 0.7065 & 0.7125 & 0.5323 & 0.3346 & 0.6979 & 0.4590 & 0.2429 & 0.6907 & 0.6535 & 0.3172 & 0.6358 & 0.5157 & 0.3934 & 0.5240 & 0.6528 & 0.3975 \\
\hline std & 0.3247 & 0.4165 & 0.3445 & 0.0203 & 0.0214 & 0.0406 & 0.0217 & 0.0562 & 0.0773 & 0.0254 & 0.0243 & 0.0363 & 0.0421 & 0.0468 & 0.0710 & 0.0408 & 0.0827 & 0.0678 \\
\hline
\end{tabular}


Table 5.6 Correlation between nonlinear HRV parameters and others (cont.)

\begin{tabular}{|c|c|c|c|c|c|c|c|c|c|c|c|c|c|c|c|c|c|c|}
\hline \multicolumn{19}{|c|}{ FD -- HF } \\
\hline Stat & \multicolumn{3}{|c|}{ individual } & \multicolumn{3}{|c|}{ Bootstrap-All Infants } & \multicolumn{3}{|c|}{ Bootstrap-ALTE } & \multicolumn{3}{|c|}{ Bootstrap-Preterm } & \multicolumn{3}{|c|}{ Bootstrap-Siblings } & \multicolumn{3}{|c|}{ Bootstrap-Healthy } \\
\hline & $\mathrm{QS}$ & REM & AWK & QS & REM & AWK & $\mathrm{QS}$ & REM & AWK & QS & REM & AWK & QS & REM & AWK & QS & REM & AWK \\
\hline $2.5 \%$ & 0.2451 & 0.0332 & 0.0998 & 0.7111 & 0.7111 & 0.2850 & 0.7726 & 0.6964 & 0.5475 & 0.6732 & 0.7182 & 0.4337 & 0.7110 & 0.7528 & 0.1193 & 0.8123 & 0.7578 & 0.5799 \\
\hline $25.0 \%$ & 0.6808 & 0.5780 & 0.5108 & 0.7383 & 0.7270 & 0.5277 & 0.7841 & 0.7292 & 0.5843 & 0.7016 & 0.7369 & 0.5087 & 0.7413 & 0.7704 & 0.5239 & 0.8280 & 0.7928 & 0.6475 \\
\hline $50.0 \%$ & 0.8363 & 0.7098 & 0.6349 & 0.7522 & 0.7354 & 0.5755 & 0.7911 & 0.7456 & 0.6035 & 0.7236 & 0.7485 & 0.5527 & 0.7594 & 0.7807 & 0.6075 & 0.8387 & 0.8144 & 0.6827 \\
\hline $75.0 \%$ & 0.9050 & 0.8393 & 0.7754 & 0.7655 & 0.7436 & 0.6095 & 0.7974 & 0.7619 & 0.6240 & 0.7583 & 0.7591 & 0.6046 & 0.7767 & 0.7900 & 0.6658 & 0.8501 & 0.8342 & 0.7140 \\
\hline $97.5 \%$ & 0.9906 & 0.9573 & 0.9084 & 0.7868 & 0.7596 & 0.6498 & 0.8100 & 0.7911 & 0.6691 & 0.7964 & 0.7774 & 0.6519 & 0.8068 & 0.8080 & 0.7366 & 0.8697 & 0.8633 & 0.7707 \\
\hline std & 0.1790 & 0.2138 & 0.2057 & 0.0195 & 0.0122 & 0.0992 & 0.0096 & 0.0245 & 0.0306 & 0.0346 & 0.0155 & 0.0600 & 0.0247 & 0.0146 & 0.1649 & 0.0153 & 0.0284 & 0.0489 \\
\hline
\end{tabular}

\begin{tabular}{|c|c|c|c|c|c|c|c|c|c|c|c|c|c|c|c|c|c|c|}
\hline \multicolumn{19}{|c|}{ FD -- LF } \\
\hline Stat & \multicolumn{3}{|c|}{ individual } & \multicolumn{3}{|c|}{ Bootstrap-All Infants } & \multicolumn{3}{|c|}{ Bootstrap-ALTE } & \multicolumn{3}{|c|}{ Bootstrap-Preterm } & \multicolumn{3}{|c|}{ Bootstrap-Siblings } & \multicolumn{3}{|c|}{ Bootstrap-Healthy } \\
\hline & $\mathrm{QS}$ & REM & AWK & $\mathrm{QS}$ & REM & AWK & QS & REM & AWK & QS & REM & AWK & QS & REM & AWK & QS & REM & AWK \\
\hline $2.5 \%$ & -0.2550 & -0.3991 & -0.4155 & 0.3450 & 0.4421 & 0.0535 & 0.2515 & 0.2038 & 0.0728 & 0.3542 & 0.4091 & 0.0492 & 0.2833 & 0.5571 & 0.0754 & 0.2107 & 0.4490 & 0.1250 \\
\hline $25.0 \%$ & 0.1684 & 0.2413 & 0.0016 & 0.3778 & 0.4691 & 0.0886 & 0.2913 & 0.2708 & 0.0847 & 0.3967 & 0.4460 & 0.1064 & 0.3447 & 0.6012 & 0.1856 & 0.2614 & 0.5039 & 0.2243 \\
\hline $50.0 \%$ & 0.3559 & 0.4207 & 0.2403 & 0.3948 & 0.4832 & 0.1703 & 0.3135 & 0.3058 & 0.2038 & 0.4220 & 0.4625 & 0.1583 & 0.3704 & 0.6225 & 0.2703 & 0.2833 & 0.5288 & 0.2739 \\
\hline $75.0 \%$ & 0.5674 & 0.6022 & 0.4581 & 0.4127 & 0.4979 & 0.2167 & 0.3345 & 0.3421 & 0.3067 & 0.4491 & 0.4778 & 0.1968 & 0.3950 & 0.6435 & 0.3385 & 0.3058 & 0.5543 & 0.3238 \\
\hline $97.5 \%$ & 0.8348 & 0.8410 & 0.8640 & 0.4449 & 0.5268 & 0.2859 & 0.3703 & 0.4042 & 0.4035 & 0.4921 & 0.5115 & 0.2542 & 0.4363 & 0.6813 & 0.4649 & 0.3455 & 0.6016 & 0.4089 \\
\hline std & 0.2915 & 0.3018 & 0.3227 & 0.0257 & 0.0222 & 0.0729 & 0.0310 & 0.0518 & 0.1132 & 0.0363 & 0.0249 & 0.0578 & 0.0389 & 0.0320 & 0.1061 & 0.0335 & 0.0388 & 0.0725 \\
\hline
\end{tabular}

FD -- Total Power

\begin{tabular}{|c|c|c|c|c|c|c|c|c|c|c|c|c|c|c|c|c|c|c|}
\hline Stat & \multicolumn{3}{|c|}{ individual } & \multicolumn{3}{|c|}{ Bootstrap-All Infants } & \multicolumn{3}{|c|}{ Bootstrap-ALTE } & \multicolumn{3}{|c|}{ Bootstrap-Preterm } & \multicolumn{3}{|c|}{ Bootstrap-Siblings } & \multicolumn{3}{|c|}{ Bootstrap-Healthy } \\
\hline & QS & REM & AWK & QS & REM & AWK & QS & REM & AWK & QS & REM & AWK & QS & REM & AWK & QS & REM & AWK \\
\hline $2.5 \%$ & -0.1747 & -0.2275 & -0.3656 & 0.4888 & 0.4951 & 0.0640 & 0.5112 & 0.3050 & 0.0765 & 0.4341 & 0.4457 & 0.0825 & 0.4363 & 0.6018 & 0.0913 & 0.4600 & 0.5155 & 0.1917 \\
\hline $25.0 \%$ & 0.3281 & 0.2767 & 0.0800 & 0.5224 & 0.5176 & 0.1219 & 0.5387 & 0.3670 & 0.0890 & 0.4748 & 0.4825 & 0.1545 & 0.4725 & 0.6408 & 0.2325 & 0.4991 & 0.5722 & 0.2903 \\
\hline $50.0 \%$ & 0.5407 & 0.4585 & 0.2955 & 0.5388 & 0.5309 & 0.2113 & 0.5547 & 0.3956 & 0.2875 & 0.4994 & 0.5012 & 0.2073 & 0.4926 & 0.6607 & 0.3148 & 0.5174 & 0.5961 & 0.3407 \\
\hline $75.0 \%$ & 0.6758 & 0.6269 & 0.5060 & 0.5552 & 0.5452 & 0.2693 & 0.5704 & 0.4263 & 0.3716 & 0.5230 & 0.5186 & 0.2469 & 0.5130 & 0.6793 & 0.3903 & 0.5378 & 0.6194 & 0.3904 \\
\hline $97.5 \%$ & 0.8691 & 0.8770 & 0.8736 & 0.5895 & 0.5717 & 0.3380 & 0.6017 & 0.4860 & 0.4593 & 0.5634 & 0.5513 & 0.3212 & 0.5476 & 0.7127 & 0.5024 & 0.5750 & 0.6609 & 0.4724 \\
\hline std & 0.2558 & 0.2753 & 0.3134 & 0.0249 & 0.0200 & 0.0867 & 0.0235 & 0.0459 & 0.1350 & 0.0349 & 0.0269 & 0.0655 & 0.0296 & 0.0283 & 0.1117 & 0.0293 & 0.0359 & 0.0717 \\
\hline
\end{tabular}

FD - LF/HF

\begin{tabular}{|c|c|c|c|c|c|c|c|c|c|c|c|c|c|c|c|c|c|c|}
\hline Stat & \multicolumn{3}{|c|}{ individual } & \multicolumn{3}{|c|}{ Bootstrap-All Infants } & \multicolumn{3}{|c|}{ Bootstrap-ALTE } & \multicolumn{3}{|c|}{ Bootstrap-Preterm } & \multicolumn{3}{|c|}{ Bootstrap-Siblings } & \multicolumn{3}{|c|}{ Bootstrap-Healthy } \\
\hline & QS & REM & AWK & QS & REM & AWK & QS & REM & AWK & QS & REM & AWK & QS & REM & AWK & QS & REM & AWK \\
\hline $2.5 \%$ & -0.7930 & -0.8190 & -0.7572 & -0.4470 & -0.4801 & -0.4305 & -0.4689 & -0.5911 & -0.5411 & -0.4364 & $4-0.4428$ & $3-0.4098$ & -0.4216 & -0.5607 & -0.4895 & -0.4135 & -0.5834 & -0.5290 \\
\hline $25.0 \%$ & -0.4158 & -0.4896 & -0.5087 & -0.4159 & -0.4582 & -0.3859 & -0.4285 & -0.5503 & -0.4813 & -0.4027 & $7-0.4126$ & -0.3733 & -0.3728 & -0.5304 & -0.4027 & -0.3817 & -0.5340 & -0.4679 \\
\hline $50.0 \%$ & -0.1389 & -0.3322 & -0.3698 & -0.3961 & -0.4457 & -0.3400 & -0.4105 & -0.5278 & -0.4360 & -0.3753 & $3-0.3945$ & -0.3468 & -0.3509 & -0.5135 & -0.3329 & -0.3672 & -0.5025 & -0.4332 \\
\hline $75.0 \%$ & 0.1138 & -0.0661 & -0.1735 & -0.3793 & -0.4308 & -0.2692 & -0.3939 & -0.5060 & 0.0363 & -0.3555 & -0.3755 & -0.2719 & -0.3226 & -0.4934 & -0.2230 & -0.3511 & -0.4710 & -0.3893 \\
\hline $97.5 \%$ & 0.4193 & 0.4950 & 0.3897 & -0.3524 & -0.4030 & 0.0042 & -0.3696 & -0.4617 & 0.0499 & -0.3250 & -0.3350 & -0.2308 & -0.2752 & -0.4625 & -0.1404 & -0.3232 & -0.4142 & -0.3149 \\
\hline std & 0.3411 & 0.3346 & 0.2920 & 0.0249 & 0.0199 & 0.1516 & 0.0255 & 0.0338 & 0.2410 & 0.0306 & 0.0273 & 0.0553 & 0.0373 & 0.0260 & 0.1028 & 0.0231 & 0.0448 & 0.0562 \\
\hline
\end{tabular}


Table 5.6 Correlation between nonlinear HRV parameters and others (cont.)

\begin{tabular}{|c|c|c|c|c|c|c|c|c|c|c|c|c|c|c|c|c|c|c|}
\hline \multicolumn{19}{|c|}{ FD -- RMSSD } \\
\hline Stat & \multicolumn{3}{|c|}{ individual } & \multicolumn{3}{|c|}{ Bootstrap-All Infants } & \multicolumn{3}{|c|}{ Bootstrap-ALTE } & \multicolumn{3}{|c|}{ Bootstrap-Preterm } & \multicolumn{3}{|c|}{ Bootstrap-Siblings } & \multicolumn{3}{|c|}{ Bootstrap-Healthy } \\
\hline & $\overline{\mathrm{QS}}$ & REM & AWK & QS & REM & AWK & $\overline{\mathrm{QS}}$ & REM & AWK & QS & REM & AWK & QS & REM & AWK & QS & REM & AWK \\
\hline $2.5 \%$ & 0.7156 & 0.8046 & 0.6047 & 0.9272 & 0.9088 & 0.8041 & 0.9305 & 0.9068 & 0.7253 & 0.9253 & 0.9133 & 0.8389 & 0.9060 & 0.8857 & 0.6931 & 0.9654 & 0.9534 & 0.8789 \\
\hline $25.0 \%$ & 0.9163 & 0.9006 & 0.8312 & 0.9321 & 0.9145 & 0.8459 & 0.9346 & 0.9160 & 0.7588 & 0.9311 & 0.9188 & 0.8557 & 0.9141 & 0.8989 & 0.7755 & 0.9677 & 0.9597 & 0.9021 \\
\hline $50.0 \%$ & 0.9576 & 0.9439 & 0.9068 & 0.9347 & 0.9170 & 0.8617 & 0.9370 & 0.9205 & 0.8734 & 0.9347 & 0.9218 & 0.8635 & 0.9184 & 0.9046 & 0.8298 & 0.9688 & 0.9627 & 0.9139 \\
\hline $75.0 \%$ & 0.9834 & 0.9769 & 0.9436 & 0.9374 & 0.9199 & 0.8710 & 0.9392 & 0.9251 & 0.8890 & 0.9382 & 0.9248 & 0.8713 & 0.9229 & 0.9100 & 0.8735 & 0.9701 & 0.9657 & 0.9242 \\
\hline $97.5 \%$ & 0.9965 & 0.9948 & 0.9810 & 0.9418 & 0.9248 & 0.8842 & 0.9433 & 0.9331 & 0.9068 & 0.9442 & 0.9310 & 0.8846 & 0.9306 & 0.9191 & 0.9086 & 0.9722 & 0.9709 & 0.9409 \\
\hline std & 0.0732 & 0.0539 & 0.1001 & 0.0038 & 0.0040 & 0.0219 & 0.0034 & 0.0067 & 0.0656 & 0.0049 & 0.0047 & 0.0115 & 0.0063 & 0.0083 & 0.0604 & 0.0018 & 0.0045 & 0.0162 \\
\hline
\end{tabular}

FD -- SDNN

\begin{tabular}{|c|c|c|c|c|c|c|c|c|c|c|c|c|c|c|c|c|c|c|}
\hline Stat & \multicolumn{3}{|c|}{ individual } & \multicolumn{3}{|c|}{ Bootstrap-All Infants } & \multicolumn{3}{|c|}{ Bootstrap-ALTE } & \multicolumn{3}{|c|}{ Bootstrap-Preterm } & \multicolumn{3}{|c|}{ Bootstrap-Siblings } & \multicolumn{3}{|c|}{ Bootstrap-Healthy } \\
\hline & QS & REM & AWK & QS & REM & AWK & QS & REM & AWK & QS & REM & AWK & QS & REM & AWK & QS & REM & AWK \\
\hline $2.5 \%$ & -0.1869 & -0.4962 & -0.2141 & 0.5973 & 0.5608 & 0.2094 & 0.5160 & 0.2653 & 0.1574 & 0.5559 & 0.5459 & 0.2829 & 0.5350 & 0.6383 & 0.1508 & 0.5304 & 0.6643 & 0.1996 \\
\hline $25.0 \%$ & 0.4293 & 0.3997 & 0.2186 & 0.6247 & 0.5856 & 0.2780 & 0.5445 & 0.3356 & 0.2183 & 0.5889 & 0.5705 & 0.3346 & 0.5751 & 0.6793 & 0.2647 & 0.5707 & 0.7037 & 0.3059 \\
\hline $50.0 \%$ & 0.5749 & 0.6307 & 0.4114 & 0.6389 & 0.5978 & 0.3341 & 0.5614 & 0.3710 & 0.3519 & 0.6083 & 0.5858 & 0.3690 & 0.5963 & 0.7005 & 0.3462 & 0.5919 & 0.7222 & 0.3641 \\
\hline $75.0 \%$ & 0.7794 & 0.7873 & 0.5883 & 0.6523 & 0.6094 & 0.3687 & 0.5786 & 0.4035 & 0.4208 & 0.6263 & 0.5983 & 0.3997 & 0.6165 & 0.7195 & 0.4121 & 0.6122 & 0.7404 & 0.4130 \\
\hline $97.5 \%$ & 0.8945 & 0.9249 & 0.8432 & 0.6758 & 0.6313 & 0.4287 & 0.6132 & 0.4640 & 0.5191 & 0.6608 & 0.6284 & 0.4422 & 0.6488 & 0.7535 & 0.5125 & 0.6540 & 0.7744 & 0.5115 \\
\hline std & 0.2682 & 0.3361 & 0.2670 & 0.0201 & 0.0182 & 0.0627 & 0.0249 & 0.0507 & 0.1106 & 0.0271 & 0.0208 & 0.0433 & 0.0305 & 0.0290 & 0.0982 & 0.0310 & 0.0278 & 0.0806 \\
\hline
\end{tabular}

FD -- IQRNN

\begin{tabular}{|c|c|c|c|c|c|c|c|c|c|c|c|c|c|c|c|c|c|c|}
\hline Stat & \multicolumn{3}{|c|}{ individual } & \multicolumn{3}{|c|}{ Bootstrap-All Infants } & \multicolumn{3}{|c|}{ Bootstrap-ALTE } & \multicolumn{3}{|c|}{ Bootstrap-Preterm } & \multicolumn{3}{|c|}{ Bootstrap-Siblings } & \multicolumn{3}{|c|}{ Bootstrap-Healthy } \\
\hline & QS & REM & AWK & QS & REM & AWK & QS & REM & AWK & QS & REM & AWK & QS & REM & AWK & QS & REM & AWK \\
\hline $2.5 \%$ & 0.0336 & -0.4435 & -0.1927 & 0.6269 & 0.4994 & 0.2158 & 0.5993 & 0.1795 & 0.1538 & 0.6279 & 0.4901 & 0.2526 & 0.5567 & 0.5814 & 0.1373 & 0.4916 & 0.5638 & 0.1360 \\
\hline $25.0 \%$ & 0.4916 & 0.3538 & 0.1544 & 626 & 0.5244 & 0.2621 & 6282 & 0.2496 & 0.2587 & 0.6558 & 0.5189 & 0.2943 & 0.6083 & 84 & 556 & 0.5468 & & \\
\hline $50.0 \%$ & 0.6400 & 0.5602 & 0.3339 & 0.6817 & 0.5376 & 0.2888 & 0.6463 & 0.2824 & 0.3177 & 0.6694 & 0.5332 & 0.3140 & 0.6337 & 0.6542 & 0.3108 & 0.5761 & 0.6409 & 0.2957 \\
\hline $75.0 \%$ & 0.7609 & 0.7126 & 0.5151 & 0.6972 & 0.5520 & 0.3129 & 0.6664 & 0.3181 & 0.3757 & 0.6838 & 0.5502 & 0.3348 & 0.6563 & 0.6807 & 0.3704 & 0.6046 & 653 & 0.3461 \\
\hline $97.5 \%$ & 0.9274 & 0.9045 & 0.7834 & 0.7234 & 0.5768 & 0.3586 & 0.6966 & 0.3746 & 0.4676 & 0.7100 & 0.5810 & 0.3746 & 0.6956 & 0.7333 & 0.4655 & 0.6589 & 0.7093 & 0.4364 \\
\hline std & 0.2400 & 0.3238 & 0.2643 & 0.0254 & 0.0199 & 0.0371 & 0.0259 & 0.0498 & 0.0826 & 0.0210 & 0.0229 & 0.0312 & 0.0352 & 0.0394 & 0.0841 & 0.0424 & 0.0372 & 0.0757 \\
\hline
\end{tabular}

FD - Median

\begin{tabular}{|c|c|c|c|c|c|c|c|c|c|c|c|c|c|c|c|c|c|c|}
\hline Stat & \multicolumn{2}{|c|}{ individual } & & \multicolumn{3}{|c|}{ Bootstrap-All Infants } & \multicolumn{3}{|c|}{ Bootstrap-ALTE } & \multicolumn{3}{|c|}{ Bootstrap-Preterm } & Bootstrap-Siblings & & & & & \\
\hline & QS & REM & AWK & QS & REM & AWK & QS & REM & AWK & QS & REM & AWK & QS & REM & AWK & QS & REM & AWK \\
\hline $2.5 \%$ & -0.4487 & -0.4053 & -0.6975 & 0.5288 & 0.4150 & -0.0287 & 0.4057 & 0.1981 & -0.1372 & 0.4251 & 0.4724 & -0.0498 & 0.4214 & 0.3346 & -0.1283 & 0.4963 & 0.5129 & 0.0778 \\
\hline $25.0 \%$ & 0.2431 & 0.1437 & -0.3919 & 0.5569 & 0.4403 & 0.0155 & 0.4427 & 0.2535 & -0.0576 & 0.4691 & 0.5041 & -0.0072 & 0.4722 & 0.3876 & -0.0175 & 0.5310 & 0.5729 & 0.2034 \\
\hline $50.0 \%$ & 0.5154 & 0.4098 & -0.0804 & 0.5701 & 0.4545 & 0.0397 & 0.4611 & 0.2845 & -0.0183 & 0.4899 & 0.5215 & 0.0144 & 0.4927 & 0.4218 & 0.0449 & 0.5479 & 0.6036 & 0.2598 \\
\hline $75.0 \%$ & 0.7460 & 0.6752 & 0.3164 & 0.5845 & 0.4675 & 0.0612 & 0.4806 & 0.3145 & 0.0204 & 0.5113 & 0.5385 & 0.0335 & 0.5143 & 0.4484 & 0.1071 & 0.5672 & 0.6333 & 0.3159 \\
\hline $97.5 \%$ & 0.9254 & 0.9271 & 0.8465 & 0.6117 & 0.4967 & 0.1048 & 0.5182 & 0.3688 & 0.0992 & 0.5502 & 0.5692 & 0.0723 & 0.5528 & 0.5012 & 0.2236 & 0.5967 & 0.6830 & 0.4194 \\
\hline std & 0.3929 & 0.3789 & 0.4502 & 0.0209 & 0.0208 & 0.0338 & 0.0283 & 0.0444 & 0.0596 & 0.0321 & 0.0253 & 0.0309 & 0.0325 & 0.0430 & 0.0911 & 0.0261 & 0.0437 & 0.0869 \\
\hline
\end{tabular}


Table 5.7 Correlations between other HRV parameters

SDNN -- RMSSD

\begin{tabular}{|c|c|c|c|c|c|c|c|c|c|c|c|c|c|c|c|c|c|c|}
\hline \multirow[t]{2}{*}{ Stat } & \multicolumn{3}{|c|}{ individual } & \multicolumn{3}{|c|}{ Bootstrap-All Infants } & \multicolumn{3}{|c|}{ Bootstrap-ALTE } & \multicolumn{3}{|c|}{ Bootstrap-Preterm } & \multicolumn{3}{|c|}{ Bootstrap-Siblings } & \multicolumn{3}{|c|}{ Bootstrap-Healthy } \\
\hline & QS & REM & & QS & REM & AWK & QS & REM & & QS & REM & AWK & QS & REM & AWK & QS & REM & AWK \\
\hline & 0.0863 & & & 0.7035 & 0.7059 & & & 3645 & & 4 & & & 7 & 24 & & 40 & & \\
\hline $25.0 \%$ & 5257 & 0.4628 & & 0.7278 & 0 . & 0.5246 & 303 & 309 & & 8 & & & 34 & & & 0.6140 & & \\
\hline $50.0 \%$ & 7086 & 0.6701 & & 0.740 & & & 6460 & 612 & & 7 & & & 0.7320 & 098 & & 0.6358 & & 004 \\
\hline $75.0 \%$ & 0.8245 & 0.8070 & & 0.7520 & & 673 & 6620 & 0.4914 & & 0.7627 & & & 0.7542 & 0.8239 & 462 & 0.6559 & & \\
\hline $97.5 \%$ & 0.9527 & 0.9042 & & 0.7735 & 0.7603 & 0.6051 & 0.6915 & 0.5419 & 0.7170 & 0.7901 & 0.7766 & 0.6161 & 0.7877 & 0.8493 & 0.7211 & 0.6984 & 0.8336 & $0.0<10$ \\
\hline std & 0.2203 & 0.2934 & 0.2582 & 0.0178 & 0.0137 & 0.0306 & 0.0228 & 0.0451 & 0.1131 & 0.0235 & 0.0156 & 0.0263 & 0.0338 & 0.0221 & 0.0799 & 0.0313 & 0.0253 & 0.0653 \\
\hline
\end{tabular}

$\begin{array}{lll}-0.139 & 0.9527\end{array}$

\begin{tabular}{|c|c|c|c|c|c|c|c|c|c|c|c|c|c|c|c|c|c|c|}
\hline \multicolumn{19}{|c|}{ SDNN -- IQRSD } \\
\hline Stat & \multicolumn{3}{|c|}{ individual } & \multicolumn{3}{|c|}{ Bootstrap-All Infants } & \multicolumn{3}{|c|}{ Bootstrap-ALTE } & \multicolumn{3}{|c|}{ Bootstrap-Preterm } & \multicolumn{3}{|c|}{ Bootstrap-Siblings } & \multicolumn{3}{|c|}{ Bootstrap-Healthy } \\
\hline & QS & REM & AWK & QS & REM & AWK & QS & REM & AWK & QS & REM & AWK & QS & REM & AWK & QS & REM & AWK \\
\hline $2.5 \%$ & -0.2437 & -0.3403 & -0.2459 & 0.6669 & 0.6563 & 0.1906 & 0.5673 & 0.3280 & 0.0678 & 0.6317 & 0.6449 & 0.3027 & 0.5993 & 0.7450 & 0.1739 & 0.5564 & 0.7018 & 0.2881 \\
\hline $25.0 \%$ & 0.4017 & 0.3385 & 0.2244 & 0.6931 & 0.6803 & 0.3274 & 0.5994 & 0.3967 & 0.1338 & 0.6717 & 0.6701 & 0.3536 & 0.6580 & 0.7778 & 0.2872 & 0.5995 & 0.7459 & 0.3954 \\
\hline $50.0 \%$ & 0.5322 & 0.5783 & 0.4508 & 0.7074 & 0.6906 & 0.3858 & 0.6167 & 0.4304 & 0.3903 & 0.6935 & 0.6819 & 0.3871 & 0.6858 & 0.7938 & 0.3978 & 0.6227 & 0.7645 & 0.4444 \\
\hline $75.0 \%$ & 0.7338 & 0.7315 & 0.6338 & 0.7205 & 0.7010 & 0.4265 & 0.6347 & 0.4636 & 0.4477 & 0.7123 & 0.6941 & 0.4202 & 0.7132 & 0.8089 & 0.4749 & 0.6451 & 0.7826 & 0.4902 \\
\hline $97.5 \%$ & 0.9267 & 0.9250 & 0.8167 & 0.7448 & 0.7193 & 0.4891 & 0.6660 & 0.5238 & 0.5509 & 0.7441 & 0.7170 & 0.4693 & 0.7564 & 0.8348 & 0.5812 & 0.6864 & 0.8131 & 0.5780 \\
\hline std & 0.2622 & 0.3002 & 0.2873 & 0.0203 & 0.0156 & 0.0882 & 0.0255 & 0.0498 & 0.1589 & 0.0287 & 0.0180 & 0.0438 & 0.0406 & 0.0232 & 0.1162 & 0.0332 & 0.0277 & 0.0720 \\
\hline
\end{tabular}

SDNN -- Median

\begin{tabular}{|c|c|c|c|c|c|c|c|c|c|c|c|c|c|c|c|c|c|c|}
\hline \multirow[t]{2}{*}{ Stat } & \multicolumn{3}{|c|}{ individual } & \multicolumn{3}{|c|}{ Bootstrap-All Infants } & \multicolumn{3}{|c|}{ Bootstrap-ALTE } & \multicolumn{3}{|c|}{ Bootstrap-Preterm } & \multicolumn{3}{|c|}{ Bootstrap-Siblings } & \multicolumn{3}{|c|}{ Bootstrap-Healthy } \\
\hline & QS & REM & AWK & QS & REM & $\mathrm{AWK}$ & QS & REM & AWK & QS & REM & $\Delta \mathrm{WK}$ & QS & RFM & AWK & QS & REM & AWK \\
\hline $2.5 \%$ & -0.3791 & -0.4626 & & 0.5714 & 0.5572 & 0.0509 & .5028 & 0.3337 & & & 0.6416 & & 0.4026 & 0.5348 & & 0.2586 & 0.4695 & \\
\hline $25.0 \%$ & 0.1030 & 0.0 & & 0.5980 & 0.5821 & 0.1752 & & 0.3962 & & & 722 & & & & & & & 233 \\
\hline $50.0 \%$ & & & & 0.61 & & & & & & & & & & & & & & 760 \\
\hline $75.0 \%$ & & & & & & & & & & & & & & & & & & \\
\hline $7.5 \%$ & & & & 0.6480 & & & & 0.5070 & & & & & & & & 0.3984 & & \\
\hline std & 0.3102 & 0.3833 & 0.3257 & 0.0191 & 0.0177 & 0.0840 & 0.0236 & 0.0446 & 0.1400 & 0.0277 & 0.0217 & 0.0471 & 0.0463 & 0.0324 & 0.0941 & 0.0368 & 0.0621 & 0.0768 \\
\hline
\end{tabular}

$-0.4760 .9068$ 
Table 5.7 Correlations between other HRV parameters (cont.)

Median -- IQRNN

\begin{tabular}{|c|c|c|c|c|c|c|c|c|c|c|c|c|c|c|c|c|c|c|}
\hline \multirow[t]{2}{*}{ Stat } & \multicolumn{3}{|c|}{ individual } & \multicolumn{3}{|c|}{ Bootstrap-All Infants } & \multicolumn{3}{|c|}{ Bootstrap-ALTE } & \multicolumn{3}{|c|}{ Bootstrap-Preterm } & \multicolumn{3}{|c|}{ Bootstrap-Siblings } & \multicolumn{3}{|c|}{ Bootstrap-Healthy } \\
\hline & QS & REM & AWK & QS & REM & AWK & QS & REM & AWK & QS & REM & AWK & QS & REM & & QS & REM & AWK \\
\hline & -0.3864 & 0620 & $3-0.5448$ & 0 & 0.45 & 0.236 & 5 & 021 & & 6 & 0.55 & & 3 & 1 & & 51 & 0.32 & \\
\hline $25.0 \%$ & 0.1034 & -0.0794 & & 0.6178 & 0.4793 & 0.2803 & 0.6032 & 0.2838 & & 0.5963 & 0.5926 & & 0.47 & 0.4050 & 0.2426 & 0.3409 & & 250 \\
\hline $50.0 \%$ & 0.3587 & 0.2026 & 0.2085 & 0.6333 & 0.4931 & 0.3019 & 0.6196 & 0.3201 & 0.1735 & 0.6130 & 0.6079 & 0.2820 & 0.5096 & 0.4334 & 0.2913 & 0.3715 & 0.4967 & 0.2777 \\
\hline $75.0 \%$ & 0.5796 & 0.5553 & 0.4785 & 0.6479 & 0.5081 & 0.3232 & 0.6348 & 0.3573 & 0.2082 & 0.6285 & 0.6248 & 0.3068 & 0.5434 & 0.4684 & 0.3376 & 0.4035 & & 0.3227 \\
\hline $97.5 \%$ & 0.8705 & 0.9402 & & 0.6741 & 0.5320 & 0.3620 & 0.6666 & 0.4238 & 0.2678 & 0.6575 & 0.6544 & 0.3505 & 0.5974 & 0.5262 & 0.4254 & 0.4581 & 0.6093 & 0.4126 \\
\hline std & 0.3281 & 0.4058 & 0.3490 & 0.0220 & 0.0208 & 0.0322 & 0.0238 & 0.0534 & 0.0516 & 0.0243 & 0.0239 & 0.0354 & 0.0480 & 0.0459 & 0.0699 & 0.0439 & 0.0757 & 0.0706 \\
\hline
\end{tabular}

$-0.630 .9402$

$\min \max$

Median -- RMSSD

\begin{tabular}{|c|c|c|c|c|c|c|c|c|c|c|c|c|c|c|c|c|c|c|}
\hline \multirow[t]{2}{*}{ Stat } & \multicolumn{3}{|c|}{ individual } & \multicolumn{3}{|c|}{ Bootstrap-All Infants } & \multicolumn{3}{|c|}{ Bootstrap-ALTE } & \multicolumn{3}{|c|}{ Bootstrap-Preterm } & \multicolumn{3}{|c|}{ Bootstrap-Siblings } & \multicolumn{3}{|c|}{ Bootstrap-Healthy } \\
\hline & QS & REM & AWK & QS & REM & AWK & QS & REM & AWK & QS & REM & AWK & QS & REM & AWK & QS & REM & AWK \\
\hline $2.5 \%$ & -0.2390 & -0.1148 & $3-0.5568$ & 0.7082 & 0.6464 & 0.2615 & 0.6185 & 0.4707 & 0.0985 & 0.6309 & 0.6745 & 0.2551 & 0.6560 & 0.6478 & 0.1927 & 0.6217 & 0.6821 & 0.3853 \\
\hline $25.0 \%$ & 0.4457 & 0.2580 & -0.1506 & 0.7265 & 0.6620 & 0.3140 & 0.6484 & 0.5153 & 0.1965 & 0.6694 & 0.6984 & 0.2982 & 0.6964 & 0.6768 & 0.2964 & 0.6486 & 0.7229 & 0.4743 \\
\hline $50.0 \%$ & 0.6746 & 0.5792 & 0.2007 & 0.7361 & 0.6703 & 0.3428 & 0.6616 & 0.5373 & 0.3012 & 0.6848 & 0.7096 & 0.3205 & 0.7138 & 0.6925 & 0.3581 & 0.6614 & 0.7434 & .5134 \\
\hline $75.0 \%$ & 0.8317 & 0.7303 & & 0.7443 & 0.6784 & 0.3677 & 0.6748 & 0.5565 & 0.3509 & 0.7007 & 0.7206 & 0.3437 & 0.7289 & 0.7061 & 0.4099 & 0.6755 & 0.7639 & 0.5537 \\
\hline $97.5 \%$ & 0.9374 & 0.9642 & 0.8758 & 0.7619 & 0.6963 & 0.4081 & 0.7005 & 0.5927 & 0.4285 & 0.7294 & 0.7429 & 0.3784 & 0.7578 & 0.7346 & 0.4964 & 0.6984 & 0.7942 & 0.6199 \\
\hline std & 0.3148 & 0.3292 & 0.4244 & 0.0134 & 0.0129 & 0.0387 & 0.0202 & 0.0306 & 0.0941 & 0.0243 & 0.0174 & 0.0321 & 0.0252 & 0.0221 & 0.0779 & 0.0196 & 0.0292 & 0.0593 \\
\hline
\end{tabular}

$-0.5570 .9642$

$\min \max$

Median -- IQRSD

\begin{tabular}{|c|c|c|c|c|c|c|c|c|c|c|c|c|c|c|c|c|c|c|}
\hline \multirow[t]{2}{*}{ Stat } & \multicolumn{3}{|c|}{ individual } & \multicolumn{3}{|c|}{ Bootstrap-All Infants } & \multicolumn{3}{|c|}{ Bootstrap-ALTE } & \multicolumn{3}{|c|}{ Bootstrap-Preterm } & \multicolumn{3}{|c|}{ Bootstrap-Siblings } & \multicolumn{3}{|c|}{ Bootstrap-Healthy } \\
\hline & QS & REM & AWK & QS & REM & $\mathrm{AWK}$ & QS & REM & AWK & QS & REM & AWK & QS & REM & $\mathrm{AWK}$ & QS & REM & AWK \\
\hline $2.5 \%$ & -0.3195 & -0.1828 & -0.4304 & 0.6857 & 0.6285 & 3585 & 0.5670 & 4117 & 0.2521 & .6040 & .6472 & .2657 & 0.6390 & 0.6184 & 0.4443 & 0.6017 & 0.6495 & .3996 \\
\hline $25.0 \%$ & 0.3890 & 0.2478 & 0.1180 & 0.7089 & 0.6457 & 0.3989 & 0.6064 & 0.4615 & 0.3412 & 0.6432 & 0.6727 & 0.3102 & 0.6788 & 0.6521 & 0.5305 & 0.6305 & 0.6946 & 0.4971 \\
\hline $50.0 \%$ & 0.6496 & 0.5836 & 0.4705 & 0.7198 & 0.6552 & 0.4218 & 0.6237 & 0.4884 & 0.3818 & 0.6621 & 0.6851 & 0.3325 & 0.6987 & 0.6700 & 748 & 0.6445 & 0.7157 & 0.5377 \\
\hline $75.0 \%$ & 0.8066 & 0.7816 & 0.7266 & 0.7301 & 0.6650 & 0.4435 & 0.6420 & 0.5138 & 0.4218 & 0.6790 & 0.6976 & 0.3524 & 0.7165 & 0.6857 & 0.6161 & 0.6599 & 0.7371 & 0.5788 \\
\hline $97.5 \%$ & 0.9451 & 0.9244 & 0.9221 & 0.7494 & 0.6826 & 0.4811 & 0.6750 & 0.5587 & 0.4950 & 0.7081 & 0.7183 & 0.3935 & 0.7460 & 0.7172 & 0.6780 & 0.6849 & 0.7732 & 0.6441 \\
\hline std & 0.3464 & 0.3231 & 0.3981 & 0.0159 & 0.0140 & 0.0316 & 0.0269 & 0.0381 & 0.0614 & 0.0265 & 0.0187 & 0.0322 & 0.0278 & 0.0247 & 0.0600 & 0.0213 & 0.0314 & 0.0623 \\
\hline
\end{tabular}

$-0.430 .9451$ 
APPENDIX E

RESULT FOR THE INFANT CLASSIFICATION 


\section{Table E1 Logistic Regression in SAS EM}

\section{The SAS System}

The DMREG Procedure

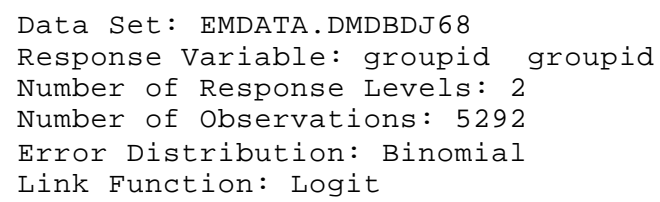

Optimization Start

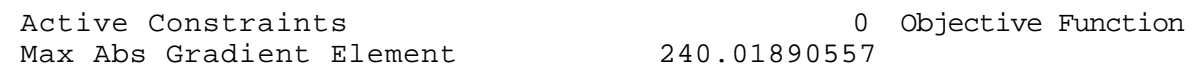

$\begin{array}{rrrr}\text { Iter } & \text { Restarts } & \begin{array}{r}\text { Function } \\ \text { Calls }\end{array} & \begin{array}{r}\text { Active } \\ \text { Constraints }\end{array} \\ 1 & & 2 & 0 \\ 2 & 0 & 3 & 0 \\ 3 & 0 & 4 & 0 \\ 4 & 0 & 5 & \\ 5 & 0 & 6 & \end{array}$

Optimization Results

Iterations

Hessian Calls

Objective Function

Ridge
Objective Function
Objective Max Abs

Function Gradient Change Element and

Predicted Change

\begin{tabular}{|c|c|c|c|c|c|}
\hline 0 & 1904 & 383.5 & 249.6 & 0 & 0.878 \\
\hline 0 & 1847 & 56.2945 & 23.1775 & 0 & 1.073 \\
\hline D & 1846 & 1.4463 & 1.0276 & 0 & 1.034 \\
\hline 0 & 1846 & 0.00427 & 0.00313 & 0 & 1.002 \\
\hline 0 & 1846 & $6.74 \mathrm{E}-8$ & $4.659 \mathrm{E}-8$ & 0 & 1.000 \\
\hline
\end{tabular}

5
$6 \quad$ Function Calls
Active Constraints
0 Actual Over Pred Change

0 Actual Over Pred Change

GCONV convergence criterion satisfied.

Model Fitting Information and Testing Global Null Hypothesis BETA=0

$\begin{array}{lcr}\text { Criterion } & \begin{array}{c}\text { Intercept } \\ \text { Only }\end{array} & \begin{array}{c}\text { and } \\ \text { Covariates }\end{array} \\ \text { AIC } & 4576.003 & 3723.586 \\ \text { SBC } & 4582.576 & 3828.770 \\ -2 \text { LOG L } & 4574.003 & 3691.586\end{array}$

Chi-Square for Covariates

882.416 with 15 DF $\quad(p<.0001)$ 
Type III Analysis of Effects

$\begin{array}{lrrr}\text { Effect } & \text { DF } & \begin{array}{c}\text { Wald } \\ \text { Chi-Square }\end{array} & \begin{array}{c}\text { Pr }> \\ \text { Chi-Square }\end{array} \\ \text { Mean } & 1 & 153.0434 & <.0001 \\ \text { REMratio } & 1 & 208.6064 & <.0001 \\ \text { REMtime } & 1 & 139.5561 & <.0001 \\ \text { SSC1 } & 1 & 36.2615 & <.0001 \\ \text { SSC2 } & 1 & 8.1810 & 0.0042 \\ \text { SDNN_ } & 1 & 1.5584 & 0.2119 \\ \text { RMSSD } & 1 & 46.2569 & <.0001 \\ \text { ApEn } & 1 & 0.0412 & 0.8392 \\ \text { Poincare } & 1 & 0.2224 & 0.6372 \\ \text { FD } & 1 & 23.3718 & 0.0001 \\ \text { SDANN } & 1 & 0.0309 & 0.0251 \\ \text { SDNNIDX } & 1 & 5.0184 & 0.0017 \\ \text { HFW } & 1 & 9.8270 & 0.1472 \\ \text { LFW } & 1 & 2.1013 & 0.0179 \\ \text { LFW_HFW } & 1 & 5.6068 & \end{array}$

Analysis of Maximum Likelihood Estimates

\begin{tabular}{|c|c|c|c|c|c|c|c|c|}
\hline \multirow[b]{2}{*}{ Parameter } & & \multirow[b]{2}{*}{$\mathrm{DF}$} & \multirow[b]{2}{*}{ Estimate } & \multirow{2}{*}{$\begin{array}{c}\text { Standard } \\
\text { Error }\end{array}$} & \multirow{2}{*}{$\begin{array}{c}\text { Wald } \\
\text { Chi-square }\end{array}$} & \multirow{2}{*}{$\begin{array}{c}\text { Pr > } \\
\text { Chi-square }\end{array}$} & \multicolumn{2}{|c|}{ Standardized } \\
\hline & & & & & & & Estimate & $\exp ($ Est) \\
\hline Intercept & & 1 & 15.9335 & 2.8199 & 31.93 & $<.0001$ & $\bullet$ & 999.000 \\
\hline Mean & & 1 & -0.0225 & 0.00182 & 153.04 & $<.0001$ & -0.743713 & 0.978 \\
\hline REMratio & & 1 & 31.0941 & 2.1529 & 208.61 & $<.0001$ & 1.924374 & 999.000 \\
\hline REMt ime & & 1 & -0.00093 & 0.000079 & 139.56 & $<.0001$ & -1.583157 & 0.999 \\
\hline ssc1 & 0 & 1 & 0.6094 & 0.1012 & 36.26 & $<.0001$ & . & 1.839 \\
\hline ssc2 & 0 & 1 & 0.2210 & 0.0773 & 8.18 & 0.0042 & . & 1.247 \\
\hline $\mathrm{SDNN}_{-}$ & & 1 & -0.0131 & 0.0105 & 1.56 & 0.2119 & -0.097972 & 0.987 \\
\hline RMSSD & & 1 & 0.2676 & 0.0393 & 46.26 & $<.0001$ & 0.971635 & 1.307 \\
\hline ApEn & & 1 & -0.0676 & 0.3329 & 0.04 & 0.8392 & -0.009810 & 0.935 \\
\hline Poincare & & 1 & -0.00304 & 0.00644 & 0.22 & 0.6372 & -0.025893 & 0.997 \\
\hline $\mathrm{FD}$ & & 1 & -11.7251 & 2.4253 & 23.37 & $<.0001$ & -0.442293 & 0.000 \\
\hline SDANN & & 1 & -0.00104 & 0.00589 & 0.03 & 0.8605 & -0.006722 & 0.999 \\
\hline SDNNIDX & & 1 & 0.0194 & 0.00866 & 5.02 & 0.0251 & 0.101612 & 1.020 \\
\hline $\mathrm{HFW}$ & & 1 & $-2.43 E-6$ & $7.748 E-7$ & 9.83 & 0.0017 & -0.281090 & 1.000 \\
\hline LEW & & 1 & $1.355 E-7$ & $9.348 E-8$ & 2.10 & 0.1472 & 0.061522 & 1.000 \\
\hline LFW_HFW & & 1 & 0.0222 & 0.00936 & 5.61 & 0.0179 & 0.069252 & 1.022 \\
\hline
\end{tabular}

Odds Ratio Estimates

Input

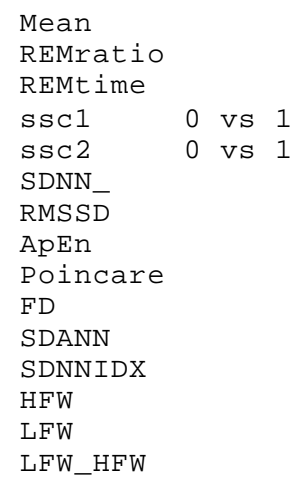

Odds Ratio

0.978

999.000

0.999

3.383

1. 556

0.987

1. 307

0.935

0.997

0.000

0.999

1. 020

1.000

1.000

1. 022 
Step 1. Variable SDANN is removed:

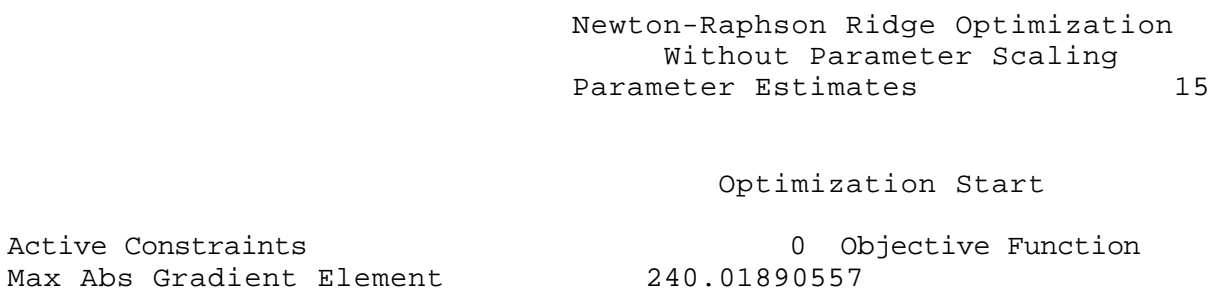

Optimization start

Active Constraints Max Abs Gradient Element

0 Objective Function 240.01890557

2287.0012666

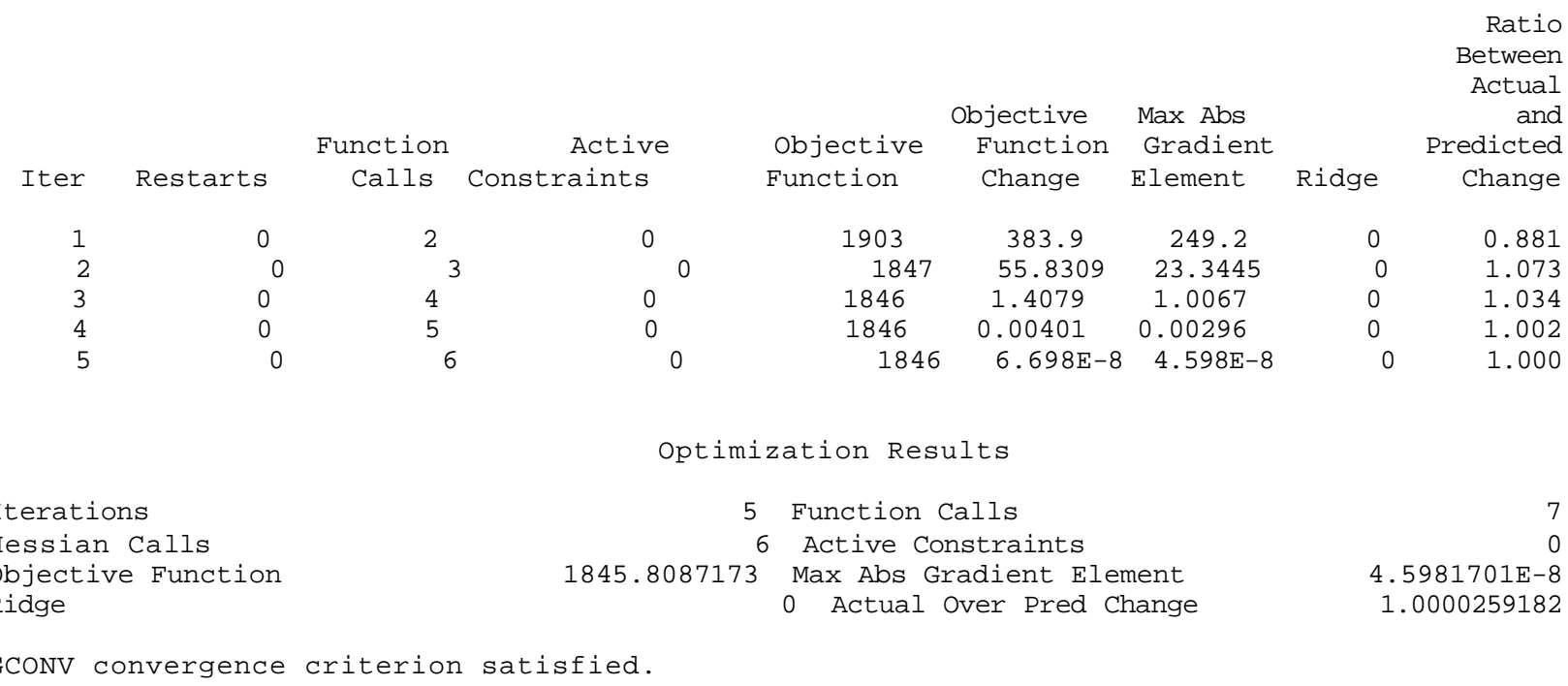

Model Fitting Information and Testing Global Null Hypothesis BETA=0

$\begin{array}{lccc}\text { Intercept } & \begin{array}{c}\text { Intercept } \\ \text { Ond }\end{array} & \begin{array}{c}\text { and } \\ \text { Covariates }\end{array} & \text { Chi-Square for Covariates } \\ \text { AIC } & 4576.003 & 3721.617 & . \\ \text { SBC } & 4582.576 & 3820.227 & . \\ -2 \text { LOG L } & 4574.003 & 3691.617 & 882.385 \text { with } 14 \text { DF }(\mathrm{p}<.0001)\end{array}$

Type II Analysis of Effects

$\begin{array}{lrrr}\text { Effect } & \text { DF } & \begin{array}{c}\text { Wald } \\ \text { Chi-Square }\end{array} & \begin{array}{c}\text { Pr }> \\ \text { Chi-Square }\end{array} \\ \text { Mean } & 1 & 153.6630 & <.0001 \\ \text { REMratio } & 1 & 208.5800 & <.0001 \\ \text { REMtime } & 1 & 139.6798 & <.0001 \\ \text { SSC1 } & 1 & 38.1479 & <.0001 \\ \text { SSC2 } & 1 & 8.4667 & 0.0036 \\ \text { SDNN_ } & 1 & 1.5608 & 0.2116 \\ \text { RMSSD } & 1 & 46.2962 & <.0001 \\ \text { ApEn } & 1 & 0.0402 & 0.8411 \\ \text { Poincare } & 1 & 0.2216 & 0.6378 \\ \text { FD } & 1 & 23.4121 & <.0001 \\ \text { SDNNIDX } & 1 & 5.5666 & 0.0183 \\ \text { HFW } & 1 & 9.8478 & 0.0017 \\ \text { LFW } & 1 & 2.1574 & 0.1419 \\ \text { LFW_HFW } & 1 & 5.6046 & 0.0179\end{array}$


Analysis of Maximum Likelihood Estimates

\begin{tabular}{|c|c|c|c|c|c|c|c|c|}
\hline \multirow[b]{2}{*}{ Parameter } & & & & Standard & $\mathrm{Wald}$ & $\operatorname{Pr}>$ & \multicolumn{2}{|c|}{ Standardized } \\
\hline & & $\mathrm{DF}$ & Estimate & Error & Chi-square & Chi-square & Estimate & $\exp (E s t)$ \\
\hline Intercept & & 1 & 15.9361 & 2.8198 & 31.94 & $<.0001$ & . & 999.000 \\
\hline Mean & & 1 & -0.0225 & 0.00182 & 153.66 & $<.0001$ & -0.744167 & 0.978 \\
\hline REMratio & & 1 & 31.0851 & 2.1524 & 208.58 & $<.0001$ & 1.923814 & 999.000 \\
\hline REMtime & & 1 & -0.00093 & 0.000079 & 139.68 & $<.0001$ & -1.582002 & 0.999 \\
\hline ssc1 & 0 & 1 & 0.6129 & 0.0992 & 38.15 & $<.0001$ & . & 1.846 \\
\hline ssc2 & 0 & 1 & 0.2176 & 0.0748 & 8.47 & 0.0036 & • & 1.243 \\
\hline $\mathrm{SDNN}_{-}$ & & 1 & -0.0131 & 0.0105 & 1.56 & 0.2116 & -0.098040 & 0.987 \\
\hline RMSSD & & 1 & 0.2677 & 0.0393 & 46.30 & $<.0001$ & 0.971936 & 1.307 \\
\hline ApEn & & 1 & -0.0667 & 0.3328 & 0.04 & 0.8411 & -0.009689 & 0.935 \\
\hline Poincare & & 1 & -0.00303 & 0.00644 & 0.22 & 0.6378 & -0.025847 & 0.997 \\
\hline FD & & 1 & -11.7336 & 2.4250 & 23.41 & $<.0001$ & -0.442613 & 0.000 \\
\hline SDNNIDX & & 1 & 0.0188 & 0.00797 & 5.57 & 0.0183 & 0.098493 & 1.019 \\
\hline $\mathrm{HFW}$ & & 1 & $-2.43 E-6$ & $7.748 \mathrm{E}-7$ & 9.85 & 0.0017 & -0.281375 & 1.000 \\
\hline LFW & & 1 & $1.368 E-7$ & $9.316 \mathrm{E}-8$ & 2.16 & 0.1419 & 0.062128 & 1.000 \\
\hline LFW_HFW & & 1 & 0.0222 & 0.00936 & 5.60 & 0.0179 & 0.069192 & 1.022 \\
\hline
\end{tabular}

Odds Ratio Estimates

Input

Mean

REMratio

REMtime

SSC1 0 vs 1

SSC2 0 Vs 1

SDNN-

RMSSD

ApEn

Poincare

FD

SDNNIDX

HFW

LFW

LEW_HEW
Odds Ratio

0.978

999.000

0.999

3.407

1. 545

0.987

1.307

0.935

0.997

0.000

1.019

1.000

1.000

1. 022

Step 2. Variable ApEn is removed:

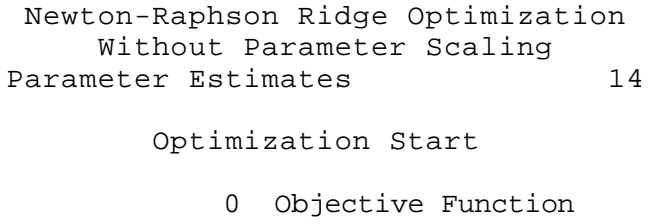

\begin{tabular}{|c|c|c|c|c|c|c|c|c|}
\hline Iter & Restarts & $\begin{array}{l}\text { Function } \\
\text { Calls }\end{array}$ & $\begin{array}{l}\text { Active } \\
\text { Constraints }\end{array}$ & $\begin{array}{l}\text { Objective } \\
\text { Function }\end{array}$ & $\begin{array}{l}\text { Objective } \\
\text { Function } \\
\text { Change }\end{array}$ & $\begin{array}{l}\text { Max Abs } \\
\text { Gradient } \\
\text { Element }\end{array}$ & Ridge & $\begin{array}{r}\text { Ratio } \\
\text { Between } \\
\text { Actual } \\
\text { and } \\
\text { Predicted } \\
\text { Change }\end{array}$ \\
\hline 1 & 0 & 2 & 0 & 1902 & 384.6 & 248.9 & 0 & 0.886 \\
\hline 2 & 0 & 3 & 0 & 1847 & 55.2098 & 23.6136 & 0 & 1.07 \\
\hline 3 & 0 & 4 & 0 & 1846 & 1.3588 & 0.9723 & 0 & 1.0 \\
\hline 4 & 0 & 5 & 0 & 1846 & 0.00376 & 0.00276 & 0 & 1.00 \\
\hline 5 & 0 & 6 & 0 & 1846 & $7.644 \mathrm{E}-8$ & $5.158 \mathrm{E}-8$ & 0 & 1.000 \\
\hline
\end{tabular}


Optimization Results

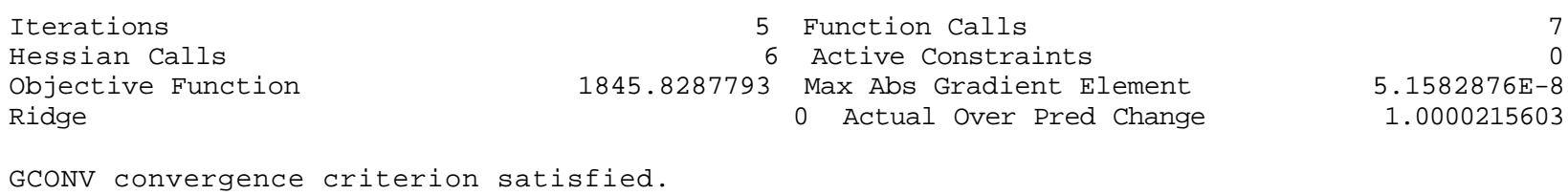

GCONV convergence criterion satisfied.

Model Fitting Information and Testing Global Null Hypothesis BETA=0

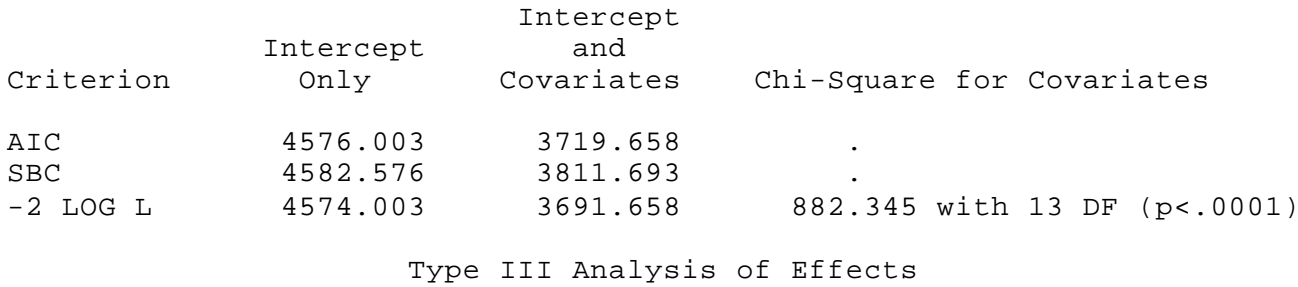

$\begin{array}{lrrr}\text { Effect } & \text { DF } & \begin{array}{c}\text { Wald } \\ \text { Chi-Square }\end{array} & \begin{array}{c}\text { Pr }> \\ \text { Chi-Square }\end{array} \\ \text { Mean } & 1 & 173.3763 & <.0001 \\ \text { REMratio } & 1 & 208.6412 & <.0001 \\ \text { REMtime } & 1 & 139.7086 & <.0001 \\ \text { SSC1 } & 1 & 38.6401 & <.0001 \\ \text { SSC2 } & 1 & 8.4707 & 0.0036 \\ \text { SDNN_ } & 1 & 1.8274 & 0.1764 \\ \text { RMSSD } & 1 & 47.9938 & <.0001 \\ \text { POincare } & 1 & 0.2369 & 0.6265 \\ \text { FD } & 1 & 33.4693 & <.0001 \\ \text { SDNNIDX } & 1 & 5.7703 & 0.0163 \\ \text { HFW } & 1 & 9.8186 & 0.0017 \\ \text { LFW } & 1 & 2.1636 & 0.1413 \\ \text { LFW_HFW } & 1 & 6.2998 & 0.0121\end{array}$

Analysis of Maximum Likelihood Estimates

\begin{tabular}{|c|c|c|c|c|c|c|c|c|}
\hline \multirow[b]{2}{*}{ Parameter } & \multirow{2}{*}{\multicolumn{2}{|c|}{$\mathrm{DF}$}} & \multirow[b]{2}{*}{ Estimate } & \multirow{2}{*}{$\begin{array}{l}\text { Standard } \\
\text { Error }\end{array}$} & \multirow{2}{*}{$\begin{array}{c}\text { Wald } \\
\text { Chi-square }\end{array}$} & \multirow{2}{*}{$\begin{array}{c}\text { Pr > } \\
\text { Chi-square }\end{array}$} & \multicolumn{2}{|c|}{ Standardized } \\
\hline & & & & & & & Estimate & $\exp$ (Est) \\
\hline Intercept & & 1 & 16.1756 & 2.5574 & 40.00 & $<.0001$ & & 999.000 \\
\hline Mean & & 1 & -0.0227 & 0.00172 & 173.38 & $<.0001$ & -0.748077 & 0.978 \\
\hline REMratio & & 1 & 31.0857 & 2.1521 & 208.64 & $<.0001$ & 1.923851 & 999.000 \\
\hline REMtime & & 1 & -0.00093 & 0.000079 & 139.71 & $<.0001$ & -1.581973 & 0.999 \\
\hline $\operatorname{ssc} 1$ & 0 & 1 & 0.6100 & 0.0981 & 38.64 & $<.0001$ & . & 1.840 \\
\hline $\operatorname{ssc} 2$ & 0 & 1 & 0.2155 & 0.0740 & 8.47 & 0.0036 & . & 1.240 \\
\hline SDNN_ & & 1 & -0.0119 & 0.00884 & 1.83 & 0.1764 & -0.089465 & 0.988 \\
\hline RMSSD & & 1 & 0.2690 & 0.0388 & 47.99 & $<.0001$ & 0.976693 & 1.309 \\
\hline Poincare & & 1 & -0.00313 & 0.00643 & 0.24 & 0.6265 & -0.026692 & 0.997 \\
\hline FD & & 1 & -11.9873 & 2.0720 & 33.47 & $<.0001$ & -0.452185 & 0.000 \\
\hline SDNNIDX & & 1 & 0.0190 & 0.00791 & 5.77 & 0.0163 & 0.099513 & 1.019 \\
\hline $\mathrm{HFW}$ & & 1 & $-2.42 E-6$ & $7.728 \mathrm{E}-7$ & 9.82 & 0.0017 & -0.280228 & 1.000 \\
\hline LFW & & 1 & $1.316 \mathrm{E}-7$ & $8.947 \mathrm{E}-8$ & 2.16 & 0.1413 & 0.059751 & 1.000 \\
\hline LFW_HFW & & 1 & 0.0226 & 0.00902 & 6.30 & 0.0121 & 0.070732 & 1.023 \\
\hline
\end{tabular}

Odds Ratio Estimates

$\begin{array}{lrr}\text { Input } & & \text { Odds Ratio } \\ & & 0.978 \\ \text { Mean } & & 999000 \\ \text { REMratio } & & 0.999 \\ \text { REMtime } & & 3.387 \\ \text { SSC1 } & 0 \text { VS } 1 & 1.539 \\ \text { SSC2 } & 0 \text { VS } 1 & 0.988 \\ \text { SDNN_ } & & 1.309\end{array}$




$\begin{array}{ll}\text { Poincare } & 0.997 \\ \text { FD } & 0.000 \\ \text { SDNNIDX } & 1.019 \\ \text { HFW } & 1.000 \\ \text { LFW } & 1.000 \\ \text { LFW_HFW } & 1.023\end{array}$

Step 3. Variable Poincare is removed:

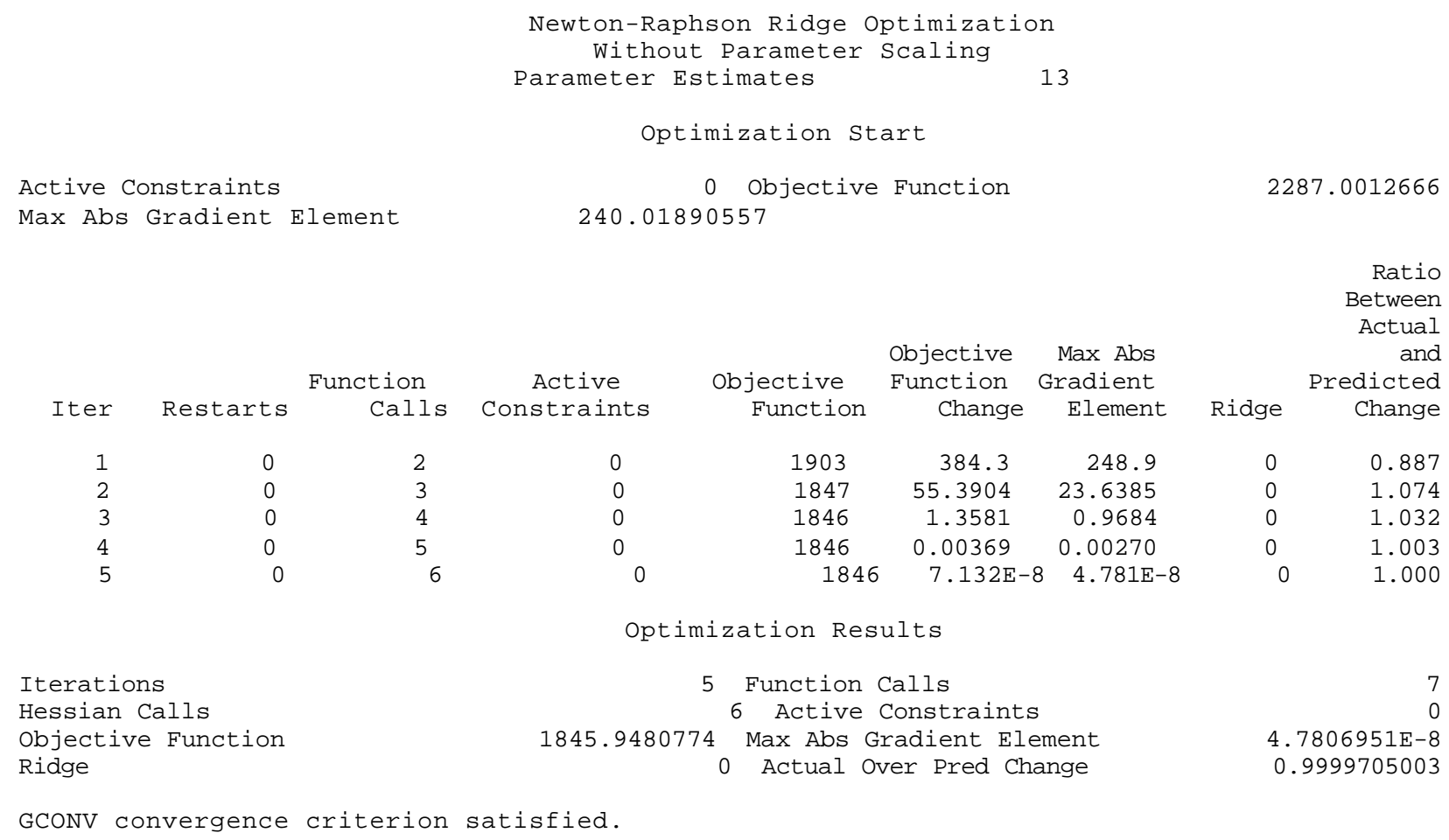

Model Fitting Information and Testing Global Null Hypothesis BETA=0

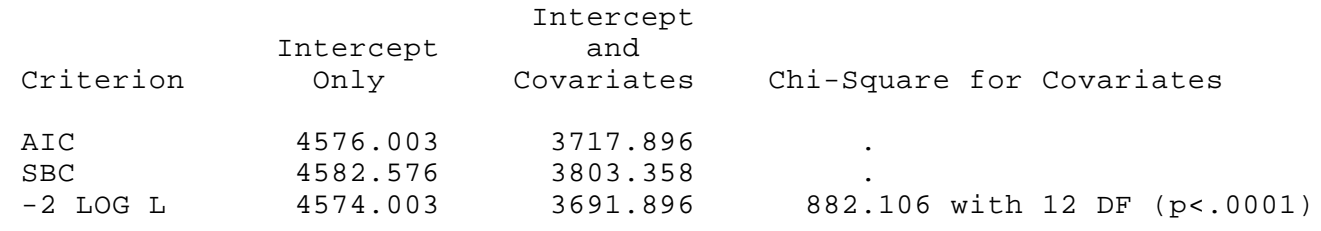

Type III Analysis of Effects

$\begin{array}{lcrr}\text { Effect } & \text { DF } & \begin{array}{c}\text { Wald } \\ \text { Chi-Square }\end{array} & \begin{array}{c}\text { Pr }> \\ \text { Chi-Square }\end{array} \\ \text { Mean } & 1 & 180.9696 & <.0001 \\ \text { REMratio } & 1 & 210.0247 & <.0001 \\ \text { REMtime } & 1 & 141.0249 & <.0001 \\ \text { SSC1 } & 1 & 39.2274 & <.0001 \\ \text { SSC2 } & 1 & 8.3864 & 0.0038 \\ \text { SDNN_ } & 1 & 6.2881 & 0.0122 \\ \text { RMSSD } & 1 & 50.7506 & <.0001 \\ \text { FD } & 1 & 35.7575 & <.0001 \\ \text { SDNNIDX } & 1 & 5.6308 & 0.0176 \\ \text { HFW } & 1 & 10.3362 & 0.0013 \\ \text { LFW } & 1 & 2.5643 & 0.1093 \\ \text { LFW_HFW } & 1 & 6.5256 & 0.0106\end{array}$


Analysis of Maximum Likelihood Estimates

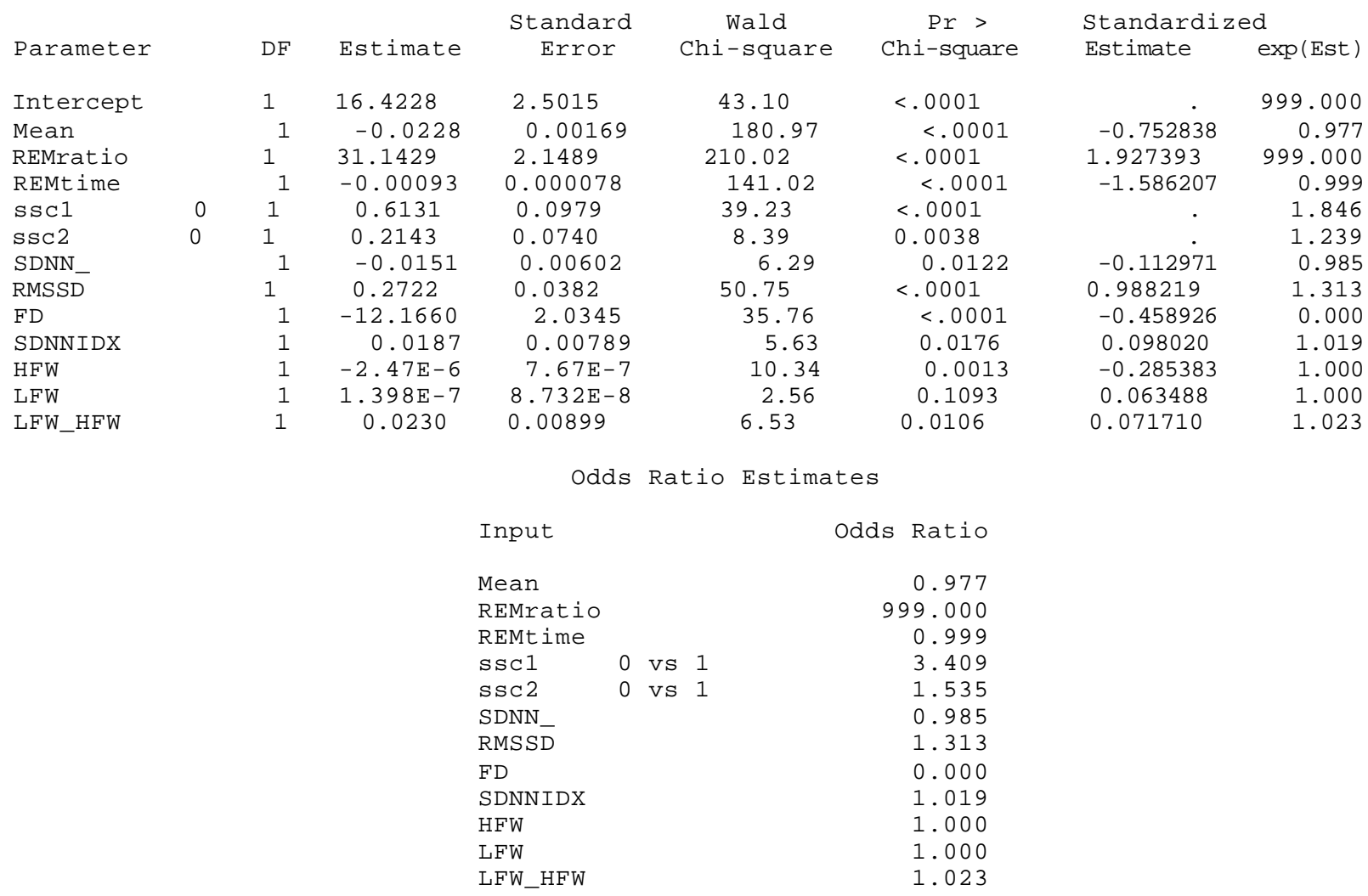

step 4. Variable LFW is removed:

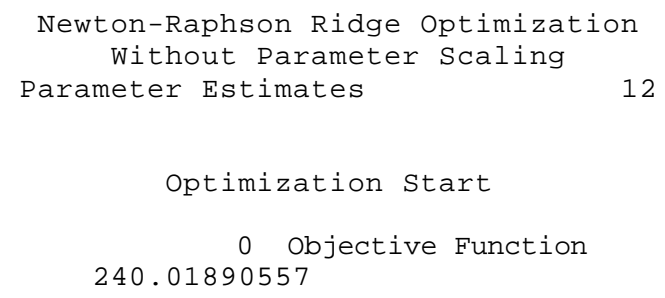

Ratio

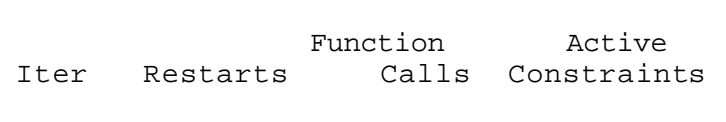
Objective Function

Objective Max Abs

Function Gradient Change Element

$\begin{array}{ccc}1904 & 383.4 & 248.6 \\ 1849 & 55.0404 & 23.4606 \\ 1847 & 1.2990 & 0.9308 \\ 1847 & 0.00299 & 0.00222 \\ 1847 & 2.861 \mathrm{E}-8 & 2.027 \mathrm{E}-8\end{array}$

0

0
0
0
0

2
3
4
5
6

Optimization Results

Iterations

Hessian Calls

objective Function

Ridge

GCONV convergence criterion satisfied.
5 Function Calls

6 Active Constraints

1847.2135742 Max Abs Gradient Element 0 Actual Over Pred Change Ratio
Between Actual and

$\begin{array}{cr}\text { Ratio } & \begin{array}{r}\text { Between } \\ \text { Actual } \\ \text { and }\end{array} \\ & \begin{array}{r}\text { Predicted } \\ \text { Change }\end{array} \\ & 0.886 \\ 0 & 1.073 \\ 0 & 1.031 \\ 0 & 1.002 \\ 0 & 1.000\end{array}$


Model Fitting Information and Testing Global Null Hypothesis BETA=0

\begin{tabular}{|c|c|c|c|c|}
\hline Critorion & Intercept & $\begin{array}{c}\text { Intercept } \\
\text { and }\end{array}$ & & covariatos \\
\hline criterion & 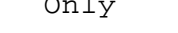 & & Chi-Square & covariates \\
\hline AIC & 4576.003 & 3718.427 & • & \\
\hline $\mathrm{SBC}$ & 4582.576 & 3797.315 & . & \\
\hline-2 LOG L & 4574.003 & 3694.427 & 879.575 & $(\mathrm{p}<.0001)$ \\
\hline
\end{tabular}

Type III Analysis of Effects

$\begin{array}{lrrr}\text { Effect } & \text { DF } & \begin{array}{c}\text { Wald } \\ \text { Chi-Square }\end{array} & \begin{array}{c}\text { Pr }> \\ \text { Chi-Square }\end{array} \\ \text { Mean } & 1 & 182.2547 & <.0001 \\ \text { REMratio } & 1 & 212.2842 & <.0001 \\ \text { REMtime } & 1 & 143.2830 & <.0001 \\ \text { SSC1 } & 1 & 42.9749 & <.0001 \\ \text { SSC2 } & 1 & 8.8760 & 0.0029 \\ \text { SDNN_ } & 1 & 3.9227 & 0.0476 \\ \text { RMSSD } & 1 & 52.3737 & <.0001 \\ \text { FD } & 1 & 37.6317 & <.0001 \\ \text { SDNNIDX } & 1 & 6.3890 & 0.0115 \\ \text { HFW } & 1 & 9.5677 & 0.0020 \\ \text { LFW_HFW } & 1 & 10.5128 & 0.0012\end{array}$

Analysis of Maximum Likelihood Estimates

\begin{tabular}{|c|c|c|c|c|c|c|c|c|}
\hline & & & & Standard & Wald & $\operatorname{Pr}>$ & \multicolumn{2}{|c|}{ Standardized } \\
\hline Parameter & & $\mathrm{DF}$ & Estimate & Error & Chi-square & Chi-square & Estimate & $\exp ($ Est) \\
\hline Intercept & & 1 & 16.6049 & 2.4977 & 44.20 & $<.0001$ & . & 999.000 \\
\hline Mean & & 1 & -0.0229 & 0.00169 & 182.25 & $<.0001$ & -0.755451 & 0.977 \\
\hline REMratio & & 1 & 31.2947 & 2.1479 & 212.28 & $<.0001$ & 1.936786 & 999.000 \\
\hline REMt ime & & 1 & -0.00094 & 0.000078 & 143.28 & $<.0001$ & -1.597156 & 0.999 \\
\hline ssc1 & 0 & 1 & 0.6362 & 0.0971 & 42.97 & $<.0001$ & . & 1.889 \\
\hline $\operatorname{ssc} 2$ & 0 & 1 & 0.2202 & 0.0739 & 8.88 & 0.0029 & • & 1.246 \\
\hline $\mathrm{SDNN}_{-}$ & & 1 & -0.0100 & 0.00506 & 3.92 & 0.0476 & -0.075011 & 0.990 \\
\hline RMSSD & & 1 & 0.2749 & 0.0380 & 52.37 & $<.0001$ & 0.998246 & 1.316 \\
\hline FD & & 1 & -12.4278 & 2.0259 & 37.63 & $<.0001$ & -0.468799 & 0.000 \\
\hline SDNNIDX & & 1 & 0.0199 & 0.00787 & 6.39 & 0.0115 & 0.104183 & 1.020 \\
\hline $\mathrm{HFW}$ & & 1 & $-2.28 E-6$ & $7.367 E-7$ & 9.57 & 0.0020 & -0.263718 & 1.000 \\
\hline LFW_HFW & & 1 & 0.0274 & 0.00846 & 10.51 & 0.0012 & 0.085676 & 1.028 \\
\hline
\end{tabular}

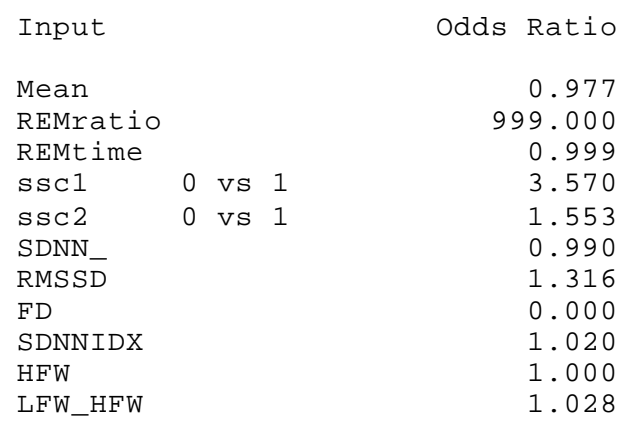

NOTE: No (additional) variables met the 0.05 significance level for removal from the model. 


\section{Summary of Backward Elimination Procedure}

\begin{tabular}{|c|c|c|c|c|c|}
\hline & Effect & & Number & Wald & $\operatorname{Pr}>$ \\
\hline Step & Removed & $\mathrm{DF}$ & In & Chi-Square & Chi-Square \\
\hline 1 & SDANN & 1 & 14 & 0.0309 & 0.8605 \\
\hline 2 & ApEn & 1 & 13 & 0.0402 & 0.8411 \\
\hline 3 & Poincare & 1 & 12 & 0.2369 & 0.6265 \\
\hline 4 & LFW & 1 & 11 & 2.5643 & 0.1093 \\
\hline
\end{tabular}

The selected model, based on the CHOOSE=XMISC criterion, is the model trained in Step 4 . It consists of the following effects:

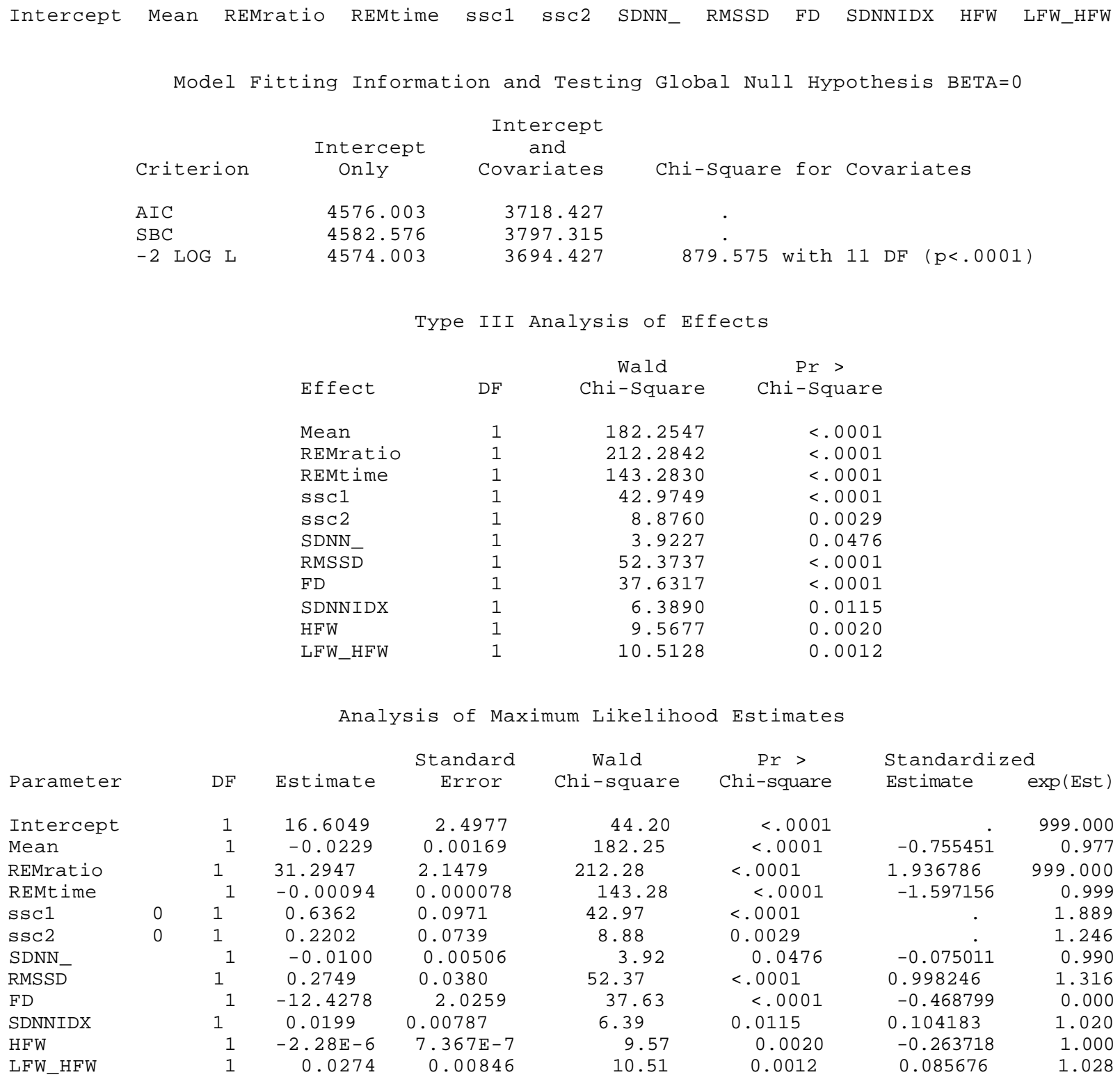


Odds Ratio Estimates

$\begin{array}{lllll}\text { Input } & & & \\ \text { Mean } & & & & \\ \text { REMratio } & & & \\ \text { REMtime } & & & & \\ \text { SSC1 } & 0 & \text { VS } & 1 \\ \text { SSC2 } & 0 & \text { VS } & 1 \\ \text { SDNN_- } & & & \\ \text { RMSSD } & & & \\ \text { FD } & & & & \\ \text { SDNNIDX } & & & \\ \text { HEW } & & & \\ \text { LEW_HFW } & & & \end{array}$

Odds Ratio

0.977

999.000

0.999

3.570

1.553

0.990

1.316

0.000

1. 020

1.000

1.028

\section{The FREQ Procedure}

Table of F_groupid by I_groupid

F_groupid(From: groupid)

I_groupid(Into: groupid)

\begin{tabular}{|c|c|c|c|c|}
\hline $\begin{array}{l}\text { Frequer } \\
\text { Percent } \\
\text { Row Pct } \\
\text { Col Pct }\end{array}$ & 0 & 1 & & Total \\
\hline 0 & $\begin{array}{r}43 \\
81 . \\
96 . \\
85 .\end{array}$ & $\begin{array}{r}2 \\
3 \\
56\end{array}$ & $\begin{array}{l}54 \\
91 \\
45 \\
62\end{array}$ & $\begin{array}{l}4469 \\
84.45\end{array}$ \\
\hline 1 & $\begin{array}{r}7 \\
13 . \\
85 . \\
14 .\end{array}$ & $\begin{array}{r}2 \\
14 \\
43\end{array}$ & $\cdot \begin{array}{l}18 \\
23 \\
34 \\
38\end{array}$ & $\begin{array}{r}823 \\
15.55\end{array}$ \\
\hline Total & $\begin{array}{r}50 \\
94 .\end{array}$ & $\begin{array}{l}80 \\
86\end{array}$ & $\begin{array}{l}72 \\
14\end{array}$ & $\begin{array}{r}5292 \\
100.00\end{array}$ \\
\hline
\end{tabular}




\section{BIBLIOGRAPHY}

[ADE99] Adelmann, H.G., Design of a PC-Based System for Time-Domain and Spectral Analysis of Heart Rate Variability. Computers and Biomedical Research, 32:77$92,1999$.

[ALD96] Aldroubi, A. and Unser, M., Wavelets in Medicine and Biology, CRC Press, Inc., 1996

[ANT90] Antila, K.J., Valimaki, I.A.T., Makela, M., Tuominen, J., Wilson, A.J., and Southall, D.P., Heart Rate Variability in Infants Subsequently Suffering Sudden Infant Death Syndrome (SIDS). Early Human Development, 22: 57-72, 1990.

[BAK77] Baker, T.L., and McGinty, D.J., Reversal of Cardiopulmonary Failure during Active Sleep in Hypoxic Kittens: Implications for Sudden Infant Death. Science 1977, Oct. 28; 198(4315): 419-421.

[BAR94] Barschdorff, D., Jaeger, A, and Trowitzsch, E., Automatic Assessment of an "Apnoea-Severity-Factor" combined with Heart Rate Analysis during Polysomnographic Examinations in Infant. Computers in Cardiology, 1994.

[BAK95] Bakardjian, H., and Yamamoto, K., Dynamic Non-Deterministic Characterization of HRV through Multiresolution Wavelet Decomposition. Annual International Conference of the IEEE Engineering in Medicine and Biology - Proceedings 17(2): 1063 - 1064, 1995.

[BER70] Bergman, A.B., Beckwith, J.B., Ray, G.C., eds., Sudden Infant Death Syndrome. Proceedings of the Second International Conference on the Causes of Sudden Infant Death in Infants. Seattle: Univ. of Washington Press, 1970.

[BER72] Bergman, A.B., Ray, G.C., Pomeroy, M.A., et al., Studies of the Sudden Infant Death Syndrome in King County, Washington. III. Epidemiol. Pediatr. 49:860-870, 1972.

[BER90] Berntson, G.G., Quigley, K.S., Jang, J.F., and Boysen, S.T., An Approach to Artifact Identification: Application to Heart Period Data. Psychophysiology, 27:586-598, 1990.

[BER97] Berntson, G.G., Bigger, T., Eckberg, D.L. et al., Heart Rate Variability: Origins, Methods, and Interpretive Caveats. Psychophysiology, 34:623-648, 1997.

[BER98] Berntson, G.G. and Stowell J.R., ECG Artifacts and Heart Period Variability: Don't Miss a Beat! Psychophysiology, 35:127-132, 1998. 
[BER00] Berry, M.J.A., and Linoff, G., Mastering Data Mining - the Art and Science of Customer Relationship Management. Wiley Computer publishing, Hohn Wiley \& Sons, Inc., 2000.

[BIG89] Bigger, J.T., Albrecht,P., Steinman, R.C., Rolnitzky, L.M., Fleiss J.L., and Cohen, R.J., Comparison of Time- and Frequency Domain-Based Measures of Cardiac Parasympathetic Activity in Holter Recordings After Myocardial Infarction. American Journal of Cardiology, 64: 536-538, 1989.

[BIG92] Bigger, J.T., Fleiss, J.L., Steinman, R.C., et al., Correlations Among Time and Frequency Domain Measures of Heart Period Variability Two Weeks After Acute Myocardial Infarction. The American Journal of Cardiology, 69: 891-898, 1992.

[BIG95] Bigger, J.T., Spectral Analysis of R-R Variability to Evaluate Autonomic Physiology and Pharmacology and to Predict Cardiovascular Outcomes in Humans. PartXI Diagnostic Evaluation.

[BOO78] Boor, C.de, A Practical Guide to Splines. Springer-Verlag, 1978.

[BO094] Bootsma, M., Swenne, C.A., et al., Heart Rate and Heart Rate Variability as Indexes of Sympathovagal Balance. The American Physiological Society, 1994.

[BRE84] Breiman, L., Friedman, J.H., Olshen, R.A., and Stone, C.J., Classification and Regression Trees. Wadsworth International Group, 1984.

[CAO99] Cao, C., Kohane, I.S., and McIntosh, N., Artifact Detection in Cardiovascular Time Series Monitoring Data from Preterm Infants. Proceedings / AMIA'99 Annual Symposium, pp. 207-211, 1999.

[CHE81] Cheung, M.N., Detection of and Recovery from Errors in Cardiac Interbeat Intervals, Psychophysiology, 18:341-346, 1981.

[COR96] Cornwell, A.C., Kim, A., and Feigenbaum, P., REM and NREM Sleep Duration Differs as a Function of Nighttime Interval in Infants at High Risk for SIDS. Annual International Conference of the IEEE Engineering in Medicine and Biology Proceedings No.1, Oct 31-Nov 3, pp.73-74, 1996.

[COR98] Corwin MJ, Lister G, Silvestri JM, Peucker M, Brooks LJ, Ward SL, Hunt CE, Neuman MR, Crowell DH, Colton T., Agreement among raters in assessment of physiologic waveforms recorded by a cardiorespiratory monitor for home use. Collaborative Home Infant Monitoring Evaluation (CHIME) Study Group. Pediatr. Res., 44(5):682-90, 1998.

[COS94] Costa,O., Lago, P., et al., Heart Rate Variability in 24-Hour Holter Recordings. Comparative Study Between Short- and Long -term Time and Frequency-domain Analyses. Journal of Electrocardiology, 27:251-254,1994. 
[CRO97] Crowell DH, Brooks LJ, Colton T, Corwin MJ, Hoppenbrouwers TT, Hunt CE, Kapuniai LE, Lister G, Neuman MR, Peucker M, Ward SL, Weese-Mayer DE, Willinger M. Infant polysomnography: reliability. Collaborative Home Infant Monitoring Evaluation (CHIME) Steering Committee. Sleep, 20(7):553-60, 1997.

[DON84] Donchin, Y., Caton, D., and Porges, S.W., Spectral Analysis of Fetal Heart Rate in Sheep: The Occurrence of Respiratory Sinus Arrhythmia. Am. J. Obstet. Gynecol. 148:1130-1135, 1984.

[EDL94] Edlinger, G., Litscher, G., and Pfurtscheller, G., Analysis of Cardiorespiratory Signals - Methodology and Applications in Infants. Biomed. Technik, 39:274-278, 1994.

[FIR97] Firstman, R., and Talan, J., The Death of Innocents, Bantam Books, New York, 1997.

[FRA92] Frawley, W., Piatetsky-Shapiro, G. and Matheus, C., Knowledge Discovery in Databases: An Overview. AI Magazine, pp. 213-228, 1992.

[GRO93] Grogaard, J.B., Apnea Monitors. Acta Pediatric suppl., 389:111-113,1993.

[HAD80] Haddad, G.G., Epstein, R.A., Epstein, M.A.F., Leistner, H.L. and Mellins, R.B., the R-R Interval and R-R Variability in Normal Infants During Sleep. Pediatric Research, 14:809-811, 1980.

[HAD87] Haddad, G.G., Jeng, H.J., Lai, T.L., and Mellins, R.B., Determination of Sleep State in Infants Using Respiratory Variability. Pediatric Research, Vol.21, No.6, pp556$562,1987$.

[HAR76] Harper, R.M., Hoppenbrouwers, T., Sterman, M.B., McGinty, D.J., and Hodgman, J., Polygraphic Studies of Normal Infants during the First Six Months of Life. I. Heart Rate and Variability as a Function of State. Pediatric Research, 10: 945-951, 1976.

[HAR78] Harper, R.M., Leake, B., Hoppenbrouwers, T., Sterman, M.B., McGinty, D.J., and Hodgman, J., Polygraphic Studies of Normal Infants and Infants at Risk for the Sudden Infant Death Syndrome: Heart Rate and Heart Rate Variability as a Function of State, Pediatric Research, 12: 778-785, 1978.

[HAR98] Harper, R.M. and Bandler, R., Finding the Failure Mechanism in Sudden Infant Death Syndrome. Nature Medicine, Vol.4, No. 2, pp157-158, Feb., 1998.

[HAS95] Hassoun, M.H., Fundamentals of Artificial Neural Networks, the MIT press, 1995.

[HAY99] Haykin, S., Neural Networks, a Comprehensive Foundation. Prentice-Hall, Inc., 1999. 
[HOF88] Hoffman, H.J., Darmus, K., Hillman, L., Krongrad, E., Risk Factors for SIDS: Results of the National Institute of Child Health and Human Development SIDS Cooperative Epidemiological Study. In: Schwartz, P.J., Southall, D.P., ValdesDapena, M., eds., The Sudden Infant Death Syndrome: Cardiac and Respiratory Mechanisms and Interventions. Vol.533. Annals N.Y. Academy of Sciences. New York: New York Academy of Sciences, 1988:13-30.

[HOL97] Holmstrom, L., Koistinen, P., and Laaksonen J., Neural and Statistical Classifiers --Taxonomy and Two Case Studies. IEEE Transactions on Neural Networks, 8(1): 5 $15,1997$.

[HOP80] Hoppenbrouwers, T., Hodgman, J.E., McGinty, D., Harper, R.M., and Sterman, M.B., Sudden Infant Death Syndrome: Sleep Apnea and Respiration in Subsequent Siblings. Pediatrics, 66:205-214,1980.

[HOP96] Hoppenbrouwers, T., Neuman, M.R., Corwin, M.J., et al., Multivariable Cardiorespiratory Monitoring at Home: Collaborative Home Infant Monitoring Evaluation (CHIME). Annual International Conference of the IEEE Engineering in Medicine and Biology - Proceedings, Part 1, pp. 61-62, 1996.

[HUI90] Huikuri, H.V., Kessler, K.M., Terracall, E., et al., Reproducibility and Circadian Rhythm of Heart Rate Variability in Healthy Subjects. The American Journal of Cardiology, Vol 65, pp.391-393, 1990.

[HUI94] Huikuri, H.V., Niemela, M.J., Ojala, S., Rantala, A., Ikaheimo, M.J., and Airaksinen, K.E.J., Circadian Rhythms of Frequency Domain Measures of Heart Rate Variability in Healthy Subjects and Patients with Coronary Artery Disease. Circulation, Vol 90, No.1, pp.121-126, 1994.

[HUN99] Hunt C.E., Corwin M.J., Lister G., Weese-Mayer D.E., Neuman M.R., Tinsley L., Baird T.M., Keens T.G., Cabral H.J., Longitudinal assessment of hemoglobin oxygen saturation in healthy infants during the first 6 months of age. Collaborative Home Infant Monitoring Evaluation (CHIME) Study Group. J Pediatr., 135(5):580-6, 1999.

[IZA91] Izard, C.E., Simons, R.F., Haynes, O.M., Porges, S.W., and Cohen, B., Infant Cardiac Activity: Developmental Changes and Relations with Attachment. Developmental Psychology, Vol27, NO.3, pp 432-439, 1991.

[JAF94] Jaffe, R.S., and Fung, D.L., Constructing a Heart-Rate Variability Analysis System. Journal of Clinical Monitoring, 10:45-58, 1994.

[JOB01] Jobe, A.H., What Do Home Monitors Contribute to the SIDS Problem? JAMA, 285:2244-2245, 2001.

[KAG87] Kagan, J., Peznick, J.S., and Snidman, N., The Physiology and Psychology of Behavioral Inhibition in Children. Child Development, 58:1459-1473,1087. 
[KAT88] Katz, M.J., Fractals and the Analysis of Waveforms. Computers in Biology and Medicine, 18:145-156, 1988.

\{KEJ86] Kejariwal, M.L., Brooks, E., Ojala, J., and Havlovick, B., Microprocessor-Based Home Monitor for Sudden Infant Death Syndrome. Proceedings of the Twelfth Annual Northeast Bioengineering Conference. 1986, pp 51-54.

[KEL79] Kelly, D.H., and Shannon, D.C., Periodic Breathing in Infants with Near-miss Sudden Infant Death Syndrome. Pediatrics, 63:255-360, 1979.

[KEL80] Kelly, D.H., Walker, A.M., Cahen, L., and Shannon, D.C., Periodic Breathing in Siblings of Sudden Infant Death Syndrome Victimes. Pediatrics, 66:515-520, 1980.

[KEL82] Kelly, D.H., Twanmoh, J., and Shannon, D.C., Incidence of Apnea in Siblings of Sudden Infant Death Syndrome Victims Studied at Home. Pediatrics, 70,128-131, 1982.

[KEL86] Kelly, D.H., Golub, H., Carley, D., and Shannon, D.C., Pneumograms in Infants Who Subsequently Died of Sudden Infant Death Syndrome. Journal of Pediatr., 109:249254, 1986.

[KEL91] Kelly, D.H., Pathak, A., and Meny, R., Sudden Severe Bradycardia in Infancy. Pediatr. Pulmonol, 10:199-204,1991.

[KLE87] Kleiger, R.E., Miller, J.P., Bigger, J.T., and Moss, A.J., Decreased Heart Rate Variability and Its Association with Increased Mortality After Acute Myocardial Infarction. American Journal of Cardiology, 59:256-262, 1987.

[KLE90] Kleiger, R.E., Miller, J.P., Krone, R.J., et al., The Independence of Cycle Length Variability and Exercise testing on Predicting Mortality of Patients Surviving Acute Myocardial Infarction. American Journal of Cardiology, 65:408-411, 1990.

[KLE91] Kleiger, R.E., Bigger, J.T., Bosner, M.S., et al, Stability Over Time of Variables Measuring Heart Rate Variability in Normal Subjects. American Journal of Cardiology, 68:626, 1991.

[KLE92] Kleiger, R.E., Stein, P.K., Bosner, M.S., and Rottman, J.N., Time Domain Measurements of Heart Rate Variability. Cardiology Clinics, Vol.10, No.3, pp.487498, 1992.

[KLE94] Kleinbaum, D.G., Logistic Regression: a Self-Learning Text. New York: Springer Verlag, 1994.

[KLU88] Kluge, K.A., Harper, R.M., Schechtman, V.L., Wilson, A.J., Hoffman, H.J., and Southall, D.P., Spectral Analysis Assessment of Respiratory Sinus Arrhythmia in 
Normal Infants and Infants Who Subsequently Died of Sudden Infant Death Syndrome. Pediatric Research, Vol.24, No.6, pp.677-682, 1988

[KOT91] Kothari, R., Klinkhachorn, P., and Nutter, R.S., An Accelerated Back Propagation Training Algorithm, Intl. Joint Conf. on Neural Network '91 Singapore, Nov. 18-21, 1991.

[KRA89] Kraus, J.F., Greenland, S., Bulterys, M., Risk Factors for Sudden Infant Death in the US Collaborative Perinatal Project. Int. J. Epidemiol. 18:113-120, 1989.

[KUL98] Kulkarni, S.R., Lugosi, G., and Venkatesh, S.S, Learning Pattern Classification --- A Survey. IEEE Transactions on Information Theory, 44(6): 2178 - 2201, 1998.

[KUL00] Kulp T.D., Corwin M.J., Brooks L.J., Peucker M., Fabrikant G., Crowell D.H., Hoppenbrouwers T., The effect of epoch length and smoothing on infant sleep and waking state architecture for term infants at 42 to 46 weeks postconceptional age. Sleep 23(7):893-9, 2000.

[LAN96] Landes, R.A., Scher, M.S., Sun, M., Sclabassi, R.J., Characterization of Heart Rate Dynamics in Infants as a Probe for Neural State and Age. $18^{\text {th }}$ Annual International Conference of the IEEE Engineering in Medicine and Biology Society, pp.16621663, 1996.

[LI97] Li, C., and Shusterman, V., et al., Wavelet Decomposition of Nonstationary Heart Rate Variability, Proceedings $-19^{\text {th }}$ International Conference - IEEE/EMBS, pp.8588, 1997.

[LIA96] Liao, D., Barnes, R.W., Chambless, L.E., and Heiss,G., A Computer Algorithm to Impute Interrupted Heart Rate Data for the Spectral Analysis of Heart Rate Variability--- the ARIC Study. Computers and Biomedical research, Vol. 29, pp.140$151,1996$.

[LEI80] Leistner, H.L., Haddad, G.G., Epstein, R.A., Lai, T.L., Epstein, M.F., and Mellins, R.B., Heart Rate and Heart Rate Variability during Sleep in Aborted Sudden Infant Death Syndrome. The Journal of Pediatrics, Vol. 97, No. 1, pp.51-55, 1980.

[LEV90] Berne, R.M., and Levy, M.N., Principles of Physiology. The C.V. Mosby Company, 1990.

[LIT95] Litvack, D.A., Oberlander, T.F., Carney, L.H., and Saul, J.P., Time and Frequency Domain Methods for Heart Rate Variability Analysis: A Methodological Comparison. Psychophysiology, 32:492-504,1995.

[MAL89] Malik, M., Cripps, T., Farrell, T. and Camm, A.J., Prognostic Value of Heart Rate Variability after Myocardial Infarction. A Comparison of Different Data-Processing Methods. Med. \& Biol. Eng. \& Comput., 27:603-611, 1989. 
[MAL892] Mallat, S.G., A Theory for Multiresolution Signal Decomposition: The Wavelet Representation. IEEE transactions, Pattn. Anal. Mach. Intell., 11(7):674 - 693, 1989.

[MAL95] Malik, M. and Camm, A.J., Heart Rate Variability. Futura Publishing Company, Inc., 1995.

[MAT92] Matthews, T.G., The Autonomic Nervous System - a Role in Sudden Infant Death Syndrome. Archives of Disease in Childhood, 67:654-656, 1992.

[MAZ98] Mazursky, J.E., Birkett, C.L., et al., Development of Baroreflex Influences on Heart Rate Variability in Preterm Infants. Early Human Development, 53(1): 37-52, 1998.

[MED97] Medigue, C., Bestel., J., Renard, S., et al., Discrete Wavelet Transform Applied to Heart Rate Variability Analysis in Iron-Deficient Anemic Infant. Proceedings of $19^{\text {th }}$ Inthernational Conference on IEEE/EMBS, pp 1613-1616, 1997.

[MEN94] Meny, R.G., Carroll, J.L., Carbone, M.T., Kelly, D.H., Cardiorespiratory Recordings from Infants Dying Suddenly and Unexpectedly at Home. Pediatrics, 93:44-49, 1994.

[MRO96] Mrowka, R., Schluter, B., Gerhardt, D., and Patzak, A., Heart Rate Control in Infants at High Risk for Sudden Infant Death Syndrome (SIDS). IEEE 1996.

[MR097] Mrowka, R., Schluter, B., Patzak, A, and Persson, P.B., Symbolic Dynamics Approach in the Analysis of Heart Rate in Premature Babies at High Risk for Sudden Infant Death Syndrome (SIDS). IEEE 1997.

[MYE86] Myers, G.A., Martin, G.J., Magid, N.M., Barnett, P.S., Schaad, J.W., Weiss, J.S., Lesch, M., and Singer, D.H., Power Spectral Analysis of Heart Rate Variability in Sudden Cardiac Death: Comparison to Other Methods. IEEE Transactions on Biomedical Engineering, Vol. BME-33, No. 12, 1986.

[NAE76] Naeye, R.L., Landis, B., Drage, J.S., Sudden Infant Death Syndrome: A Prospective Study. Am. J. Dis. Child. 130:1207-1210, 1976.

[NEU01] Neuman, M.R., Watson H, et a., Cardiopulmonary Monitoring at Home: the CHIME Monitor. Physiol. Meas., 22(2):267-286, 2001.

[NOC99] Nocedal, J. and Wright, S.J., Numerical Optimization. pp. 251-264, Springer-Verlag, New York, 1999.

[ORI92] Ori, Z., Monir, G., Weiss, J., Sayhouni, X. and Singer, D.H., Heart Rate Variability Frequency Domain Analysis. Cardiology Clinics, Vol.10, No.3, pp.499-533, 1992 
[PET80] Peterson, D.R., Chinn, N.M., Fisher, L.D. The Sudden Infant Death Syndrome: Repetitions in Families. J. Pediatr. 97:265-267, 1980

[PET86] Peterson, D.R., Sabotta, E.E., Daling,J.R., Infant Mortality Among Subsequent Siblings of Infants who Died of Sudden Infant Death Syndrome and Normal Infants. J. Pediatr. 108:911-914, 1986.

[PIC99] Pichot, V., Gaspoz, J.M., et al., Wavelet Transform to Quantify Heart Rate Variability and to Assess its Instantaneous Changes. J. Appl. Physiol. 86(3):10811091, 1999.

[PIK99] Pikkujamsa, S.M., Makikallio, T.H., Sourander, L.B., et al., Cardiac Interbeat Interval Dynamics from Childhood to Senescence. Comparison of Conventional and New Measures Based on Fractals and Chaos Theory. Circulation, July 27, 1999.

[PIN93] Pincus, S.M., Cummins, T.R., and Haddad, G.G., Heart Rate Control in Normal and Aborted-SIDS infants, 1993.

[PIN94] Pincus, S.M., Goldberger, A.L., Physiological Time-Series Analysis: What Does Regularity Quantify? Modeling in Physiology. The American Physiological Society, 1994.

[PIN95] Pincus, S.M., Quantifying Complexity and Regularity of Neurobiological Systems. Methods in Neurosciences, Vol.28, 1995.

[POE94] Poets, C.F., Southall, D.P., Recent Developments in Research into Sudden Infant Death. Thorax 49:196-197, 1994.

[POR92] Porges, S.W., and Byrne, E.A., Research methods for measurement of heart rate and respiration. Biological Psychology 34:93-130, 1992.

[POT98] Potts, W. J.E., Data Mining Primer: Overview of Applications and Methods. SAS Institute Inc., 1998.

[PRE92] Press, W.H., Teukolsky, S.A. et al., Numerical Recipes in C, The Art of Scientific Computing. Cambridge University Press, 1992.

[RAM01] Ramanathan, R., Corwin, M.J., et al., Cardiorespiratory Events Recorded on Home Monitors, Comparison of Healthy Infants With Those at Increased Risk for SIDS. JAMA, 285:2199-2207, 2001.

[ROS34] Rosenblueth, A., and Simeone, F.A., The Interrelations of Vagal and Accelerator Effects on the Accelerator Effects on the Cardiac Rate. American Journal of Physiology, 110:42-45, 1934. 
[ROS99] Rosenstock, E.G., Cassuto, Y., and Zmora, E., Heart Rate Variability in the Neonate and Infant: Analytical Methods, Physiological and Clinical Observations. Acta Pediatr., 88:477-482, 1999.

[ROT90] Rottman, J.N., Steinman, R.C., Albrecht, P. et al., Efficient Estimation of the Heart Period Power Spectrum Suitable for Physiologic or Pharmacologic Studies. The American Journal of Cardiology, 66:1522-1523, 1990.

[SAP91] Sapoznikov, D., Luria, M.H., Mahler, Y., Gotsman, M.S., Methods of Arrhythmia and Artifact Removal in Heart Rate Variability Analysis. Annual International Conference of the IEEE Engineering in Medicine and Biology Society. Vol. 13, No.2, 1991.

[SAU88] Saul, J.P., Arai, Y., Berger, R.D. et al., Assessment of Autonomic Regulation in Chronic Congestive Heart Failure by Heart Rate Spectral Analysis. American Journal of Cardiology, 61:1292-1299, 1988.

[SAU90] Saul, J.P., Rea, R.F. et. al., Heart Rate and Muscle Sympathetic Nerve Variability during Reflex Changes of Autonomic Activity. American Journal of Psychology, 26:582-588, 1990.

[SCH89] Schechtman, V.L., Harper, R.M., Kluge, K.A., Wilson, A.J., Hoffman, H.J., and Southall, D.P., Heart Rate Variation in Normal Infants and Victims of the Sudden Infant Death Syndrome. Early Human Development, 19:167-181,1989.

[SCH91] Schechtman, V.L., Harper, R.M., Wilson, A.J., and Southall, D.P., Sleep Apnea in Infants Who Succumb to the Sudden Infant Death Syndrome. Pediatrics, 87:841-846, 1991.

[SCH92] Schechtman, V.L., Raetz, S.L., Harper, R.K., Garfinkel, A., Wilson, A.J., Southall, D.P., and Harper, R.M., Dynamic Analysis of Cardiac R-R Intervals in Normal Infants and in Infants Who Subsequently Succumbed to the Sudden Infant Death Syndrome. Pediatric Research, 31:606-612, 1992.

[SCH98] Schechtman, V.L., Henslee, J.A., and Harper, R.M., Developmental Patterns of Heart Rate Variabiliyt in Infants with Persistent Apnea of Infancy. Early Human Development, 50:251-262,1998.

[SHE94] Sherwood, L., Fundamentals of Physiology. A Human Perspective, 1994.

[SHI99] Shimazu, M., Ojima, S. and Takasugi, S.I. et al., Time-Frequency Analysis of Heart Rate Variability Using Complex Discrete Wavelet Transform. Annual International Conference of the IEEE Engineering in Medicine and Biology - Proceedings, pp. 958, 1999. 
[SOU87] Southall, D.P., Alexander, J.R., Stebbens, V.A., Taylor, V.G., and Janczynski, R.E., Cardiorespiratory patterns in Siblings of Babies with Sudden Infant Death Syndrome. Arch. Dis. Child, 62:721-726,1987.

[SPI87] Spicer, C.C. and Lawrence, C.J., Statistical Analysis of Heart Rates in Subsequent Victims of Sudden Infant Dearth Syndrome. Statistics in Medicine, 6:159-166, 1987.

[SPI93] Spiers, J.P., Silke, B., McDermott, U., et al., Time and Frequency Domain Assessment of Heart Rate Variability: a Theoretical and Clinical Appreciation. Clinical Autonomic Research, 3:145-158, 1993.

[STE72] Steinschneider, A., Prolonged Apnea and the Sudden Infant Death Syndrome Clinical and Laboratory Observations. Pediatrics, 50:646-654, 1972.

[STE88] Stevens, V.G., Wilson, A.J., Franks, C.I., and Southall, D.P., Techniques for the Analysis of Long-Term Cardiorespiratory Recordings from Infants. Med. \& Biol. Eng. \& Computing, 26:282-288,1988.

[TASK96] Task Force of the European Society of Cardiology and the North American Society of Pacing and Electrophysiology, Heart Rate Variability, Standards of Measurement, Physiological Interpretation and Clinical Use, 1996.

[VAL88] Valimaki, I.A., Nieminen, T., Antila, L.J., and Southall, D.P., Heart-Rate Variability and SIDS. Examination of Heart-Rate Patterns Using an Expert System Generator. Annals New York Academy of Sciences, 533:228-237, 1988.

[WEE00] Weese-Mayer D.E., Corwin M.J., Peucker M.R., Di Fiore J.M., Hufford D.R., Tinsley L.R., Neuman M.R., Martin R.J., Brooks L.J., Davidson Ward S.L., Lister G., Willinger M., Comparison of apnea identified by respiratory inductance plethysmography with that detected by end-tidal $\mathrm{CO}(2)$ or thermistor. The CHIME Study Group. Am J Respir Crit Care Med, 162(2 Pt 1):471-80, 2000.

[VER93] Vertes, R.P., Perry, G.W., Sudden Infants Death Syndrome: A Theory. Neuroscience and Biobehavioral Reviews, 17:305-312,1993.

[WEG88] Wegman, M.E., Annual Review of Vital Statistics -1987. Pediatrics 82:817- 827, 1988.

[WIK97] Wiklund,U., Akay, M. and Niklansson, U., Short-Term Analysis of Heart_Rate Variability by Adapted Wavelet Transforms. IEEE Engineering in Medicine and Biology, pp.113-118, 1997.

[WIL82] Wilson, A.J. and Franks, C.I., The Sheffield respiration analysis system. IEEE Proc. A, 129:702-706, 1982.

[WIL89] Willinger, M. SIDS: A challenge. J. NIH Res. 1:73-80, 1989. 
[YAN97] Yang, F., Liao, W., Modeling and Decomposition of HRV Signals with Wavelet Transforms. IEEE Engineering in Medicine and Biology, pp.17-22, 1997.

[YER98] Yeragani, V.K., Sobolewski, E., Jampala, V.C., Kay, J., Yeragani, S., Igel, G., Fractal Dimension and Approximate Entropy of Heart Period and Heart Rate: Awake Versus Sleep Differences and Methodological Issues. Clinical Science, 95: 295-301, 1998.

[ZBI88] Zbilut, J.P., Murdock, D.K., Lawson, L., Lawless, C.E., Dreele, M.M.V., and Porges, S.W., Use of Power Spectral Analysis of Respiratory Sinus Arrhythmia to Detect Graft Rejection. Journal of Heart Transplant, 7:280-287, 1988. 